\title{
Determination of the Proton's Weak Charge via Parity Violating Electron Scattering
}

Joshua Russell Hoskins

Williamsburg, Virginia

Master of Science, College of William and Mary, 2007

Bachelor of Arts, Eastern Kentucky University, 2006

A Dissertation presented to the Graduate Faculty

of the College of William and Mary in Candidacy for the Degree of Doctor of Philosophy

Department of Physics

The College of William and Mary

August 2015 
(C) 2015

Joshua Russell Hoskins

All rights reserved. 


\title{
APPROVAL PAGE
}

This Dissertation is submitted in partial fulfillment of the requirements for the degree of

Doctor of Philosophy

Joshua Russell Hoskins

Approved by the Committee, June, 2015

Committee Chair
Professor David S. Armstrong, Physics
The College of William and Mary
CEBAF Professor Roger D. Carlini, Physics
The College of William and Mary

The College of William and Mary

\begin{abstract}
$\overline{\text { Assistant Professor Wouter Deconinck, Physics }}$
\end{abstract}
The College of William and Mary

Associate Professor Josh Erlich, Physics

The College of William and Mary

Staff Scientist Bradley Sawatzky, Physics

Thomas Jefferson National Accelerator Facility 


\begin{abstract}
The $Q_{\text {weak }}$ experiment, which completed running in May of 2012 at Jefferson Laboratory, has measured the parity-violating asymmetry in elastic electron-proton scattering at four-momentum transfer $Q^{2}=0.025(\mathrm{GeV} / c)^{2}$ in order to provide the first direct measurement of the proton's weak charge, $Q_{w}^{p}$. The Standard Model makes firm predictions for the weak charge; deviations from the predicted value would provide strong evidence of new physics beyond the Standard Model. Using an $89 \%$ polarized electron beam at $145 \mu \mathrm{A}$ scattering from a $34.4 \mathrm{~cm}$ long liquid hydrogen target, scattered electrons were detected using an array of eight fused-silica detectors placed symmetric about the beam axis. The parity-violating asymmetry was then measured by reversing the helicity of the incoming electrons and measuring the normalized difference in rate seen in the detectors. The low $Q^{2}$ enables a theoretically clean measurement; the higher order hadronic corrections are constrained using previous parity-violating electron scattering world data. The experimental method will be discussed, with recent results constituting $4 \%$ of our total data and projections of our proposed uncertainties on the full data set.
\end{abstract}




\section{TABLE OF CONTENTS}

Acknowledgments . . . . . . . . . . . . . . . . . . . . v v

Dedication ............................. vii

List of Tables . . . . . . . . . . . . . . . . . viii

List of Figures $\ldots \ldots \ldots \ldots \ldots \ldots \ldots \ldots \ldots \ldots \ldots \ldots$

CHAPTER

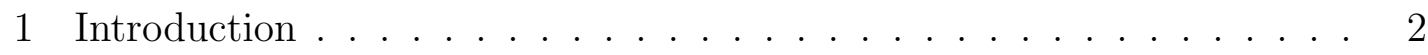

1.1 Fundamental Symmetries of the Standard Model . . . . . . . . . . 2

1.1.1 Fundamental Symmetries . . . . . . . . . . . . . . . 2

1.1.2 Standard Model Overview . . . . . . . . . . . . . 5

1.2 Electroweak Theory . . . . . . . . . . . . . . . . . . . . 9 9

1.2.1 Discrete Symmetry and Spin . . . . . . . . . . . . . . 9

1.2 .2 Electroweak Unification . . . . . . . . . . . . . . . . 11

2 The $\mathrm{Q}_{\text {weak }}$ Experiment . . . . . . . . . . . . . 16

2.1 Experimental Motivation . . . . . . . . . . . . . . . 16

2.2 Parity-Violating $e+p$ Scattering . . . . . . . . . . 18

2.2 .1 Neutral-Weak Interaction . . . . . . . . . . . . . . . 18

2.2.2 Low Momentum Electron Scattering . . . . . . . . . . 19

2.2 .3 Physics Asymmetry . . . . . . . . . . . . . . . . 23

2.2.4 Precision Determination of $\sin ^{2} \theta_{w} \ldots \ldots \ldots \ldots$

$2.2 .5 \gamma Z^{0}$ Box Diagram . . . . . . . . . . . . . . . 29 
3 Experimental Setup . . . . . . . . . . . . . . . . 31

3.1 Experimental Setup . . . . . . . . . . . . . . . . . 31

3.2 Polarized Electron Beam . . . . . . . . . . . . . . . . . . . . 33

3.2.1 Continuous Electron Beam Accelerator Facility . . . . . . . 33

3.2 .2 Polarized Source and Injector _ . . . . . . . . . . . 34

3.2 .3 Beam Position Monitors . . . . . . . . . . . . . . . 38

3.2 .4 Beam Current Monitors . . . . . . . . . . . . . . . . . 41

3.3 Electron Beam Polarimetry . . . . . . . . . . . . . . . . . 42

3.3.1 Møller Polarimeter . . . . . . . . . . . . . . . . . . . . 43

3.3.2 Compton Polarimeter . . . . . . . . . . . . . . . . . . 44

3.4 Primary $l \mathrm{H}_{2}$ Target . . . . . . . . . . . . . . . 46

3.5 Infrastructure $\ldots \ldots \ldots \ldots \ldots \ldots \ldots \ldots$

3.5.1 Collimation System . . . . . . . . . . . . . . . . 48

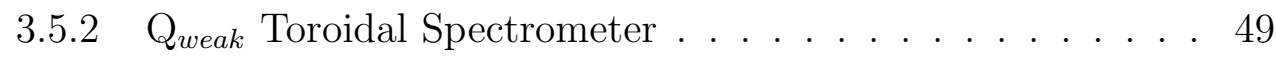

3.5 .3 Shielding Wall . . . . . . . . . . . . . . . . 50

3.5.4 Quartz Čerenkov Detectors . . . . . . . . . . . . . . . 50

3.5.5 Beamline Shielding . . . . . . . . . . . . . 53

3.5.6 Beam Modulation System _. . . . . . . . . . . . . 54

3.6 Tracking System . . . . . . . . . . . . . . . . . 55

3.6.1 Horizontal Drift Chambers . . . . . . . . . . . . . 56

3.6 .2 Vertical Drift Chambers . . . . . . . . . . . . . 57

3.6 .3 Trigger Scintillators . . . . . . . . . . . . . . . . . 61

3.7 Rotator . . . . . . . . . . . . . . . . 62

3.7 .1 Structural . . . . . . . . . . . . . . 63

3.7 .2 Linear Motion System _. . . . . . . . . . . . 65

3.7 .3 Rotational Motion System _. . . . . . . . . . . . 67 
3.7.4 Motion Controls System . . . . . . . . . . . . . . . . 69

3.7.5 Performance and Repeatability _. . . . . . . . . . 73

4 Beam Modulation . . . . . . . . . . . . . . . . . 75

4.1 Modulation System . . . . . . . . . . . . . . . 75

4.1.1 Beam Modulation and Helicity-Correlated Beam Systematics 75

4.1.2 Beam Modulation System Instrumentation . . . . . . . . 76

4.1 .3 Methodology . . . . . . . . . . . . . . . . . 82

4.1.4 Modulation Regression Analysis . . . . . . . . . . . . . 90

4.1.5 Residual Correlations . . . . . . . . . . . . . . . . . 120

4.1.6 Average Position Differences . . . . . . . . . . . . . . . 121

4.1.7 Asymmetry Correction . . . . . . . . . . . . . . . . 122

5 Systematic Uncertainties . . . . . . . . . . . . . . . . . . . 124

$5.1 \mathrm{Q}^{2}$ Systematics . . . . . . . . . . . . . . . . . 124

$5.1 .1 \quad$ Scattering Kinematics . . . . . . . . . . . . . . . 124

5.1 .2 Beam Energy . . . . . . . . . . . . . . . . . . 126

5.1 .3 Scattering Angle . . . . . . . . . . . . . . . . 127

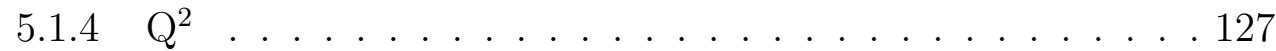

5.2 Backgrounds . . . . . . . . . . . . . . . . . . . . . . . . 128

5.2.1 Aluminium Target Window Background . . . . . . . . 129

5.2 .2 Aluminium Target Asymmetry . . . . . . . . . . . . . . . 129

5.2 .3 Aluminium Dilution . . . . . . . . . . . . . . . . . 131

5.2 .4 Beamline Background . . . . . . . . . . . . . . . . 133

5.2 .5 Beamline Background Dilution . . . . . . . . . . . . . 134

5.2 .6 Inelastic Background . . . . . . . . . . . . . . 136

5.2 .7 Total Neutral Background . . . . . . . . . . . . . 137 
5.2.8 Experimental Bias Corrections . . . . . . . . . . 139

5.3 Beam Polarization . . . . . . . . . . . . . . . . . . . . 140

5.3.1 Møller Polarimetry . . . . . . . . . . . . . . . . . 140

6 Results and Discussion . . . . . . . . . . . . . . . . . . . . 145

$6.1 e+p$ Asymmetry Results . . . . . . . . . . . . . . . . . 145

6.2 Conclusion \& Future Work . . . . . . . . . . . . . . . . . . . . 154

APPENDIX A

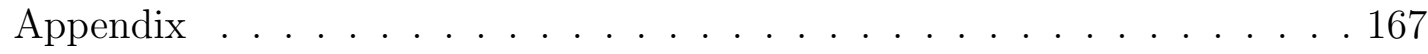

A.1 Detector Error Weighting . . . . . . . . . . . . 167

A.2 Rotator Study . . . . . . . . . . . . . . . . . 169

A.3 Background Correction Error Propagation . . . . . . . . . . . . . 179

A.4 Beam Modulation Improvements . . . . . . . . . . . . . . . . . . 180 


\section{ACKNOWLEDGMENTS}

The road to this point, completing my studies, writing this dissertation, has been a long one and I would be remiss to say that I have gotten here on my own merits; we truly are the sum of our parts. Throughout my studies, I have had supportive colleagues, friends, and family. First, I should thank my family. My interest in science and discovery was fostered early with my father teaching a fifth grader about chemistry, physics, and the occasional engineering project such as building my own stereo speaker from household materials or building a potato cannon. Though I wasn't always a focused student, I was lucky enough to have the encouragement and support that allowed me to find the interests that started the journey I am now finishing. Without the support received from my mother and father, getting here would not have been possible.

Throughout my undergraduate and graduate studies I was lucky enough meet and work with a number of wonderful people. From Eastern Kentucky University, where I did my undergraduate studies, I would like to thank Dr. Garett Yoder, Dr. Marco Ciocca, and Dr. Jerry Cook for their help and guidance. A special thanks goes to Dr. Christopher Kulp who I was lucky enough to work with during my undergraduate research, and who is the reason I am now graduating from the College of William and Mary. During my graduate career at William and Mary I was lucky enough to have two excellent scientific mentors; Dr. David Armstrong and the late Dr. Mike Finn. Without the latter I would not have gotten into the field of experimental particle physics. He was a mentor and friend who is dearly missed by many; myself included. I would also like to thank David Armstrong for taking up the mantle of advising me and seeing me through to the end. Without his insight and mentoring I could not have come this far; it was a pleasure and honor working with him.

With any scientific endeavour, there are often many people, working countless hours to do great things. The Qweak experiment, being no exception, was made possible by a large number of talented and hard working individuals both inside and out of academia. I would like to thank all of the Qweak collaboration for their hard work and dedication for making this measurement possible. Special mention includes Roger Carlini, Greg Smith, Paul King, Mark Pitt, Kent Paschke, Stephen Wood, Wouter Deconinck, and Jeong Han Lee. From Jefferson laboratory I would like to give special thanks to David Mack for his countless hours of discussion and mentoring; if knowledge imparted to graduate students was money you would be a 
rich man. I would also like to thank Brad Sawatzky for his insight, friendly discussions, debugging, and coffee. Lastly, I would like to thank Dave Gaskell for helping me throughout my time at the laboratory; without Dave I would have gotten nothing done in Hall C.

In addition to the scientist at Jefferson Lab, I would like to recognize staff and engineers that made the experiment not only a success but possible at all. These include Chris Cuevas and the Fast Electronics group, the Polarized Target group, Walter Kellner, Andy Kenyon, Paulo Medeiros, and all of the Hall C technical staff. Without your work none of the work presented in this dissertation would have been possible.

As with any experiment there is often an army of graduate students working behind the scenes to make things happen. I would like to thank you all for your hard work and dedication. Special mention goes to John Leacock, Katherine Myers, Amendra Narayan, Scott MacEwan, Josh Magee, Donald Jones, John Leckey, Nuruzzaman, Rakitha Beminiwattha, Adesh Subedi, and Buddini Waidyawansa. It is only through your work that we were able to finish this experiment. It was a pleasure and honor to work with you all.

Lastly, I must thank my wonderful wife Kerry. She has been an unyielding source of love, support, reassurance, coffee, and proofreading in my life over the last nine years. Whether it was small things around the house when I was busy at work, bouts of doubt and depression, or helping with the latest piece of mechanical design, I am truly lucky to have met someone like her. I am here because you were there. 
I dedicate this thesis to my parents and my wife Kerry. 


\section{LIST OF TABLES}

2.1 The vector and axial couplings interacting with $Z^{0}$ are shown for each flavor of lepton. . . . . . . . . . . . . . . . . . . 20

2.2 Recent calculations of $\square_{\gamma \mathrm{Z}}^{V}\left(E, Q^{2}\right)$ and its uncertainty at the kinematics of this measurement. . . . . . . . . . . . . . . . 30

3.1 Design Parameters for the $\mathrm{Q}_{\text {weak }}$ Experiment . . . . . . . . . . . . . . 32

3.2 Hall C BPMs used to construct virtual target BPMs. BPM 3H09b was removed due to functionality issues in the second half of running. 41

3.3 Beam Current Monitors activity periods. The monitors used to form the qwk_charge variable are listed. BCMs 1-2 were used primarily during Run I while BCMs 5-8 were being commissioned. BCMs 5-8 were built with lower noise digital receivers. . . . . . . . . . . . . . . 42

4.1 Example of a standard run configuration from Run I modulation. These numbers were adjusted throughout the experiment as the beam optics changed. . . . . . . . . . . . . . . . . . 81

4.2 List of phases used in the different modulation sets, in units of degrees. 91

4.3 Wien average sensitivities for each modulation set. Positions sensitivities are in units of $\mathrm{pm} / \mathrm{mm}$ and angle sensitivities are in units of

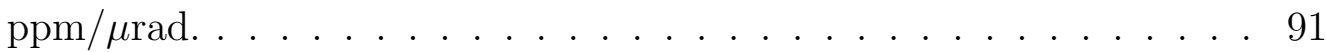

4.4 Wien level total corrections for each modulation Set. . . . . . . . . 110

4.5 Detector yield monopole in ppm before and after correction using Wien average modulation sensitivities. . . . . . . . . . . . . . 117

4.6 Detector yield dipole response in ppm before and after correction using Wien average modulation sensitivities. . . . . . . . . . . . . . . 118

4.7 Wien-level sensitivities for the main detector system. The Run I value represents the error weighted average of each Wien-level result. Positions are given in ppm/mm and angles are given in $\mathrm{ppm} / \mu \mathrm{rad} . \quad 118$

4.8 Wien-Average Position Difference for Run I after $6 \sigma$ cut. Positions are given in $\mathrm{nm}$ and angles are given in nrad. . . . . . . . . . . . . 120 
4.9 The residual correlations of the main detector to each degree of freedom in the beam after beam modulation corrections. Each corrections was determined and is listed as the Wien average value. Position correlations are given in $\mathrm{ppb} / \mathrm{nm}$ and angular correlations are given in ppb/nrad . . . . . . . . . . . . . . . . . . . 121

4.10 The number of runlets lost due to $\mathrm{n} \sigma$ cuts applied to the position difference data. . . . . . . . . . . . . . . . . . . . . 122

4.11 Run I modulation corrections to the measured asymmetries by Wien. 122

5.1 Systematic errors associated with the Møller measurement. . . . . . . 142

5.2 Individual beam polarization measurements during Run I. . . . . . . 143

6.1 The asymmetry and associated dilution for each background source. . 146

6.2 Breakdown of the systematic errors going into the final uncertainty. . 148

A.1 Angular and radial repeatability values measured after moving from HOME $\rightarrow 45^{\circ} \rightarrow$ HOME. Radial values are shown in millimeters and angular values are in milli-degrees. . . . . . . . . . . . . 170

A.2 Angular and radial repeatability values measured after moving from HOME $\rightarrow 45^{\circ} \rightarrow$ HOME. Radial values are shown in millimeters and angular values are in milli-degrees. . . . . . . . . . . . . 170

A.3 Radial repeatability after moving the chambers from the extended (in beam) location to the retracted (out of beam) and back. All values are given in millimeters. . . . . . . . . . . . . . . . . . 171

A.4 Radial repeatability after moving the chambers from the extended (in beam) location to the retracted (out of beam) and back. All values are given in millimeters. . . . . . . . . . . . . . . . 171

A.5 Shown is the extracted angle with respect to the home position. . . . 172

A.6 Unfortunately we did not get survey locations for all points on each chamber. This table is listed for completeness. . . . . . . . . . . 172

A.7 Shown is the extracted angle with respect to the home position. . . . 173

A.8 Shown is the extracted angle with respect to the home position. . . . 173

A.9 Shown is the extracted angle with respect to the home position. . . . 174

A.10 Shown is the extracted angle with respect to the home position. . . . 174

A.11 Shown is the extracted angle with respect to the home position. . . . 174 
A.12 Shown is the extracted angle with respect to the home position. . . . 175

A.13 The repeatability of the measured angle and radius of the chambers after repeated rotation to the $-90^{\circ}$ position. Angles are shown in milli-degress and radial values are shown in millimeters. . . . . . . . . 175

A.14 The repeatability of the measured angle and radius of the chambers after repeated rotation to the $-90^{\circ}$ position. Angles are shown in milli-degress and radial values are shown in millimeters. . . . . . . . . 175

A.15 The standard deviation of the radial measurement for each point in each position in theta. Extracted values are shown for the radius in both the $(\mathrm{x}, \mathrm{y})$ plane and the $(\mathrm{x}, \mathrm{y}, \mathrm{z})$ plane. Values in $\mathrm{mm}$. . . . . . . . 176

A.16 The standard deviation of the radial measurement for each point in each position in theta. Extracted values are shown for the radius in both the $(\mathrm{x}, \mathrm{y})$ plane and the $(\mathrm{x}, \mathrm{y}, \mathrm{z})$ plane. Values in $\mathrm{mm} \mathrm{.} \mathrm{.} \mathrm{.} \mathrm{.} \mathrm{.} \mathrm{.} 176$

A.17 The standard deviation of the angles measured with respect to home of each measurement shown in Tables A.5-A.11. . . . . . . . . . . . 177

A.18 The standard deviation of the angles measured with respect to home of each measurement shown in Tables A.6-A.12. . . . . . . . . . . . . 177

A.19 For each rotational position the distance between pairs of tooling ball locations (see Fig. A.1) is calculated. As these are static points the distance between them should not change. This gives a good estimate of the resolution with which the points were measured. . . . . . . . 178

A.20 For each rotational position the distance between pairs of tooling ball locations (see Fig. A.1) is calculated. As these are static points the distance between them should not change. This gives a good estimate of the resolution with which the points were measured. . . . . . . 178 


\section{LIST OF FIGURES}

1.1 The Standard Model of Particle Physics as presently determined. Fermions (1/2 integer spin) are divided into three generations with similar properties and increasing mass. Fermions are divided into leptons (do not interact via the strong coupling) and quarks. Force carriers are represented by the integer spin bosons to the right. Together these make up the Standard Model and have been extremely successful in explaining experimental data over the last 50 years[1].

2.1 Tree level diagrams for ep scattering in the case of the electromagnetic and neutral-weak interactions. . . . . . . . . . . . . . . . 20

2.2 The electromagnetic interaction at $\mathcal{O}(\alpha)$ (left) and $\mathcal{O}\left(\alpha^{2}\right)$ (right). The vacuum polarization screens the bare charge of the electromagnetic interaction at the vertex. . . . . . . . . . . . . . . . 26

2.3 The running of the weak mixing angle using the $\overline{M S}$ renormalization scheme[2]. The width of the curve represents the theoretical uncertainty in the calculation. The Z-pole value is given at $Q^{2}=M_{Z}$. . . 27

2.4 The one loop contribution to $Q_{w}^{p}$ from the gauge boson mass renormalization is shown on the left. The $\gamma, \mathrm{Z}$ loop correction to the $\mathrm{Z}, \gamma$ exchange vertex is shown on the right. . . . . . . . . . . . . . . 28

2.5 Box diagrams representing the exchange of two gauge bosons (Cross terms not shown). . . . . . . . . . . . . . . . . . . . . . . 29

2.6 Two examples of diagrams contributing to the running of $\sin ^{2} \theta_{w}$. The left diagram shows a $\mathrm{Z}$ boson fluctuating into a photon via fermion loop. The right diagram shows a single W loop. . . . . . . . . . . . . 29

3.1 CEBAF schematic. . . . . . . . . . . . . . . . . . 34

3.2 The allowed optical transitions of $\Delta m_{j}= \pm 1$ in a GaAs photocathode are shown. The numbers in the circles represent the relative transition probabilities[3]. . . . . . . . . . . . . . . . . . 35

3.3 Schematic of CEBAF injector optics setup[4]. . . . . . . . . . 36 
3.4 The Wien filter causes a spin precession of the electron spin. The double Wien filter uses a pair of Wiens, Vertical and Horizontal, to orient the electron spin so as to cancel out transverse polarization due to spin procession in the linac and arcs. This allows $100 \%$ longitudinal polarization to be delivered to the experimental halls[5]. . . . . . . . 38

3.5 Schematic of cylindrical BPM as seen along the beam pipe[6]. Signal wires are shown rotated in the counter-clockwise direction to $45^{\circ}$. Hall coordinates are given by $\mathrm{X}_{H}$ and $\mathrm{Y}_{H} \ldots \ldots \ldots$

3.6 Schematic for Hall C Møller Polarimeter[7]. . . . . . . . . . . . . . . . 44

3.7 The Hall C Compton layout. D1-4 are dipole magnets, the electron beam is shown in red, black denoted the scattered electrons, and the dashed blue shows the scattered photons. . . . . . . . . . . . 45

3.8 (a) CAD drawing of the conical target cell. The conical shape of the cell accommodates the out going scattered electrons. (b)CFD simulation showing $\mathrm{lH}_{2}$ flow velocities inside the target cell[8]. . . . . 47

3.9 The defining collimator shown before installation. This collimator defines the experimental acceptance for $\mathrm{Q}_{\text {weak }}[8] \ldots$. . . . . . . . . 49

3.10 (a) $\mathrm{Q}_{\text {weak }}$ Toroidal Spectrometer installed in experimental Hall C at Jefferson Lab. (b) Current in each coil travels in a racetrack fashion creating a toroidal magnetic field. The field goes to zero at the center allowing the beam to pass unperturbed to the beam dump. . . . . . . 50

3.11 CAD diagram of shielding wall. Simulated beam envelope shown in blue. Proper design of the shielding wall was crucial to avoid interaction of the scattered envelope with the inner edge of the apertures[9]. 51

3.12 Schematic of quartz detector array. . . . . . . . . . . . . 52

3.13 Simulated beamline background with(right) and without(left) the tungsten plug. Electrons are shown in red and neutral are shown in blue. The addition of the tungsten plug drastically reduces the beamline backgrounds[8] . . . . . . . . . . . . . . . 5 54

3.14 Simple schematic of the experiment showing the 3 tracking regions. Simulated trajectories of elastically scattered electrons are shown in

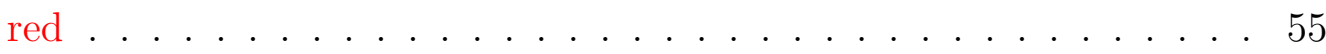

3.15 Region II HDCs installed downstream of QTOR in Hall C. . . . . . . 57

3.16 (a) Shows one completed vertical drift chamber in the lab at College of William and Mary. The VDCs were layered (b) and contained 2 wire planes, $3 \mathrm{HV}$ planes, a spacer frame, and 2 gas frames[10]. . . . 58 
3.17 Cross-section of one VDC "cell". HV planes are shown in green and the particle track is shown in blue[10]. Particles entering the cell ionize the gas causing an avalanche of charge moving towards the signal wires. The timing of these signals is used to extract track information. . . . . . . . . . . . . . . . 59

3.18 (a)Diagram of LVDS-ECL read-out and signal multiplexing in the MUX crates. A single delay line is shown for the first four multiplexed signals. (b)Typical time difference data from bench testing of the MUX crates. Each peak represents a wire in the delay chain; peak separation is $\sim 1.3 \mathrm{~ns}$ with an average $\sigma$ of $80 \mathrm{ps}[10] \ldots$. . . . . . . . 60

3.19 Schematic of trigger scintillator including dimensions[8] . . . . . . . . 61

3.20 Region III rotator. . . . . . . . . . . . . . . . . . . . . 63

3.21 The central hub of the Region III rotator. The rotator support hub was machined from 304 stainless steel; this material was chosen because of it low magnetic permeability. Steel rails, which the rotator arms ride on, were attached to the flat structures protruding from the central hub. . . . . . . . . . . . . . . . . . . . . . . . . . . . 64

3.23 Schematic showing the communications diagram for the linear motor controls. . . . . . . . . . . . . . . . . 67

3.24 Sprocketed plates placed along the rotator face were used along with a chain drive to turn the rotator. . . . . . . . . . . . . . . . 68

3.25 Motion Controls Rack. . . . . . . . . . . . . . . . . . . . 70

3.26 Laser pointers mounted on main detector support structure. . . . . . 73

4.1 The modulation coil pairs are shown in red on the far right. Each coil was driven by an amplified signal from the VMIVME-4145 wave-form generators seen in the center. A second copy of the driving signal was recorded by the main DAQ[1] . . . . . . . . . . . . . . . 78

4.2 Copper air-core coils used in the modulation system. . . . . . . . . . 78

4.3 An example beamline optics simulation to generate a $50 \mu \mathrm{m}$ offset at the target. The red arrow shows the direction of the beam. The coils are represented by $\mathrm{C} 1$ and $\mathrm{C} 2$ and show the optimal locations along the beamline to apply kicks[12]. . . . . . . . . . . . . . . . 79

4.4 Schematic of beam modulation cycle timing. Each pulse section is 512 cycles of sinusoidal function at frequency of $125 \mathrm{~Hz}$. Pulse are broken down into micro-cycles that make up a full macro-cycle. . . . 83 
4.5 The ramp signal read out by the ADCs. Because the DAQ samples at a rate of $980 \mathrm{~Hz}$ and the ramp signal has a frequency of $125 \mathrm{~Hz}$, the ADC records $\sim 8$ points per cycle. When the ramp transition falls in the middle of an ADC sample window the ramp point recorded does not match up with what is expected at that time; see data surrounded by green vertical lines. . . . . . . . . . . . . . . . . . 87

4.6 An example plot of the modulation driving signal for one coil plotted versus the ramp function is shown in red. The edge effects of the ramp signal being sampled by the ADC point recorded at an incorrect phase form a linear function that tracks through the center of the driving signal. Using a simple linearity cut many of these points (shown in black) can be removed. . . . . . . . . . . . . . . . . . 887

4.7 The phase of the BPM response is shown as a function of position along the beamline. The dashed blue lines indicate the position of the vertical and horizontal modulation coils. . . . . . . . . . 88

4.8 Correlation between TargetX and TargetX' sensitivities. . . . . . . . . 93

4.9 Correlation between BPM3c12X and TargetX sensitivities. . . . . . . 93

4.10 Correlation between BPM3c12X and TargetX' sensitivities. . . . . . . 94

4.11 Correlation between TargetY and TargetX sensitivities. . . . . . . . . 94

4.12 Correlation between Target $Y$ and Target $Y^{\prime}$ sensitivities. . . . . . . . . 95

4.13 Slug average correlation between TargetX and Target $X^{\prime}$ sensitivities. . 95

4.14 Slug average correlation between BPM3c12X and TargetX sensitivities. 96

4.15 Slug average correlation between BPM3c12X and Target $X^{\prime}$ sensitivities. 96

4.16 Slug average correlation between TargetY and TargetX sensitivities. . 97

4.17 Slug average correlation between TargetY and Target $\mathrm{X}^{\prime}$ sensitivities. . 97

4.18 TargetX response to modulation during runs with and without FFB active. . . . . . . . . . . . . . . . . . 99

4.19 Target $\mathrm{X}^{\prime}$ response to modulation during runs with and without FFB active. . . . . . . . . . . . . . . . . . 99

4.20 BPM3c12X response to modulation during runs with and without FFB active. . . . . . . . . . . . . . . . . 100

4.21 TargetY response to modulation during runs with and without FFB active. . . . . . . . . . . . . . . . . . 100

4.22 Target $Y^{\prime}$ response to modulation during runs with and without FFB active. . . . . . . . . . . . . . . . . . . 101

4.24 The same as the above plot but profiled. The pull due to the cut ramp data is much more pronounced when looking at the average versus $\mathrm{R}(\mathrm{r}(\mathrm{t})) \ldots \ldots \ldots 101$ 
4.23 The response of the TargetX BPM to $\mathrm{X}$ modulation plotted versus $R(r(t))=\sin \left(\frac{\pi}{180} r(t)+\phi\right)$, where $\mathrm{r}(\mathrm{t})$ is the ramp function. Because of the phase shift from the FFB system the response traces an ellipse in phase space; near the center of the ellipse the missing data due to the ramp cut can be seen. This missing data and the ellipsoid nature of the response-induced extra error into the sensitivities extraction and made analytical calculation of the errors on the sensitivities more difficult. . . . . . . . . . . . . . . . . . . . 102

4.25 The Run I single detector X position sensitivities over Run I versus octant number for $\mathrm{X}$ modulation. Because $\mathrm{X}$ modulation is in the horizontal plane, detectors 1 and 5 are most sensitive to this modulation type. The sinusoidal variance of the detector sensitivities, with peaks at 1 and 5, is characteristic of X-like modulation. Error bars are included but are smaller than the markers. . . . . . . . . . . . . 105

4.26 The Run I single detector Y position sensitivities over Run I versus octant number for $\mathrm{X}$ modulation. Because $\mathrm{Y}$ modulation is in the vertical plane, detectors 3 and 7 are most sensitive to this modulation type. Error bars are included but are smaller than the markers. . . . 105

4.27 The Run I single detector energy sensitivities over Run I versus octant number for E modulation. Ideally, a change in energy should be seen as a change in $\mathrm{Q}^{2}$ and effect each detector uniformly. The expected response would be a detector monopole. Error bars are included but are smaller than the markers. . . . . . . . . . . . . . . . . 106

4.28 Run I Slug-average Set 1 X sensitivities. . . . . . . . . . . . . . . . . 107

4.29 Run I Slug-average Set $1 \mathrm{X}^{\prime}$ sensitivities. . . . . . . . . . . . . . . . . 107

4.30 Run I Slug-average Set 1 BPM3c12X sensitivities. . . . . . . . . . . . 108

4.31 Run I Slug-average Set 1 Y sensitivities. . . . . . . . . . . . . . . . . 108

4.32 Run I Slug-average Set $1 \mathrm{Y}^{\prime}$ sensitivities. . . . . . . . . . . . . . . . . 109

4.33 Uncorrected yield response dipole to X modulation. . . . . . . . . . . 111

4.34 Uncorrected yield response dipole to $\mathrm{X}^{\prime}$ modulation. . . . . . . . . . . 111

4.35 Uncorrected yield response dipole to E modulation. . . . . . . . . . . 112

4.36 Uncorrected yield response dipole to Y modulation. . . . . . . . . . . 112

4.37 Uncorrected yield response dipole to YP modulation. . . . . . . . . . 113

4.38 Corrected yield response dipole to X modulation. . . . . . . . . . . . 113

4.39 Corrected yield response dipole to $\mathrm{X}^{\prime}$ modulation. . . . . . . . . . . . 114

4.40 Corrected yield response dipole to E modulation. . . . . . . . . . . . 114

4.41 Corrected yield response dipole to Y modulation. . . . . . . . . . . . 115

4.42 Corrected yield response dipole to YP modulation. . . . . . . . . . . 115 
4.43 The slug average detector widths are shown for raw, corrected via modulation, LRB, and wien average LRB for slugs 42- 59. The maximum reduction in width is achieved using the quartet level sensitivities. . . . . . . . . . . . . . . . . . 119

4.44 The beam-modulation corrected asymmetry for both IN and OUT IHWP settings. . . . . . . . . . . . . . . . . . . . . . . 123

5.1 Regressed aluminium asymmetry plotted versus slug for both IN and OUT half-wave plate settings. This does not include corrections due to polarization or backgrounds. . . . . . . . . . . . . . . . . 131

5.2 The Run I Dilution for opposing octants is shown for both normalized and unnormalized cases. The discrepancy between with and without normalisation is attributed to a difference in the reference target yields.133

5.3 Inelastic dilution from simulation as a function of QTOR setting[13]. 137

5.4 Measured average polarization by slug for Run I data. . . . . . . . . . . 144

6.1 The contribution from various corrections to the measured asymmetry. The raw asymmetry is given on the left in orange. The corrections from beam modulation and background corrections are shown in blue, and the final corrected asymmetry is shown in purple on the far right. . . . . . . . . . . . . . . . . . . . . 147

6.2 The contributions to the final error bar. . . . . . . . . . . . . . . . . . 149

6.3 The global fit of the reduced asymmetry in the forward limit to $\mathrm{Q}^{2}$ $=0.63(\mathrm{GeV} / \mathrm{c})^{2}$ is shown as the black line. The red point is the present $Q_{W}^{p}$ measurement. The yellow band represents the uncertainty of the fit. The dashed blue line shows the global fit without the measurement of $\mathrm{Q}_{W}^{p}$ presented here. The SM prediction is shown by the arrow. This fit assumes the blinding factor is close to zero. . . . . . . . . . . . . . . . . 151 
6.4 Constraints on the neutral weak vector constants: isoscalar $\left(C_{u 1}+C_{d 1}\right)$ and isovector $\left(C_{u 1}-C_{d 1}\right)$. The APV measurement shown in green mainly constrains the isoscalar combination. The blue ellipse includes the current PVES data up to $\mathrm{Q}^{2}<0.63(\mathrm{GeV} / \mathrm{c})^{2}$ as well as the value reported here. The red ellipse is the combination of PVES and APV measurements; inner ellipses are $68 \%$ confidence level and the outer are $95 \%$ confidence level. The black line represents the $\overline{M S}$ scheme SM prediction of $\sin ^{2} \theta_{W}$ [14], with the black dot indicating the SM best fit value, $\sin ^{2} \theta_{W}=0.23116$. . . . . . . . . . . . . . . . 153

A.1 The survey team used tooling balls that are attached in static locations on the VDCs to determine position in the frame of the lab. Above shows the positions and designation of each tooling ball. . . . 169 
DETERMINATION OF THE PROTON'S WEAK CHARGE VIA PARITY VIOLATING ELECTRON SCATTERING 


\section{CHAPTER 1}

\section{Introduction}

\subsection{Fundamental Symmetries of the Standard Model}

\subsubsection{Fundamental Symmetries}

The concept of fundamental symmetries has played a major role in the development of the Standard Model of particle physics and has been one of the most ubiquitous elements in the formulation of physics in the $20^{\text {th }}$ century. Conservation laws in physics are related directly to the invariance of a physical system under a transformation. In 1918, German mathematician Emmy Noether published her theorem[15] which states that in the case of a system having a continuous symmetry, there will be corresponding quantities whose values are conserved, i.e. symmetries which lead to the invariance of the action under transformation lead to conserved quantities. This is an important result because it gives insight into conservation laws intrinsic to physical systems, as well as providing a practical calculation tool for conserved quantities. Symmetries can be either discrete or continuous. An example of a discrete symmetry is reflective symmetry or parity. In the case of continuous 
symmetry, familiar examples from classical mechanics would be the invariance of a system under rotation which leads to conservation of angular momentum or translational invariance in time which gives conservation of energy. In quantum mechanics, conservation principles are tied to the commutation an of operator with the Hamiltonian. For example, consider a general operator in the Hamiltonian picture,

$$
\frac{d \mathcal{O}}{d t}=i[\mathcal{O}, H] .
$$

Here the commutator with the Hamiltonian describes the time evolution of the operator. In the event that the operator, $\mathcal{O}$, commutes with the Hamiltonian, the physical observable associated with that operator does not change with time and is therefore conserved. In Field Theories, symmetries are defined by transformations on a physical system that leave the action unchanged. The action is given in terms of the Lagrangian density by

$$
S=\int d^{4} x \mathcal{L}\left(\phi, \partial_{\mu} \phi\right)
$$

This can be simplified by looking at transformations that leave the Lagrangian density invariant. Transformations can be global or local, each of which affects the Lagrangian in different ways. As an example, consider the Dirac Lagrangian of a charged spin $1 / 2$ particle of mass $m$,

$$
\mathcal{L}=\bar{\psi}\left(i \gamma^{\mu} \partial_{\mu}-m\right) \psi
$$

A global transformation can be represented as a change of phase,

$$
\psi \rightarrow \psi^{\prime}=e^{i \alpha} \psi .
$$


It is clear that replacing $\psi \rightarrow \psi^{\prime}$ in Eq. 1.3 leaves the Lagrangian unchanged because the transformation affects all points in space-time equally. A local, or gauge, transformation is space-time dependent, however, and must be handled differently. A gauge transformation can be represented as

$$
\psi \rightarrow \psi^{\prime}=e^{i \alpha(x)} \psi
$$

Substitution of Eq. 1.5 into Eq. 1.3 finds the Lagrangian is not, at least a priori, invariant under a local gauge transformation. Gauge invariance can be imposed by introducing a gauge field with a transformation property such that the extra term is cancelled. We define the covariant derivative to be

$$
D_{\mu} \equiv \partial_{\mu}-i g A_{\mu}
$$

where $A_{\mu}$ transforms as

$$
A_{\mu} \rightarrow A_{\mu}+\frac{1}{g} \partial_{\mu} \alpha .
$$

Thus, requiring gauge invariance introduces a vector field, $\mathrm{A}_{\mu}$, that couples directly to the charged particles described by the Dirac Lagrangian. In fact, choosing the coupling constant, $g$, to represent the electric charge, $e$, we see this new vector field represents the photon field. Thus, requiring gauge invariance introduces a new vector field that couples to each particle in the theory and becomes the force carrier for the interaction. These new particles are called vector bosons. As a note, the addition of $\mathrm{A}_{\mu}$ potentially adds both a kinetic, $F_{\mu \nu} F^{\mu \nu}$, and a mass term, $m A_{\mu} A^{\mu}$, to the Dirac Lagrangian. The latter of these violates gauge invariance therefore the mass term cannot exist, the photon field is massless and infinite in range. Vector 
bosons are discussed in more detail in subsequent chapters.

As mentioned above, requiring gauge invariance introduces new vector bosons into the theory which in the case of the photon field are massless. This becomes a problem, however, when applying gauge invariance to the weak interactions, where

the charge carrier vector bosons $\left(\mathrm{Z}^{\circ}, \mathrm{W}^{ \pm}\right)$masses have been measured to be on the order of $100 \mathrm{GeV}$. The solution is spontaneous symmetry breaking. Spontaneous symmetry breaking describes a situation where the underlying laws at low energies have symmetries which are hidden. This mechanism produces the charged vector bosons $\left(\mathrm{Z}, \mathrm{W}^{ \pm}\right)$as well as giving mass to the fermions. This is know as the Higgs Mechanism and it plays a crucial part in our understanding of the Standard Model.

\subsubsection{Standard Model Overview}

At present the interactions and constituents that underlay the observable universe have been reduced to a handful of physical laws defined by the Standard Model (SM). The SM is the theory of the electromagnetic, weak, and strong forces, as well as the particles that make up the building blocks of matter, and how these forces mediate the subatomic world. The SM in its current form was mostly finished in the early 1970's starting with the confirmation that the proton was made up of smaller constituents[16, 17]; at the time these were called partons, however they were later identified as the up and down quarks. The early theoretical development of the SM started with Glashow's 1961 combination[18] of the electromagnetic and weak interactions to form the $S U(2)_{L} \times \mathrm{U}(1)_{\mathrm{Y}}$ gauge group, which created electroweak theory. After the addition of the Higgs[19] by Weinberg and Salam[20, 21] in 1967, the electroweak theory in its current manifestation was mostly complete. The addition of the quark model proposed by Gell-Man[22] and Zweig[23] added 
$\mathrm{SU}(3)_{\mathrm{C}}$ color symmetry defining the current form of the SM as operating under the $\mathrm{U}(1)_{\mathrm{Y}} \times \mathrm{SU}(2)_{\mathrm{L}} \times \mathrm{SU}(3)_{\mathrm{C}}$ gauge symmetry.

Since the coming together of electroweak theory and the quark model defined the current SM, experimental tests have been used to systematically verify its validity. In the mid-1970's the discovery of neutral-weak currents generated via $Z^{\circ}$ boson exchange[24, 25] helped confirm electroweak unification for which Glashow, Salam, and Weinberg later shared the 1979 Nobel prize. The Prescott experiment[26] performed at the Stanford Linear Accelerator, in the late 1970's, saw the experimental measurement of the SM predicted parity-violating asymmetry in inelastic electronDeuteron scattering; this was an important step in the wide acceptance of the SM. The construction of the proton-antiproton collider at CERN in the late seventies opened the door to the production of particles two orders of magnitude above the proton mass. This lead to the experimental discovery of of the $\mathrm{W}^{ \pm}[27,28]$ and $\mathrm{Z}^{\circ}[29,30]$ and was of fundamental importance in validating a crucial element of the SM; this discovery earned Carlo Rubbia and Simon van de Meer the 1984 Nobel Prize. Over the course of the last 30 years experimental verification of the SM has continually mounted, and with the recent discovery of what is likely to be the Higgs $[31,32]$ at CERN, the final building block of the SM has been experimentally observed and verified. To date, the SM has been successful in explaining nearly all of high-energy experimental data and has fostered a rich program of experimental programs testing fundamental theories and searching for evidence of possible extensions.

The SM describes the laws governing the building blocks of matter and their interactions (Fig. 1.1). The SM describes all matter as being made up of 12 spin $1 / 2$ particles known as fermions and 4 vector bosons that govern their interactions; a spin-0 scalar boson, the Higgs, gives the particles mass. The fermions are divided 


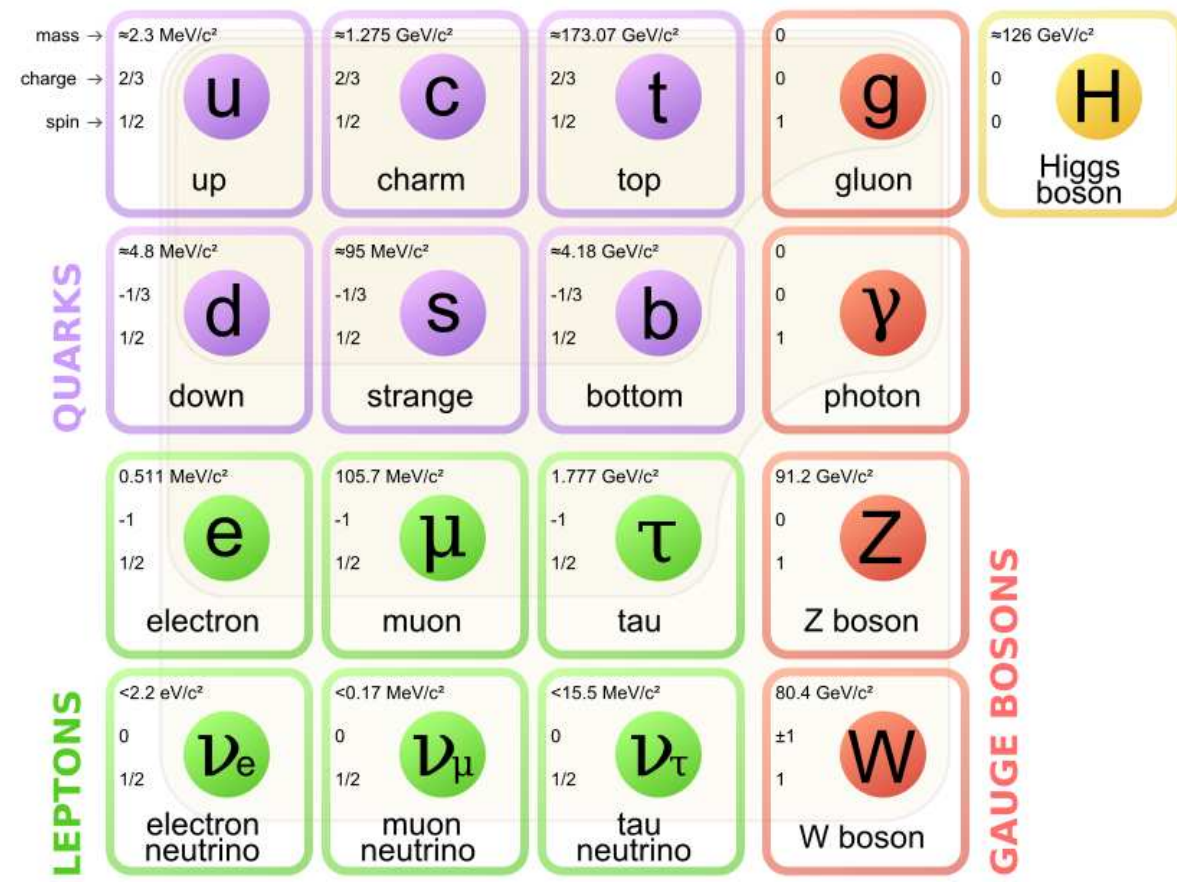

FIG. 1.1: The Standard Model of Particle Physics as presently determined. Fermions (1/2 integer spin) are divided into three generations with similar properties and increasing mass. Fermions are divided into leptons (do not interact via the strong coupling) and quarks. Force carriers are represented by the integer spin bosons to the right. Together these make up the Standard Model and have been extremely successful in explaining experimental data over the last 50 years[1].

into quarks and leptons. There are 6 leptons, three of which carry a negative integral charge: electron $(e), \operatorname{muon}(\mu), \operatorname{tau}(\tau)$. Neutrinos are neutral in charge and designated as: electron neutrino $\left(\nu_{e}\right)$, muon neutrino $\left(\nu_{\mu}\right)$, tau neutrino $\left(\nu_{\tau}\right)$. Each lepton has the intrinsic properties of mass, charge, and spin. The quarks are defined in a similar manner with the first generation being defined as $\operatorname{up}(u)$ and $\operatorname{down}(d)$, the second being charm $(c)$ and strange $(s)$, and the third being top $(t)$ and $\operatorname{bottom}(b)$. Each quark has an associated intrinsic mass, charge, spin, and color charge. Each generation of lepton is increasingly more massive, with the first generation being the lightest; the heavier generations are in fact not stable and quickly decay with short lifetimes. Interestingly, because the first generation of charged fermions do not 
decay, they make up all baryonic (formed from a bound state of 3 quarks) matter in the universe.

The force carriers of the SM define how particles interact with each other. In the SM, these force carriers are the spin-1 vector bosons. The charged interaction or the electromagnetic force between both leptons and quarks is mediated by the photon. Classically, this coupling is what we think of as the electric charge; with the unification of the electromagnetic and weak gauge groups into electroweak theory, charge actually becomes a function of both weak isospin and weak hypercharge. This is discussed more in section 1.2.2. The weak charge is mediated via three massive vector bosons: $\mathrm{Z}^{\circ}, \mathrm{W}^{+}$, and $\mathrm{W}^{-}$. Due to their mass, each force carrier's interaction range is short, as opposed to that of the photon, which is massless and has an infinite range. The neutrinos, being neutral particles with no charge, interact only via the weak charge making them difficult to detect. The strong force, which is responsible for quark binding within the nucleus, is mediated by the eight massless gluons via coupling to color charge. Leptons, being devoid of color charge, do not interact via the strong force; this is what separates the fermions from the leptons. Interestingly, unlike leptons which can be observed as free particles, quarks are only found in bound states. Each quark has a color charge (Red, Blue, Green), and interacts under the strong, weak, and electromagnetic interactions. The bound states of quarks come in colorless combinations of three (baryons), pairs (mesons), and possibly other combinations. The fact that quarks are only found in bound states is a demonstration of color confinement; confinement also has the interesting property that, unlike the electroweak force, the strength of the coupling increases as the distance between quarks increases.

Despite the obvious success of the SM in the explanation of the interactions and the constituents making up the universe, it has a number of significant failures. One 
of the most significant shortcomings is that there is no way of reliably describing the canonical theory of gravitation, General Relativity, in terms of modern quantum field theory. Other problems such as a lack of explanation of the matter/anti-matter asymmetry in the universe and lack of a proper dark matter candidate particle have lead to the exploration of Beyond the Standard Model(BTSM) physics, as well as experimental programs centred around testing SM observables in an effort to find discrepancies; the discrepancies could give important insight into the nature of new physics.

\section{$1.2 \quad$ Electroweak Theory}

\subsubsection{Discrete Symmetry and Spin}

Mentioned briefly in the beginning of the chapter (Subsec. 1.1.1) was the idea of discrete symmetries which play an important role in the Standard Model; specifically parity. Parity describes the way a system behaves under a spatial transformation, $(\mathrm{x}, \mathrm{y}, \mathrm{z}) \rightarrow(-\mathrm{x},-\mathrm{y},-\mathrm{z})$. This is often referred to as mirror symmetry as it is a reflection through the origin and manifests in a similar way to looking in a mirror. Originally, parity was thought to be a conserved quantity in the SM; experimental evidence in both the electromagnetic and strong interactions indicated that parity was conserved. In 1956 T. D. Lee and C. N. Yang, while looking at the question of parity conservation in $\beta$, hyperon, and meson decays, pointed out that there was no a priori reason why parity should be conserved in the weak interactions. In fact there was no experimental evidence for (or against) parity violation in the weak interactions [33]. Subsequently, at the National Bureau of Standards, an experiment using $\beta$-decay in ${ }^{60}$ Co nuclei was carried out by C.S. Wu and colleagues [34] to test 
the theory set forth in Yang and Lee's paper. The idea of the experiment was to look at the decay distribution of ${ }^{60} \mathrm{Co}$ nuclei in a magnetic field. If the weak interactions, which mediated the decay process, conserved parity then the measured electron emission should be the same for both ${ }^{60}$ Co spin aligned and anti-aligned with the magnetic field. What was found was a measurable asymmetry in the detected emission indicating a preferred direction in the decay process. This provided the first experimental evidence of parity violation in the weak interactions and lead to a 1957 Nobel prize for Yang and Lee.

An important property to consider, especially in the discuss parity and the weak interactions, is the way in which "handedness" behaves under parity transformation. The spin of a particle can be used to define the "handedness" of a particle and is often referred to as the helicity or in the massless, relativistic limit, the chirality. Helicity describes the orientation of the spin vector with respect to the momentum of the particle. For a right-handed particle, the momentum and the spin would be in the same direction, and for left-handed would be opposite of each other. A particle's chirality is more subtle, and is defined by the chiral "projection operator" shown in Eq. 1.8 and 1.9.

$$
\begin{aligned}
& u_{L}(p)=\frac{1}{2}\left(1-\gamma^{5}\right) u(p) \\
& u_{R}(p)=\frac{1}{2}\left(1+\gamma^{5}\right) u(p)
\end{aligned}
$$

Here the projection operator acts on the particle spinor returning a left(right)handed chiral particle. A key difference between helicity and chirality can be understood considering a Lorentz boost. For a massive particle it is possible to boost a left-handed particle such that the helicity is reversed. This leads to the situation where the particle doesn't interact the same in all reference frames. This is not true of the chirality which is an intrinsic property of the particle and invariant 
under change of reference frame. As mentioned above, in the limit where the particle is massless and relativistic, helicity and chirality become the same. In further discussion contained in this document, only the case of massless, ultra-relativistic electrons will be considered.

\subsubsection{Electroweak Unification}

As mentioned in Sec.1.1.2, the vector bosons, $\mathrm{W}^{ \pm}$and $\mathrm{Z}^{\circ}$, act as the force carriers of the weak force. The vector bosons also interact under the electromagnetic force. The fact that the force carriers have a charge associated with both the electromagnetic force as well as the weak force hints at the unification of the electromagnetic and weak forces. The unification of both the weak and electromagnetic interactions into a single theoretical framework, in which they would appear as different manifestations of a single theory, was the goal of Glashow's early work [18]. The addition of later work by Weinberg and Salam[20, 21] lead to the eventual development of the current electroweak theory. In the following section I will briefly discuss some of the underlying ideas of electroweak unification.

The weak and electromagnetic theories are mathematically unified under the $S U(2)_{L} \times U(1)_{Y}$ gauge group. Recalling the previous discussion of gauge invariance of the Lagrangian (Sec. 1.1.1), we can define the covariant derivative for the electroweak Lagrangian to be,

$$
D_{\mu} \psi=\left(\partial_{\mu}+\frac{i g}{2} \tau^{i} W_{\mu}^{i}+\frac{i g^{\prime}}{2} Y B_{\mu}\right) \psi
$$

Here $W_{\mu}$ represents the charged vector boson triplet required for gauge invariance under $S U(2)_{L}$, and $\tau^{i}$, known as the Pauli spin matrices, are generators of the weak isospin symmetry. The vector boson singlet is given by $B_{\mu}$, and the generator of the 
$U(1)_{Y}$ symmetry is given by the hypercharge, $\mathrm{Y}$. The couplings for the electroweak theory are given for $S U(2)_{L}$ and $U(1)_{Y}$ as $g$ and $g^{\prime}$ respectively. Under the unified theory the electric charge is redefined in terms of weak isospin and the new $U(1)$ symmetry hypercharge as

$$
Q=T^{3}+\frac{Y}{2}
$$

In a sense this unifies the electromagnetic and weak interaction, albeit with two independent coupling strengths. Using the covariant derivative (1.10), the Lagrangian for the electroweak theory can be constructed; mass terms of the form $m \bar{\psi} \psi$ are excluded due to failing gauge invariance. The fermion masses in the electroweak theory are generated by spontaneous symmetry breaking via the Higgs Mechanism in which the degrees of freedom of the scalar Higgs field are "absorbed" by the massive gauge bosons. In short, a set of complex scalar fields, the Higgs, can be introduced, resulting in a breaking of the $S U(2)$ global symmetry. As a result, the theory produces three massive vector bosons, $W^{ \pm}$and $Z^{\circ}$, and one massless boson, the photon $(\gamma)$, as well as giving mass to the fermions. The Lagrangian describing the new scalar fields is given by

$$
\mathcal{L}=\left(D_{\mu} \Phi\right)^{\dagger}\left(D^{\mu} \Phi\right)-\mu^{2} \Phi^{\dagger} \Phi+\frac{\lambda^{2}}{4}\left(\Phi^{\dagger} \Phi\right)^{2}
$$

where

$$
\Phi=\left(\begin{array}{l}
\phi^{+} \\
\phi^{\circ}
\end{array}\right)
$$

is the complex scalar Higgs doublet. The choice of $\mu^{2}$ here matters. If $\mu^{2}>0$ is chosen the theory returns the standard QED theory with a massless photon and a charged scalar. Choosing $\mu^{2}<0$ however results in the "mexican hat" potential 
which has a non-zero vacuum expectation value $(\langle\Phi\rangle \neq 0)$, i.e. the symmetry is broken. Minimizing the potential in the above Lagrangian and choosing an arbitrary ground state breaks the $S U(2)$ symmetry. This choice is important because the minima lie on a circle of radius $\mu^{2} / \lambda^{2}$ and therefore there are an infinite number of solutions. Expanding 1.12 above allows for identification of the charged vector bosons as

$$
W^{ \pm}=\frac{1}{\sqrt{2}}\left(W^{1} \mp i W^{2}\right)
$$

and the neutral vector bosons as

$$
\begin{aligned}
& Z_{\mu}^{0}=\cos \theta_{w} W_{\mu}^{3}-\sin \theta_{w} B_{\mu} \\
& A_{\mu}=\sin \theta_{w} W_{\mu}^{3}+\cos \theta_{w} B_{\mu} .
\end{aligned}
$$

Here the physical vector bosons we see in experiment, and which gain mass through symmetry breaking, are defined as a mixing between the neutral bosons $W_{\mu}^{3}$ and $B_{\mu}$. The parameter $\theta_{w}$ is the "weak mixing angle" and describes the mixing between the neutral vector boson couplings. The masses acquired by the vector bosons through the Higgs mechanism are give by:

$$
\begin{aligned}
m\left(W_{\mu}^{ \pm}\right) & =\frac{1}{4} g^{2} \nu^{2} \\
m\left(Z_{\mu}^{0}\right) & =\frac{1}{4}\left(g^{2}+g^{2}\right) \nu^{2} \\
m\left(A_{\mu}\right) & =0
\end{aligned}
$$

Following this further, the vector boson masses can be related as

$$
\cos \theta_{w}=\frac{m_{W}}{m_{Z}}
$$


This answers one of the central questions in electroweak physics; why are the masses of the $W^{ \pm}$and $Z^{0}$ different? It is also important to note that under unification the couplings of electroweak theory are not independent. In the interest of foreshadowing, $g$ and $g^{\prime}$ can be related as

$$
\sin ^{2} \theta_{W}=\frac{g^{\prime 2}}{g^{2}+g^{\prime 2}} .
$$

The term $\sin ^{2} \theta_{W}$ is a center piece of the work in this thesis and will be discussed at length in subsequent chapters.

Lastly, the fermions gain mass via interaction with the Higgs. Considering the general $\mathrm{SU}(2) \times \mathrm{U}(1)$ Yukawa coupling of a scalar particle interacting with fermions along with the Higgs doublet defined by

$$
\Phi=\left(\begin{array}{c}
0 \\
\nu+h(x)
\end{array}\right)
$$

where $h(x)$ is a small perturbation about the ground state. The interaction Lagrangian for the Higgs coupling to the fermions is given by

$$
\mathcal{L}_{Y U}=g^{(e)} \bar{\psi}_{L} \Phi e_{R}+\text { h.c. }
$$

where $\psi_{L}$ describes the $\mathrm{SU}(2)$ electron doublet and $g^{(e)}$ represents the Yukawa coupling. For simplicity I have only included minimal terms in the interaction Lagrangian; this can be expanded to include all fermions. Expanding upon this, the fermions masses can be seen as coefficients of terms that are quadratic in the fields. It is interesting to note that the actual masses of the fermions are not predicted by the theory and are only input parameters. The above work only shows how the 
masses are formed under the theory. 


\section{CHAPTER 2}

\section{The $\mathrm{Q}_{\text {weak }}$ Experiment}

\subsection{Experimental Motivation}

The Standard Model (SM), while being successful in describing the fundamental interactions found in nature, is thought to be an effective low-energy theory of a more fundamental underlying physics. There are two complementary methods of searching for new physics: that of high energy experiments which strive to excite matter into new forms, and that of precision experiments which aim to measure observables in the SM that are precisely predicted. Historically, precision experiments have been crucial in studying the structure of the nucleon and understanding the electroweak interaction. The weak charge of the proton, $Q_{w}^{p}=1-4 \sin ^{2} \theta_{w}$ (tree level), which is the neutral-weak analog of the proton's electric charge[35], is both precisely predicted and moderately suppressed in the SM. Measurements made at the Z-pole have done an impressive job providing constraints and verifying predictions of the $\sin ^{2} \theta_{w}$ at high energy. A lesser studied area, which has great potential, is measurement of $\sin ^{2} \theta_{w}$ at low $\mathrm{Q}^{2}$. The SM predicts a shift of $\Delta \sin ^{2} \theta_{w}=0.007$ at 
low $Q^{2}$ with respect to the Z-pole value. This shift comes about due to the energy dependence of the weak coupling; as $Q^{2}$ goes higher, radiative corrections shift the value from the measured value at the pole. Significant deviation from the theoretically predicted value would be a strong indication of new physics, while precise agreement would provide an important stand-alone confirmation of the SM. Thus, a measurement of $Q_{w}^{p}$ provides an excellent candidate for indirect searches of new physics; specifically parity-violating (PV) physics in the coupling between electrons and light quarks.

The $Q_{\text {weak }}$ experiment, which ran at the Thomas Jefferson National Accelerator Facility (JLab) from November 2010 to May 2012, provides the first direct determination of $Q_{w}^{p}$ via a precise measurement of the PV asymmetry in $\overrightarrow{\mathrm{e}} p$ scattering at low momentum transfer $\left(Q^{2} \approx 0.025 \mathrm{GeV}^{2}\right)$. The choice of low momentum transfer and the use of parity-violating electron scattering (PVES) world data, which helps to constrain errors, allows for a theoretically clean extraction of $Q_{w}^{p}$. First results were recently released using only $4 \%$ of our full data set[36]. Precise measurement of the weak-charge, which can be written in terms of the vector quark weak charges as $Q_{w}^{p}=-2\left(2 C_{1 u}+C_{1 d}\right)$, also provides an important complement to precision atomic parity-violation (APV) experiments. APV experiments on ${ }^{133} \mathrm{Cs}[37]$ provide access to a different linear combination of the vector quark weak charges that can be used to separate and determine $C_{1 u}$ and $C_{1 d}$. The following chapter aims to lay out the basic theoretical framework and possible implication of the $Q_{\text {weak }}$ experiment's measurement. 


\section{$2.2 \quad$ Parity-Violating $e+p$ Scattering}

\subsubsection{Neutral-Weak Interaction}

The neutral-weak interaction is of fundamental importance to PVES experiments. As explained in Sec. 1.2, the interactions in the SM are mediated by the transfer of gauge bosons. The neutral-weak interactions describe interaction via $\gamma$ or $Z^{0}$ bosons, and are considered neutral because the interaction does not affect the charge of the incoming and outgoing particles. The neutral-weak current for both the $\gamma$ and $Z^{0}$ are given by

$$
\begin{gathered}
\mathcal{J}_{\mu}^{N C}=\frac{g}{\cos \theta_{W}}\left[\mathcal{J}_{\mu}^{3}-\sin ^{2} \theta_{W} \mathcal{J}_{\mu}^{E M}\right] \\
\mathcal{J}_{\mu}^{E M}=\bar{\psi} Q \gamma_{\mu} \psi
\end{gathered}
$$

Here $\mathcal{J}^{E M}$ is the electromagnetic current and $\mathcal{J}^{3}$ is the current associated with the third component of the $\mathrm{SU}(2)_{L}$ isospin triplet. The mixing intrinsic to the electroweak interaction, explained in the previous chapter, is manifest in the make up of the neutral-weak current being in terms of $\mathcal{J}^{3}\left(\mathrm{SU}(2)_{L}\right)$ and $\mathcal{J}^{E M}\left(\mathrm{U}(1)_{Y}\right)$. For simplicity, only the electron fields will be considered. Equations 2.1 and 2.2 can be expanded as

$$
\mathcal{J}_{\mu}^{N C}=\frac{g}{\cos \theta_{W}}\left[\bar{\psi}^{e} \gamma_{\mu}\left(\frac{1}{2}\left(1-\gamma^{5}\right)+Q \sin ^{2} \theta_{W}\right) \psi^{e}\right]
$$

It is instructive to write Eq. 2.3 in terms of left and right handed fields

$$
\mathcal{J}_{\mu}^{N C}=\frac{g}{\cos \theta_{W}}\left[\bar{\psi}_{L}^{e} \gamma_{\mu}\left(-\frac{1}{2}+Q \sin ^{2} \theta_{W}\right) \psi_{L}^{e}+\bar{\psi}_{R}^{e} \gamma_{\mu}\left(Q \sin _{\theta_{W}}^{2}\right) \psi_{R}^{e}\right]
$$


where the substitutions,

$$
\begin{aligned}
& \psi_{L}=\frac{1}{2}\left(1-\gamma^{5}\right) \psi \\
& \psi_{R}=\frac{1}{2}\left(1+\gamma^{5}\right) \psi
\end{aligned}
$$

have been made. Here we can see one of the most important intrinsic properties of the neutral-weak current; the property which is the basis of PVES experiments. The neutral-weak force interacts differently between left-handed and right-handed particles, and it therefore violates parity. It is important to note that neither the vector $\left(\gamma^{\mu}\right)$ nor the axial $\left(\gamma^{5}\right)$ part of 2.3 violates parity. The vector part flips sign

under parity while the axial part does not; considering the square of the scattering amplitude neither pure vector nor pure axial would violate parity. It is the interference of the $\mathrm{V}$-A coupling that is the basis of the neutral-weak current violating parity. The general form of Eq. 2.3 is given in terms of the vector and axial parts as

$$
\mathcal{J}_{\mu}^{N C}=\frac{g}{\cos \theta_{W}} \bar{\psi}^{e} \gamma_{\mu}\left(c_{V}^{f}+c_{A}^{f} \gamma^{5}\right) \psi^{e},
$$

where $c_{V}^{e}$ and $c_{A}^{e}$ and the vector and axial couplings respectively and $f$ is the fermion flavor. Comparing this with Eq. 2.3 above the couplings can be identified as $c_{V}^{e}=$ $-\frac{1}{2}+2 \sin _{\theta_{W}}^{2}$ and $c_{A}^{e}=-\frac{1}{2}$.

The results for the $c_{V}$ and $c_{A}$ couplings depend on what flavour of fermion is interacting with the $Z^{0}$. The results for each flavour as well as the electric charge are given in Table. 2.1.

\subsubsection{Low Momentum Electron Scattering}

At low momentum, the tree-level scattering amplitude of electron-proton scattering is mediated by either the $\gamma$ or $\mathrm{Z}^{0}$. The tree-level scattering amplitude in terms 


\begin{tabular}{|l|c|c|c|}
\hline Fermion Flavor & $Q_{f}$ & $c_{A}^{f}$ & $c_{V}^{f}$ \\
\hline$\nu_{e}, \nu_{\mu}, \nu_{\tau}$ & 0 & $\frac{1}{2}$ & $\frac{1}{2}$ \\
\hline$e, \mu, \tau$ & -1 & $-\frac{1}{2}$ & $-\frac{1}{2}+2 \sin ^{2} \theta_{W}$ \\
\hline$u, c, t$ & $\frac{2}{3}$ & $\frac{1}{2}$ & $\frac{1}{2}+\frac{4}{3} \sin ^{2} \theta_{W}$ \\
\hline$d, s, b$ & $-\frac{1}{3}$ & $-\frac{1}{2}$ & $-\frac{1}{2}+\frac{2}{3} \sin ^{2} \theta_{W}$ \\
\hline
\end{tabular}

TABLE 2.1: The vector and axial couplings interacting with $Z^{0}$ are shown for each flavor of lepton.

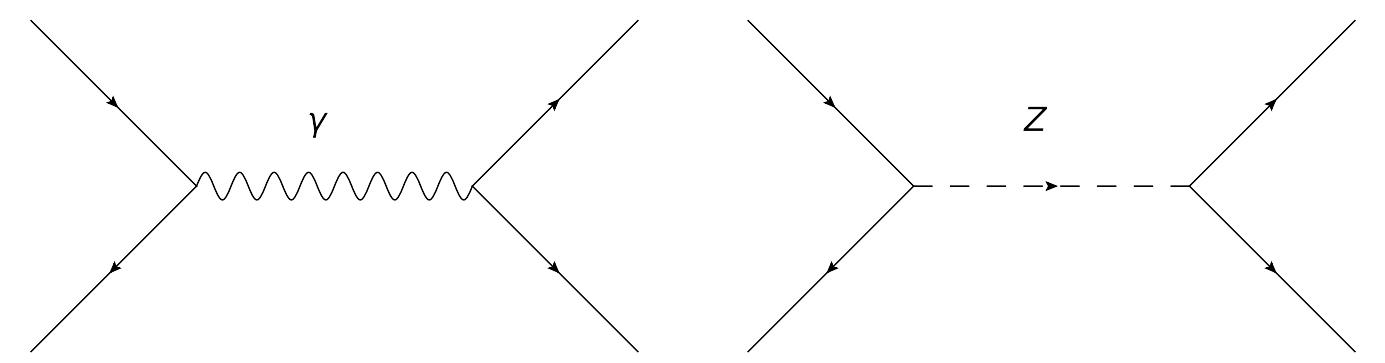

FIG. 2.1: Tree level diagrams for ep scattering in the case of the electromagnetic and neutral-weak interactions.

of currents is

$$
\mathcal{M}_{\gamma, Z}=\mathcal{J}_{\mu}^{e, \gamma} \mathcal{J}_{p, \gamma}^{\mu}+\mathcal{J}_{\mu}^{e, p}\left(V_{p, Z}^{\mu}-A_{p, Z}^{\mu}\right)
$$

The electron currents for both the electromagnetic and neutral weak interactions were derived in the previous section (Sec. 2.2.1) and are shown in Eq. 2.9a and Eq. $2.9 \mathrm{~b}$ in terms of the vector and axial couplings.

$$
\begin{aligned}
\mathcal{J}_{\mu}^{e, \gamma} & =-e \bar{u} \gamma_{\mu} u \\
\mathcal{J}_{\mu}^{e, Z} & =\bar{u} \gamma_{\mu}\left(g_{V}+g_{A} \gamma_{5}\right) u
\end{aligned}
$$

The interaction at the proton's vertex is more complicated because, unlike the electron, the proton has an internal structure and so the amplitude involves complicated interactions of the force carrier with the valence and sea quarks. Instead we represent the current at the proton's vertex in terms of structure functions. These structure functions, or form factors, give the properties of the particle interactions 
at a given momentum transfer without having to include the underlying physics directly. This is important because often the calculations of the underlying physics cannot be done at present. The proton's electromagnetic current can be written as

$$
\mathcal{J}_{p, \gamma}^{\mu}=\bar{u}\left[\gamma^{\mu} F_{1}^{N}\left(Q^{2}\right)+\sigma^{\mu \nu} \frac{q_{\nu}}{2 M} F_{2}^{N}\left(Q^{2}\right)\right] u
$$

where $F_{1}$ and $F_{2}$ are the Dirac and Pauli form factors respectively. These form factors contain complete information about the elastic electric and magnetic interaction of the nucleon as a function of the four momentum transfer $\mathrm{Q}^{2}$. The Sachs electric and magnetic form factors are given in terms of the Dirac and Pauli form factors as

$$
\begin{aligned}
& G_{E}\left(Q^{2}\right)=F_{1}\left(Q^{2}\right)-\frac{Q^{2}}{4 M^{2}} F_{2}\left(Q^{2}\right) \\
& G_{M}\left(Q^{2}\right)=F_{1}\left(Q^{2}\right)+F_{2}\left(Q^{2}\right) .
\end{aligned}
$$

Some intuition about the Sachs form factors can be gained by considering scattering in the Breit frame, i.e. the frame in which the initial and final state nucleon's momenta have the same magnitude. In this reference frame the Sachs electric form factor $G_{E}$ can be interpreted as the Fourier transform of the charge distribution; the magnetic form factor gives the Fourier transform of the magnetization distribution in the proton, which at $Q^{2} \rightarrow 0$ give the anomalous magnetic moment, $\mu_{p}$.

In addition to the electromagnetic coupling of the proton, the neutral-weak coupling must also be considered. The vector $\left(V_{p, Z}\right)$ and axial $\left(A_{p, Z}\right)$ combinations 
of the neutral-weak form factors are

$$
\begin{aligned}
& V_{p, Z}^{\mu}=\bar{u}\left[\gamma^{\mu} F_{1}^{Z}\left(Q^{2}\right)+i \sigma^{\mu \nu} \frac{q_{\nu}}{2 M} F_{2}^{Z}\left(Q^{2}\right)\right] u \\
& A_{p, Z}^{\mu}=\bar{u}\left[\gamma^{\mu} \gamma^{5} G_{A}^{Z}\left(Q^{2}\right)\right] u
\end{aligned}
$$

The neutral-weak vector form factors are given by $F_{1}^{Z}$ and $F_{2}^{Z}$ and the axial contribution is given in terms of the axial form factor $\left(G_{A}^{Z}\right)$. The neutral-weak form factors are less experimentally known compared to the electromagnetic form factors. It is helpful, given the internal structure of the nucleon, to write the neutral-weak form factors in terms of the sum of their quark contributions. Neglecting contributions from the more massive quarks, the form factors can be written as

$$
\begin{aligned}
G_{E, M}^{\gamma, p} & =\frac{2}{3} G_{E, M}^{u}-\frac{1}{3} G_{E, M}^{d}-\frac{1}{3} G_{E, M}^{s} \\
G_{E, M}^{Z, p} & =\frac{1}{2}\left(1-\frac{1}{8} \sin ^{2} \theta_{w}\right) G_{E, M}^{u}-\frac{1}{2}\left(1-\frac{4}{3} \sin ^{2} \theta_{w}\right) G_{E, M}^{d}-\frac{1}{2}\left(1-\frac{4}{3} \sin ^{2} \theta_{w}\right) G_{E, M}^{s} \\
G_{A}^{Z, p} & =G_{A}^{s}+G_{A}^{d}-G_{A}^{u}
\end{aligned}
$$

where $G_{E, M}^{i}$ is the $i^{t h}$ quark form factor. The nucleon flavor forms factors above carry contributions from both the valence and sea quarks; the exception is the strange form factor which only a contribution from the quark sea given the fact that the nucleon has no net strangeness. At low energies the proton and neutron can be thought of as different manifestations of the same particle; this defines isospin 
symmetry. Using isospin symmetry,

$$
\begin{aligned}
G_{E, M}^{u, p} & =G_{E, M}^{d, n} \\
G_{E, M}^{u, n} & =G_{E, M}^{d, p}
\end{aligned}
$$

to rewrite 2.13 , we can define the neutral-weak form factor in terms of the electromagnetic form factors of the proton and neutron as

$$
G_{E, M}^{Z, p}=\left(1-4 \sin ^{2} \theta_{w}\right) G_{E, M}^{\gamma, p}-G_{E, M}^{\gamma, n}
$$

\subsubsection{Physics Asymmetry}

The elastic process of an electron scattering from a proton contains contributions from both the electromagnetic and neutral-weak interactions. At low momentum transfer, the tree-level total invariant amplitude scattering can be expressed as the square of the sum of the diagrams for $\gamma$ and $Z^{0}$ exchange,

$$
\begin{aligned}
\left|\mathcal{M}_{e p}\right|^{2} & =\left|\mathcal{M}_{\gamma}+\mathcal{M}_{\mathcal{Z}}\right|^{2} \\
& =\left|\mathcal{M}_{\gamma}\right|^{2}+\left|\mathcal{M}_{\mathcal{Z}}\right|^{2}+2 \Re\left(\mathcal{M}_{\gamma}^{*} \mathcal{M}_{\mathcal{Z}}\right) .
\end{aligned}
$$

Because $\mathcal{M}_{\gamma} \gg \mathcal{M}_{Z}$, the electromagnetic amplitude dominates in terms of contribution, however because the neutral weak current doesn't conserve parity it can be isolated by defining the difference between the scattering amplitudes, or the parity-violating asymmetry. The PV asymmetry $\left(A_{e p}\right)$ for longitudinally polarized electrons scattering from unpolarized protons can be defined as the difference between the scattering cross section of positive and negative helicity electrons over the 
total scattering cross section,

$$
\begin{gathered}
A_{e p}=\frac{d \sigma_{L}-d \sigma_{R}}{d \sigma_{L}+d \sigma_{R}} . \\
A_{e p} \approx \frac{2 \operatorname{Re}\left(\mathcal{M}_{\gamma}^{*} \mathcal{M}_{\mathcal{Z}}\right)}{\left|\mathcal{M}_{\gamma}\right|^{2}}
\end{gathered}
$$

The crucial piece of the asymmetry lies in the numerator. The asymmetry of $\mathcal{M}_{\gamma}^{2}$ disappears due to parity conservation, leaving only the $\mathcal{M}_{Z}^{2}$ amplitude and the interference term. Here the interference term dominates and the asymmetry is approximated by

$$
A_{e p} \approx \frac{2 \operatorname{Re} \mathcal{M}_{\gamma}^{*}\left(\mathcal{M}_{Z, L}-\mathcal{M}_{Z, R}\right)}{\left|\mathcal{M}_{\gamma}\right|^{2}}
$$

The fact that the weak interactions violate parity allows us to isolate the neutral weak contribution which would otherwise be lost in the electromagnetic scattering amplitude. At tree level this can be expressed in terms of the Sachs electromagnetic form factors and grouped into three pieces as

$$
A=\frac{G_{F} Q^{2}}{2 \sqrt{2} \pi \alpha}\left[A_{E}+A_{M}+A_{A}\right]
$$

where the electromagnetic, magnetic, and axial asymmetries define groups of Sach's form factors,

$$
\begin{aligned}
A_{E} & =\frac{\epsilon G_{E}^{\gamma} G_{E}^{Z}}{\epsilon\left(G_{E}^{\gamma}\right)^{2}+\tau\left(G_{M}^{\gamma}\right)^{2}} \\
A_{M} & =\frac{\tau G_{M}^{\gamma} G_{M}^{Z}}{\epsilon\left(G_{E}^{\gamma}\right)^{2}+\tau\left(G_{M}^{\gamma}\right)^{2}} \\
A_{A} & =\frac{\frac{1}{2} \sqrt{\tau\left(1-\epsilon^{2}\right)(1+\tau)} G_{M}^{\gamma} G_{A}^{Z}}{\epsilon\left(G_{E}^{\gamma}\right)^{2}+\tau\left(G_{M}^{\gamma}\right)^{2}}
\end{aligned}
$$


Both $A_{E}$ and $A_{M}$ terms arise due to the axial electron current coupling to the vector current of the proton, whereas the axial term, $A_{A}$, arises from the vector coupling of the $Z^{0}$ to the electron and the axial coupling to the nucleon. Expanding Eq. 2.20 in terms of the form factors,

$$
A_{e p}=A_{0}\left[\frac{\varepsilon G_{E}^{\gamma} G_{E}^{Z}+\tau G_{M}^{\gamma} G_{M}^{Z}-\left(1-4 \sin ^{2} \theta_{W}\right) \varepsilon^{\prime} G_{M}^{\gamma} G_{A}^{Z}}{\varepsilon\left(G_{E}^{\gamma}\right)^{2}+\tau\left(G_{M}^{\gamma}\right)^{2}}\right]
$$

where

$$
A_{0}=\frac{-G_{F} Q^{2}}{4 \pi \alpha \sqrt{2}}, \quad \varepsilon=\frac{1}{1+2(1+\tau) \tan ^{2} \frac{\theta}{2}}, \quad \text { and } \quad \varepsilon^{\prime}=\sqrt{\tau(1+\tau)\left(1-\varepsilon^{2}\right)}
$$

are kinematic quantities, $G_{F}$ the Fermi constant, $\sin ^{2} \theta_{W}$ the weak mixing angle, $-Q^{2}$ the four-momentum transfer squared, $\alpha$ the fine structure constant, $\tau=$ $Q^{2} / 4 M^{2}, M$ the proton mass, and $\theta$ the laboratory electron scattering angle. In the forward angle and low momentum limit this can be rewritten in a more simple form. Considering the kinematic variables in the limit of $Q^{2} \rightarrow 0$ it is straightforward to see that $\varepsilon \rightarrow 1, \varepsilon^{\prime} \rightarrow 0$, and $\tau \rightarrow 0$. This reduces Eq. 2.22 to

$$
A_{e p}=A_{0}\left[\frac{G_{E}^{Z}}{G_{E}^{\gamma}}\right]
$$

Replacing $G_{E}^{Z}$ above with Eq. 2.15 and grouping next-to-leading order terms into the hadronic term $\mathrm{B}\left(Q^{2}, \theta\right)$ the asymmetry can be written simply as

$$
A_{e p} / A_{0}=Q_{w}^{p}+Q^{2} B\left(Q^{2}, \theta\right)
$$

At leading order in $Q^{2}$ the reduced parity-violating asymmetry gives the proton's weak charge, $Q_{w}^{p}$. At next-to-leading order the $\mathrm{B}\left(Q^{2}, \theta\right)$ term contains infor- 
mation about the electromagnetic, weak, and strange form factors and is relatively suppressed at low $Q^{2}$. In choosing the momentum transfer at which the experiment was run this term was very important. Contributions from $\mathrm{B}\left(Q^{2}, \theta\right)$ can be reduced by lowering the $Q^{2}$, however this also reduces the magnitude of $\mathrm{A}_{e p}$, thus our ability to determine the asymmetry precisely. Setting the momentum transfer to 0.0025 $(\mathrm{GeV} / \mathrm{c})^{2}$ allowed for a precise measurement of $Q_{w}^{p}$ while constraining the contribution from $B\left(Q^{2}, \theta\right)$ to $\sim 30 \%$. The determination of $B\left(Q^{2}, \theta\right)$ was done using a global fit of the existing PVES data up to $0.63(\mathrm{GeV} / \mathrm{c})^{2}$. This fit as well as details of the extraction of $Q_{w}^{p}$ is discussed in Section 6.1.

\subsubsection{Precision Determination of $\sin ^{2} \theta_{w}$}
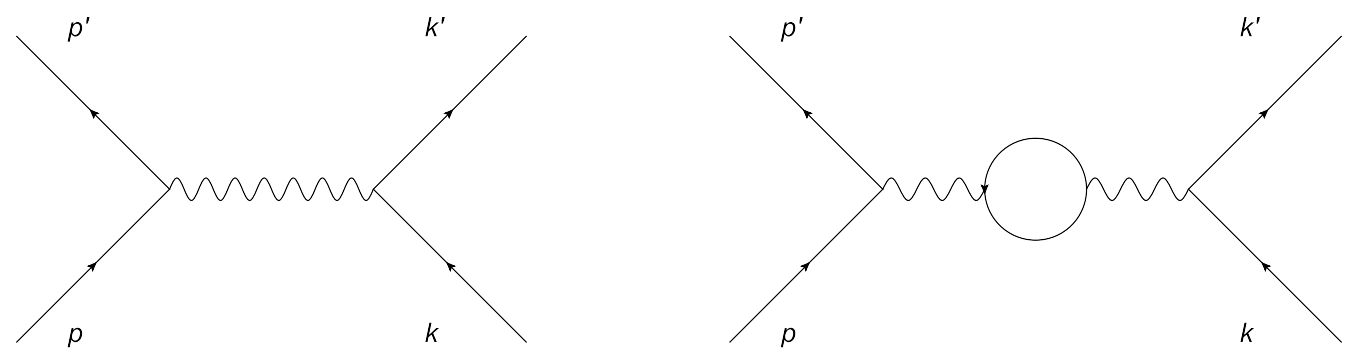

FIG. 2.2: The electromagnetic interaction at $\mathcal{O}(\alpha)$ (left) and $\mathcal{O}\left(\alpha^{2}\right)$ (right). The vacuum polarization screens the bare charge of the electromagnetic interaction at the vertex.

Thus far in this discussion, the primary focus has been on the leading order contribution to the physics asymmetry, however as either $Q^{2}$ increases or the precision of our measurement increases it is important to consider effects of higher-order contributions. Before discussing the implications of higher-order diagrams in the context of the weak-charge it is first instructive to look at the electromagnetic charge. To leading order the electromagnetic coupling is given by the fine-structure constant $\alpha$. Couplings, in general within the SM, are energy dependent and therefore as $Q^{2}$ in- 
creases higher-order diagrams, in powers of the coupling constant, must be included. The effect on the measured or "effective charge" can be thought of as an electron in a dielectric. This effect comes about because the vacuum acts as a dielectric; electron-positron pairs appear out of the vacuum, effectively screening the actual charge. The way in which the coupling changes as a function of $Q^{2}$ is called the running of the coupling.

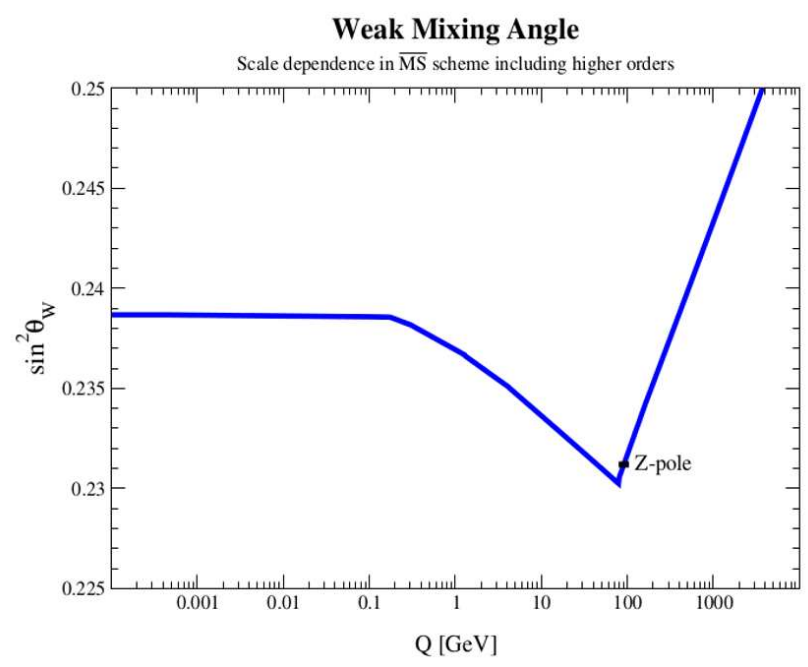

FIG. 2.3: The running of the weak mixing angle using the $\overline{M S}$ renormalization scheme[2]. The width of the curve represents the theoretical uncertainty in the calculation. The Z-pole value is given at $Q^{2}=M_{Z}$.

In the same manner as the electromagnetic coupling, the weak-coupling also has a dependence on $Q^{2}$. Calculations of the running of $\sin ^{2} \theta_{w}$ are included in $[2,38]$. The renormalization scheme was chosen to be $\overline{M S}[2]$ and is shown plotted as a function of $\mathrm{Q}$ in Fig. 2.3. Here the width of the line represents the theoretical uncertainty of the calculation. The large number of measurements taken at the Z-pole $\left(Q^{2}=M_{Z}\right)$ anchors the plot and measurements taken away from this $\mathrm{Q}$ provide important tests of the theoretical predictions of the SM. This places special importance on the understanding of the radiative corrections at the momentum 
transfer of $Q_{\text {weak }}$. The SM predicts a shift in $\sin ^{2} \theta_{w}$ from the Z-pole value at low momentum transfer of $\sim 0.007$. It is important to note that these corrections are renormalization scheme dependent. The weak-charge including radiative corrections can be written as [39]

$$
Q_{w}^{p}=\left[\rho_{N C}+\Delta_{e}\right]\left[1-4 \sin ^{2} \theta_{w}(0)+\Delta_{e^{\prime}}\right]+\square_{W W}+\square_{Z Z}+\square_{\gamma Z}
$$

where $\rho_{N C}$ accounts for one-loop corrections to the gauge boson propagators, which at tree level is defined to be $\rho_{N C} \equiv M_{W}^{2} / M_{Z}^{2} \cos ^{2} \theta_{w}$. The one-loop corrections come from the top and bottom quark loops to the gauge boson propagators; there are contributions from other quark generations but they are negligible. The terms $\Delta_{e}$ and $\Delta_{e^{\prime}}$ are the photon loop correction to the $\mathrm{Z}$ boson exchange vertex and the $\mathrm{Z}$ loop correction to the photon exchange vertex respectively. Diagrams for both $\rho_{N C}$ and $\Delta_{e, e^{\prime}}$ are shown in 2.4. The final three terms in 2.26 represent box diagrams
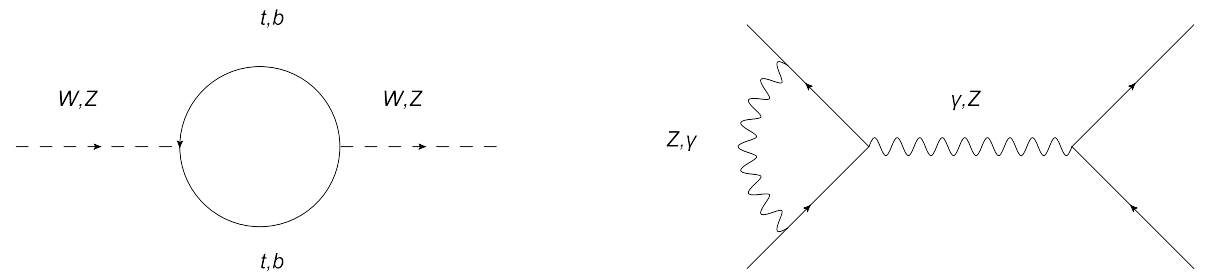

FIG. 2.4: The one loop contribution to $Q_{w}^{p}$ from the gauge boson mass renormalization is shown on the left. The $\gamma, \mathrm{Z}$ loop correction to the $\mathrm{Z}, \gamma$ exchange vertex is shown on the right.

describing the exchange of two gauge bosons. The box diagrams for $\square_{Z Z}$ and $\square_{W W}$ are relatively straight-forward to calculate using pQCD due to the propagators of the $\mathrm{W}$ and $\mathrm{Z}$ within the box being dominated by high momenta. The $\square_{\gamma Z}$ diagram is much more problematic because the photon is dominated by low momentum exchange which is outside of the useful regime of pQCD. Calculation of the $\square_{\gamma Z}$ diagram is discussed in more detail in 2.2.5. The box diagrams can be seen in Fig. 
2.5. Lastly, $\sin ^{2} \theta_{w}(0)$ is the one-loop definition of $\sin ^{2} \theta_{w}$ evaluated at $Q^{2}=0$.
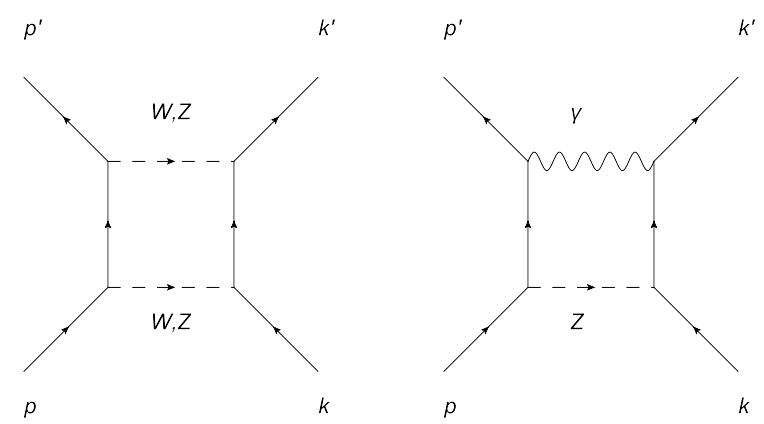

FIG. 2.5: Box diagrams representing the exchange of two gauge bosons (Cross terms not shown).

The one-loop definition of $\sin ^{2} \theta_{w}$ here contains contributions from mixing diagrams in which a Z-boson fluctuates into a photon via a fermion loop, single $\mathrm{W}$ loops, and two W loops containing goldstone bosons. A detailed derivation of the terms contributing to the running of $\sin ^{2} \theta_{w}$ can be found in $[2,40,38]$. Fig. 2.6 shows two examples of diagrams that contribute to the running.
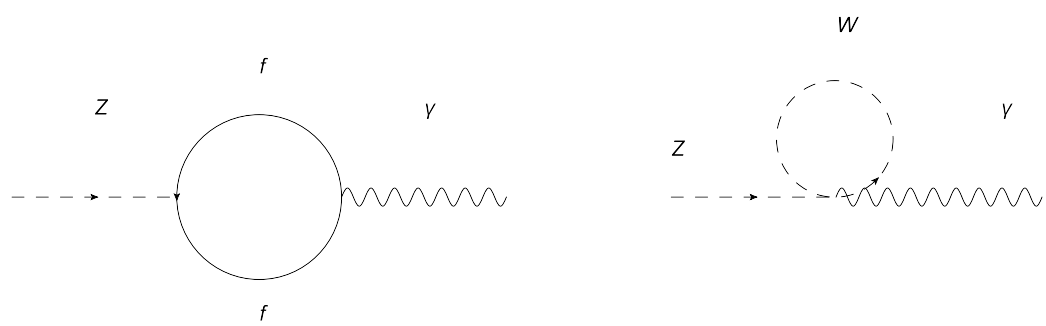

FIG. 2.6: Two examples of diagrams contributing to the running of $\sin ^{2} \theta_{w}$. The left diagram shows a $\mathrm{Z}$ boson fluctuating into a photon via fermion loop. The right diagram shows a single $\mathrm{W}$ loop.

\subsection{5 $\gamma Z^{0}$ Box Diagram}

In the forward-limit the dominant energy-dependent radiative correction to Eq. 4 comes from the $\gamma-Z$ box diagram $\left(\square_{\gamma Z}^{V}\left(E, Q^{2}\right)\right)$ which arises in the axial-vector coupling at the electron vertex. This correction has been evaluated using dispersion 
TABLE 2.2: Recent calculations of $\square_{\gamma Z}^{V}\left(E, Q^{2}\right)$ and its uncertainty at the kinematics of this measurement.

\begin{tabular}{lll}
\hline Reference & $\square_{\gamma \mathrm{Z}}^{V}\left(E, Q^{2}\right)$ & $\Delta \square_{\gamma \mathrm{Z}}^{V}\left(E, Q^{2}\right)$ \\
\hline Sibirtsev, et al. [41] & 0.0047 & ${ }_{-0.0004}^{+0.0011}$ \\
Rislow, et al. [40] & 0.0057 & 0.0009 \\
Gorchtein, et al. [42] & 0.0054 & 0.0020 \\
Hall, et al. [43] & 0.0056 & 0.00036 \\
\hline
\end{tabular}

relations $[41,40,42,43]$ independently by several groups, and is summarized in Table 1. The most recent calculation [43] of $\square_{\gamma \mathrm{Z}}^{V}\left(E, Q^{2}\right)$, uses parton distributions and benchmarking with recent $\vec{e} d \mathrm{PV}$ data at JLab[44] to reduce uncertainties. Their result corresponds to a contribution to the asymmetry at Qweak kinematics that is equivalent to a shift in the proton's weak charge of $7.8 \pm 0.5 \%$ of the tree-level SM value. 


\section{CHAPTER 3}

\section{Experimental Setup}

\subsection{Experimental Setup}

The experimental requirements associated with the $\mathrm{Q}_{w e a k}$ experiment brought about a unique set of technical challenges, due primarily to the proposed $2.1 \%$ statistical and $1.4 \%$ systematic uncertainty on the measurement of the part-per-billion level asymmetry. In an effort to meet these goals, the experiment built on technical advances made at Jefferson Lab, as well as expertise gained from previous precision PVES experiments [45, 46]. The design parameters (shown in Table 3.1) were chosen to optimize counting statistics and reduce systematics as much as possible. The primary limiting factor for the experiment was counting statistics, for this reason high current, highly polarized beam, and a high power target were required, as well as almost 2 years of non-continuous running. The major subsystems in the experiment include: a $2.5 \mathrm{~kW}$ cryotarget, lead collimation system, large acceptance Čerenkov detector array, toroidal spectrometer, rapid reversal polarized source, and tracking system used to measure the experimental $Q^{2}$. The experiment was run in two com- 


\begin{tabular}{|l|c|}
\hline Parameter & Value \\
\hline \hline Incident Beam Energy & $1.165 \mathrm{GeV}$ \\
Beam Polarization & $85 \%$ \\
Beam Current & $180 \mu \mathrm{A}$ \\
Target Thickness & $35 \mathrm{~cm}\left(0.04 \mathrm{X}_{\circ}\right)$ \\
Full Current Production Run Time & 2544 hours \\
Nominal Scattering Angle & $7.9^{\circ}$ \\
Scattering Angle Acceptance & $\pm 3^{\circ}$ \\
$\phi$ Acceptance & $49 \%$ of $2 \pi$ \\
Solid Angle & $37 \mathrm{msr}$ \\
Acceptance Averaged $Q^{2}$ & $0.026(\mathrm{GeV} / \mathrm{c})^{2}$ \\
Integrated Detector Rate (all detectors) & $6.5 \mathrm{GHz}$ \\
\hline
\end{tabular}

TABLE 3.1: Design Parameters for the $\mathrm{Q}_{w e a k}$ Experiment

plementary configurations: current-mode and tracking-mode. Current-mode, which is the default running for the asymmetry measurement, relies on high current (180 $\mu \mathrm{A}$ ) beam scattering from the $l \mathrm{H}_{2}$ cryotarget. Electrons scattered at a nominal angle of $7.9^{\circ}$ are focused into the radially symmetric detector array, using the toroidal spectrometer, where they are integrated at $960 \mathrm{~Hz}$ and read-out via 18-bit ADCs. In tracking-mode, which is run at low current $(\sim 1 \mathrm{nA})$, horizontal and vertical drift chambers are used to reconstruct the scattered electron track and measure the experimental $Q^{2}$ on the detectors. A number of ancillary measurements were also performed to quantify the contribution of background to the asymmetry measurement. In the following chapter a more detailed overview of the implementation and operation of the different subsystems is given. 


\subsection{Polarized Electron Beam}

\subsubsection{Continuous Electron Beam Accelerator Facility}

The Continuous Electron Beam Accelerator Facility (CEBAF) provides high quality polarized beam that is crucial to the PVES program at Jefferson Laboratory. The design of CEBAF provides an electron beam that, while having some time structure, is essentially continuous providing a duty factor of 100\% [47]. CEBAF consists of 2 parallel linacs comprised of 20 cryomodules which can produce $600 \mathrm{MeV}$ of acceleration using niobium Superconducting Radio Frequency (SRF) cavities. The use of SRF technology greatly reduces the power consumption by reducing ohmic heating that would be present otherwise. Each linac is attached to a total of 9 recirculating arcs; for each pass, electrons are selected using an RF separator into an arc with progressively stronger dipole magnets to accommodate the increased electron momentum while providing the same radius of curvature. At the exit of each arc an RF combiner recombines the beam and sends it back into the linac for acceleration. The beam is extracted using an RF separator, following the desired number of passes, at the exit of the south linac. The beam then enters the beam switch yard where is it sent to any of the 3 experimental halls. The race track design of CEBAF, shown in Figure 3.1, provides a total of 5 passes with a final maximum energy of $6 \mathrm{GeV}$ which can be delivered simultaneously to all three halls with a current in excess of $200 \mu \mathrm{A}$. 


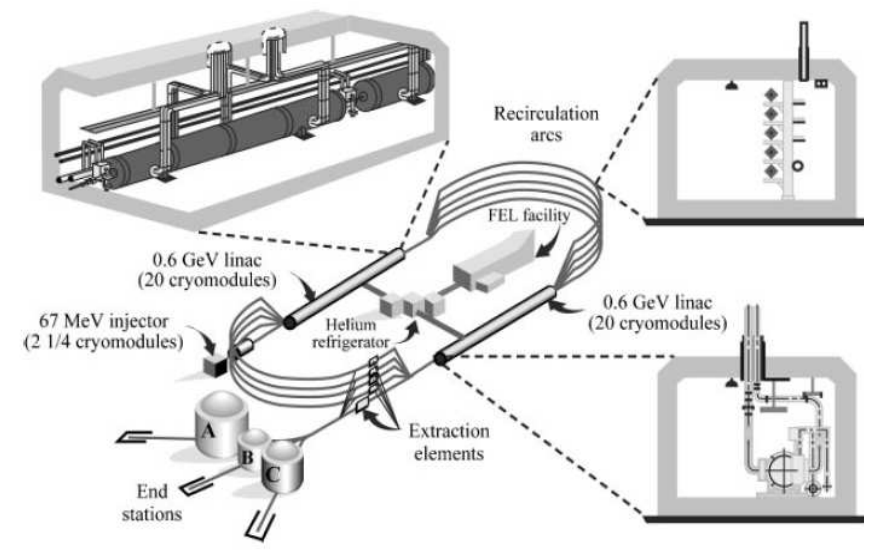

FIG. 3.1: CEBAF schematic.

\subsubsection{Polarized Source and Injector}

The production of spin-polarized electrons at Jefferson Laboratory begins in the injector where circularly polarized light incident on a photocathode of gallium arsenide (GaAs) is used to excite electrons from the conduction band to the vacuum. The electron affinity of GaAs, $\chi$, defined to be the energy difference between the vacuum level and the conduction band, is normally positive, however by treating GaAs with a layer of $\mathrm{Cs}_{2} \mathrm{O}, \chi$ can be reduced below zero giving a negative electron affinity (NEA)[3]. While a NEA photo-cathode is not a requirement of a spin-polarized electron source it significantly increases the emitted electron intensity and provides substantially better quantum efficiency (QE). The polarized electrons are produced using the process of optical pumping; when a photon with angular momentum $\sigma^{+(-)}$ is incident on a GaAs photo-cathode electrons transition from the conduction band substate $P_{3 / 2}$ to the valence band substate $S_{1 / 2}$. The transitions between $m_{j= \pm 3 / 2}$ and $m_{j= \pm 1 / 2}$ give a two-fold degeneracy which is illustrated in Figure 3.2. The fractional transition probability between the $P_{-\frac{3}{2},-\frac{1}{2}} \rightarrow S_{-\frac{1}{2}, \frac{1}{2}}$ and $P_{\frac{1}{2},-\frac{1}{2}} \rightarrow S_{\frac{1}{2}, \frac{1}{2}}$ 
shows photo-electrons are 3 times more likely to undergo the first transition than the second which limits the maximum theoretical polarization possible to $50 \%$.

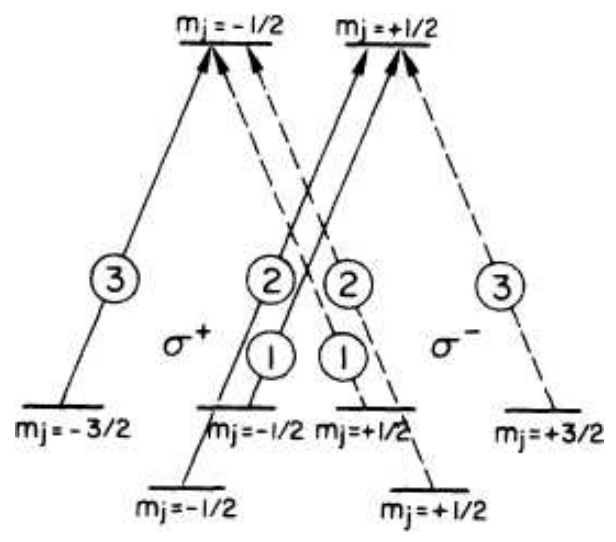

FIG. 3.2: The allowed optical transitions of $\Delta m_{j}= \pm 1$ in a GaAs photocathode are shown. The numbers in the circles represent the relative transition probabilities[3].

In order to raise the achievable polarization even further to accommodate ultraprecise parity experiments, it is desirable to break the degeneracy of the $P$ substate allowing a theoretical maximum polarization of 100\%. By growing the GaAs photocathode on a single layer of GaAsP it is possible to apply a mechanical strain to the photo-cathode breaking the degeneracy and pushing the polarization to $\simeq 75 \%$. Going even further, and alternating thin layers of GaAs/GaAsP, a super-lattice can be formed which can generate beam with a polarization of up to $90 \%$ and a significantly improved QE [48]. With this, the injector at Jefferson Laboratory routinely provides $85 \%$ polarized electron beam with a QE of near $1 \%$.

The circularly polarized light used to produce the electron beam in the injector is a product of the device chain shown in Figure 3.3. Three diode lasers are pulsed at $499 \mathrm{MHz}$ each, $120^{\circ}$ out of phase, with a combined bunch frequency of 1497 MHz. The resulting light is linearized using a linear polarizer, then passed through a Pockels cell which has a birefringence proportional to the electric field applied to the crystal medium. This birefringence rotates the polarization vector component 
differently depending on the axis, and therefore with the correct electric field the light becomes circularly polarized. By rapidly reversing the applied voltage it is possible to flip the polarization vector and thus reverse the direction of the electron polarization from the photo-cathode. Imperfections in the birefringent crystal can lead to residual linear polarization, which has the effect of adding a small amount of ellipticity to the polarized light. Most optical systems transport one linear polarization more effectively than others resulting in a helicity-dependent asymmetry in the amount of light delivered to the photo-cathode. This effect is traditionally referred to as the polarization-induced transport asymmetry (PITA)[49]. This effect is magnified by the fact that the GaAs photo-cathode has an analyzing power, i.e. emitted current from the photo-cathode is dependent on the orientation of the major-axis of the polarized light. This helicity-dependent effect generates a certain class of helicity-correlated beam asymmetries which include charge asymmetry.

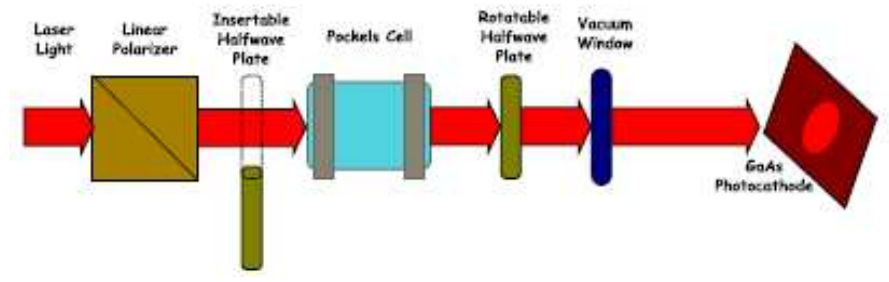

FIG. 3.3: Schematic of CEBAF injector optics setup[4].

The Rotatable Half-Wave Plate (RHWP) is used to minimize helicity-correlated asymmetries by rotating the residual linear polarization vector. This is done by matching up the major-axis of the exiting laser light to the axis of the analysing power such that the residual linear polarization is equal in magnitude in both polarization states. The Insertable Half-Wave Plate(IHWP) flips the sign of the linearly polarized light striking the Pockels Cell and therefore changes the sign of the circular polarization leaving the Pockels Cell. This is an important slow reversal because 
it cancels any systematics such as beam steering and electronic cross-talk which do not change sign with the IHWP[4].

Polarized electrons extracted from the photo-cathode are accelerated into the injector via the $100 \mathrm{kV}$ DC gun, which is enclosed in an Ultra High Vacuum $\left(10^{-11}\right.$ $10^{-12}$ Torr). This reduces the effects of back-scattered ions in the enclosure colliding with the photo-cathode which would degrade the QE. With this, the photo-cathode can provide an integrated charge of approximately $500 \mathrm{C}$ before there is a need to restore the photo-cathode yield. Electrons accelerated into the injector are longitudinally polarized parallel(antiparallel) to the beam momentum, however during transport through the linacs and arcs the electrons exhibit an in-plane precession which may result in a net transverse polarization at the experimental hall. This effect can be removed using a Wien Filter which rotates the electron spin in the injector such that it cancels the net spin procession in the accelerator $[50,51]$. A Wien Filter consists of an electric and magnetic field perpendicular to one another and perpendicular to the beam momentum. Originally used as a velocity selector, charged particles with a velocity satisfying $\beta=|E / B|$ are passed without deflection but with their spin vector rotated. Building on this, the Two Wien Filter Spin Flipper at CEBAF shown in Figure 3.4 uses a Vertical Wien Filter to rotate the spin $90^{\circ}$ to the vertical, a pair of solenoids to rotate transversely into the horizontal plane, and a Horizontal Wien Filter to completely reverse the initial electron spin. This forms the basis for an entirely new method of slow reversal which allows the electron spin to be flipped independently of the laser. 


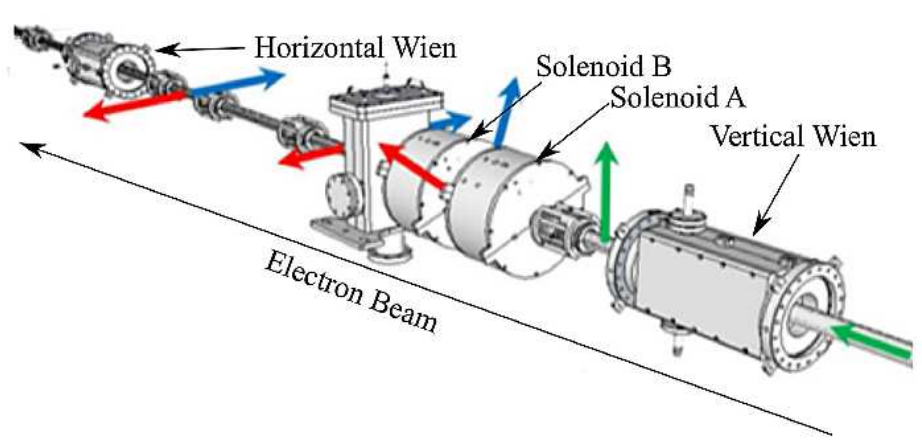

FIG. 3.4: The Wien filter causes a spin precession of the electron spin. The double Wien filter uses a pair of Wiens, Vertical and Horizontal, to orient the electron spin so as to cancel out transverse polarization due to spin procession in the linac and arcs. This allows $100 \%$ longitudinal polarization to be delivered to the experimental halls [5].

\subsubsection{Beam Position Monitors}

Beam transport information in the injector and the Hall $\mathrm{C}$ line is provided by beam position monitors (BPM), which play an important role in providing quality electron beam at Jefferson laboratory. BPM's are cylindrical cavities containing 4 symmetrical stripline wires rotated to $45^{\circ}$ in the right-handed Hall $\mathrm{C}$ coordinate system, which couple resonantly $(1497 \mathrm{MHz})$ to the RF-signal of the passing beam, providing signal amplitudes proportional to the proximity of the beam to the signal wires. The rotation of the signal wires is needed to alleviate the effects of synchrotron radiation in the horizontal plane. The BPM readout electronics can be adjusted to handle a range of currents $(1 \mu A-200 \mu A)$ by adjusting the gain settings on the pickup wires. Each of these signals is transmitted to the BPM readout electronics where it is amplified and down-converted to $1 \mathrm{MHz}$ before being digitized and read out via custom $Q_{\text {weak }}$ ADC's at $960 \mathrm{~Hz}$ in integrating mode. Figure 3.5 shows a cross-section of a BPM cavity and stripline wires. 


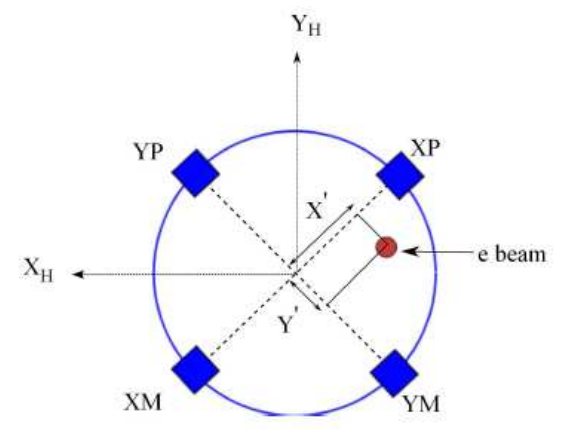

FIG. 3.5: Schematic of cylindrical BPM as seen along the beam pipe[6]. Signal wires are shown rotated in the counter-clockwise direction to $45^{\circ}$. Hall coordinates are given by $\mathrm{X}_{H}$ and $\mathrm{Y}_{H}$.

The relative beam position in the unrotated basis can be written in terms of the raw signals in the form of the asymmetry between wires along a given rotated axis. In order to properly calculate the absolute beam position two important factors need to be addressed[52].

- The amplitude gains may be different between channels.

- The pedestals in each channel are not zero and must be accounted for.

In the unrotated basis the absolute beam position in $\mathrm{X}^{\prime}$ can be written in the form:

$$
X^{\prime}=\kappa \frac{\left(X P-X P_{\text {offset }}\right)-\alpha_{X}\left(X M-X M_{\text {offset }}\right)}{\left(X M-X M_{\text {offset }}\right)+\alpha_{X}\left(X P-X P-{ }_{\text {offset }}\right)}
$$

Here $\alpha$ is the ratio of the gains between two channels along a given axis, and $\kappa$ is the sensitivity of the BPM to the $1497 \mathrm{MHz}$ RF-signal, which converts the readout signal to $\mathrm{mm}$. The $\mathrm{Y}^{\prime}$ position can be calculated in a similar manner. The pedestals are defined as the BPM readout at zero current however, due to the non-linear behaviour of the BPM's at low current, they must be measured in a region of linear operation and calculated using extrapolation. BPM calibrations are done at high current (nominally $180 \mu A$ ) in fixed gain mode by varying the beam current $\pm 10-15 \%$ 
and measuring the monitor response. A linear fit to the monitor response allows extrapolation to zero current and robust determination of the pedestal value[6]. The absolute beam positions read out by the data acquisition system are rotated into the accelerator coordinate system,

$$
\begin{aligned}
& X=\frac{1}{\sqrt{2}}\left(X^{\prime}-Y^{\prime}-X_{\text {offset }}^{\prime}\right) \\
& Y=\frac{1}{\sqrt{2}}\left(X^{\prime}+Y^{\prime}-Y_{\text {offset }}^{\prime}\right)
\end{aligned}
$$

where the offset variables shift the origin of the relative beam position into the origin of the accelerator coordinate system.

The final BPM in the Hall C line is located $1.4 \mathrm{~m}$ upstream of the $Q_{\text {weak }}$ target making direct measurement of the electron beam position at the target impossible. In order to determine the beam position(angle) on target, virtual BPMs are constructed using the $5 \mathrm{BPMs}$ in the drift region to project to the target. Virtual BPMs are constructed using a linear least-squares fit to the absolute BPM positions. A similar measurement of the relative energy at the target can be made by considering the computed position(angle) at the target and the horizontal beam position at the point of highest dispersion in the Hall $\mathrm{C}$ arc as measured by BPM 3C12X. The functional form of the energy variable, qwk_energy, is given by:

$$
\frac{d P_{\text {target }}}{P_{\text {target }}}=\frac{X_{3 C 12 X}-M_{11} X_{\text {target }}-M_{12} X_{\text {target }}^{\prime}}{M_{15}}
$$

where $M_{i, j}$ are elements of the transport matrix which map position, angle, and relative energy at the target to the corresponding measurements at the point of highest dispersion in the Hall $\mathrm{C}$ arc. Here we have only considered elements of the transport matrix which contribute significantly to the determination of qwk_energy. 
A list of the BPM's used in the construction of the virtual BPM's can be found in Table 3.1.

\begin{tabular}{|l|l|}
\hline Run Period & BPMs used in Virtual BPM \\
\hline Beginning of running to run 14486 & $3 \mathrm{H} 07 \mathrm{a}, 3 \mathrm{H} 07 \mathrm{~b}, 3 \mathrm{H} 07 \mathrm{c}, 3 \mathrm{H} 09,3 \mathrm{H} 09 \mathrm{~b}$ \\
\hline Run 14486 to end of running & $3 \mathrm{H} 07 \mathrm{a}, 3 \mathrm{H} 07 \mathrm{~b}, 3 \mathrm{H} 07 \mathrm{c}, 3 \mathrm{H} 09$ \\
\hline
\end{tabular}

TABLE 3.2: Hall C BPMs used to construct virtual target BPMs. BPM 3H09b was removed due to functionality issues in the second half of running.

\subsubsection{Beam Current Monitors}

The electron beam current in CEBAF is monitored using beam current monitors $(\mathrm{BCM})$ in the injector and the end of the accelerator. Measuring the beam current in two locations helps to monitor and limit beam loss through the machine, and provide the most accurate measurement of the beam incident on the target. Current measurements made on the accelerator side were done using a pair of BCM cavities in conjunction with an Unser monitor. The BCM's are cylindrical RF cavities resonant at the fundamental frequency of the beam $(1497 \mathrm{MHz})$. As the beam passes through the $\mathrm{BCM}$ it excites the transverse electric mode, $\mathrm{TE}_{010}$ via a large loop antenna coaxial with the cavity resulting in a DC voltage level linearly proportional to the beam current. The BCMs are temperature controlled to reduce the effects of heating due to power dissipation in the cavity. The high-frequency output signal is downconverted from $1497 \mathrm{MHz}$ to $1 \mathrm{MHz}$. The BCM signals are then read out into the DAQ using $\mathrm{Q}_{\text {weak }}$ ADCs. Table 3.2 shows the 6 BCM cavities active during different run periods over the full experiment. For purposes of noise reduction, a charge variable (qwk_charge) was formed using the average of the most robust current monitors during each run period. 


\begin{tabular}{|l|l|l|}
\hline Run Period & Active BCM & qwk_charge \\
\hline Run 1 & BCM1, BCM2, BCM5, BCM6 & BCM1, BCM2 \\
\hline Run 2 & BCM5, BCM6, BCM7, BCM8 & BCM7, BCM8 \\
\hline
\end{tabular}

TABLE 3.3: Beam Current Monitors activity periods. The monitors used to form the qwk_charge variable are listed. BCMs $1-2$ were used primarily during Run I while BCMs $5-8$ were being commissioned. BCMs $5-8$ were built with lower noise digital receivers.

The BCM cavities provide a relative current measurement and therefore must be properly calibrated in order to make an accurate measurement. This done using an Unser monitor. The Unser monitor is a parametric DC current transformer which provides a non-invasive beam current measurement[53]. The Unser monitor contains a small toroid sensor which develops a magnetic field as the beam passes through it. The DC component of the magnetic field is detected and cascaded through a feedback loop which generates a current in the secondary windings exactly canceling the magnetic flux of primary windings. The current seen in the feedback loop is then proportional to the detected beam current.

\subsection{Electron Beam Polarimetry}

Precise measurement of the longitudinal beam polarization is crucial to the measurement of $Q_{w e a k}^{p}$. The uncertainty in the beam polarization measurement represents the dominant relative systematic uncertainty contribution expected to the physics asymmetry $(\delta \mathrm{P} / \mathrm{P}=1 \%)$. The beam polarization was measured using two devices: The existing Hall C Møller polarimeter which provides an absolute invasive polarization measurement at beam currents of $\sim 1 \mu \mathrm{A}$, and the newly constructed 
Hall C Compton polarimeter which provides a high current, non-invasive, continuous measurement of the beam polarization.

\subsubsection{Møller Polarimeter}

The Møller polarimeter relies on cross-section asymmetry $e^{-} e^{-} \rightarrow e^{-} e^{-}$scattering for precise determination of the beam polarization. Møller scattering is a pure Quantum Electrodynamic (QED) process so the cross-section can be calculated very precisely. In the center-of-mass (CM) frame the scattering cross-section for Møller scattering at lowest order can be written as [54]:

$$
\frac{d \sigma}{d \Omega}=\frac{d \sigma_{o}}{d \Omega}\left[1+P_{t}^{\|} P_{b}^{\|} A(\theta)\right]
$$

where $d \sigma_{o} / d \Omega$ is the unpolarized cross-section, $P_{t}^{\|}$is the target polarization, $P_{b}^{\|}$ is the beam polarization, and $A(\theta)$ is the analyzing power. Considering the crosssection asymmetry,

$$
\frac{d \sigma_{o}^{\uparrow \uparrow} / d \Omega-d \sigma_{o}^{\uparrow \downarrow} / d \Omega}{d \sigma_{o}^{\uparrow \uparrow} / d \Omega+d \sigma_{o}^{\uparrow \downarrow} / d \Omega}=A(\theta) P_{t}^{\|} P_{b}^{\|},
$$

the beam polarization can be obtained with knowledge of the analyzing power and polarization of the target. The analyzing power is determined using simulation and is maximized by detecting scattered and recoiled Møller electrons at $90^{\circ}$. The Hall C Møller polarimeter, shown in Figure 3.6, scatters longitudinally-polarized electrons from a $1 \mu \mathrm{m}$ thick iron foil target. The iron foil target is polarized to saturation along the beam axis using a $3.5 \mathrm{~T}$ superconducting solenoid field. At saturation, the electron polarization is known precisely[55]. Scattered electrons are horizontally focused using quadrupole magnet Q1 and the desired scattering angles are selected using the collimator system, after which they are again horizontally defocused using 
quadrupole Q2. The scattered electrons are then detected in coincidence using two symmetrically-placed lead-glass detectors. Detecting the scattered electrons in coincidence greatly reduces contamination from Mott scattering and other backgrounds. This allows measurement of the electron beam polarization with a statistical error

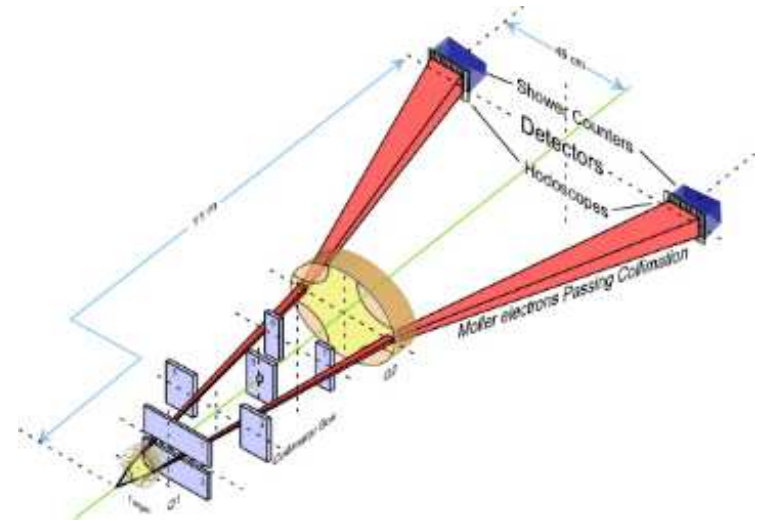

FIG. 3.6: Schematic for Hall C Møller Polarimeter[7].

of $\sim 0.5 \%$ in 5 minutes. Because the Møller measurement requires the insertion of a special target into the beam path, and is therefore invasive to normal $\mathrm{Q}_{\text {weak }}$ running, Møller measurements were performed only periodically.

\subsubsection{Compton Polarimeter}

The Hall C Compton polarimeter provides continuous, non-invasive measurement of the electron beam via $e^{-} \gamma \rightarrow e^{-} \gamma$ scattering. The detection of both the back-scattered electrons and the photons provides two important, semi-independent measurements of the electron beam polarization. The cross-section asymmetry for Compton scattering is given by,

$$
A(k)=P_{\gamma} P_{e} A_{z}(k),
$$


where $A(k)$ is the cross-section asymmetry, $P_{\gamma}$ is the circular polarization of the laser, $A_{z}(k)$ is the analyzing power, and $P_{e}$ is the electron beam polarization. The Compton polarimeter, which was designed and commissioned primarily for $\mathrm{Q}_{\text {weak }}$, consists of a magnetic chicane, laser system, a photon and electron detector, and a laser monitoring system. The layout of the Hall C Compton polarimeter can be seen in Figure 3.7. The incoming electron beam is directed into the Compton chicane

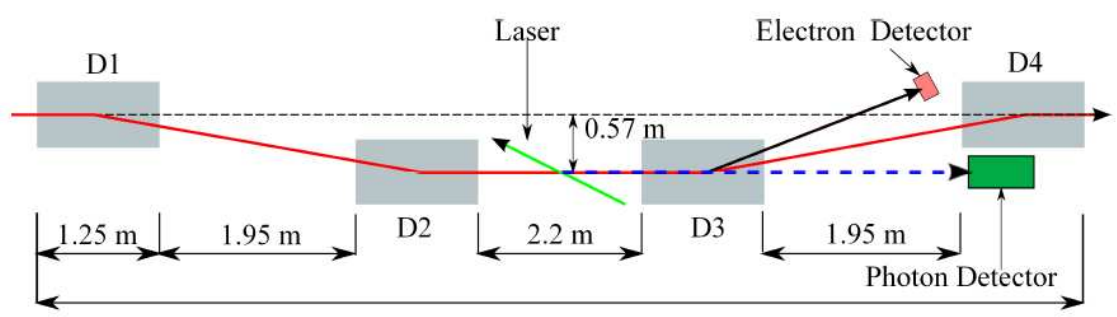

FIG. 3.7: The Hall C Compton layout. D1-4 are dipole magnets, the electron beam is shown in red, black denoted the scattered electrons, and the dashed blue shows the scattered photons.

using dipoles D1-2 where it interacts with a photon target provided by a low-gain Fabry-Perot cavity coupled to a 10 Watt continuous-wave green laser $(532 \mathrm{~nm})$. The scattered electron are then deflected into the electron detector while the scattered photons, which are emitted in a cone around the scattered electrons, are detected in the photon detector which is located along the chicane axis. Unscattered beam is directed out of the Compton chicane and back into the main beamline using dipoles D3-4. The array of 4 dipole magnets in the Compton chicane are aligned in a symmetric arrangement and have the benefit of deflecting the beam with no net spin procession. Due to the Compton polarimeter being in commissioning it was not available until April 2011, and is not considered in this analysis. 


\subsection{Primary $l \mathrm{H}_{2}$ Target}

The primary scattering target for $\mathrm{Q}_{w e a k}$ was a $34.4 \mathrm{~cm}$ long conical liquid hydrogen cell designed to sustain $180 \mu \mathrm{A}$ of beam current, while keeping noise due to target boiling $<5 \%$ of the width if the physics asymmetry distribution. This corresponds to a target noise contribution of $<50$ ppm based on the $5.3 \mathrm{GHz}$ per detector scattering rate in the experiment. Target heating, due to the incident electron beam, causes density fluctuations in the target, which can induce noise into the measured main detector asymmetry. In order to reduce systematic effects due to target heating, which can be a significant source of noise, the electron beam was rastered in a $3.5 \times 3.5 \mathrm{~mm}^{2}$ uniform distribution. The target length was chosen to yield more scattering events, while the conical shape accommodates the optimal scattering angle of $7.9^{\circ}$. The $l \mathrm{H}_{2}$ target was operated at a pressure of 35 psi and a temperature of $20.00 \pm 0.02 \mathrm{~K}$. The liquid volume of the target was approximately 55 liters when fully condensed. The target cell in which the incoming electron beam interacts can be seen in Figure 3.8a and includes an entrance and exit window composed of Al 7075-T6 which was chosen for its especially high tensile strength. The target windows, which generate a significant amount of background, were made as thin as possible. The entrance window has a uniform thickness of $0.096 \mathrm{~mm}$, while the exit window consists of two regions: an outer diameter which has a thickness $0.635 \mathrm{~mm}$ and an inner region of diameter $15 \mathrm{~mm}$ and a thickness of $0.125 \mathrm{~mm}$. The unscattered beam passes through the inner region, which is thinner to reduce backgrounds. Elastically scattered electrons pass through the experimental acceptance $\left(6^{\circ}<\theta<12^{\circ}\right)$ and exit the target through the thicker outer diameter of the target window. The focus of scattered electrons, passing through the outer region of the exit window, is affected due to radiative losses. Characterization and measurement 


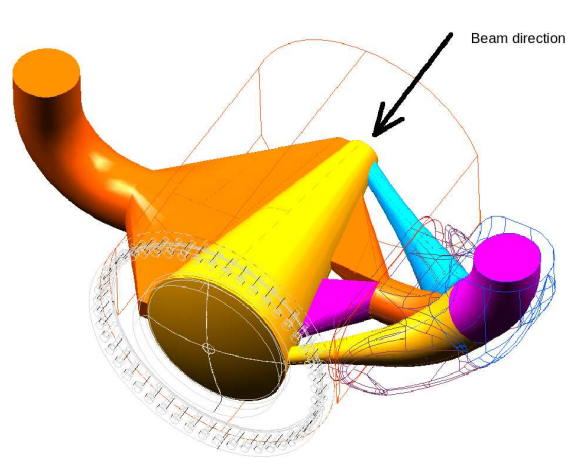

(a) Target cell drawing.

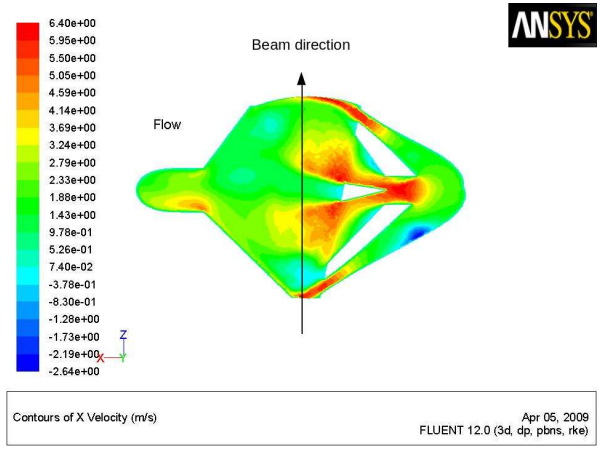

(b) CFD simulation of target flow velocities.

FIG. 3.8: (a) CAD drawing of the conical target cell. The conical shape of the cell accommodates the out going scattered electrons. (b)CFD simulation showing $\mathrm{lH}_{2}$ flow velocities inside the target cell[8].

of these radiative losses was the subject of target window studies[8]. One of the primary concerns of high current beam on a $l \mathrm{H}_{2}$ target is heating; $1.165 \mathrm{GeV}$ electrons passing through the target experience an ionizing energy loss of $\sim 2.1 \mathrm{~kW}$, which is deposited as heat in the target. Additional contributions include: viscous heating, pump heat, as well as conductive and radiative heat loss. Including $150 \mathrm{~W}$ of reserve for the feedback loop, the total cooling power needed by the target is $2.5 \mathrm{~kW}[56]$. To meet the cooling requirements of the $Q_{\text {weak }}$ target a large amount of effort was put into the unique design of the target cell using Computational Fluid Dynamics (CFD) simulations. The simulations effort help to optimize the circulation of the $l \mathrm{H}_{2}$ in the target cell minimizing heating on the target windows and reduce hot spots that would otherwise lead to target boiling. Examples of target flow velocity profiles from CFD can be seen in Figure 3.8b. The $l \mathrm{H}_{2}$ in the target cell is recirculated at $1.2 \mathrm{~kg} / \mathrm{s}$ transverse flow, using a $746 \mathrm{~W}$ centrifugal pump. A 3kW hybrid heat exchanger, designed by the Jefferson Lab cryo group, was employed to help meet target cooling demands. The heat exchanger combines two source of cooling, a $4 \mathrm{~K}$ supply from the Central Helium Liquefier, and a 15K supply from the End Station 
Refrigerator, into a single unit. In addition to this, a high-power heater is used to stabilize the target temperature when the beam is not present. The allowed contribution to the systematic error of physics asymmetry, from target density fluctuation due to target heating, was $<50 \mathrm{ppm}$ or $\sim 5 \%$ of the quartet level asymmetry width. In order to measure the actual error contribution, a number of systematic studies looking at dependence on raster size and $l \mathrm{H}_{2}$ pump frequency were performed. The total contribution to the experimental asymmetry width due to target boiling was found to be $46 \mathrm{ppm}$.

\subsection{Infrastructure}

\subsubsection{Collimation System}

A collimator system was used in the experiment to reduce the effects of inelastics and secondary particles in the detectors, and optimize the uncertainty in the measurement. The system consisted of three $15 \mathrm{~cm}$ thick lead collimators, each with eight $400 \mathrm{~cm}^{2}$ apertures placed symmetrically around the beamline (see Figure 3.9). The primary and tertiary collimators were designed to protect the tracking system and the magnetic spectrometer from intense gamma radiation and inelastics coming from the scattered beam. The secondary collimator (defining collimator) defines the solid-angle acceptance for the experiment to be $4 \%$ of $\pi$ in the azimuth and $49 \%$ of $2 \pi$ in the polar angle. Although the primary function of the collimators is to define the experimental acceptance and optimize the uncertainty of the measurement of $\mathrm{Q}_{\text {weak }}^{p}$, GEANT simulations found that the inner edge of the defining collimator which has direct line of sight to the detectors - was a potential source of background in the form of high energy photons. This background, estimated to be on the order 
of $1 \%$, led to the design and installation of a set of lintels, which acted as an effective fourth collimator, to block line of sight neutral backgrounds.

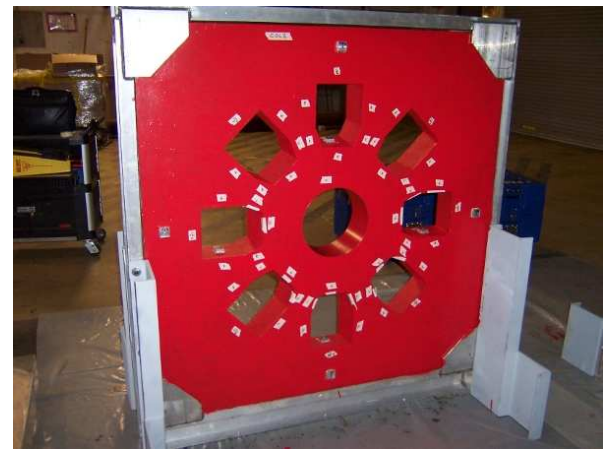

FIG. 3.9: The defining collimator shown before installation. This collimator defines the experimental acceptance for $Q_{w e a k}[8]$.

\subsection{2 $\mathrm{Q}_{\text {weak }}$ Toroidal Spectrometer}

Particles within the specified acceptance exit the collimator and enter the $\mathrm{Q}_{\text {weak }}$ toroidal (QTOR) spectrometer where charged particles are focused azimuthally towards the detectors. QTOR is specifically optimized to focus elastically scattered electrons, at the $\mathrm{Q}_{\text {weak }}$ beam energy and angle, onto the detectors; inelastics and Møller electrons, which have a lower energy are swept away. Positively charged particles are bent inward and absorbed by the thick shielding surrounding the beamline. The QTOR spectrometer (Figure 3.10a) provides a toroidal magnetic field of $\sim 0.9$ T-m and consists of eight water cooled coils, situated symmetrically around the beamline, held in place by an aluminium support structure making it completely iron free. The initial field mapping of the magnet was done at MIT-Bates after which the magnet was disassembled, transported to JLab, and reassembled. After installation in the experimental hall at JLab the magnet was remapped and calibrated to ensure precision installation. The reassembly was found to be within \pm 5 $\mathrm{mm}$ of that found at MIT-Bates [57]. 


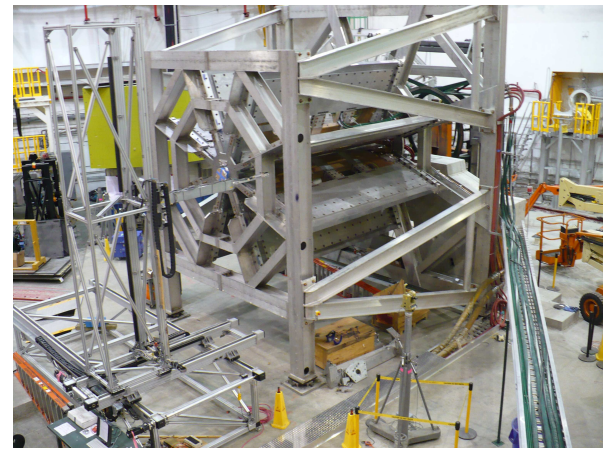

(a) QTOR during installation.

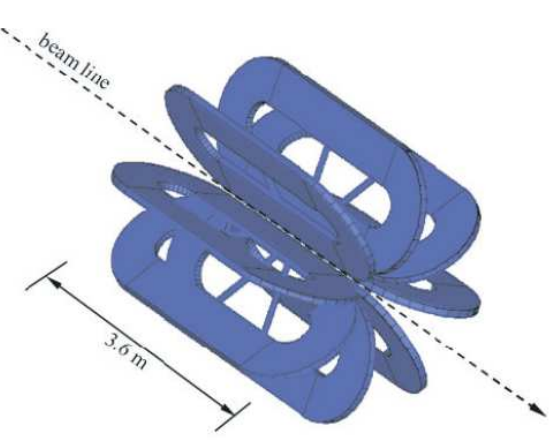

(b) QTOR Diagram.

FIG. 3.10: (a)Qweak Toroidal Spectrometer installed in experimental Hall C at Jefferson Lab. (b) Current in each coil travels in a racetrack fashion creating a toroidal magnetic field. The field goes to zero at the center allowing the beam to pass unperturbed to the beam dump.

\subsubsection{Shielding Wall}

An $80 \mathrm{~cm}$ thick concrete shielding wall (Figure 3.11) was placed downstream of QTOR, preceding the detector array. The goal of the shielding wall was to provide an aperture which would reduce backgrounds resulting from secondaries and inelastics interacting with structural supports in QTOR. GEANT 3 Simulations showed that in the region of the shielding wall there was an overlap between the elastic and inelastic signal; great care was taken in the design of the shielding wall apertures to assure that, while blocking backgrounds from the spectrometer region, backgrounds resulting from interactions of the inelastic signal with the inner edge of the aperture were as small as possible[9].

\subsubsection{Quartz Čerenkov Detectors}

The main detectors for the experiment were responsible for measuring the rate of elastically scattered electrons in each helicity state, which is used to measure the PV asymmetry. Each main detector had dimensions of $200 \mathrm{~cm} \mathrm{x} 18 \mathrm{~cm} \mathrm{x} 1.25 \mathrm{~cm}$ 


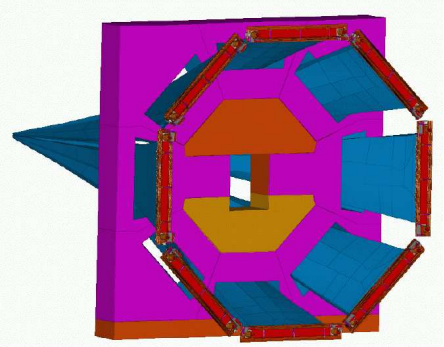

FIG. 3.11: CAD diagram of shielding wall. Simulated beam envelope shown in blue. Proper design of the shielding wall was crucial to avoid interaction of the scattered envelope with the inner edge of the apertures[9].

and was composed of Spectrosil 2000 synthetic fused-silica bars. Each detector bar was a composite of a pair of $100 \mathrm{~cm}$ pieces of fused silica glued together with UVtransparent glue (SES-406). The Spectrosil 2000 synthetic fused-silca was chosen for the detector material on the merits of its radiation hardness, low sensitivity to neutral background, and uniform response across the detector. Each detector bar was placed in a light tight box and supported by an aluminium support structure. The "ferris wheel"support structure held one detector package at a radius of 335 $\mathrm{cm}$ in each of the 8 octants $\left(0^{\circ}, \pm 45^{\circ}, \pm 90^{\circ}, \pm 135^{\circ}, 180^{\circ}\right)$. A diagram showing the azimuthal placement of the main detector packages is shown in Fig. 3.12. Scattered electrons leave the collimator apertures and travel into the main detectors; electrons moving faster than the phase velocity of light in the quartz medium generate Čerenkov radiation. The Čerenkov light is internally reflected down the bar, through a light guide, and into photo-multiplier tubes (PMT) mounted on each end. Each light guide was a simple extension of the main quartz bar with the PMTs attached to the ends using optical glue. The 5" PMTs gathered light using UV-transparent glass and operated in two modes depending on the PMT base used: low-gain mode (gain $\mathcal{O}\left(10^{3}\right)$ ) which was used for high current integrating mode, and high-gain (gain $\left.\mathcal{O}\left(10^{6}\right)\right)$ mode which was used primarily for tracking mode. Signals produced in the 


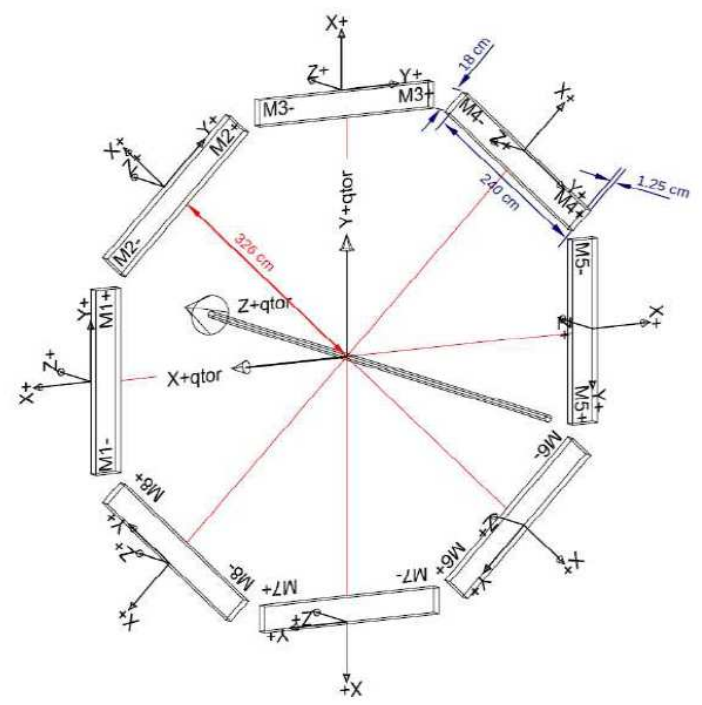

FIG. 3.12: Schematic of quartz detector array.

PMTs were read out using low-noise electronics designed and constructed at TRIUMF. A current-to-voltage preamp converts the current produced in the PMT to an analog pulse. It is then integrated and recorded via an 18-bit ADC operating at $500 \mathrm{~Hz}$. Bench tests of the low-noise electronics showed a noise contribution to the asymmetry width of $7 \mathrm{ppm}$.

One of the primary concerns mentioned in previous sections has been the contribution of soft backgrounds to our detector signal. Simulation indicated that significant backgrounds would be present during high current running - photon rates due to bremsstrahlung were predicted to be $\mathcal{O}(200 \mathrm{MHz})[58]$. Photons of energy less than $\mathcal{O}(100 \mathrm{keV})$ should not produce a signal in the detectors[59], however at higher energies Compton scattering and pair production can occur inside the quartz and electron-positron pairs can produce a background signal. In order to reduce the effect of these backgrounds in the quartz detectors, $2 \mathrm{~cm}$ thick lead pre-radiators were added to the main detectors. This has the effect of reducing backgrounds by absorption of soft photons and increasing the signal-to-noise ratio from electrons in 
the elastic envelope showering inside the pre-radiator. The pre-radiator did however induce a level of noise in the detectors $(\sim 12 \%)$ due to light-yield fluctuations in the number of electrons produced in the shower; this increased the amount of run time need by 370 hours but was out weighed by the reduction in the relative background versus not using the pre-radiators.

\subsubsection{Beamline Shielding}

Scattered electrons leaving the scattering chamber at small angles $\left(0.75^{\circ}-4^{\circ}\right)$ and passing through the first clean-up collimator can potentially interact with the downstream beam pipe causing a significant source of background - primarily in the form of neutrals[8]. While efforts were made to design the $\mathrm{Q}_{\text {weak }}$ beamline to accommodate this, simulations showed significant background generation. A tungsten beam plug was installed in the downstream side of the first collimator to block these events. The plug was water-cooled to dissipate $\sim 1.3 \mathrm{~kW}$ of expected heating due to the scattered beam being dumped into the plug. Figure 3.13 shows a simulation of the beamline background with and without the tungsten plug; it is apparent from the simulation results that the addition of the tungsten plug significantly reduced both the neutral and multiple-scattered electron contributions to the beamline background. The tungsten plug, however, becomes a source of secondary photons in the collimator region with potential to re-scatter and generate background in the detectors. Measurements to determine how to best reduce backgrounds coming from the beamline were performed during the commissioning phase, and led to the installation of a number of lead bricks around the beamline as well as a 12" long, 2" thick lead donut around the beam pipe in the collimator region. 

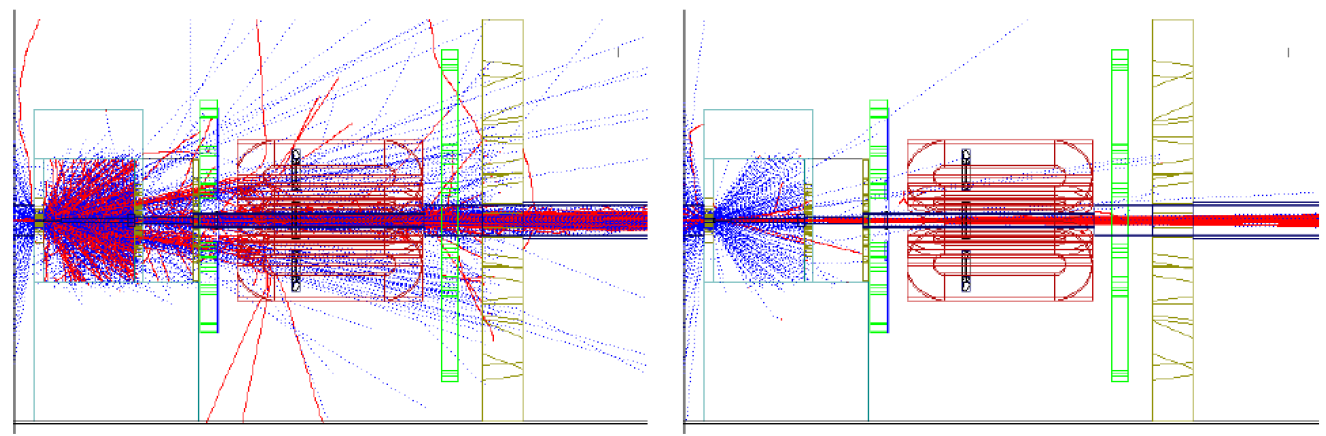

FIG. 3.13: Simulated beamline background with(right) and without(left) the tungsten plug. Electrons are shown in red and neutral are shown in blue. The addition of the tungsten plug drastically reduces the beamline backgrounds[8].

\subsubsection{Beam Modulation System}

Changes in the beam properties during helicity flip can lead to false asymmetries in our detected scattering rates. The scattering rate in the detectors depends significantly on five parameters: transverse position, angle, and incident energy E. Small helicity-correlated variations in these parameters produce false asymmetries which are potentially enhanced by various broken symmetries in the experimental apparatus. The experiment took a two-pronged approach to measurement of detector sensitivity to changes in the beam position, angle, and energy. The first method was to measure the correlation of the measured asymmetry in the detectors with the helicity-correlated difference of the natural beam jitter seen in the beam monitors and to use standard multivariate linear regression techniques to extract the decoupled detector sensitivities. The second - and a major topic of the work found in this thesis - was to modulate the beam parameters in a controlled way using an external driving signal and extract the sensitivities from the observed detector response. In order to achieve this, a beam modulation system was designed and installed for the $\mathrm{Q}_{\text {weak }}$ experiment. The details of the design, implementation, and analysis efforts using this method are detailed in the following chapter. 


\subsection{Tracking System}

The parity-violating asymmetry, at $\mathrm{Q}_{w e a k}$ kinematics, can be expressed in powers of $Q^{2}$ (see eq. 2.25); therefore, precise determination of $Q^{2}$ is essential to the extraction of the weak charge. Specifically, the acceptance-weighted distribution of $Q^{2}$, weighted by the detector response needs to be determined to $\sim 1 \%$. This is important because the non-uniformity of light collection in the detector affects the measured $Q^{2}$ at the percent level. The goal of the tracking system was to measure the acceptance-weighted $Q^{2}$, and use it for a benchmark simulations which can be used to calculate $Q^{2}$ at the scattering vertex. As a secondary motivation, the tracking system can be used to study various backgrounds including scattering from the target windows that may contribute to the measured asymmetry. The tracking

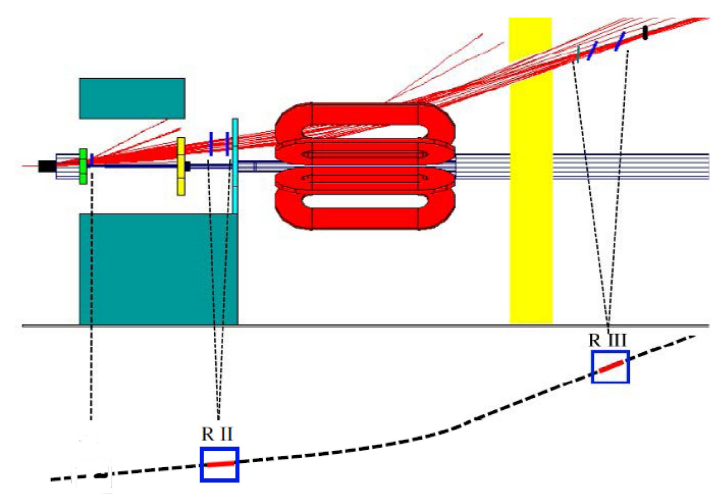

FIG. 3.14: Simple schematic of the experiment showing the 3 tracking regions. Simulated trajectories of elastically scattered electrons are shown in red

system consists of two regions: Region II, and III and was operated at low currents (50 pA - 50nA) due to limitations on the rates that the detectors in Regions II and III could handle. The low rates allowed the detectors to be run in counting-mode, meaning that individual detected particle could be recorded and used in track reconstruction. Region II contained a set of horizontal drift chambers (HDCs), which 
were used to determine the scattering angle and initial particle trajectory. Region III consisted of a pair of vertical drift chambers (VDCs), which provided particle position and angle information after the magnetic field. Attached to each pair of VDCs was a trigger scintillator, which provided the tracking event trigger. Detector packages in Region II and III were mounted in opposing octants on rotatable support structures which allowed for a complete mapping of the main detector acceptance. Also, the rotating support structures allowed the tracking detectors to be moved radially out of the beam during high current running. A simple schematic of the three tracking regions is shown in Figure 3.14.

\subsubsection{Horizontal Drift Chambers}

The Region II HDCs are located just upstream of the last collimator and provide position and angle information about the scattered electrons entering the magnetic spectrometer. The HDCs were designed to have a position resolution of $\sim 200 \mu \mathrm{m}$ and an angular resolution of $\sim 0.6 \mathrm{mrad}$. The active area of the HDCs was $38 \mathrm{~cm} \times$ $28 \mathrm{~cm}$ and each HDC unit contained 6 wire planes $\left(u, v, x, u^{\prime}, v^{\prime}, x^{\prime}\right)$. Each wire plane contained 192 sense wires; wires in planes $\left(x, x^{\prime}\right)$ were strung horizontally, wires in planes $\left(u, u^{\prime}\right)$ were angled by $53.2^{\circ}$, and wires in planes $\left(v, v^{\prime}\right)$ were angled by $-53.3^{\circ}$. Each wire plane was situated between two aluminized Mylar plates held at -2150 V, while the sense wires were grounded. Each chamber was sealed air-tight and filled with a $65 \%-35 \%$ argon- ethane mixture; the argon/ethane mixture is stable and is easily ionized. The gas mixture was bubbled through isopropyl alcohol to reduced the effects of chamber ageing. A total of 5 chambers were built with the intent of using 4 chambers in the experiment with the $5^{\text {th }}$ as a back-up. Each HDC "package" consisted of two chambers mounted with a $40 \mathrm{~cm}$ offset along the beam 


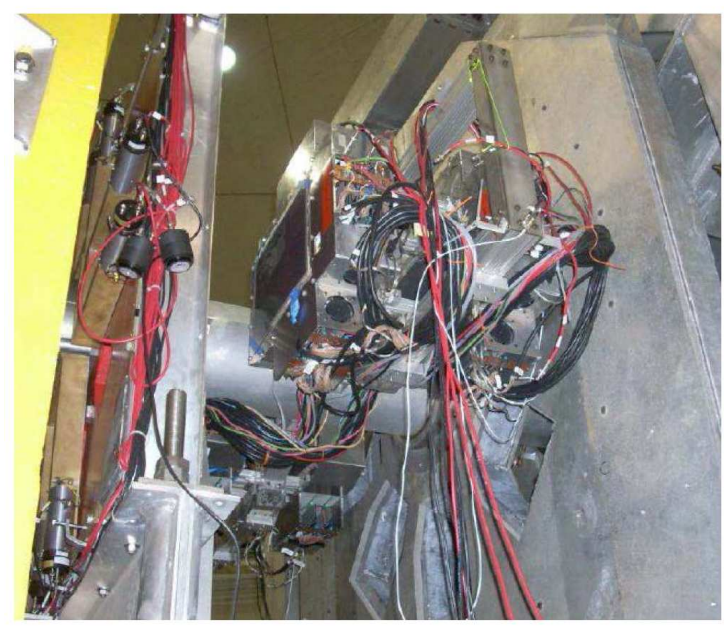

FIG. 3.15: Region II HDCs installed downstream of QTOR in Hall C.

axis. The HDC packages were mounted in opposite octants of a human-powered rotating support structure, the region II "rotator", which allowed the HDCs to be used to map the full acceptance. The region II rotator also allowed the HDCs to be retracted radially inward - out of the scattered electron envelope - during highcurrent running. Charged particles passing through the HDCs ionize the gas; ionized electrons drift towards the sense wires. Drift electrons nearing the sense wire begin an avalanche increasing the total charge - and therefore the signal size - seen when the wires are read out. A photo of the region II HDCs installed in Hall C can be seen in Fig. 3.15.

\subsubsection{Vertical Drift Chambers}

The VDC's, located downstream of the QTOR and the shielding wall, served to provide information about the electron's position and angle after leaving the magnetic field of QTOR. Each drift chamber had an active area of $0.914 \mathrm{~m} \mathrm{x} 2.438$ $\mathrm{m}$ and contained two wire planes, labeled $u$ and $v$, each with 279 wires. The wires in each plane were strung at an angle of $26.45^{\circ}$, which was found to be the optimal 
angle using the simulation packge Garfield[10, 60], and with a spacing of $11.12 \mathrm{~mm}$. A full detector package consisted of 2 wire planes, 3 Mylar foils, 2 gas planes, and one spacer frame (see Fig. 3.16b). Each frame was made from G10-FR4, which is a sandwich of glass fibers and epoxy chosen for its ability to withstand compression, strong dielectric properties, and minimal gas absorption. These properties were important because each chamber was filled with a 50\%- 50\% argon- ethane gas mixture and had to be air-tight. The Mylar cathode planes were held at $-3800 \mathrm{~V}$ and each sense wire in planes $u$ and $v$ was held at ground.

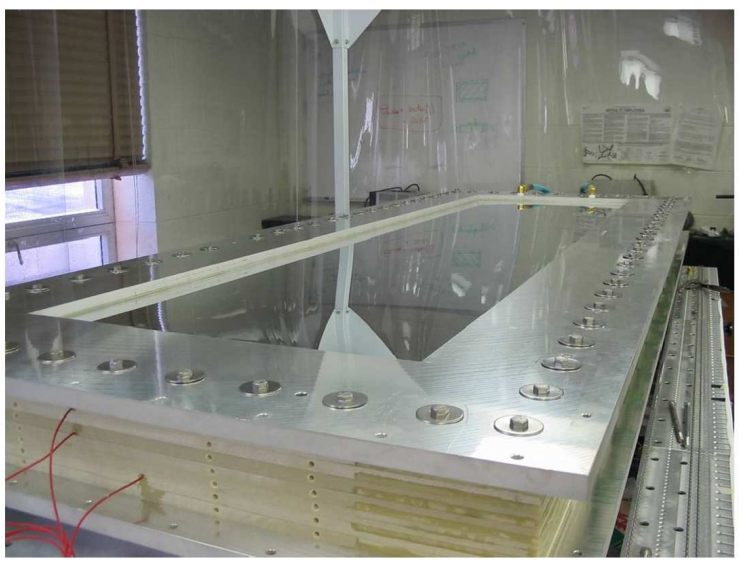

(a) Vertical Drift Chamber

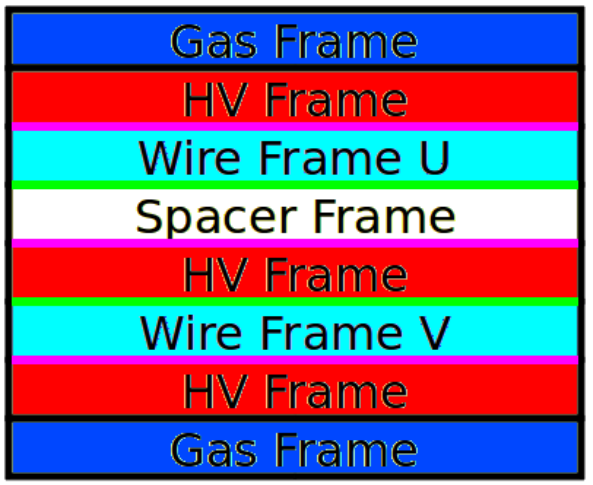

(b) Layering for one VDC. The HV foils are shown in magenta and wire planes are shown in green. The center foil was aluminized foil on both sides to provide field in both cells of the VDC.

FIG. 3.16: (a) Shows one completed vertical drift chamber in the lab at College of William and Mary. The VDCs were layered (b) and contained 2 wire planes, $3 \mathrm{HV}$ planes, a spacer frame, and 2 gas frames[10].

Each VDC pair was mounted on a large rotatable support structure, the Region III "rotator" (the topic of the next section), in opposing octants. As with the HDCs, the VDC pairs could be rotated to all octant pairs, allowing for full coverage of the azimuthal acceptance, as well as being retractable, which allowed them to be moved out of the scattered envelope during high current running. The chambers are 
mounted at a $24.4^{\circ}$ angle with respect to the support structure mounts and therefore have a smaller angular acceptance than the HDCs but a higher spatial resolution $(<200 \mu m)$.

Signals in the VDC are produced in much the same way as with the HDCs; scattered electrons entering the VDC at an angle and ionize the gas in each VDC's "cell". The free electrons in the gas are then accelerated along the electric field lines towards the ground wires; as the free electrons drift they ionize more of the gas, creating more free electrons, cumulating in an avalanche when reaching the wires. For a single VDC package there are a total of 558 individual channels to read out or 2232 channels for the entire Region III tracking system. The read-out of such a large number of channels posed a significant logistical and cost challenge for the experiment. In response to this issue the detector signals were multiplexed, which reduced the number of channels required by a factor of 9 .

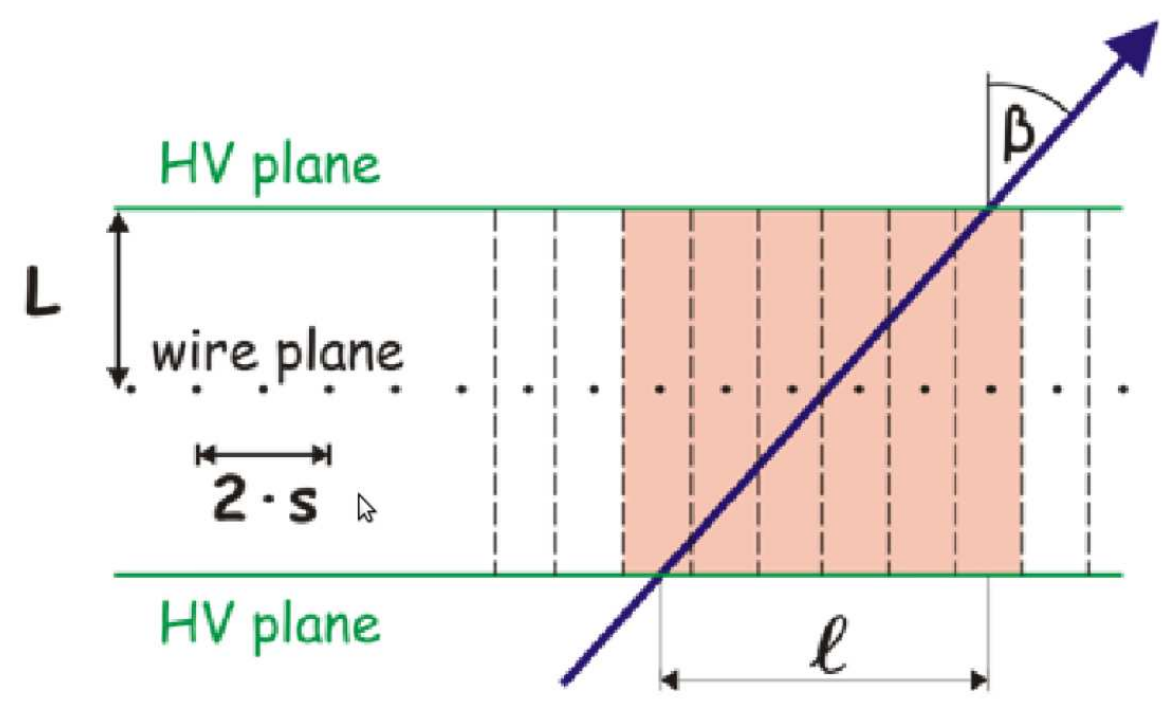

FIG. 3.17: Cross-section of one VDC "cell". HV planes are shown in green and the particle track is shown in blue[10]. Particles entering the cell ionize the gas causing an avalanche of charge moving towards the signal wires. The timing of these signals is used to extract track information. 


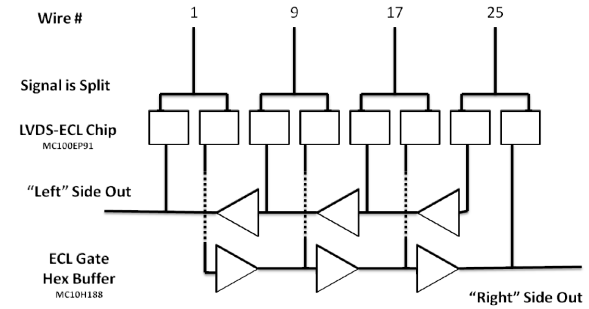

(a) Diagram of LVDS-ECL signal multiplexing in the MUX crates.

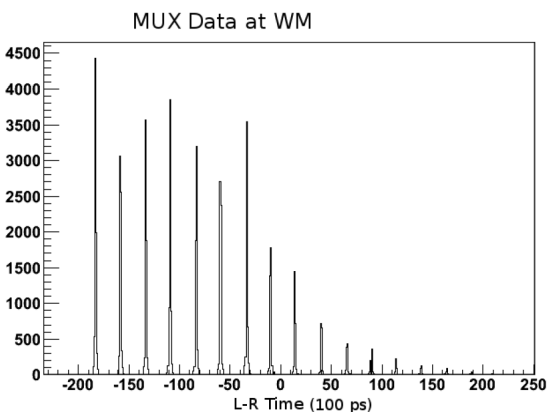

(b) Time difference data from bench testing.

FIG. 3.18: (a)Diagram of LVDS-ECL read-out and signal multiplexing in the MUX crates. A single delay line is shown for the first four multiplexed signals. (b)Typical time difference data from bench testing of the MUX crates. Each peak represents a wire in the delay chain; peak separation is $\sim 1.3$ ns with an average $\sigma$ of $80 \mathrm{ps}[10]$.

Each wire signal was read out using a custom pre-amp/discriminator board which amplified the analog signal and converted it to a low-voltage differential logic signal (LVDS). The LVDS signal is then converted to an emitter-coupled logic (ECL) signal and fed into a custom JLab 64-channel time-to-digital converter (TDC) which recorded the arrival time of the incoming logic signal. The signal multiplexing took place in custom built multiplexing (MUX) crates containing LVDS-ECL conversion boards. The multiplexing was done by combining the signal from every $9^{\text {th }}$ wire into a single read-out channel with a small delay provided by the ECL gate/buffer chips. An example of the signal read-out can be seen in Fig. 3.18a. Each signal was split and processed by the LVDS-ECL chip; signals were then read out on both the "left" and "right" sides.

Depending where along the signal chain the signal is it will encounter a different number of delay buffers (each delay is $\sim 1.3 \mathrm{~ns}$ ). The signals were processed by subtracting the times for the left and right signals, which produced a well defined peak for each wire, as seen in Fig. 3.18b. 


\subsubsection{Trigger Scintillators}

The trigger scintillators served to provide a fast timing trigger for trackingmode data and were located $40 \mathrm{~cm}$ upstream of the main detectors. Each trigger scintillator was held in place using aluminum supports attached to the Region III VDCs. Each scintillator bar was $218.45 \mathrm{~cm} \mathrm{x} 30.48 \mathrm{~cm} \mathrm{x} 1.00 \mathrm{~cm}$ thick and was made from Bicron BC-408 plastic scintillator which is sensitive to charged particles and insensitive to neutrals. The scintillator bars had light guides made from UVT lucite stranded and attached to each end; the UVT lucite strand was choosen over a triangular light guide due to a factor of 2 increase in their light collection and $30 \%$ increase in timing resolution[8]. On the end of each light guide was attached a

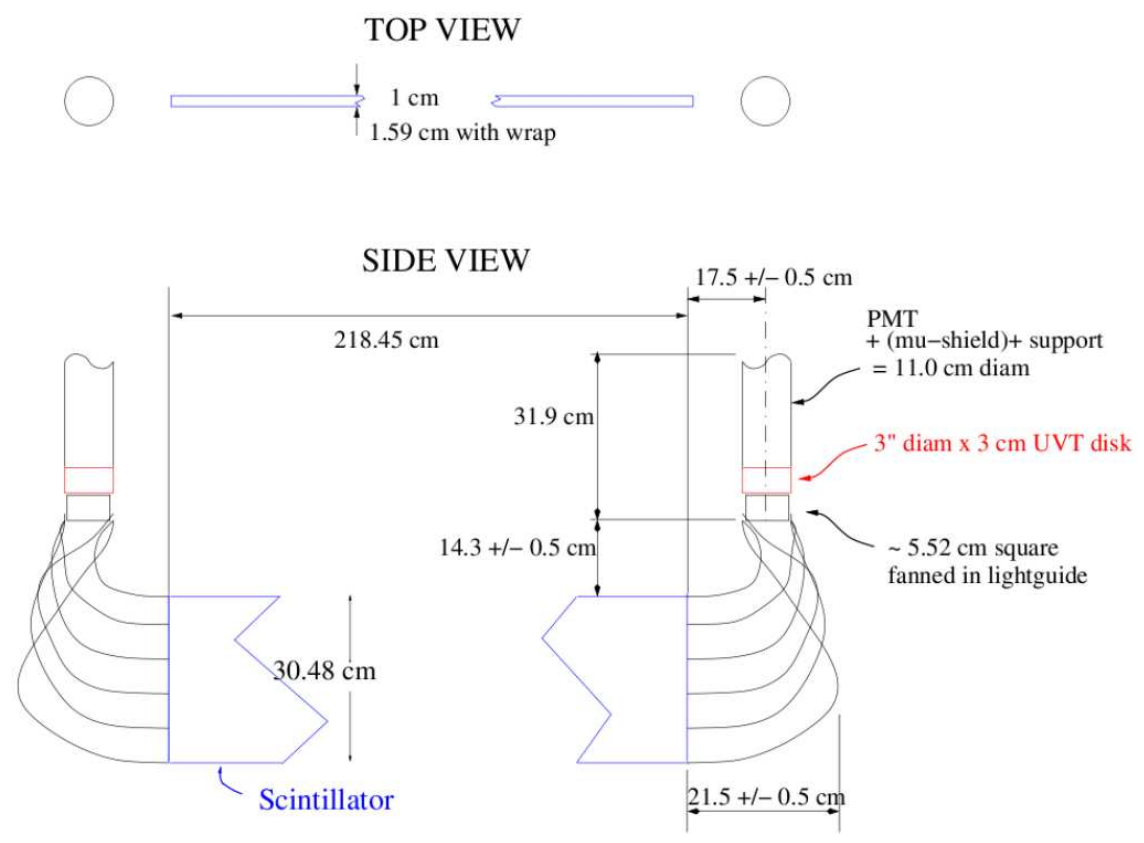

FIG. 3.19: Schematic of trigger scintillator including dimensions[8].

Photonis XP-4312B 3" high gain $\left(\sim 3 \times 10^{7}\right)$ PMT which converted the scintillator light into an electrical output signal. The signals produced by each PMT were combined using a CAEN V706 16-Channel Mean-time Module, which produces an 
output signal independent of the hit position along the length of the scintillator bar. This eliminates the time difference between signals seen in PMTs at each end of the trigger scintillators - and provides a constant timing signal - in the case a hit is closer to one side than the other.

\subsection{Rotator}

As mentioned previously, both the Region II and Region III tracking detectors had access to the full azimuthal acceptance through rotation of their support structures. One stark difference between Region II and Region III was that while the Region II HDCs - each HDC weighed $\sim 25 \mathrm{~kg}$ - were easily rotated by hand, the Region III VDC package (VDCs plus mounting plates) weighted $\sim 953 \mathrm{~kg}$ and were rotated around a horizontal axis $3.96 \mathrm{~m}$ vertically from the hall floor and at a radius of $2.3 \mathrm{~m}$. In this case, manual rotation was not a viable option and a mechanically driven rotation system was designed. The Region III rotator was designed to provide a semi-automated method of rotating the Region III VDCs through the full azimuthal acceptance, to provide radial motion that can move and retract the VDCs during high current running, and to provide sufficient reproducibility given the resolution of the VDCs. The system included radial and rotational motion systems, detector cabling management, position tracking, an air-driven position locking system, and a safety system. Each of these systems, their design criteria, and measures of their performance will be discussed at length in the following subsections. More detailed specifications will be listed in the appendix. 


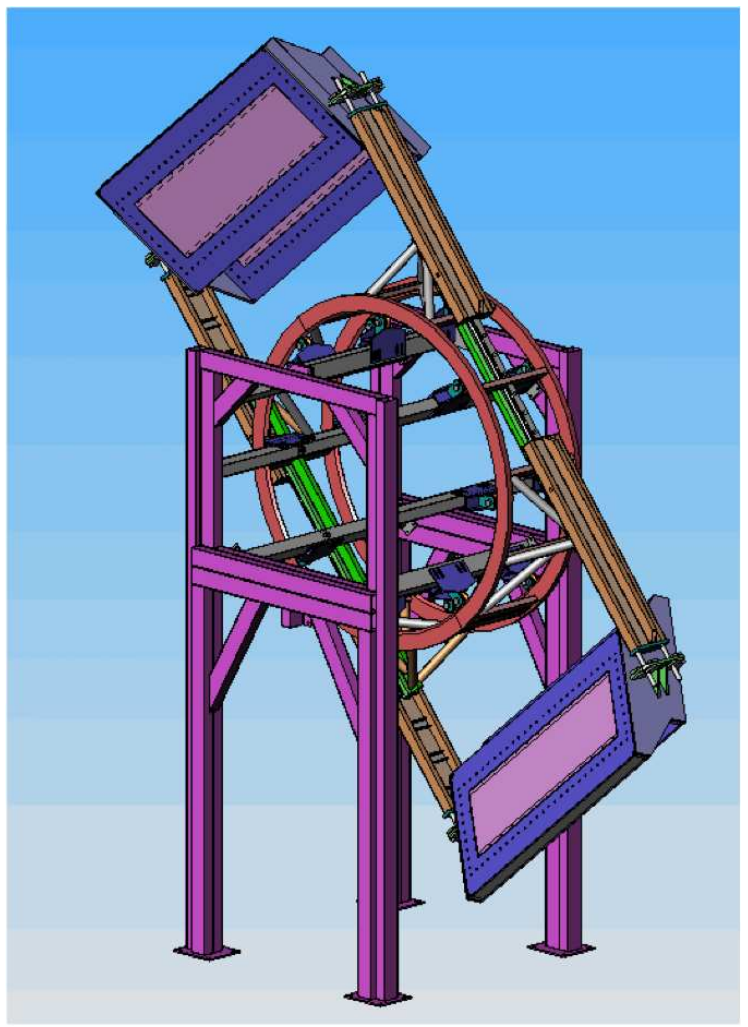

FIG. 3.20: Region III rotator.

\subsubsection{Structural}

The Region III rotator (see Fig. 3.20), at its center, consists of a $2.3 \mathrm{~m}$ radius 304 stainless steel hub which is held concentric to the beamline. The 304 stainless steel, which makes up the structure of the rotator, was chosen for its low magnetic permeability ${ }^{1}$. The central rotator hub is held in place via lateral struts attached to a simple steel support base. Each lateral strut has two 5-inch V-groove rollers attached to a base allowing them to be adjusted radially and in $\mathrm{Z}$ along the beam direction. The inner edge of the central hub is beveled to fit the $\mathrm{V}$-groove cams. During installation the rotator hub was situated on the adjustable rollers, and a survey was used to adjust the centroid of the rotator hub to be centered on the beamline. The

\footnotetext{
${ }^{1}$ The low magnetic permeability material was chosen to reduce effects due to polarization of the support structure caused by the near-by QTOR field and polarized electron scattering.
} 
pitch was also adjusted to eliminate any tilt in rotator face with respect to the plane perpendicular to the beam direction. Two parallel $2.88 \mathrm{~m}$ rails are mounted on the
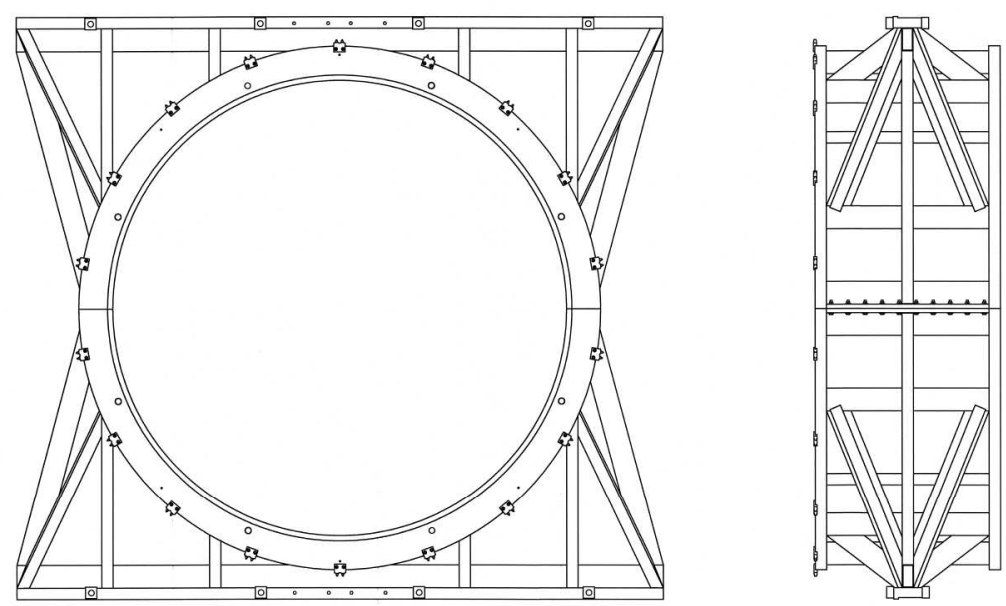

FIG. 3.21: The central hub of the Region III rotator. The rotator support hub was machined from 304 stainless steel; this material was chosen because of it low magnetic permeability. Steel rails, which the rotator arms ride on, were attached to the flat structures protruding from the central hub.

central hub. The rotator arms, designed and assembled by Jefferson Lab, can be seen in Fig. 3.22a. Each arm had two $35.56 \mathrm{~cm}$ threaded rods attached to the ends on which the VDC packages were attached. The rotator arms were attached to the central hub via four linear motion bearing blocks. These bearing blocks allowed the arms to slide along the mounting rail during retraction(extension) of the VDC packages. The arms were moved along the rails using a set of linear stepper motors mounted to the slider supports. The linear motion system is discussed in more detail in Sect. 3.7.2. The full detector assembly consisted of the two mated steel 'Z' plates to which the VDCs were bolted. The Z-plates held the VDCs offset from each other in the Y-axis and at an angle with respect to the plane of the rotator arms. The full detector assembly was mounted on the arms using the $35.56 \mathrm{~cm}$ long threaded rods; the mounting bracket on the Z-plates, to which the threaded rods 
were attached, were adjustable in $(X, Y, Z)$. This allowed the chamber's positions to be finely adjusted to the proper location with respect to the collimator apertures.

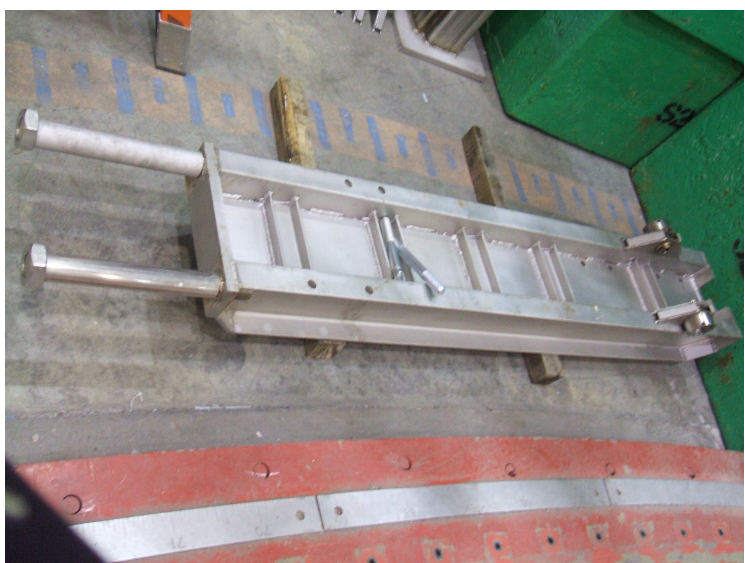

(a) The sliding arms can be seen in the Hall $\mathrm{C}$ before being installed. The threaded rods which were used to hold the VDC packages were covered with steel tubes prior to installation. The mounting brackets for the pancake cylinders can be seen in the back right.

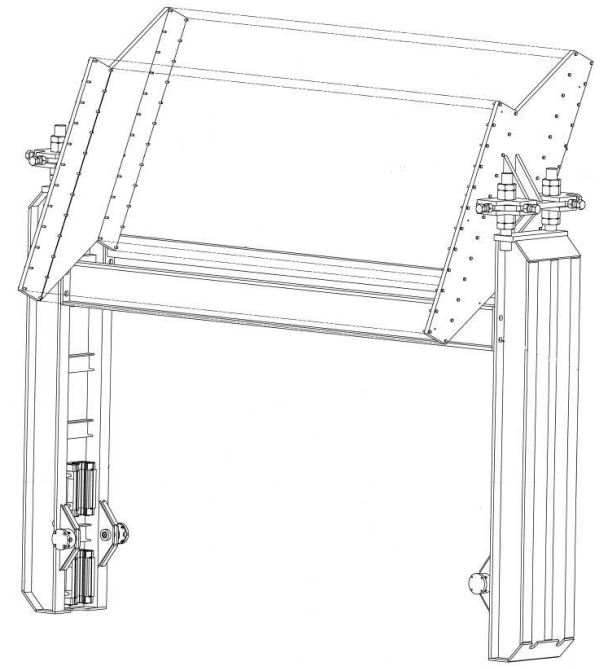

(b) CAD diagram of the fully assembled VDC packages mounted between the slider arms.

\subsubsection{Linear Motion System}

The Region III detectors were only used during tracking measurements and certain focused ancillary measurements; the chambers were designed to be operational at charged particle rates $\mathcal{O}(k H z)$ per octant and were retracted during high current running which had rates $\mathcal{O}(G H z)$. The linear motion of the VDC detector packages was achieved using a linear motion system which relied on two linear stepper motors (EC35005B ) from IDC Motion. When choosing a stepper motor two things were taken in to consideration: the linear motors needed to have the force required to move the chambers and the position resolution in line with what was needed to determine the track position in the VDCs. To make the linear motion safer and easier the decision was made to only operate the linear motion system while the chambers 
were in the horizontal position; this reduced the load on the motors to a minimum. The required thrust load needed was estimated assuming a coefficient of friction between the arms and the rails of 0.5 ; this corresponds to steel on steel. This is of course an over estimation, but gave us sufficient overhead so that we would easily be able to move the chambers. The estimated weight of the VDC detector package (two VDCs plus support plates) was $9346 \mathrm{~N}$ which would give a required linear thrust of $4673 \mathrm{~N}$; the maximum thrust specification on the linear stepper motors was $7200 \mathrm{~N}$ which is well above what was required. The position resolution on the motors was rated to be $0.039 \mu \mathrm{m} /$ step with a repeatability of $13 \mu \mathrm{m}$, which was well below the $\sim 200 \mu \mathrm{m}$ VDC position resolution. Each linear motor was driven using a stand alone motor controller (S6961). The motors were controlled via a RS232 communication port on a single board computer located in the controls crate. The motor controllers were wired in series so that the serial communication commands would be sent to both controllers at the same time, thus ensuring the motors would not become out of phase (see figure 3.23). While the rotator hub was locked in place using a positioning pin during linear motion, an unbalanced load due to the motors not being in sync was not desirable. The controls system will be discussed in more detail in Sect. 3.7.4. The linear motors were mounted using a specially designed motor mount to the central hub over the sliding rail to which the arms were attached. The linear motor shafts were attached to the arms using stainless steel eyelets welded to the posterior side of the sliding arms; each motor shaft had a clevis mount at the end which could be attached to the eyelet on the arms. When the rotator arms were extended they were held in place using $2.5 \mathrm{~cm}$ diameter locking pins. Each pin - two per arm located on opposite sides - was driven into a pin block mounted on support structure below the sliding rails. Each pin block was stainless steel with a brass insert in the center. The brass insert was added due to problems 


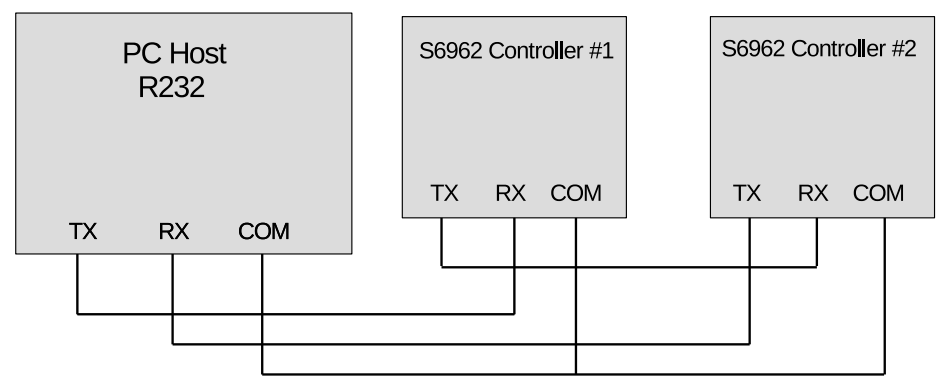

FIG. 3.23: Schematic showing the communications diagram for the linear motor controls.

with actuating the locking pins. When the locking pins were inserted and the arms were rotated into a position, where a substantial amount of the VDC weight was placed on them, pressure welding would take place making it extremely difficult to remove them. Adding the brass insert to the pin block provided a buffer between the steel pin and the pin block material.

\subsubsection{Rotational Motion System}

The rotational motor system provided $\pm 180^{\circ}$ rotational freedom to the VDCs; this provided full azimuthal coverage of the detectors in each octant pair. The rotational motor system is made up of a simple chain-drive powered by a $3 \mathrm{hp} \mathrm{AC}$ motor. For the rotational motor an AC motor was chosen over a stepper motor due to the need for a significant amount of starting torque. The inner face of the central rotator hub was fitted with nine 2-row sprocketed face plates spaced in $40^{\circ}$ increments around the rotator face. The AC motor was coupled to the sprocket rotator face using a steel double-stranded roller chain. The initial design of the rotator face only called for incremental face plates to be added to the rotator face, 
however in the rotator construction phase there were significant issues with sagging of the roller chain between segment. To address this issue spacer plates were added to the rotator face to provide support between sprocket segments. Because the roller chain length is given in increments of a single link, there was the possibility that it would not be sufficiently tight and slipping might occur when the chambers were being rotated. To address this problem the mount for the $\mathrm{AC}$ motor was made to be adjustable in 3 independent degree of freedom. During installation the motor was mounted and slack was removed from the chain; the motor was then shimmed and bolted into place. One thing that was extremely important with

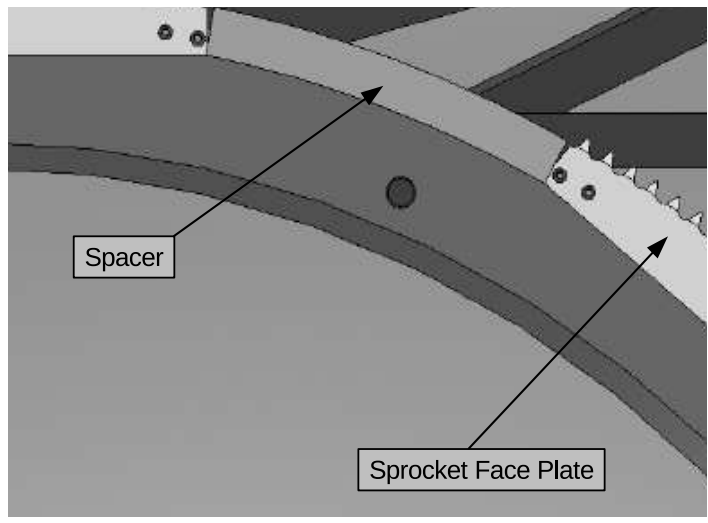

(a) Rotator face.

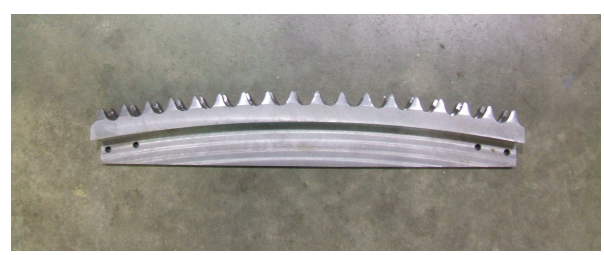

(b) Sprocketed face plate.

FIG. 3.24: Sprocketed plates placed along the rotator face were used along with a chain drive to turn the rotator.

the rotational motor was that rotation be done at low speed; included with the rotation motor was a speed reducer that provided a reduction of 249:1. This gave a maximum rotational frequency of $7.02 \mathrm{rpm}$. The original design criteria was that the rotational frequency be not more than $0.1-0.2 \mathrm{rpm}$. Further reduction was achieved by considering the gear ratio between the rotator hub and the size of the gear used for the rotational motor. Given the rotator "gear" radius of $2.895 \mathrm{~m}$, the motor gear was chosen to be $0.075 \mathrm{~m}$ giving a gear ratio of $0.026: 1$. This reduced the rotational 
frequency to a maximum of $0.183 \mathrm{rpm}$. The rotational motor was controlled using a variable frequency drive (HF-320 $\alpha$ Series). The drive could be controlled a number of ways, including: manual control using a potentiometer on the front panel, remote communication via RJ45 cable, or a 0 - $10 \mathrm{~V}$ terminal connection. The rotator controls system made use of both manual and automated control using terminal connections; details of the controls system set-up and implementation are discussed in more detail in Sect. 3.7.4. In the same manner as the linear arms, the rotator hub was locked in place after moving between octants. In each of the eight octants there was a 1 in. diameter pin hole on the upstream side of the rotator face (an example of this can be seen in Fig. 3.24a). In the same way as the linear arms, these pin holes were used to lock the rotator into the desired octant using an air-powered pancake cylinder. These cylinders drove a 1 in. diameter beveled steel pin into the pin hole. Confirmation of the pin being in was handled using a standard door jam switch - these are the same switches used in cars to detect whether a door is open. In each of the eight octants, on the backside of the upstream rotator face, a door jamb switch was mounted via a small hole drilled into the rear side of the rotary locking pin holes. When the rotational pin was inserted into the pin hole of a given octant, the door jamb switch was triggered and provided a $+5 \mathrm{~V}$ signal which could be read out by the controls system.

\subsubsection{Motion Controls System}

The motion controls system for the Region III was designed to provide a safe, effective way of controlling the linear and rotational motion of the rotator. Both the linear and rotational motor systems were manually operable, however the job of the control system was not only to provide a conduit to control this motion, but to 
provide synchronous linear motion, fail-safe controls, and more repeatability than is easily achieved when triggering motion by hand. The rotator controls system (Figure 3.25) was centralized in a standard controls rack located inside the entrance to the Region III shielding bunker. This location allowed operators to be in line-of-sight of the rotator while being safe from any possible failures. The main components of the controls system were the Versa Module Europa bus (VME) controls crate, Linear Motor Drives (S6961), and variable frequency drive. The VME crate contained an MVME6100 single board CPU, VME 612 Digital to Analog converter(DAC), a custom designed VME 16 channel relay board, custom 16 channel digital input register, and an MVME761 Serial input-output board. The linear and rotational locking pins

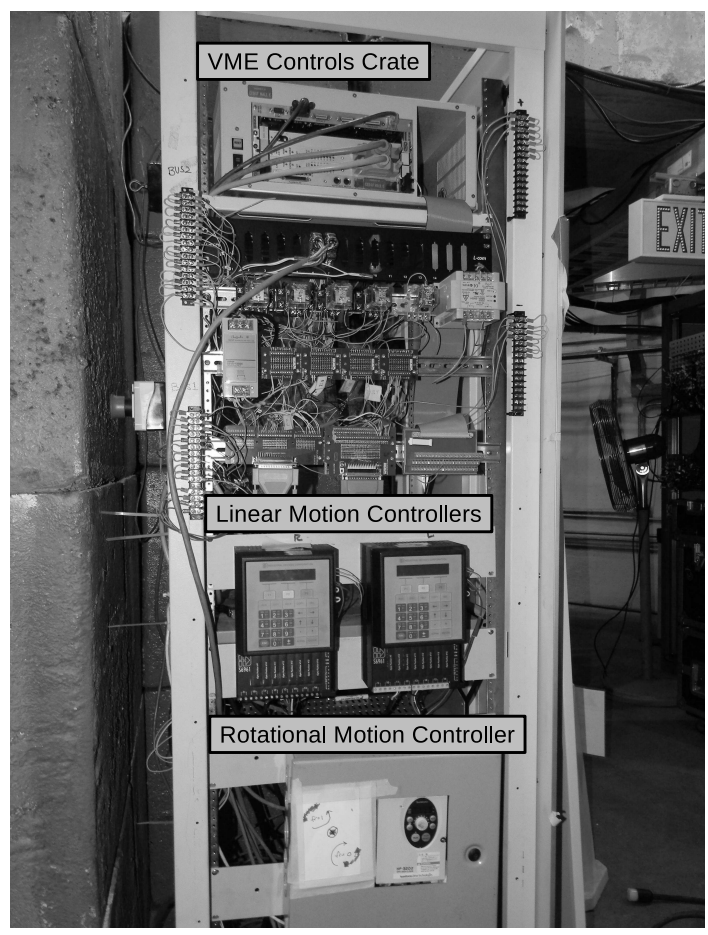

FIG. 3.25: Motion Controls Rack.

were operated using air driven pancake cylinders. Operationally, these cylinders are very simple; each cylinder has two valves, depending on the valve to which air was 
applied a central pin was moved in or out. The valve not having air applied to it became an exhaust valve. The air for these valves was routed from a high pressure source in the Hall $\mathrm{C}$ and was regulated to 100 psi - this is the recommended maximum pressure for these cylinders. The actuation of the pins was controlled by voltage switched pneumatic valves mounted on the inner hub of the rotator. Control of the linear motors was done using the rotator controls libraries running locally on the MVME6100 CPU. The controls libraries were loaded automatically when the controls crate was booted. Controls functionality was accessed by first using a secure shell (SSH) login to the MVME6100; functions in the rotator controls libraries were accessible from command prompt once an SSH session was initiated. Both software and hardware checks were applied to linear motion commands to ensure that the proper order of operations was observed during motion. Software monitoring was done through the 16 channel digital input register. The function of this board was to keep track of the voltage level on a given input channel, and therefore the state of the sensor attached to it. The state of each input was then accessible by reading the memory register associated with the channel. For the linear motion, the position of the VDC was cataloged and queryable via serial communication to the motor drives. Limits on the maximum and minimum position of the VDC were placed using Reed switches; the Reed switches operate on the principle of magnetic proximity. Once the motor drive shaft was moved to one of the limits, an internal magnet on the shaft triggered the normally-open (NO) Reed switch, stopping the motor. In order for linear motion to be possible three conditions were required: the rotator had to be in the horizontal position, the rotational locking pin must be inserted, and the linear locking pins had to be removed. The horizontal position was checked using a tilt sensor which was normally-open in any position other than horizontal. The checking of the rotational pin was done using the pin-in sensor located inside of each 
rotational pin hole. The linear locking fail-safe was managed using both the input register and the relay board. When the software command was given to engage the linear locking pins the associated relays were closed on the board which applied power to the pneumatic valves managing the air-driven pancake cylinders. Due to the possibility of software failures, the linear motion system controls were tied to a physical relay AND of the aforementioned conditions. The power to the linear motor was wired to a relay in the NO position. Power to this relay was physically tied to all three of the conditions above being met; in this way the linear motors were shut off completely until it was safe to move them. Similar to the implementation of the linear motion controls the rotation controls were given both software and hardware fail-safes. The rotational motions controls code used read-back from the rotational locking pin, linear locking pins, and linear motor brake to determine whether or not it was safe to rotate. As an added level of precaution the rotational motor controls were also tied to a physical relay fail-safe. The conditions for the rotational motion to be operational were: power applied to the linear locking pins, rotational locking pin in the out position, and the brake applied to the linear motor. The power to the variable frequency drive that controlled the rotational motor, as well as powering it, were tied to a high voltage relay; power to this relay was dependent on a physical AND of the relays controlling the preceding conditions. Originally the rotational controls system were designed to be much more automated. When first implemented, the controls system kept track of what octant the rotator was in, and could be moved to other octants simply by giving the command and desired octant number. This mode of operation was not used during the experiment, however, for two reasons: 1) during installation the support bracket holding the tracking sensor was mis-welded. and 2) the rotation of such a large structure, in a region containing not only the VDC packages but the main detector for the experiment was deemed 
much safer if done by hand. No longer using the sensor that provided a read-back of the rotational position made moving to a given octant and securing the rotational locking pin more difficult. With this in mind, an improvised positioning system was constructed. A number of standard laser pointers were mounted on the main detector support structure (see Fig. 3.26). While the rotator arms were in the extended position, survey points were marked on the VDC plates and rotator arms where the laser spots were; by trying to align to multiple points in each octant we hoped to decrease the error on the repeatability in each octant. The size of each laser spot was $\sim 1 \mathrm{~mm}$ diameter which gave an upper limit on how well we could ensure the rotational repeatability.

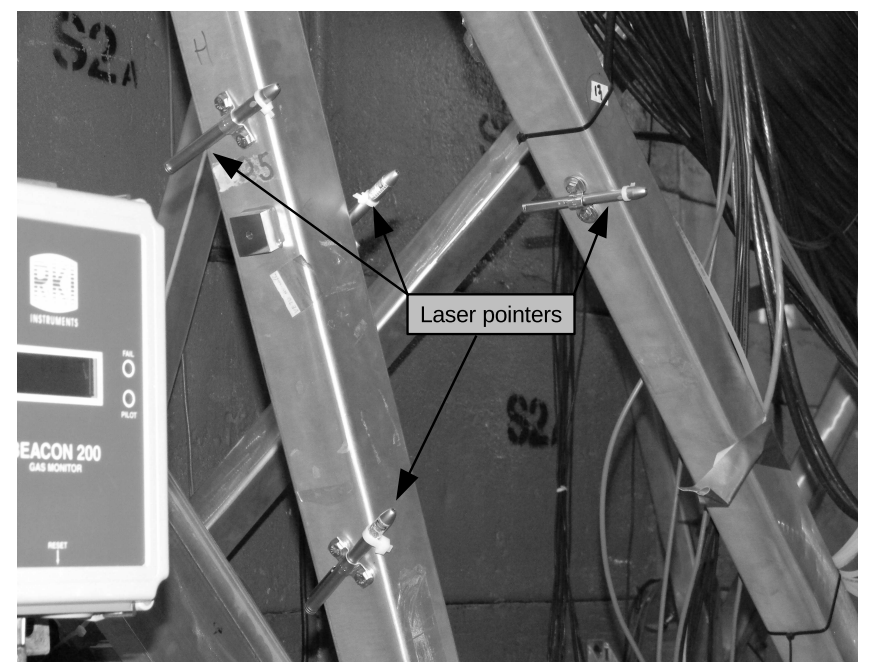

FIG. 3.26: Laser pointers mounted on main detector support structure.

\subsubsection{Performance and Repeatability}

During the 2 year run period of the experiment, the region III rotator performed well, being one of the few subsystems with no major failures. Along with reliability, the key metric for the performance of the rotator was its repeatability; poor 
repeatability increases the error on how well we are able to determine the scattered electron trajectory through the chambers. Both during commissioning and between run periods repeatability studies where completed with the help of the survey group at JLab. A listing of rotator surveys as well as notes about the program of each can be found here[61]. The survey study presented in this thesis was completed in September 2010 prior to the completion of the commissioning running. The results of the study showed the radial consistency between octants, i.e. the difference in the measured radius of each measurement point as the VDCs were rotated between octants, to be sub-millimeter in the horizontal position and at the millimeter level in the vertical position. The radial numbers were larger when only considering the xy-plane (perpendicular to the beam direction) indicating the VDCs were tilted in the direction of the beam. The angular repeatability when rotating the VDCs away from the horizontal position and back was found to be on the millidegree level. The repeatability of the VDC after being retracted and extended was found to be good to the $100 \mu \mathrm{m}$ level. Lastly, the distance between measurement points was studied. The survey is done by attaching tooling balls in special positions on the face of the VDC packages, because these tooling balls are mounted in static locations the distance between them should not change within the resolution of the survey measurement and any contributions from potential sagging or flexing of the chambers. The positional difference between points was found to be good to the micron level. The full summary of results as well as a more in-depth discussion of each measurement can be found in A.2. The results of the rotator study show that the repeatability of the chambers position was well within the limits of what is required of our $\mathrm{Q}^{2}$ measurement. 


\section{CHAPTER 4}

\section{Beam Modulation}

\subsection{Modulation System}

\subsubsection{Beam Modulation and Helicity-Correlated Beam Sys- tematics}

One of the more challenging aspects of PVES experiments is suppression and measurement of helicity-correlated beam systematics (HCBS). These changes in the beam intensity, position, profile, and energy - when correlated with the helicity of the beam - create false asymmetries that distort the measurement of the physics asymmetry. These false asymmetries are especially important in precision experiments due to the small asymmetries that are being measured. One of the primary source of HCBS is imperfections in the laser polarization; laser light on the photo-cathode - while highly polarized - can contain small components of linear polarization which switch sign under helicity flip. This, along with anisotropy in the quantum efficiency of the photo-cathode, can cause helicity-correlated differences in the intensity of po- 
larized electrons being produced from the photo-cathode. This shows up as a charge asymmetry in our measurement. Other sources of HCBS include lensing, where the Pockel cell operates as an electro-optical device deflecting the beam on helicity flip, electronic noise, and helicity signal leakage in the injector[4]. A number of well established methods were used to remove or heavily suppress HCBS; methods such as the RHWP and the IHWP work to reduce HCBS sources both before and after the Pockels cell as well as providing a diagnostic which helps determine their origin.

The central purpose of the following section is the determination of the detector sensitivity to helicity-correlated beam motion using the beam modulation system. The main benefit of using the beam modulation system was that it provided a measurement that was directly correlated to the driven motion of the beam. The alternative method, linear regression of natural beam motion, provides an effective way to reduce the asymmetry width and remove correlation of the detector response to beam jitter, however the meaning of these corrections is not well-posed. Take for example a hidden variable, such as halo, that is correlated to the jitter in the beam on helicity flip; this would give the extracted sensitivities a different character detracting from our understanding of the HCBS. The result with modulation is much clearer: removal of the correlation of detector signal to a controlled beam motion. Hidden variables should not contribute, and any residual correlations would indicate non-linearities, measurement failures, or hidden variables.

\subsubsection{Beam Modulation System Instrumentation}

As mentioned in Sect. 3.5.6, the beam modulation system was (schematic shown in Fig. 4.1) used to purposely modulate the electron beam in a controlled way. The goal was to provide a system that could produce roughly independent offsets in 
each of the beam parameters, allowing for accurate determination of the detector sensitivities on the scale of hours, as well as suppressing contributions from intrinsic correlations in the beam. The system consisted of four pairs of air-core inductive copper coils - two pair for X-like motion and two for Y-like motion - placed along the beamline (Fig. 4.2). Each coil was driven by the output of a 16 bit VMIVME-4145 Waveform Generator. These waveform generators provided 4 channels of output per board and have options for both internal or external triggering. For each channel the sinusoidal waveform was built using a $64 \mathrm{k}$ word sample buffer. Once a waveform of the desired frequency was lo added into the sample buffer, the external signal was used to trigger the output on each desired channel. The signal output of each channel was split, with one copy sent through a JLab-designed power amplifier card $\left(\right.$ TRIM-I $\left.^{1}\right)$ and used as a driving signal for the modulation coils. The other copy was sent to an ADC, where it was used as both a diagnostic and as a reference signal to lock the measured beam monitor response to the driving signal. The later point became a powerful tool in the analysis methodology when dealing with the effects of the fast-feedback system (FFB) on the beam monitor response to modulation. Energy modulation was carried out in a similar fashion to position modulation, with a driving signal being sent to the input of a superconducting radio-frequency (SRF) cavity in the south linac of the accelerator. By modulating the input voltage to the cavity we changed the electric field in the cavity and thereby the energy of the electron beam. A final output channel was dedicated to producing a linearlyincreasing periodic function we defined as a "ramp" signal that was run directly into an ADC. Since all outputs of the waveform generator were triggered via a single external signal, the output wave-forms were synchronous. For every trigger

\footnotetext{
${ }^{1}$ Originally we had planned on using the second generation version of this power amplifier, however the engineering group at JLab found problems with them and they were removed.
} 
that produced a driving signal the ramp channel was also triggered; through this we were able to determine the phase of the driving signals.

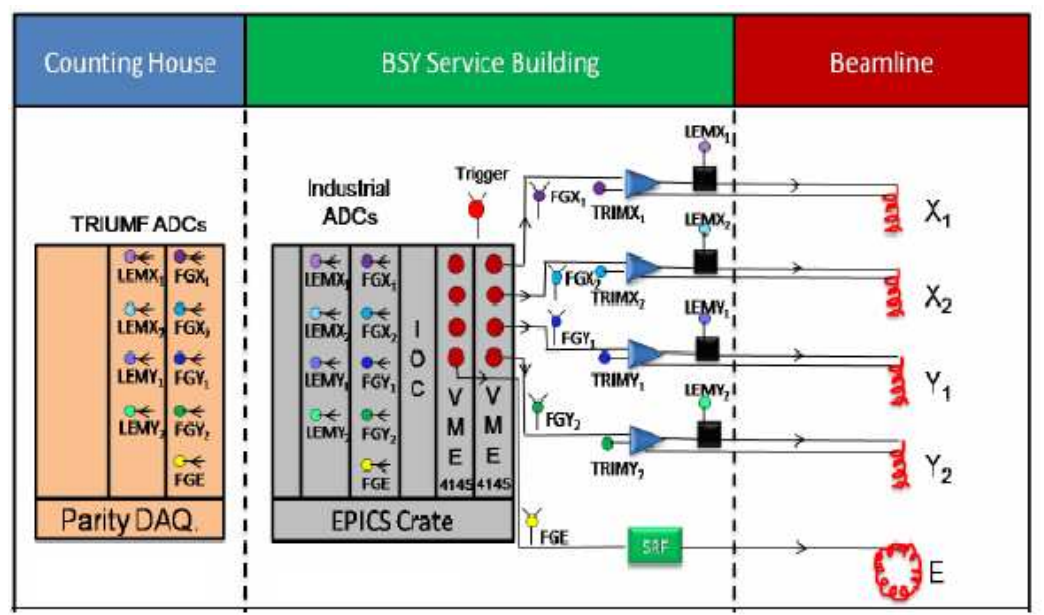

FIG. 4.1: The modulation coil pairs are shown in red on the far right. Each coil was driven by an amplified signal from the VMIVME-4145 wave-form generators seen in the center. A second copy of the driving signal was recorded by the main DAQ[11].

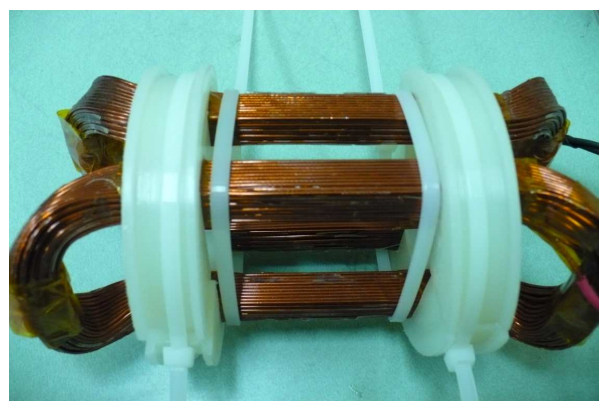

(a) Air-core coil used for driven modulation.

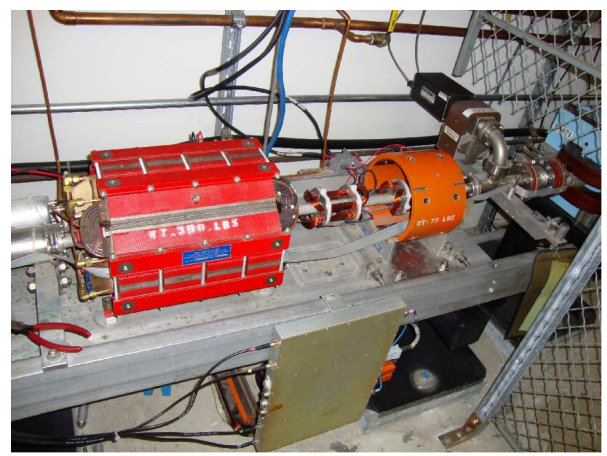

(b) Air-core coils in position on the beamline.

FIG. 4.2: Copper air-core coils used in the modulation system.

During the design of the beam modulation instrumentation it was important to determine whether the modulation hardware was capable of providing the required field integrals, at the frequencies desired. Both the frequency and type of waveform modulation were important to these tests. We wanted to maximize the up-time of our measurement at maximum amplitude. Higher frequency modulation gives 
a higher number of measurements per minute and using a waveform that spends more time at maximum displacement makes each measurement more precise, e.g. a square wave would be preferable over a sine wave. Bench tests were performed using the air-core coils driven using a FANUC VME function generator with the TRIM-II power amplifiers. During the bench test the coils were modulated at frequencies of $10 \mathrm{~Hz}-500 \mathrm{~Hz}$; a Hall probe was used to measure the field integral and the power amplifier input voltage versus the coil current was recorded for different frequencies. As a result of the bench top tests it was determined that the maximum reliable operating conditions for the system was $250 \mathrm{~Hz}$ at a current of $3 \mathrm{~A}$ [62]. The choice was made during Run I to operate the modulation system at a frequency of $125 \mathrm{~Hz}$ - this is slightly off the poles of the FFB system which cancels beam noise at $60 \mathrm{~Hz}$ and higher harmonics. This choice of sampling frequency provided an acceptable waveform while providing sufficient livetime for each modulation cycle.

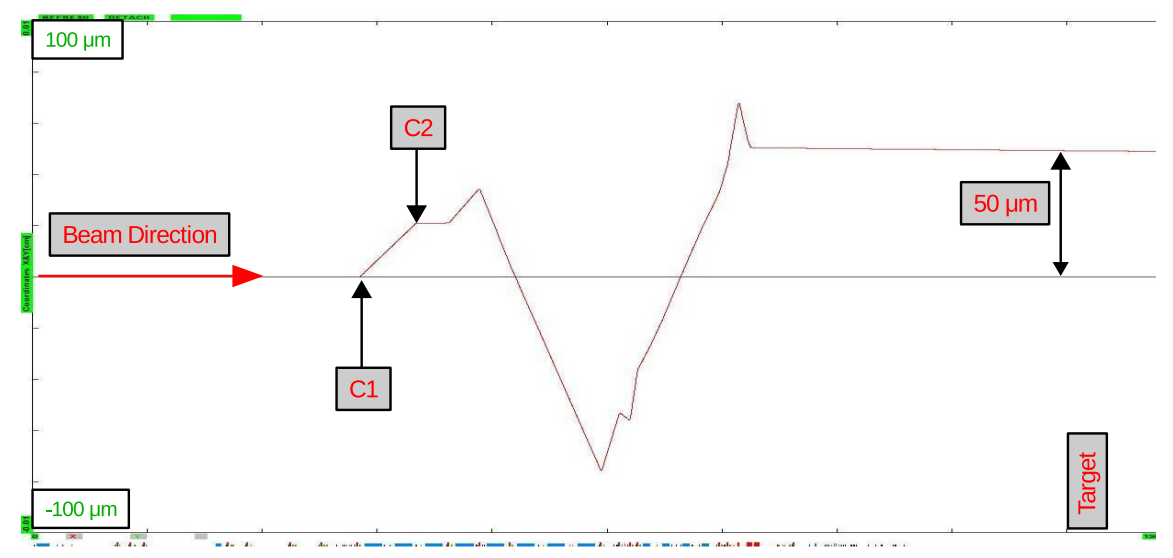

FIG. 4.3: An example beamline optics simulation to generate a $50 \mu \mathrm{m}$ offset at the target. The red arrow shows the direction of the beam. The coils are represented by $\mathrm{C} 1$ and $\mathrm{C} 2$ and show the optimal locations along the beamline to apply kicks[12].

The position, angle, and energy of the beam at the target is determined by the transfer function of the beam from the modulation coils to the target. By applying 
the proper set of kicks to the beam at strategic locations along the beamline we were able to generate the desired offsets at the target. The ratio and amplitude of the kicks for each modulation type were determined using beam transport simulations in OpTIM[63]. Taking advantage of the time reversal invariance of the electromagnetic interaction, the beam was run backwards through the simulation, starting in the desired position and moving upstream along the beamline. An example optics simulation for an initial $50 \mu \mathrm{m}$ offset at the target can be seen in Fig 4.3. Here the beam moves from left to right, as indicated by the red arrow, propagating upstream along the beamline. The simulated path shows the orbit, given the transport optics, the beam would need to result in a pure position offset of $50 \mu \mathrm{m}$ at the target. The zero-crossings of the time reversed beam are important in the sense that they provide a direct mapping of the position(angle) at the target to the beam angle at the coil position. Placing kicker coils at these locations allowed us to generate the forward orbit resulting in the pure position(angle) offset we desired. Similar simulations were carried out for each type of modulation; these simulations determined the starting currents and coil ratios that would be used during running. One of the default running configuration can be found in Table 4.1. In the table you can see the coil currents required to produce the given offsets; one important thing to notice is the near degeneracy in the Y-like coil currents. The basic concern here was that if two or more beam parameters - which make up our basis set - were not sufficiently independent there could be difficulty in extracting the sensitivities. This was an ever present concern throughout the extent of the experimental running, and one that was never completely resolved. During times that a change in accelerator optics was suspected, plots of the beam trajectory were made by plotting the BPM response to modulation to each kind of modulation as a function of $\mathrm{Z}$ position along the beamline. These optics plots provided a snapshot of the current beam optics, 


\begin{tabular}{|l|c|r|r|l|}
\hline Parameter & Amplitude & $\mathrm{C}_{1}$ Current $(\mathrm{A})$ & $\mathrm{C}_{2}$ Current $(\mathrm{A})$ & Coil Ratio $\left(\mathrm{C}_{2} / \mathrm{C}_{1}\right)$ \\
\hline $\mathrm{X}$ & $159.0 \mu \mathrm{m}$ & 0.088 & -0.300 & -3.414 \\
\hline $\mathrm{X}^{\prime}$ & $3.1 \mu \mathrm{rad}$ & 0.052 & -0.300 & -5.824 \\
\hline $\mathrm{Y}$ & $84.0 \mu \mathrm{m}$ & -0.300 & 0.136 & -2.200 \\
\hline $\mathrm{Y}^{\prime}$ & $3.1 \mu \mathrm{rad}$ & -0.300 & -0.150 & -2.000 \\
\hline
\end{tabular}

TABLE 4.1: Example of a standard run configuration from Run I modulation. These numbers were adjusted throughout the experiment as the beam optics changed.

and provided a convenient way to investigate any position-angle mixing that might have been present. In order to address these optics changes and try to optimize the response, special tune-scan runs were taken in which the coil tune would be varied by $\pm 10 \%$. The resulting change in the optics was then studied and a "best" tune value was extracted.

The controls system for the beam modulation system was written in State Notation Language (SNL) using sequencers. SNL is a powerful programming framework that is useful in real-time control systems; the run-time sequencer drives the controls system into different states based on different events and relieves the complexity of task scheduling and event handling that is a staple of a real-time multi-tasking environment. Both SNL and the sequencer are components of a larger controls framework, the Experimental Physics and Industrial Controls System (EPICS $\left.{ }^{2}\right)$. EPICS is an interactive development toolkit and real-time controls environment for physics and industrial applications. The sequencer setup was especially important to the modulation system as it allowed monitoring of the modulation related variables in a simple way as well as scheduling of different modulation cycles. Once initialized, the controls system would read in the desired modulation setup from a user defined configuration file - this configuration file would include which channels were to be initialized, the frequency at which to modulate, and the driving signal

\footnotetext{
${ }^{2}$ http://www.aps.anl.gov/epics/
} 
amplitude defined in amps. Once the configuration files had been read-in, the waveform generator boards were transitioned into a ready state. An external trigger was then applied to the boards; this signal would cause the channels that were initialized for a certain modulation type to trigger and run through a full set of cycles. Once the modulation cycles were finished, the system would set all channels into an OFF state and move to a wait state until it was time to wake up and modulate again. The modulation system ran continuously during most of the production running, stepping through a modulation sequence in each beam parameter; a set of microcycles containing one instance of modulation in each beam parameter makes up a macro-cycle. During each modulation cycle a pattern number flag was set in the DAQ; there was a unique pattern number for modulation in each beam parameter, which allowed us to identify the type of modulation of each micro-cycle during analysis. The modulation sequence consisted of driving each pair of modulation coils in micro-cycles of about 4 seconds, or 512 cycles at $125 \mathrm{~Hz}$. There was a down time of $\approx 75$ seconds between each micro-cycle of modulation which was needed to reconfigure the boards for the next modulation type. A full modulation macro-cycle was 320 seconds, after which time the modulation system would reconfigure and repeat the macro cycle; the modulation data composed about $5 \%$ of our production running.

\subsubsection{Methodology}

As mentioned previously, measurement and correction of false asymmetry caused by helicity-correlated beam systematics was done using two methods: using standard linear regression technique applied to natural beam jitter, and using large-amplitude driven beam motion via the beam modulation system. The correction to the raw 


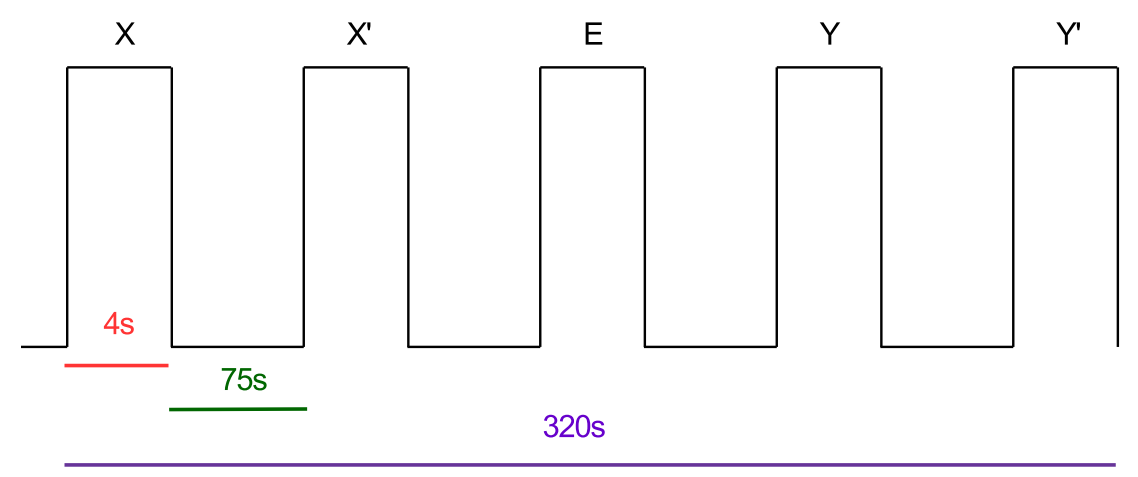

FIG. 4.4: Schematic of beam modulation cycle timing. Each pulse section is 512 cycles of sinusoidal function at frequency of $125 \mathrm{~Hz}$. Pulse are broken down into micro-cycles that make up a full macro-cycle.

asymmetry due to helicity-correlated beam systematics was determined using the beam modulation system outlined in the previous section. The raw asymmetry was corrected for false asymmetries according to

$$
A_{\text {corrected }}=A_{\text {raw }}-A_{\text {false }}
$$

where $A_{\text {false }}$ is the correction due to false asymmetries and is given by

$$
A_{\text {false }}=\frac{1}{\langle Y\rangle} \sum_{i=1 \ldots 5} \frac{\partial Y}{\partial X_{i}} \Delta X
$$

Here the detector sensitivities are given by $\partial Y / \partial X_{i}$ and represent the direct correlation of the detector yield to changes in a given beam parameter. It is important to note that this is not directly measurable; the beam modulation strove to provide linearly independent modulations of the beam but could not completely remove cross correlations between beam parameters, e.g. position modulations also had components of energy and angle mixed in. Extraction of the detector sensitivities was done by 
considering the response of the detector yield with respect to the modulation signal; the modulation signal is analogous the modulation type. Expanding with respect to the beam parameters,

$$
\frac{\partial Y}{\partial C_{i}}=\sum_{j=1 \ldots N} \frac{\partial Y}{\partial X_{j}} \frac{\partial X_{j}}{\partial C_{i}}
$$

where $C_{j}$ represents a time-dependent signal synced with the modulation driving signal, yields the detector sensitivities. The beam monitor matrix, given by $\partial X_{j} / \partial C_{i}$, gives the correlation of the beam parameters measured in the monitors to each modulation type. Both the beam parameter correlation matrix and the detector response to each modulation type are directly measurable. The detector sensitivities can then be extracted by matrix inversion:

$$
\frac{\partial Y}{\partial X_{j}}=\frac{\partial Y}{\partial C_{i}}\left[\frac{\partial X_{j}}{\partial C_{i}}\right]^{-1}
$$

This can be written in matrix form as:

$$
\mathbf{M}_{\mathrm{S}}=\mathbf{M}_{\mathrm{C}} \mathbf{M}_{\mathrm{M}}^{-1}
$$

As was explained in Sec. 4.1.2, the beam modulation system used pairs of coils, driven with different amplitudes, which produced a composite response in the beam. While the individual driving signals were available in the DAQ, there was no readback of the composite driving signal. Instead the ramp signal, which was synced to the coil driving signals and common to each modulation type, was used. Refering to Eq. 4.3 above, $C_{j}$ is a parametrized version of the ramp signal given by

$$
C(t)_{i}=\sin (\alpha r(t)-\phi)
$$


where $r(t)$ is the ramp function. Parametrizing the response in this way allowed us to pick out only the component of the response directly proportional to the driving signal, which was important in circumventing components in the response such as Fast-Feedback (FFB) system, discussed later in this chapter. Using the detector response in Eq. 4.4, the sensitivities to each modulation type $\left(\mathbf{M}_{\mathrm{C}}\right)$ were determined using a fit to a linear regression line,

$$
M_{\mathrm{C}}^{j}=\frac{\sum_{i=1 \ldots N}\left(C_{i}^{j}-\left\langle C^{j}\right\rangle\right)\left(Y_{i}^{j}-\left\langle Y^{j}\right\rangle\right)}{\sum_{i=1 \ldots N}\left(C_{i}^{j}-\left\langle C^{j}\right\rangle\right)^{2}},
$$

where the sum is over the $\mathrm{N}$ modulation events in a micro-cycle, and $j$ is the modulation type. The correlation matrix $\left(\mathbf{M}_{\mathrm{M}}\right)$ was calculated in a similar fashion for each modulation type and beam monitor as,

$$
M_{\mathrm{M}}^{j k}=\frac{\sum_{i=1 \ldots N}\left(C_{i}^{j k}-\left\langle C^{j k}\right\rangle\right)\left(M_{i}^{j k}-\left\langle M^{j k}\right\rangle\right)}{\sum_{i=1 \ldots N}\left(C_{i}^{j k}-\left\langle C^{j k}\right\rangle\right)^{2}} .
$$

Here $j$ represents the modulation type, and $k$ is the beam parameter. This was done for each micro-cycle in a given run and the error weighted average of $\mathrm{M}_{\mathrm{C}}$ and $\mathrm{M}_{\mathrm{M}}$ were used in Eq. 4.5 to complete $\mathbf{M}_{\mathrm{C}}$ and $\mathbf{M}_{\mathrm{M}}$ respectively. Given that $\mathbf{M}_{\mathrm{M}}$ is invertible, i.e. $\left|\mathbf{M}_{\mathrm{M}}\right| \neq 0$, the detector sensitivities to each beam parameter $\left(\mathbf{M}_{\mathrm{S}}\right)$ can be extracted as shown in Eq. 4.5 - 4.6.

One of the complications that came about when syncing to the ramp signal was the way in which the ramp signal was sampled. The DAQ samples signals coming into the ADCs at $960 \mathrm{~Hz}$, and given the modulation frequency of $125 \mathrm{~Hz}$, there are $\sim 8$ data points for each ramp cycle. The issue comes about at the transition point between ramp cycles; when the ramp signal transition falls in the center of an ADC sample window, it creates a point that is not consistent with the functional form 
given by $r(t)$. In Fig. 4.5 a sample output of the ramp signal is shown; depending on how close to the center of the ADC window the transition point falls the ramp signal coincides with a different phase. The results of this can be seen by plotting one of the modulation driving signals versus ramp, as can be seen in Fig. 4.6. The seemingly linear function that tracks through the center of the driving signal is due to the ramp transition points falling in progressing different places in the ADC window. In order to remove this effect, a simple linearity test was applied in the modulation analyzer. This test takes advantage of the fact that for each ramp point in the ADC the four subblocks used to build that point are also saved. Using the subblocks, a linearity test of the form,

$$
\mid(\text { ramp_block0 }+ \text { ramp_block2 })-(\text { ramp_block1 }+ \text { ramp_block3 }) \mid<\delta \text { r }
$$

was used. The size of $\delta r$ was determined analytically to be $50^{\circ}$, which removed the majority of the transition points while not being so strict a cut as to remove large amounts of data. Referring again to Fig. 4.6, the results of the above cut can be seen as the points in black. The points removed by this cut become an important systematic in the determination of the detector sensitivities; by removing these points, a phase gap is created in the modulation data. The details of how this effects the determination of the detector sensitivities can be found in Sec. 4.1.4. In later analyses a better way to deal with this effect was developed which actually reassigned the points calculated at the transition point to where they are expected in the phase gap according to the linear nature of the ramp function[64]. This method was not applied in this analysis and won’t be discussed further.

Early in the experiment, during commissioning of the modulation system, it was decided that unlike previous implementations of similar systems $[65,66]$, FFB 


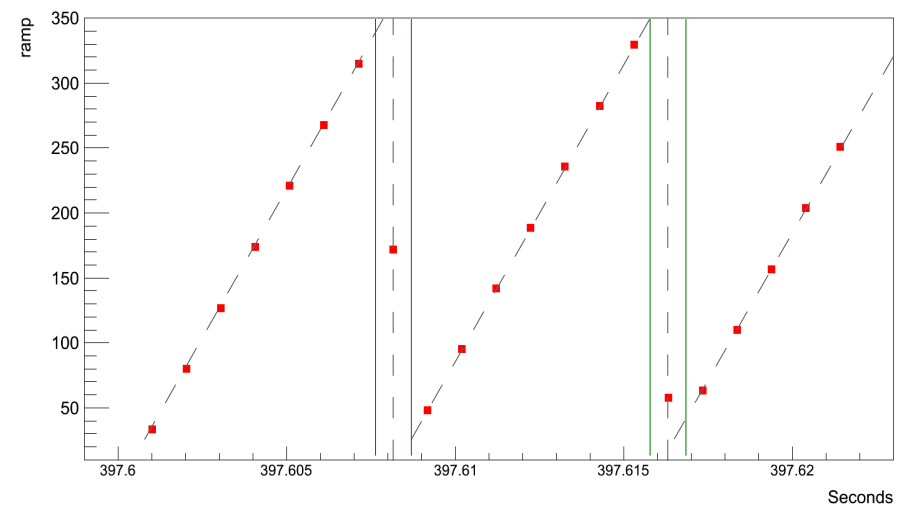

FIG. 4.5: The ramp signal read out by the ADCs. Because the DAQ samples at a rate of $980 \mathrm{~Hz}$ and the ramp signal has a frequency of $125 \mathrm{~Hz}$, the ADC records 8 points per cycle. When the ramp transition falls in the middle of an ADC sample window the ramp point recorded does not match up with what is expected at that time; see data surrounded by green vertical lines.

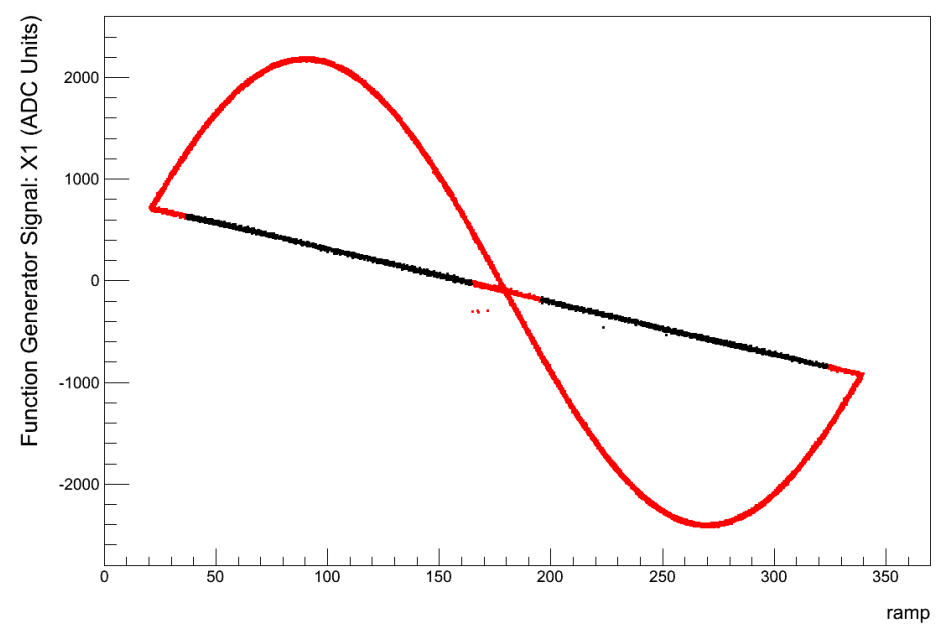

FIG. 4.6: An example plot of the modulation driving signal for one coil plotted versus the ramp function is shown in red. The edge effects of the ramp signal being sampled by the ADC point recorded at an incorrect phase form a linear function that tracks through the center of the driving signal. Using a simple linearity cut many of these points (shown in black) can be removed. 


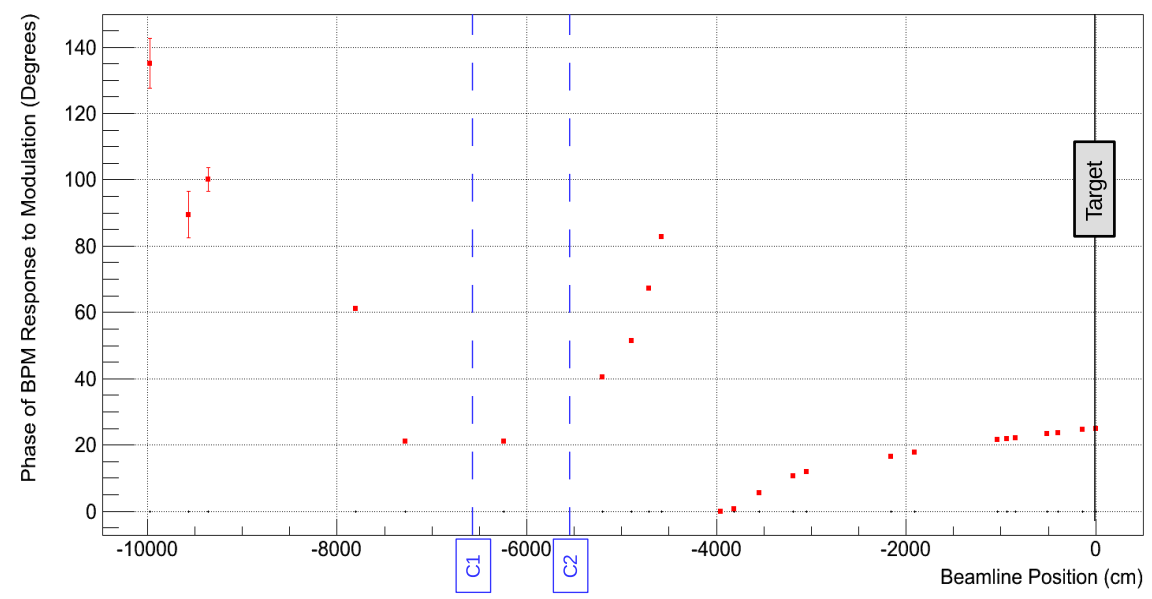

FIG. 4.7: The phase of the BPM response is shown as a function of position along the beamline. The dashed blue lines indicate the position of the vertical and horizontal modulation coils.

would be enabled during running, except during energy modulation. This was due to fears that disabling the FFB system, which actively monitored and tried to correct beam noise, would introduce significant noise into the experimental measurement. This decision came following a test run of the modulation system which showed two things [67]: the energy FFB completely flattened the energy modulation signal and the suppression of the position modulation was small $(\mathcal{O}(5 \%))$. Unfortunately, the effect on the phase of the BPM response to modulation was not recognized during the commissioning tests.

In Fig. 4.7 the phase of the BPM response in each beam monitor along the beamline is shown; of primary importance are the final five BPMs before the target which are used to construct the "target" variables and therefore directly affect the extraction of the detector sensitivities. To understand the phase shift due to FFB we looked at how the FFB algorithm monitored and made corrections to the beam position. Along the beamline certain BPMs were monitored by the FFB system. 
These feedback BPMs were used by the FFB system, which samples the position noise over two second windows at $1.8 \mathrm{kHz}$, and creates a waveform to null out horizontal (vertical) displacement with an equal but opposite position offset. This is equivalent to another modulation coil that has a delayed response - or a different phase - to the modulation. The total response of the BPM to modulation can be parametrized as the sum of two harmonic functions [68] with different phases

$$
X(t)=A \sin \left(\omega t+\phi_{\mathrm{mod}}\right)+B \sin \left(\omega t+\phi_{\mathrm{ffb}}\right)
$$

This can be be rewritten as

$$
X(t)=A^{\prime} \sin (\omega t+\alpha)
$$

where

$$
\begin{gathered}
\alpha=\tan ^{-1} \frac{A \sin \phi_{\mathrm{mod}}+B \sin \phi_{\mathrm{ffb}}}{A \cos \phi_{\mathrm{mod}}+B \cos \phi_{\mathrm{ffb}}} \\
A^{\prime}=\sqrt{\left(A \sin \phi_{\mathrm{mod}}+B \sin \phi_{\mathrm{ffb}}\right)^{2}+\left(A \cos \phi_{\mathrm{mod}}+B \cos \phi_{\mathrm{ffb}}\right)^{2}} .
\end{gathered}
$$

Here $\phi_{\mathrm{ffb}}$ is the response of the FFB to the modulation signal and $\phi_{\text {mod }}$ is the composite response of the beam to modulation. The problem this presents is that the phase of the composite response is a function of the relative phases and amplitudes of the modulation coils and the FFB system. The amplitude response of a given BPM to modulation depends on the transfer function from the coils to a particular point along the beamline. This means we had a position-along-the-beamline dependent phase response to modulation; another way of saying this is that we are spanning more than one dimension in phase space. The solution to this was that we measured the response to modulation with a fixed phase. Considering the response to a given 
modulation as the sum of harmonic functions, we have

$$
X(t)=\sum_{i=0 \ldots N} x_{i} \sin (\omega t+\phi)=x_{\mathrm{s}}^{\mathrm{ffb}+\text { drive }} \sin \left(\omega t+\phi_{\mathrm{o}}\right)+x_{\mathrm{c}}^{\mathrm{ffb}} \sin \left(\omega t+\phi_{\mathrm{o}}\right)
$$

where $x^{\mathrm{ffb}+\text { drive }}$ is the component of the response due to the driving signal plus the

sine component of the FFB, and $x_{\mathrm{c}}^{\mathrm{ffb}}$ is the cosine component of the FFB response. If we consider only the sine component of the BPM response, the FFB-only component averages out, leaving us with an amplitude that is proportional to the driving signal. In fact, because the two harmonic functions are time-orthogonal, the FFB component will always cancel out, allowing us to use any arbitrary phase. For the purpose of the analysis presented here, $\phi$ was chosen to be zero. This corresponds to $\phi=0$ in Eq. 4.6. This postulate rests completely on the assumption that the FFB response is not dynamic on the time-scale of the modulation. A detailed presentation of how this phase lock postulate was tested is presented in Sec. 4.1.4.

\subsubsection{Modulation Regression Analysis}

The previous section explained the basic methodology used, as well as some of the difficulties encountered, in the measurement of the detector sensitivities using the beam modulation system. This section details the diagnostics used to ascertain the quality of the extracted sensitivities and the subsequent corrections to the measured asymmetry. In Sec. 4.1.3 it was explained that due to the FFB being left on during modulation, there was a phase shift induced into the BPM response used to calculate the detector sensitivities. The proposed solution was to model the total BPM response as the superposition of a set of harmonic functions, which could be separated into components proportional to the driving signal plus part of the FFB, and a time-orthogonal piece proportional only to the FFB signal. This led 
to the postulate that we could choose any arbitrary phase to extract the detector sensitivities. To test this, four different "sets" were chosen - each with a different set of phases - to extract the detector sensitivities. Shown in Table 4.2 are the different phases used in the modulation analysis.

\begin{tabular}{|l|c|c|c|c|c|}
\hline Phase $(\mathrm{deg}) /$ Set & $\phi_{X}^{n}$ & $\phi_{X^{\prime}}^{n}$ & $\phi_{3 c 12 X}^{n}$ & $\phi_{Y}^{n}$ & $\phi_{Y^{\prime}}^{n}$ \\
\hline Set 1 & 0.0 & 0.0 & 0.0 & 0.0 & 0.0 \\
\hline Set 2 & 0.0 & 0.0 & 0.0 & 0.0 & 90.0 \\
\hline Set 3 & 0.16 & 0.12 & -1.15 & 0.12 & 0.14 \\
\hline Set 4 & 14.89 & 14.89 & 0.0 & 61.88 & 61.88 \\
\hline
\end{tabular}

TABLE 4.2: List of phases used in the different modulation sets, in units of degrees.

Sets 1 and 2 were chosen for simplicity and with the intent of trying to separate the $\mathrm{Y}$ and $\mathrm{Y}^{\prime}$ beam parameters. Set 3 and 4 were chosen by considering parts of the correlation matrix which significantly contribute to the extracted sensitivities; the phase choices for these sets were chosen to remove or suppress phase offsets in these high contribution areas. Table 4.3 shows the results of the Wien-Average sensitivities for each set during Run I. Qualitatively, the extracted sensitivities of a given type are the same, however they do not match within the error bars. The variation from set to set is most likely due to strength sharing between the extracted sensitivities. Normally it would not be expected for there to be a correlation if the basis has been properly defined, however this is only true if the definition of the basis vectors being used do not change. Throughout the experimental running there

\begin{tabular}{|l|l|l|l|l|l|}
\hline DOF/Set & $\mathrm{X}$ & $\mathrm{X}^{\prime}$ & $3 \mathrm{c} 12 \mathrm{X}$ & $\mathrm{Y}$ & $\mathrm{Y}^{\prime}$ \\
\hline Set 1 & $-1685.3 \pm 6.7$ & $81.9 \pm 0.3$ & $-1478.1 \pm 1.2$ & $259.7 \pm 4.8$ & $-1.7 \pm 0.2$ \\
\hline Set 2 & $-1685.5 \pm 6.2$ & $82.5 \pm 0.3$ & $-1491.9 \pm 1.5$ & $306.3 \pm 6.9$ & $-5.5 \pm 0.5$ \\
\hline Set 3 & $-1686.8 \pm 7.3$ & $81.8 \pm 0.3$ & $-1490.7 \pm 1.8$ & $249.2 \pm 5.9$ & $-2.5 \pm 0.3$ \\
\hline Set 4 & $-1538.4 \pm 9.4$ & $80.0 \pm 0.7$ & $-1473.4 \pm 3.7$ & $267.0 \pm 26.6$ & $-0.1 \pm 1.6$ \\
\hline
\end{tabular}

TABLE 4.3: Wien average sensitivities for each modulation set. Positions sensitivities are in units of $\mathrm{pm} / \mathrm{mm}$ and angle sensitivities are in units of $\mathrm{ppm} / \mu \mathrm{rad}$. 
was significant dispersion in the beam-tune, increasing the correlation between the position and energy of the beam; this correlation changed as the beam tune drifted. In this model, the definition of $\mathrm{X}, \mathrm{X}^{\prime}$, and $\mathrm{E}$ changes and therefore a slight shift in the extracted sensitivities is expected. In Fig. 4.8 - 4.12 you can see correlation plots of the virtual target BPMs and bpm3c12X which demonstrate the strength sharing present in the extracted sensitivities. Each plot shows the set of Run I modulation extracted sensitivities on the runlet basis $^{3}$ for each monitor.

One likely cause of the correlation of the extracted sensitivities was correlated noise in the monitors used to form the virtual BPMs as well as bpm3c12X; with this in mind we expect the correlation to be suppressed when averaging over longer time scales. Correlation plots of the Slug-averaged sensitivities are shown in Fig. 4.13 4.17, and show that the correlation between the extracted sensitivities is suppressed as the monitor noise is averaged out, as expected.

\footnotetext{
${ }^{3}$ In this case runlet refers to segments of a full run, each segment making up approximately 6 minutes of data.
} 


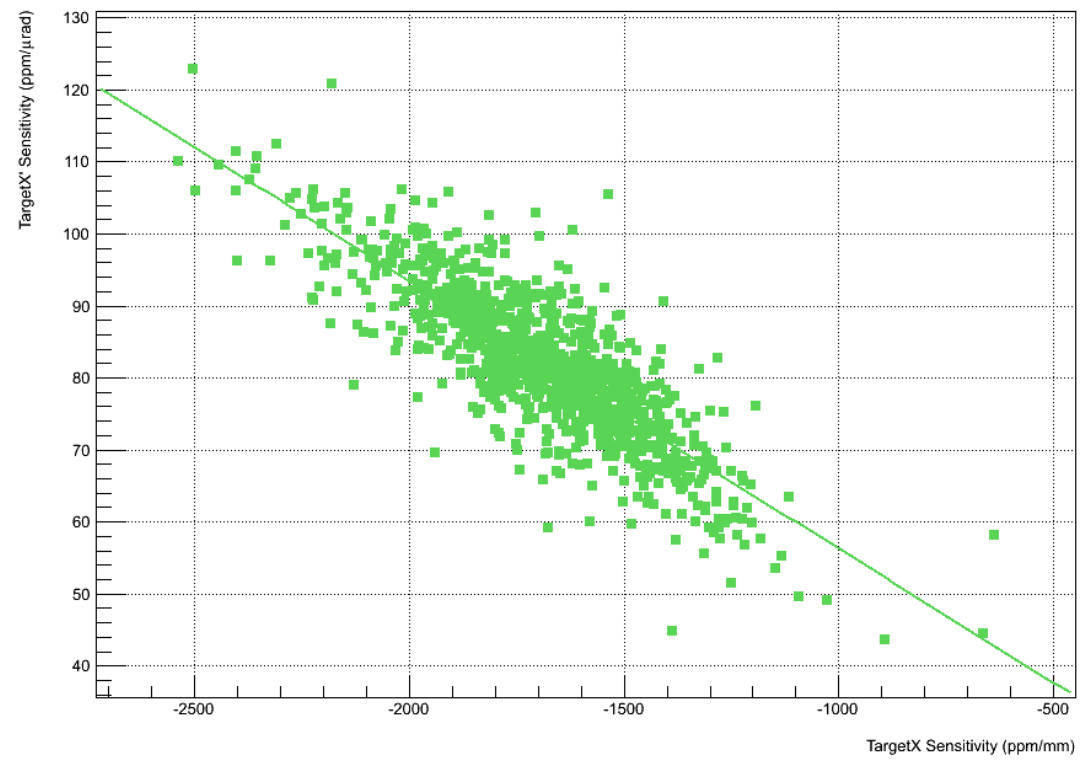

FIG. 4.8: Correlation between TargetX and TargetX' sensitivities.

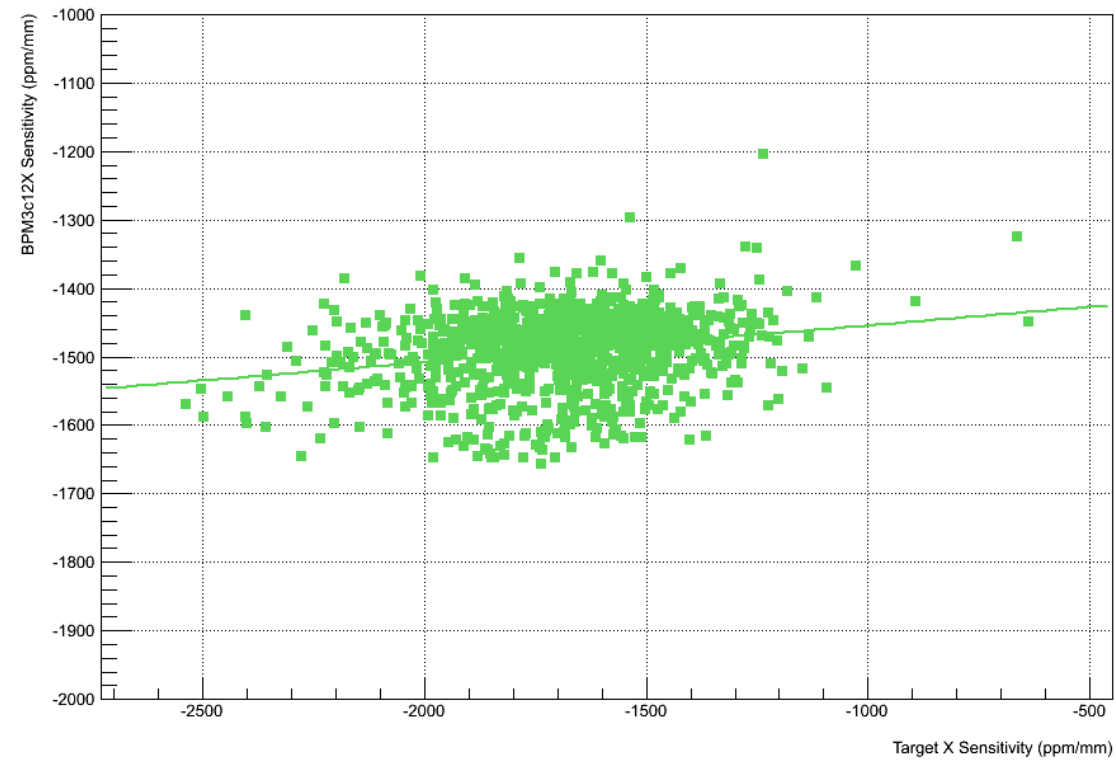

FIG. 4.9: Correlation between BPM3c12X and TargetX sensitivities. 


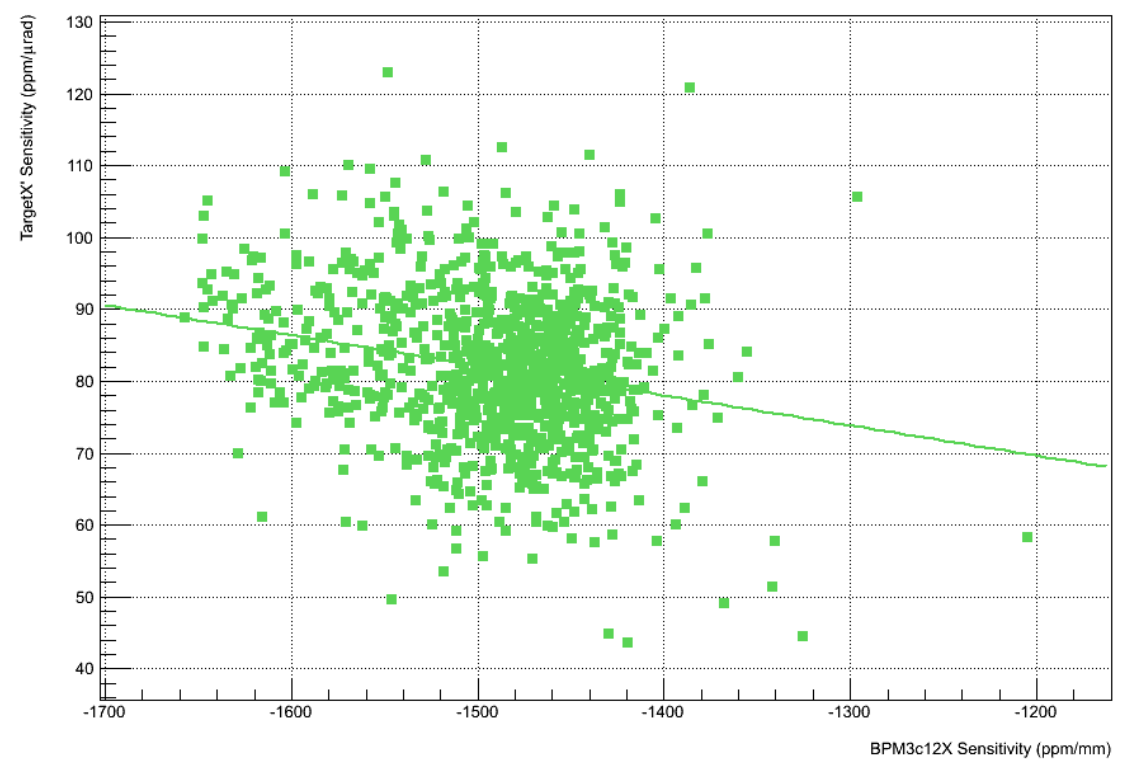

FIG. 4.10: Correlation between BPM3c12X and TargetX' sensitivities.

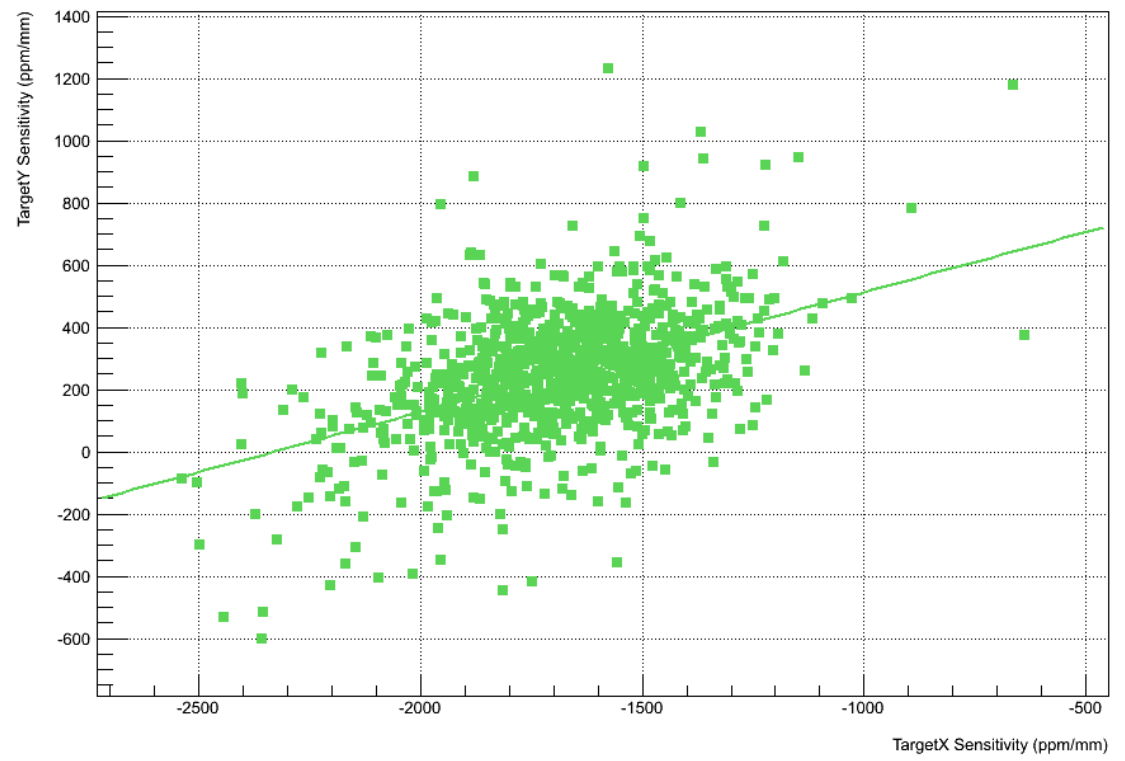

FIG. 4.11: Correlation between TargetY and TargetX sensitivities. 


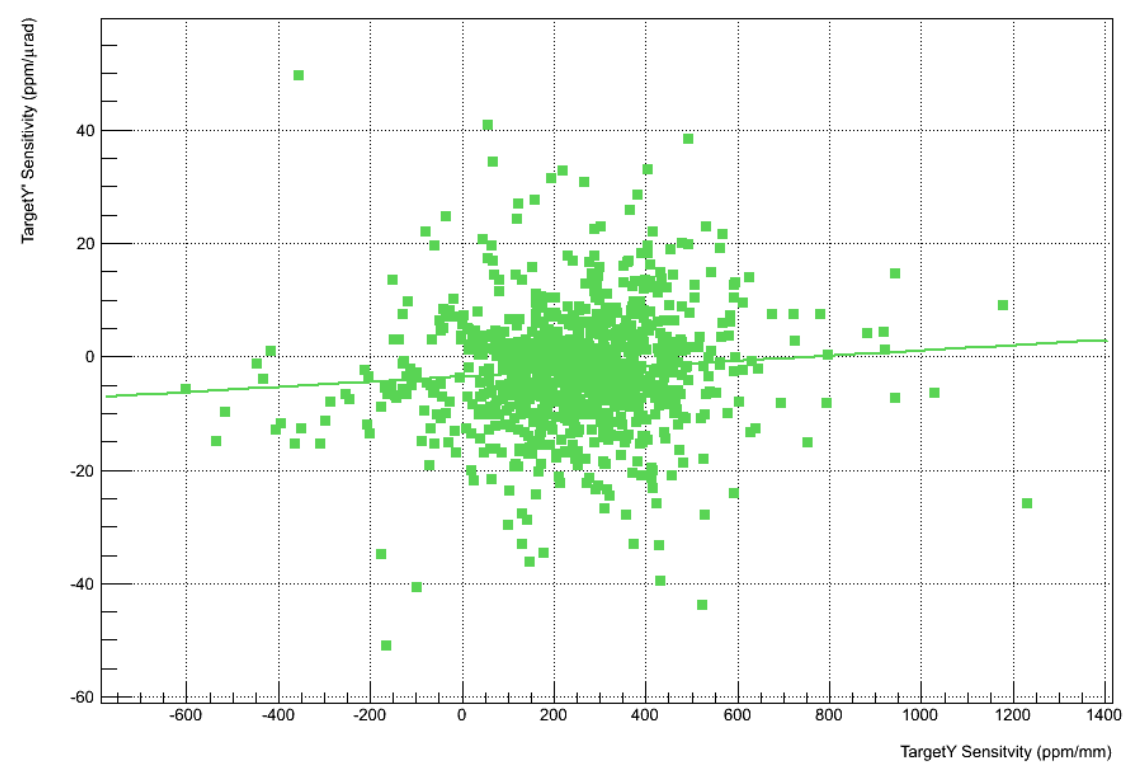

FIG. 4.12: Correlation between TargetY and Target $Y^{\prime}$ sensitivities.

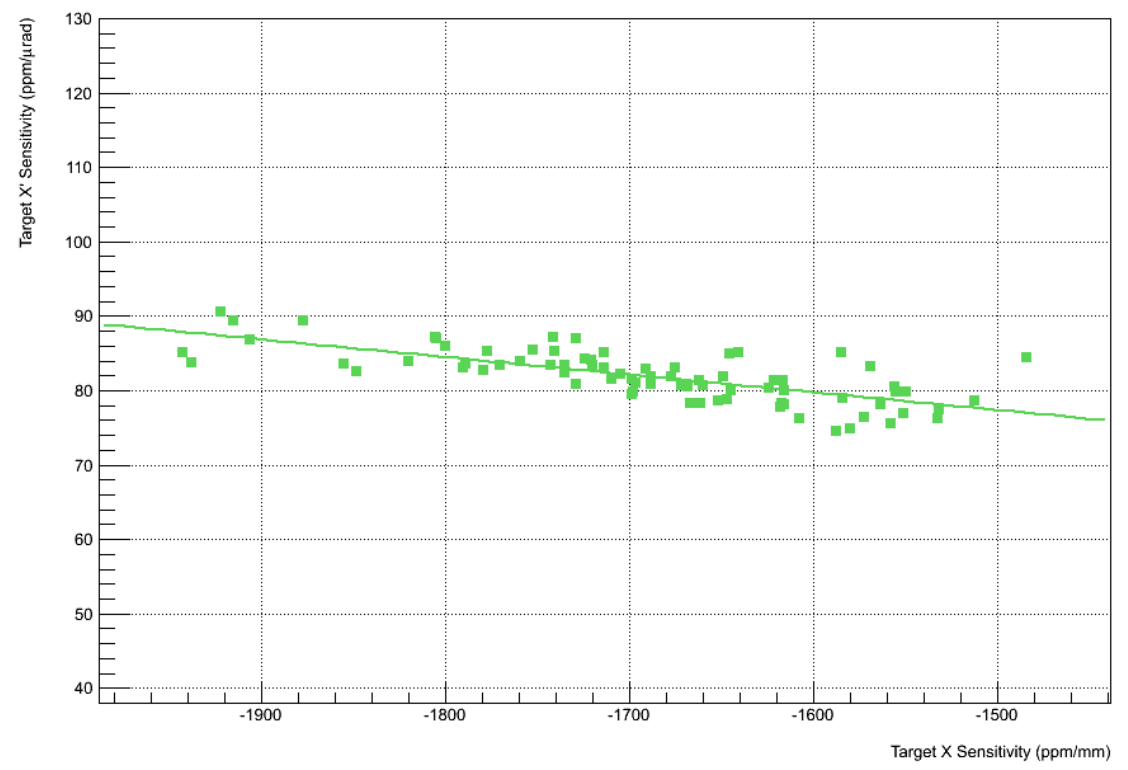

FIG. 4.13: Slug average correlation between TargetX and Target $\mathrm{X}^{\prime}$ sensitivities. 


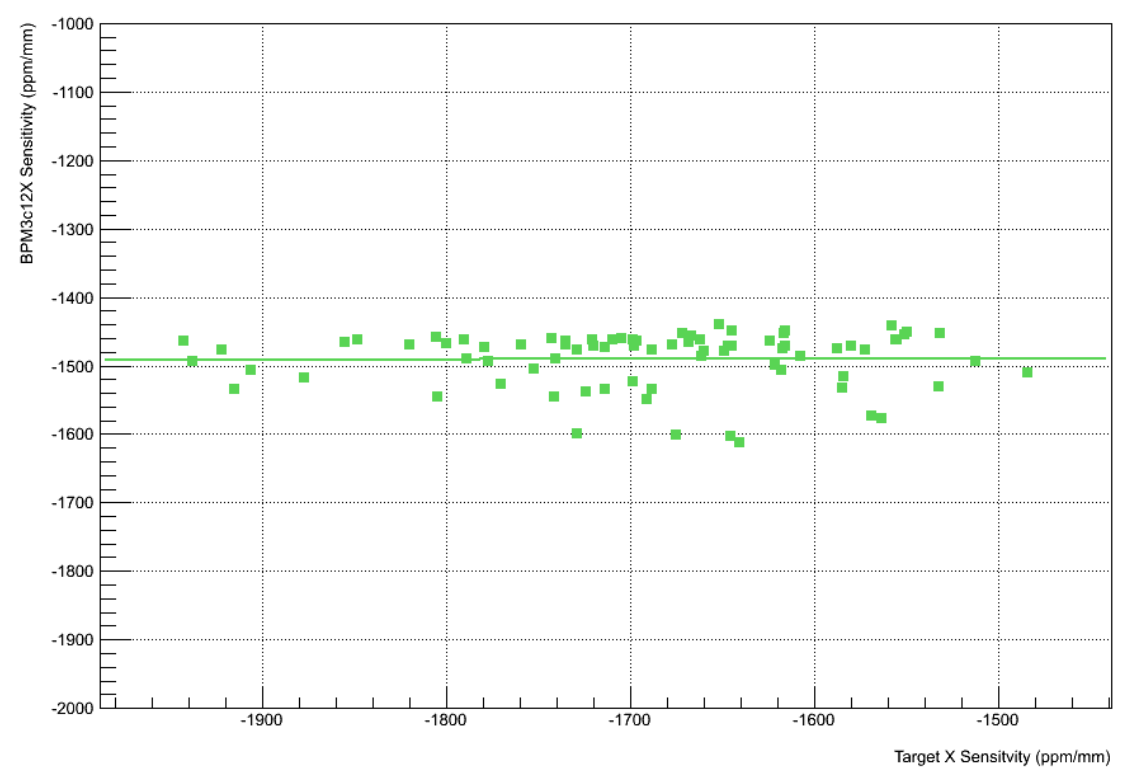

FIG. 4.14: Slug average correlation between BPM3c12X and TargetX sensitivities.

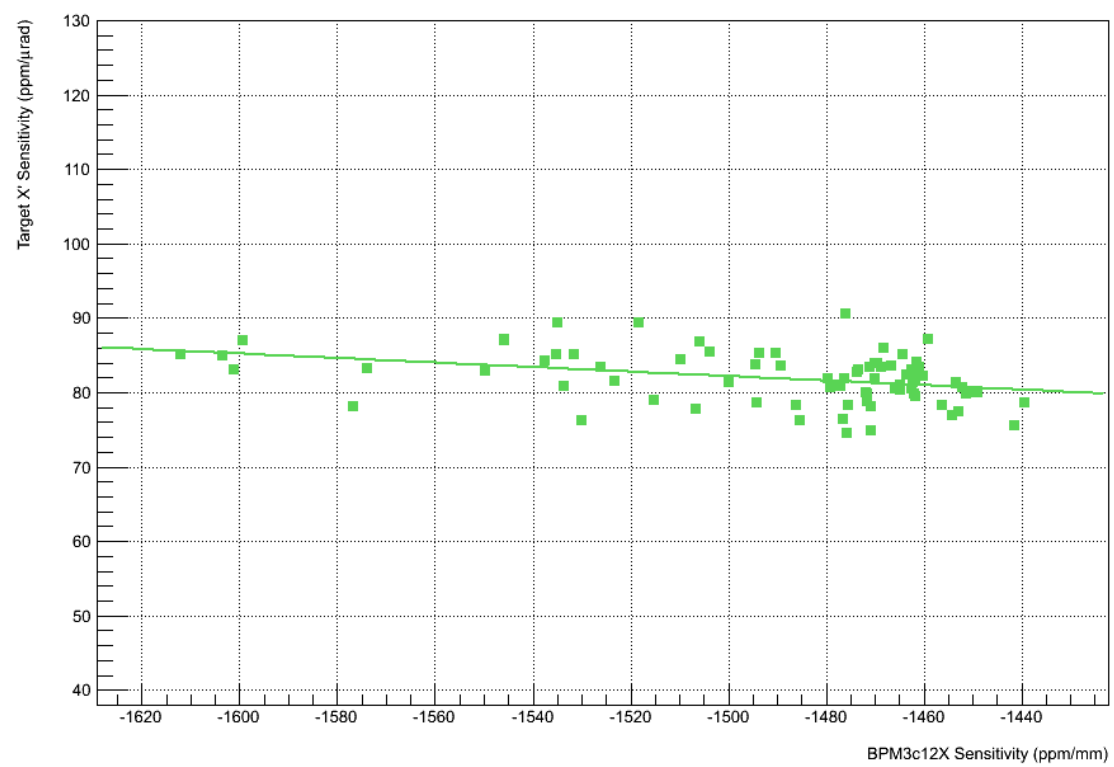

FIG. 4.15: Slug average correlation between BPM3c12X and TargetX' sensitivities. 


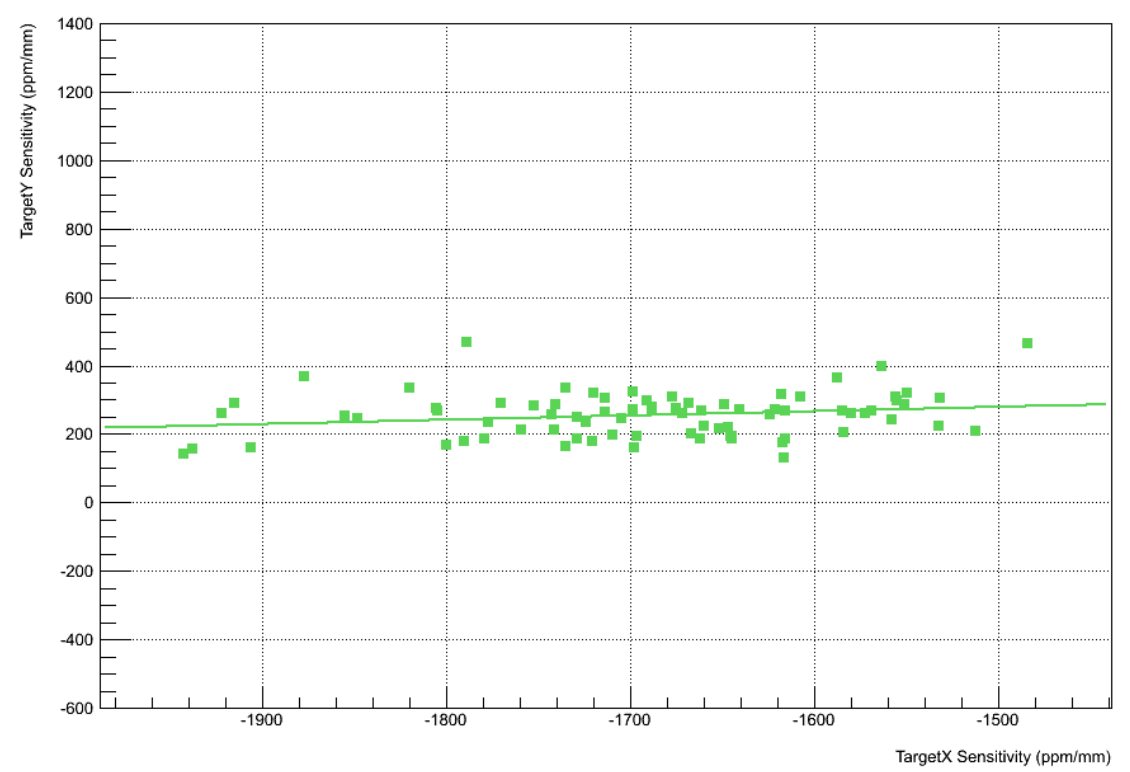

FIG. 4.16: Slug average correlation between TargetY and TargetX sensitivities.

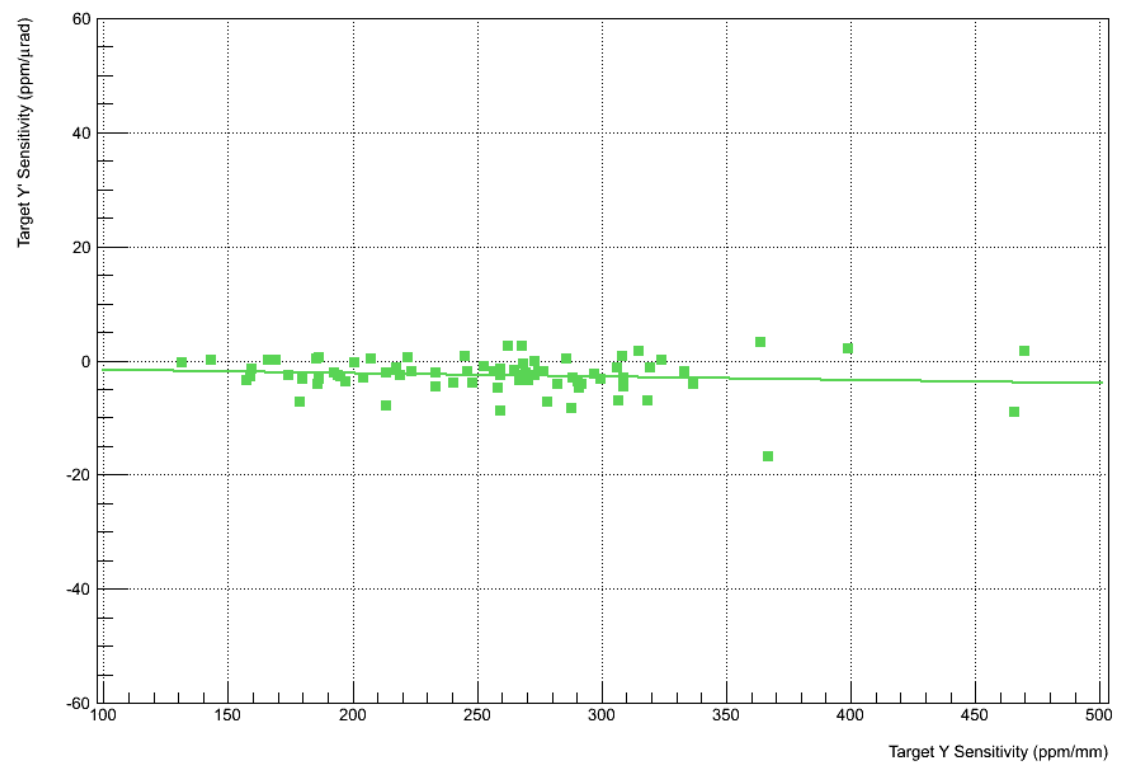

FIG. 4.17: Slug average correlation between TargetY and TargetX' $\mathrm{X}^{\prime}$ sensitivities.

During both Run I and Run II run periods data was taken with the FFB system 
deactivated. The original purpose of this, during Run I, was to study the effect of the FFB system on the response amplitude during modulation; if FFB significantly suppressed the BPM response amplitude we could not run it during modulation. The result of the Run I test was that the FFB reduced the BPM response $\sim 5 \%$ and there was a slight amplification of the Y response. Unfortunately, as mentioned previously, the phase shift due to FFB interacting with the modulation was overlooked. The response to modulation for each $\mathrm{BPM}$ during both runs with and without $\mathrm{FFB}$ active can be found in Fig. 4.18-4.22. From the plots the effect of the FFB on the BPM response is clear, with the effect on the Y-like BPMs being most significant. It is important to note that the phase shift of the BPM response during the FFB off run should be insignificant; in the case of energy modulation, because the energy lock is always disabled, there should be no phase shift in either case. The results for the FFB off run match expectations with the exception of Target $Y^{\prime}$ modulation. Referring to Fig. 4.22, the phase shift seen in Target $Y^{\prime}$ with FFB off is $\sim 70^{\circ}-80^{\circ}$. The fact that there is a significant phase shift in the BPM response to modulation when the FFB system is disabled, is suggestive of there being another set of coils coupling to the modulation. This was looked into more thoroughly by the beam corrections group at the University of Virginia; currently no source for this coupling has been found. One thing that group noticed was noticed was that the phase shift with FFB turned off is not present in the Run II results. The analysis to determine what the issue was is currently on-going, however, as mentioned above, the addition of an additional phase should not matter in the analysis as long as the shift does not change with time, however, it is still desirable to understand the source. 


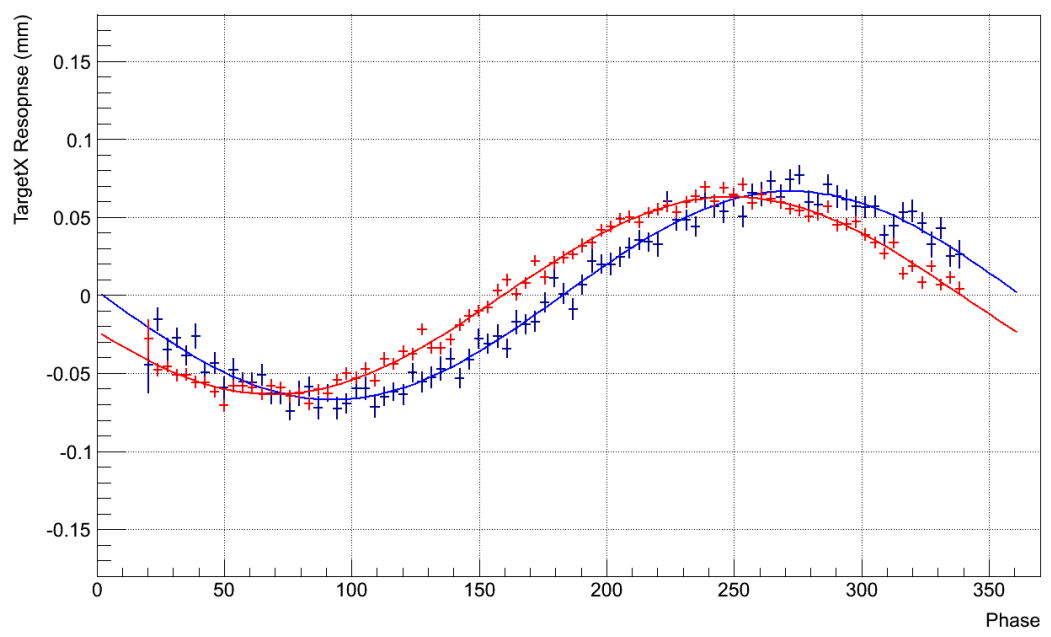

FIG. 4.18: TargetX response to modulation during runs with and without FFB active.

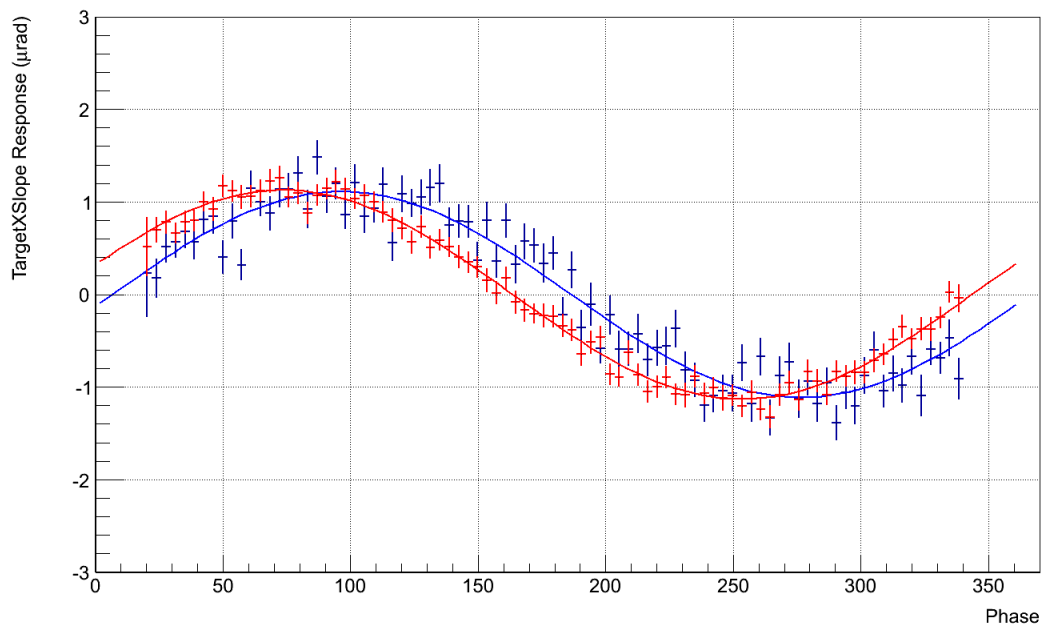

FIG. 4.19: Target $\mathrm{X}^{\prime}$ response to modulation during runs with and without FFB active. 


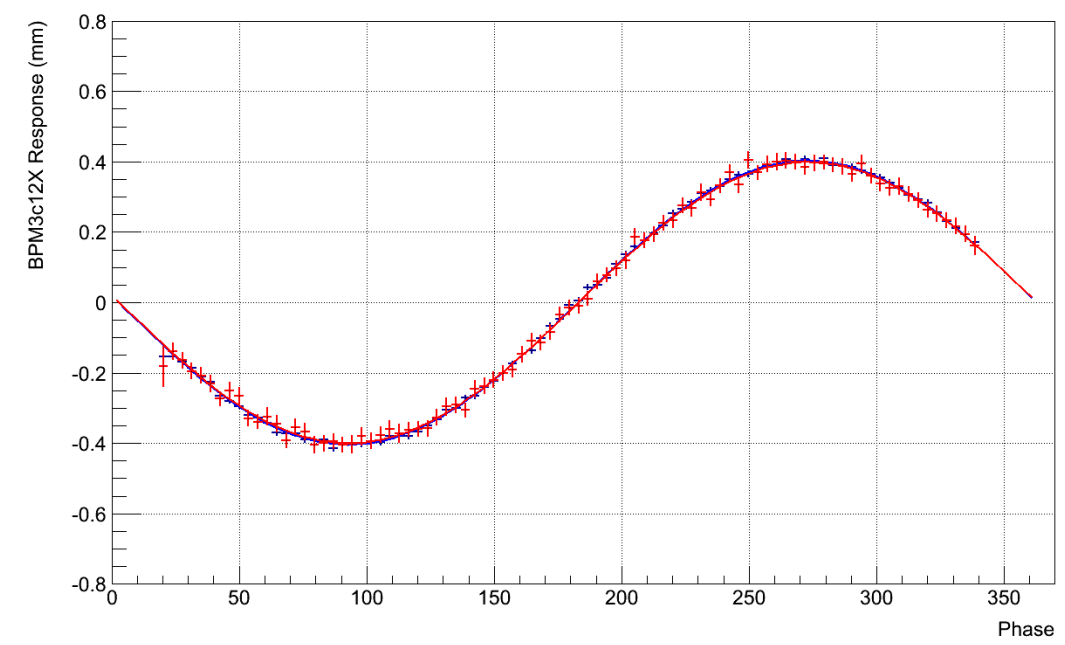

FIG. 4.20: BPM3c12X response to modulation during runs with and without FFB active.

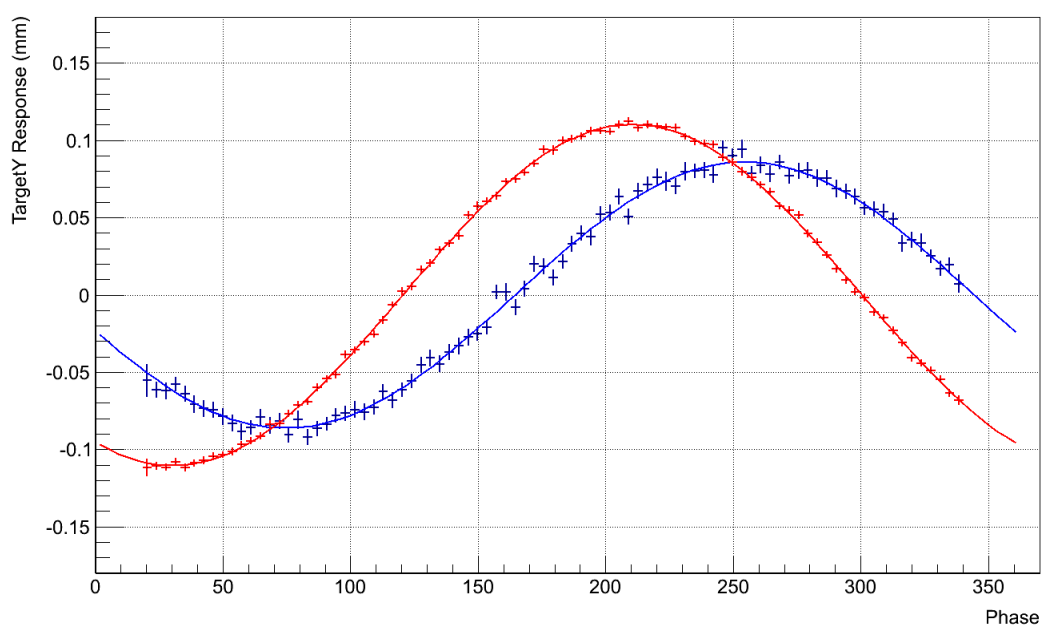

FIG. 4.21: TargetY response to modulation during runs with and without FFB active. 


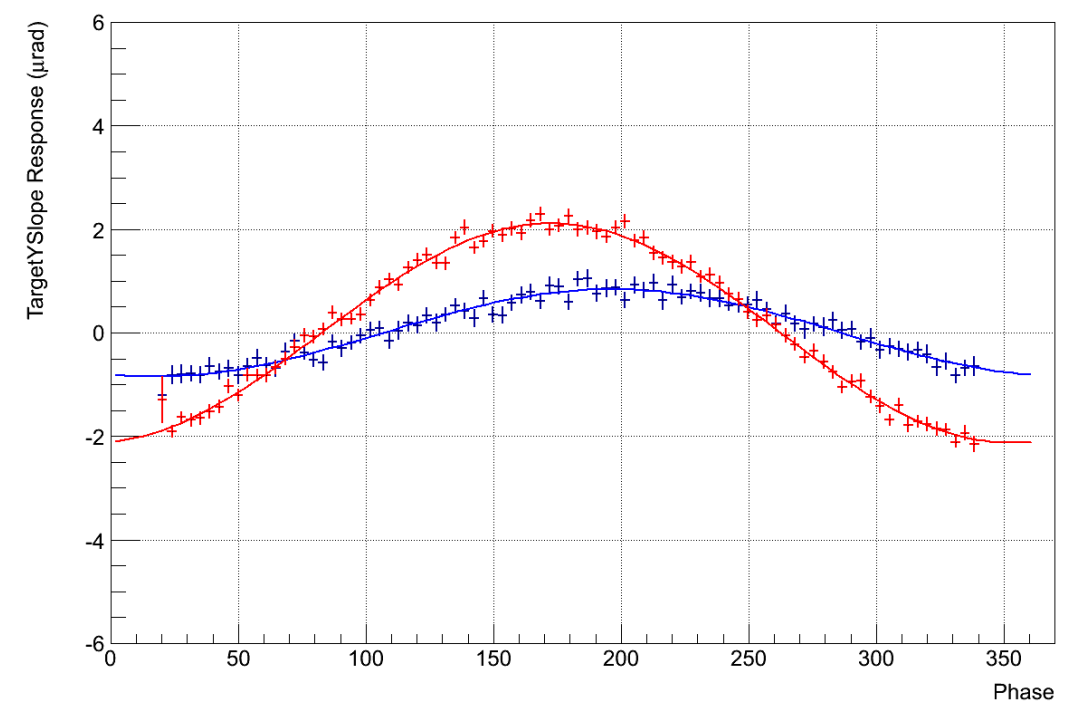

FIG. 4.22: TargetY' response to modulation during runs with and without FFB active.

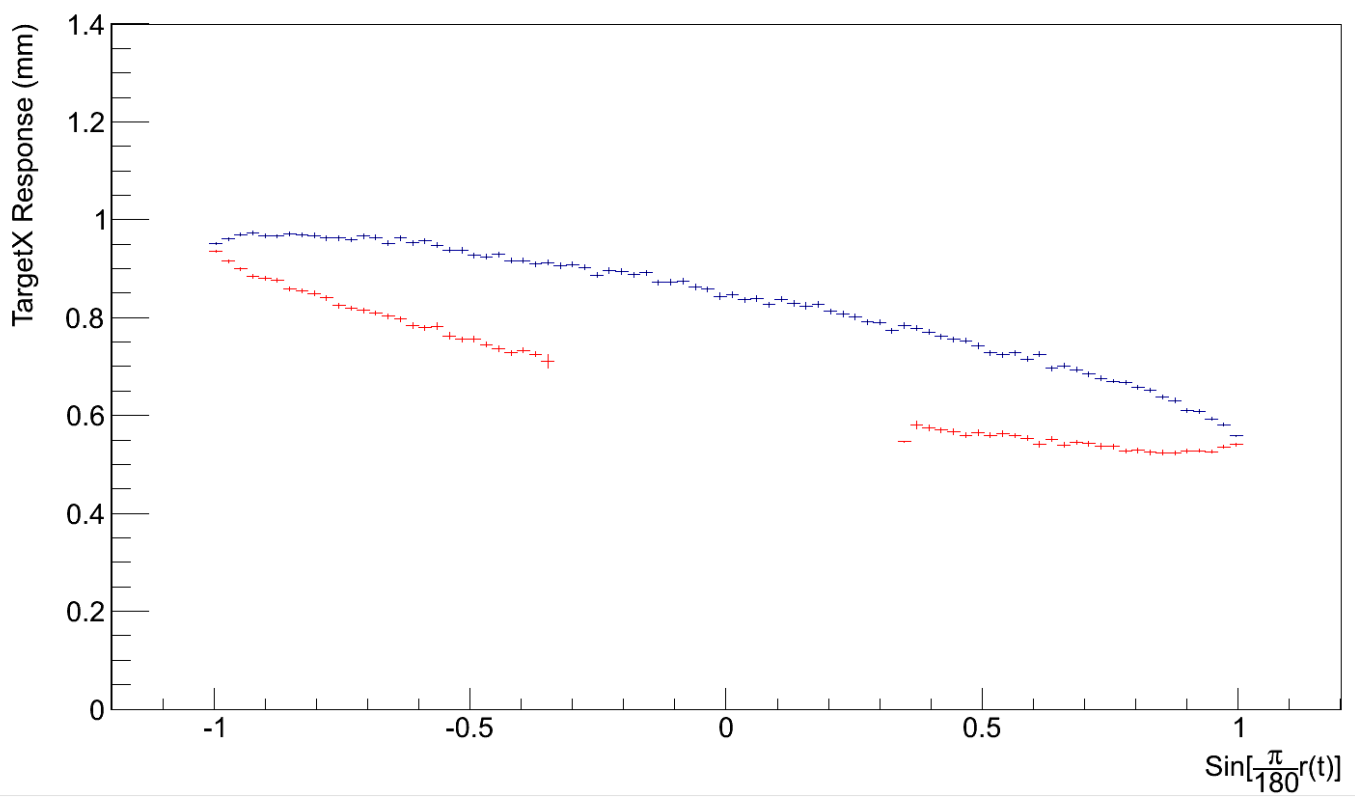

FIG. 4.24: The same as the above plot but profiled. The pull due to the cut ramp data is much more pronounced when looking at the average versus $\mathrm{R}(\mathrm{r}(\mathrm{t}))$.

One of the issues that arose from the BPM response phase shift was, when 


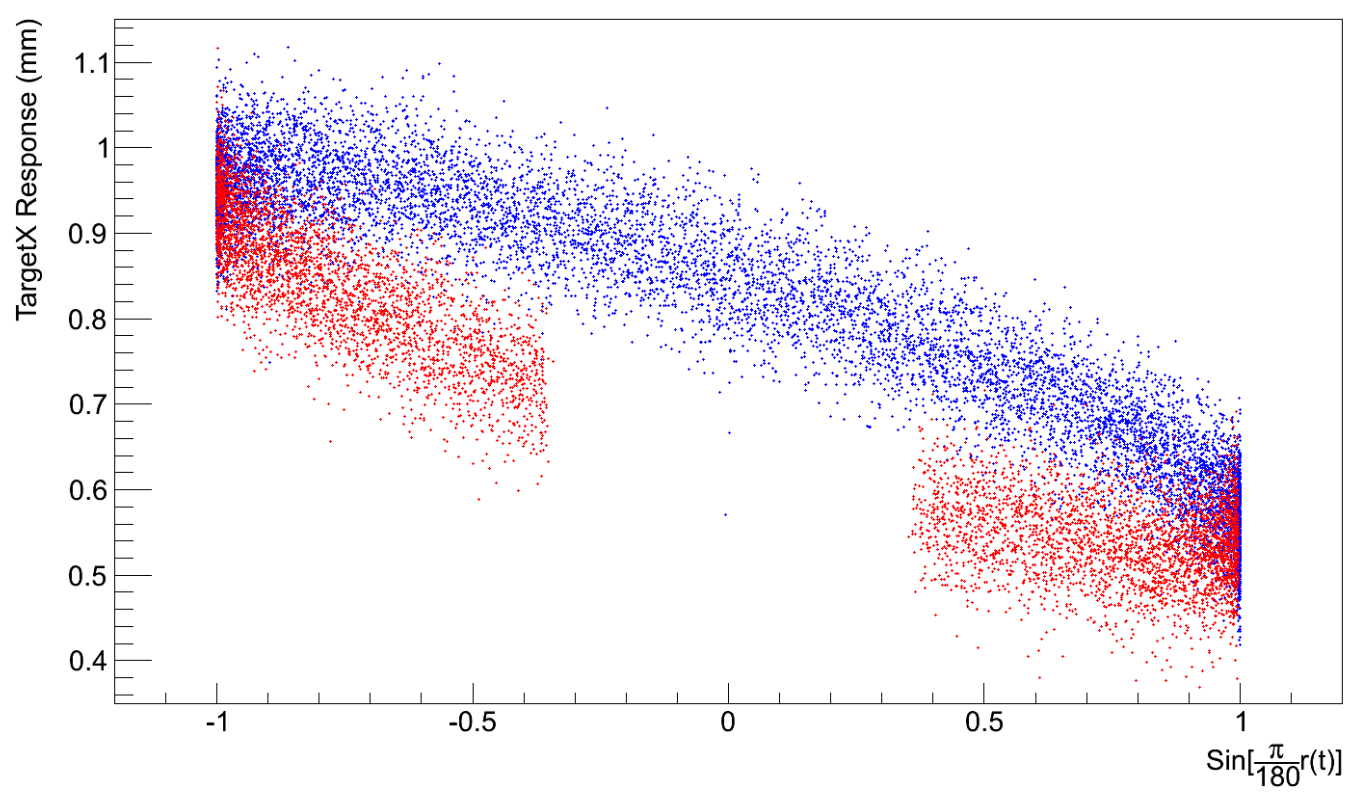

FIG. 4.23: The response of the TargetX BPM to $\mathrm{X}$ modulation plotted versus $R(r(t))=\sin \left(\frac{\pi}{180} r(t)+\phi\right)$, where $\mathrm{r}(\mathrm{t})$ is the ramp function. Because of the phase shift from the FFB system the response traces an ellipse in phase space; near the center of the ellipse the missing data due to the ramp cut can be seen. This missing data and the ellipsoid nature of the response-induced extra error into the sensitivities extraction and made analytical calculation of the errors on the sensitivities more difficult.

looking at the correlation between the BPM response and the sine of the ramp function (recall Eq. 4.8), that the result was not linear. Looking at the plot of the TargetX virtual BPM versus the sine of the ramp (Fig. 4.23) - defined as $R(r(t))=\sin \left(\frac{\pi}{180} r(t)+\phi\right)$, where $r(t)$ is the ramp function - you can see that the result traces out an ellipse in phase space. This causes problems in two ways. First, because the ramp cut removes events calculated incorrectly during the transition period of the ramp function, the ellipsoid is not filled continuously in phase space, i.e. the ellipse is double valued in places and single valued in others. Fig. 4.23 shows a scatter plot of TargetX versus $\mathrm{R}(\mathrm{r}(\mathrm{t}))$ and Fig. 4.24 shows a profiled version of the same plot. The data removed by the ramp cut causes the response ellipse (4.23) to 
be non-continuous, potentially causing the calculation of the correlation performed in Eq. 4.8 to be incorrect. The second issue, which would be present even if the ramp cut was not applied, is that the error on the correlation calculation will be incorrect; this is because we are applying a linear model to a non-linear response.

Following the completion of the modulation analysis replay, the effect of the missing ramp data was investigated. A method to fill in the missing ramp data and make the response continuous was developed at the University of Virginia; this method of filling in the missing ramp data would have required a new full replay of the data and was not used in the present analysis, however, early results using the ramp fill method were compared [69] to the results found here and showed only a small change. Given the difficulties in computing the errors on each extracted sensitivity explained above, the errors were instead extracted by looking at the $R M S / \sqrt{N}$ for each sensitivity on the Slug and Wien level. Shifting the analysis to the Slug and Wien levels not only allowed for the extraction of more accurate error bars, but Slug or Wien level sensitivities facilitated the use of more accurate detector sensitivities; the sensitivity of the detectors to changes in the beam parameters was a property of the apparatus and should have been constant. These Slug or Wien level sensitivities can then be applied to the data for more accurate corrections.

Looking at the single detector sensitivities as a function of octant number provides an important diagnostic tool as well as providing insight into the nature of the detector sensitivities. In Fig. 4.25 - 4.27 the Run I average single detector sensitivities are shown as a function of octant number. Recall from Sec. 3.5.4, the main detector is an azimuthally symmetric array of quartz Čerenkov detectors. The octants are numbered as shown in Fig. 3.12, with octants 1 and 5 in the horizontal plane. With these definitions, it is expected that the measured detector sensitivity for each bar will be characteristic of the modulation type. 
For instance, for an X-like modulation the single detector sensitivity as a function of octant number should be sinusoidally varying with peaks at octant 1 and octant 5; these octants are in the plane of modulation and should be most sensitive. In Fig. 4.25 a plot of the Wien-average single detector X-sensitivity as a function of octant number is shown. The detectors in the plane of modulation show the greatest sensitivity to X-like modulation. Taking note of the size of the largest single detector sensitivities, which are on the $\mathcal{O}( \pm 5500 \mathrm{ppm} / \mathrm{mm})$, we get a factor of $\sim 3$ suppression in the octant-average value due to the detector symmetry; the suppression in $\mathrm{Y}$ is much greater, $\sim 24$. The size of the detector averaged sensitivities could be attributed to some degree to the beam not being centred on the neutral axis of the detector; if the parts of the scattered electron profile fall off the detector bar it would increase the position sensitivity. Studies of the relative main detector widths [70] do suggest that our choice of average beam position was not optimal. Another likely source was background caused by beam halo. Beam halo is a low-density collection of particles that gather around the core of the electron beam. Halo can be caused by a number of things including: single and multi-particle scattering, machine non-linearities, and noise in the injector. During beam modulation the beam halo can interact with elements along the beam line causing backgrounds which could manifest as a monopole in the detectors. 


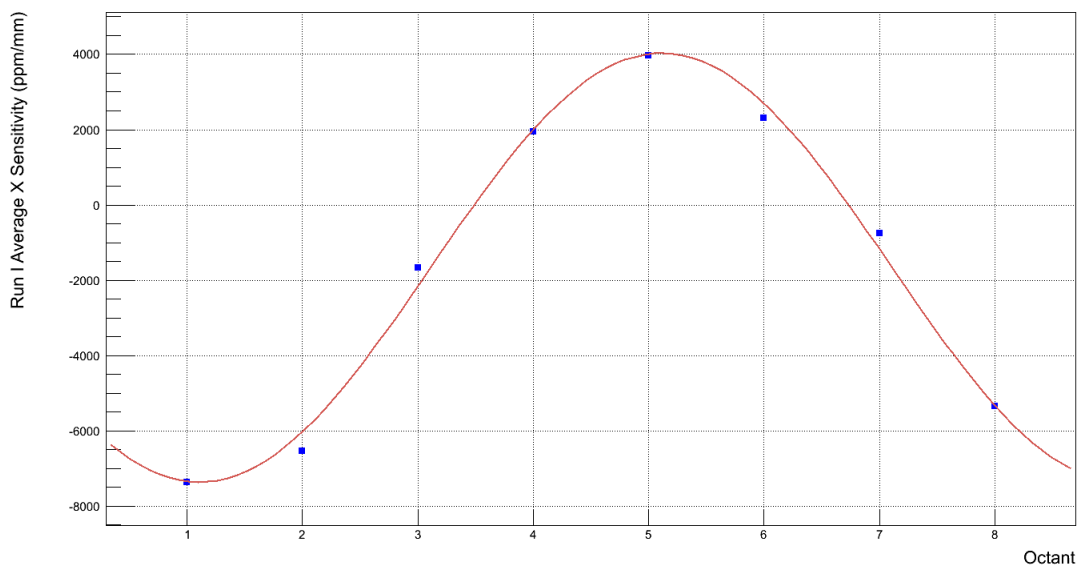

FIG. 4.25: The Run I single detector X position sensitivities over Run I versus octant number for $\mathrm{X}$ modulation. Because $\mathrm{X}$ modulation is in the horizontal plane, detectors 1 and 5 are most sensitive to this modulation type. The sinusoidal variance of the detector sensitivities, with peaks at 1 and 5 , is characteristic of X-like modulation. Error bars are included but are smaller than the markers.

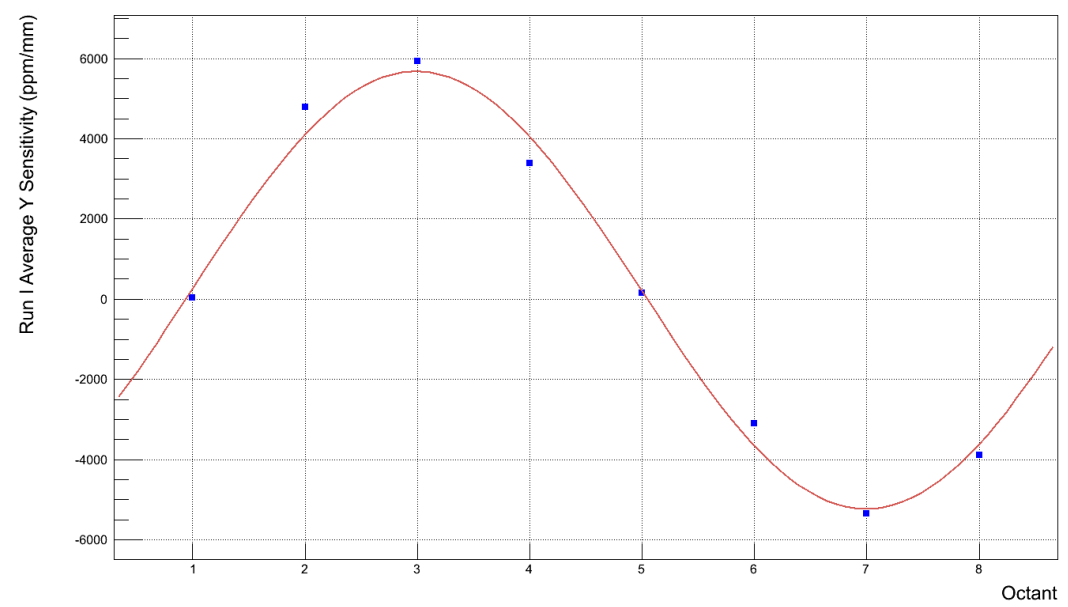

FIG. 4.26: The Run I single detector $\mathrm{Y}$ position sensitivities over Run I versus octant number for $\mathrm{X}$ modulation. Because $\mathrm{Y}$ modulation is in the vertical plane, detectors 3 and 7 are most sensitive to this modulation type. Error bars are included but are smaller than the markers. 


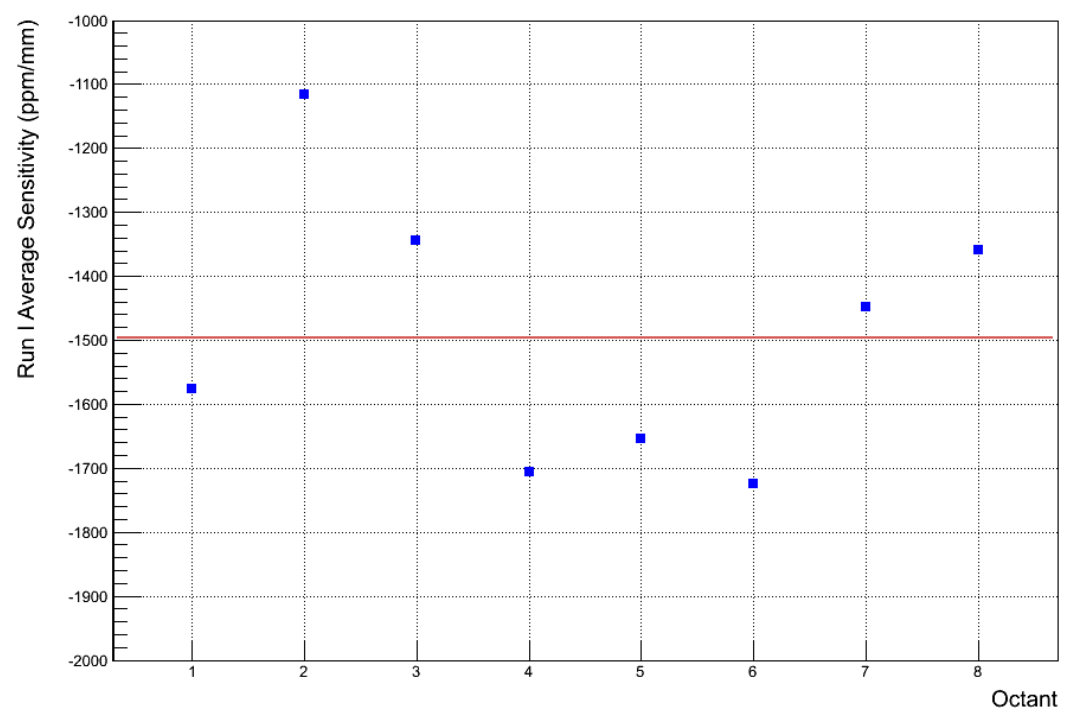

FIG. 4.27: The Run I single detector energy sensitivities over Run I versus octant number for E modulation. Ideally, a change in energy should be seen as a change in $\mathrm{Q}^{2}$ and effect each detector uniformly. The expected response would be a detector monopole. Error bars are included but are smaller than the markers.

The extracted detector sensitivities were studied at the runlet scale - "runlet" for modulation data is a different unit than mentioned above and corresponds to approximately half a full run - slug, and Wien levels. The detector sensitivities, if properly determined, should be a property of the detector apparatus and therefore should be static. Studying the detector sensitivities at longer time scales allows us to reduce the statistical noise and determine both the stability of the extracted sensitivities and the time scale on which it is most appropriate to apply corrections to the asymmetry. Shown in Fig. 4.28 - 4.32 are the Slug-average detector sensitivities for Run I, weighted by the main detector error. The extracted detector sensitivities for each beam parameter on the Wien level are shown in Table 4.7. Aside from the occasional outlier, the Slug-level sensitivities are generally stable. One aspect where there does seem to be a shift in the sensitivities is bpm3c12X; starting after Slug 
100 the sensitivity starts to drift.

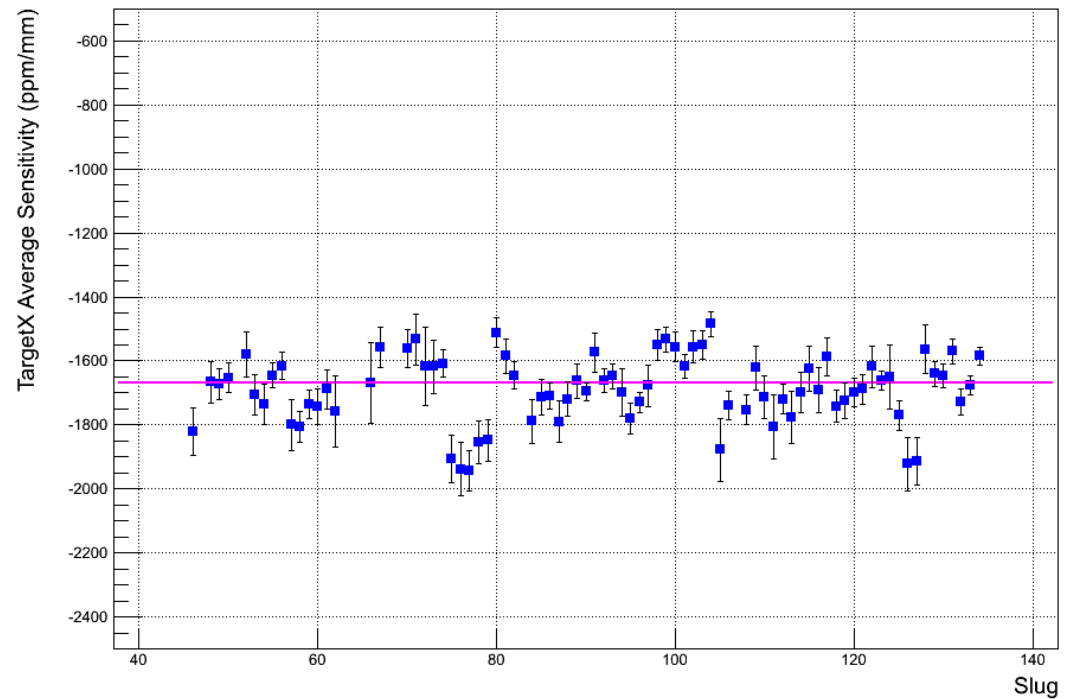

FIG. 4.28: Run I Slug-average Set 1 X sensitivities.

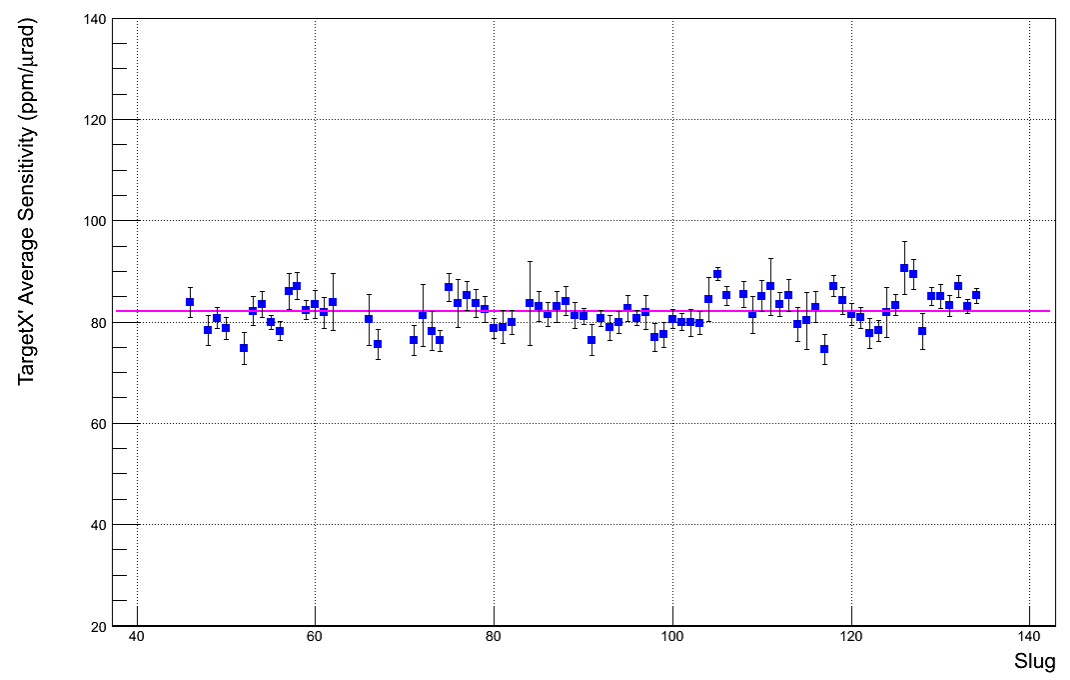

FIG. 4.29: Run I Slug-average Set $1 \mathrm{X}^{\prime}$ sensitivities. 


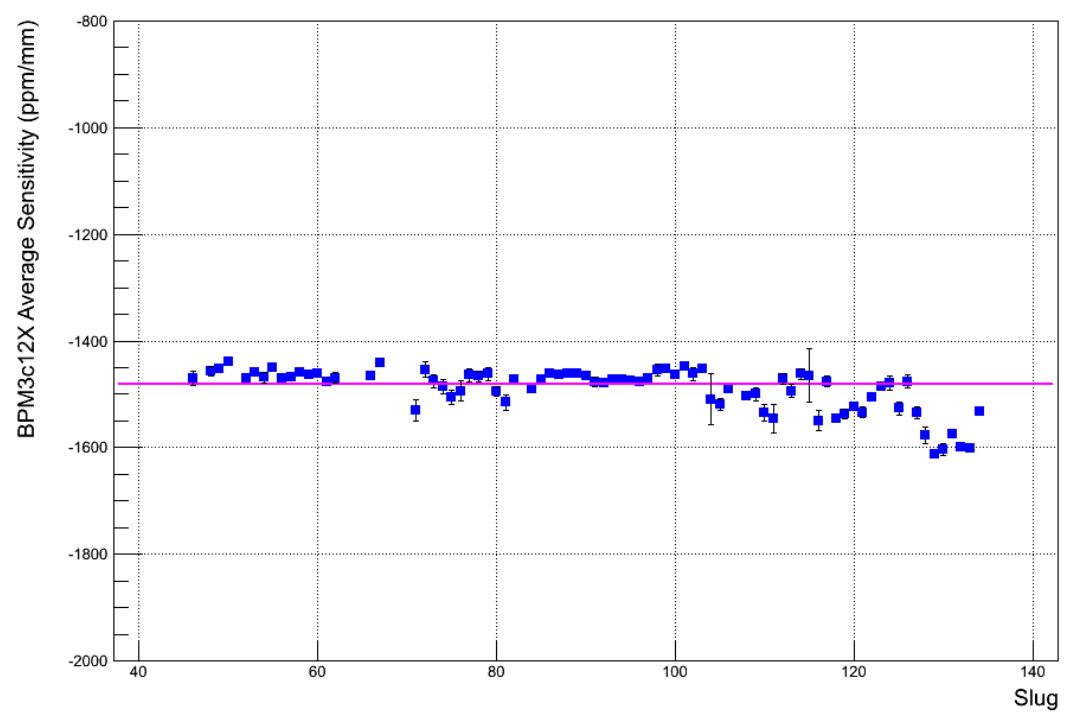

FIG. 4.30: Run I Slug-average Set 1 BPM3c12X sensitivities.

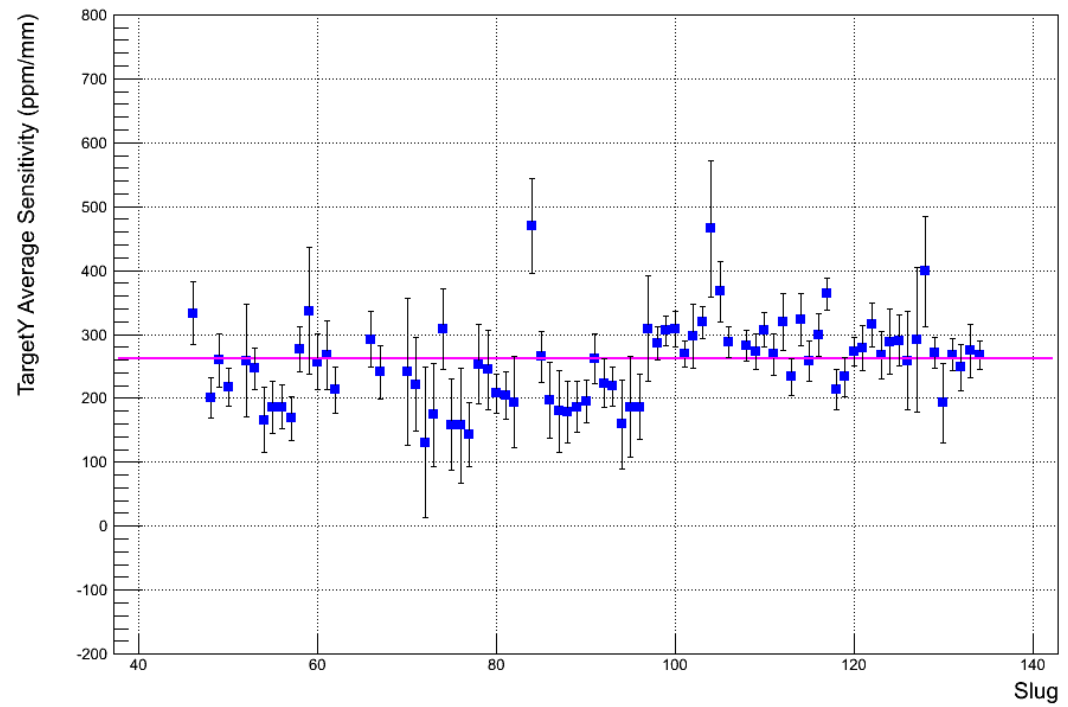

FIG. 4.31: Run I Slug-average Set 1 Y sensitivities. 


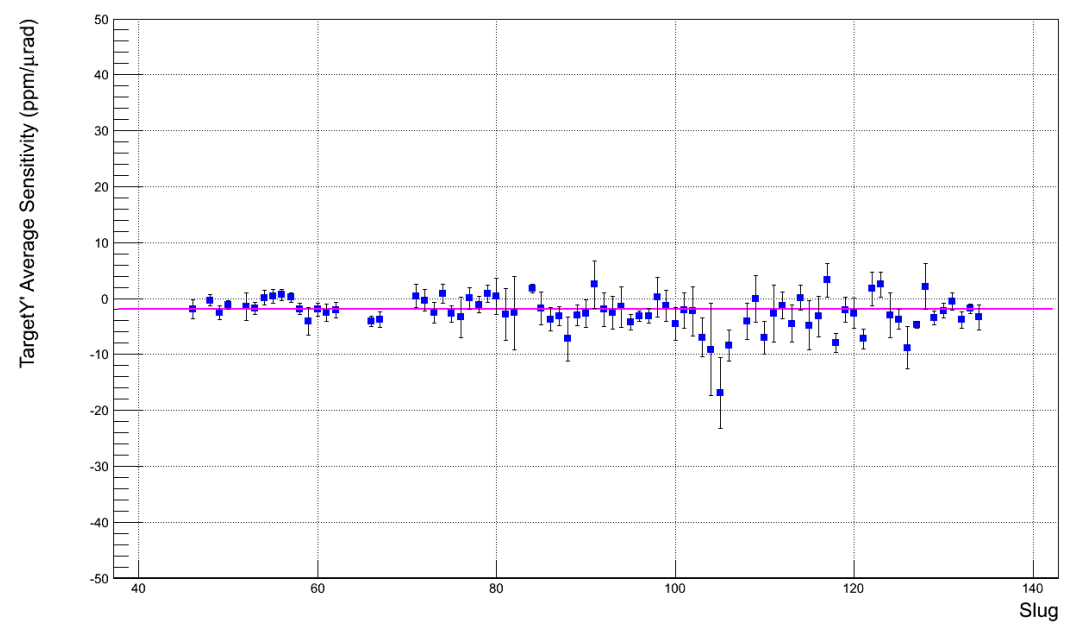

FIG. 4.32: Run I Slug-average Set $1 Y^{\prime}$ sensitivities.

A suitable metric for whether the modulation set results agree between phase sets is the Wien-level total corrections to the asymmetry for each set. In Table 4.4 the total correction for each Wien and each set is shown. It should be noted that because the position differences are different from Wien-to-Wien there is no reason for the corrections to be the same for different Wiens. From the table it can be seen that, within errors, the corrections are consistent between sets. This shows that the choice of phase, while changing the strength sharing, does not matter at the current level of precision in extraction of the sensitivities. For the analysis presented in this thesis, Set 1 was chosen. This choice was dictated by the simplicity of having no phase shift and by the size of the extracted errors on the sensitivities.

One of the issues with determining the quality of the beam modulation sensitivities comes from the fact that the data used to extract the detector sensitivities is not the same data that is corrected; this is not a problem for the standard linear regression of natural beam motion as it uses identical data sets. If we are correctly extracting the detector sensitivities and they are not changing there should be no 


\begin{tabular}{|l|r|r|r|r|}
\hline Wien/Set & Set $1(\mathrm{ppb})$ & Set 2 $(\mathrm{ppb})$ & Set 3 $(\mathrm{ppb})$ & Set $4(\mathrm{ppb})$ \\
\hline Wien 1 & $-15.9 \pm 7.2$ & $-12.8 \pm 7.4$ & $-15.9 \pm 7.1$ & $-11.2 \pm 6.5$ \\
\hline Wien 2 & $10.0 \pm 4.3$ & $5.4 \pm 4.4$ & $10.1 \pm 4.3$ & $14.4 \pm 3.9$ \\
\hline Wien 3 & $-7.7 \pm 5.5$ & $-7.8 \pm 5.5$ & $-7.5 \pm 5.5$ & $-10.9 \pm 5.1$ \\
\hline Wien 4 & $-26.8 \pm 5.4$ & $-27.9 \pm 5.3$ & $-26.9 \pm 5.4$ & $-27.3 \pm 5.0$ \\
\hline Wien 5 & $-17.6 \pm 6.6$ & $-18.9 \pm 6.5$ & $-17.6 \pm 6.7$ & $-16.7 \pm 6.1$ \\
\hline
\end{tabular}

TABLE 4.4: Wien level total corrections for each modulation Set.

problem correcting non-modulation data using beam modulation data, however as a proof-of-principle diagnostic it is preferable to first look at a more straightforward problem. The simplest diagnostic available is to confirm that the extracted detector sensitivities effectively correct the beam modulation yield response from which they were extracted. The yield response to modulation was corrected according to,

$$
Y_{\text {corrected }}=Y_{\text {raw }}-\sum_{i=1}^{5} \frac{1}{\langle Y\rangle}\left\langle\frac{\partial Y}{\partial X_{i}}\right\rangle(X-\langle X\rangle) .
$$

$Y_{\text {raw }}$ is the uncorrected detector yield response to modulation of a given modulation type, and $\left\langle\frac{\partial Y}{\partial X_{i}}\right\rangle$ are the Wien-average detector sensitivities. The yield response study was done using the Set 1 Wien-average detector sensitivities to correct the Run I data set. Before and after correction, the Wien-average yield response for each detector was plotted versus octant for both sine and cosine components. The sine(cosine) components for each detector were extracted by fitting to a composite equation of the form,

$$
Y(\theta)=Y_{\circ}+A_{\sin } \sin \left(\alpha x_{o c t}+\phi_{\text {set }}\right)+A_{\cos } \cos \left(\alpha x_{o c t}+\phi_{\text {set }}\right) .
$$

Here $\phi_{\text {set }}$ represents the phase setting for the modulation set that is used; in the case of this study this was Set $1(\phi=0)$. The results of the uncorrected yield response to modulation can be seen in Fig. 4.33 - 4.37. In these plots the results 
from the composite fit in Eq. 4.16 can be seen for each detector fitted to a simple sine function with a floating phase.

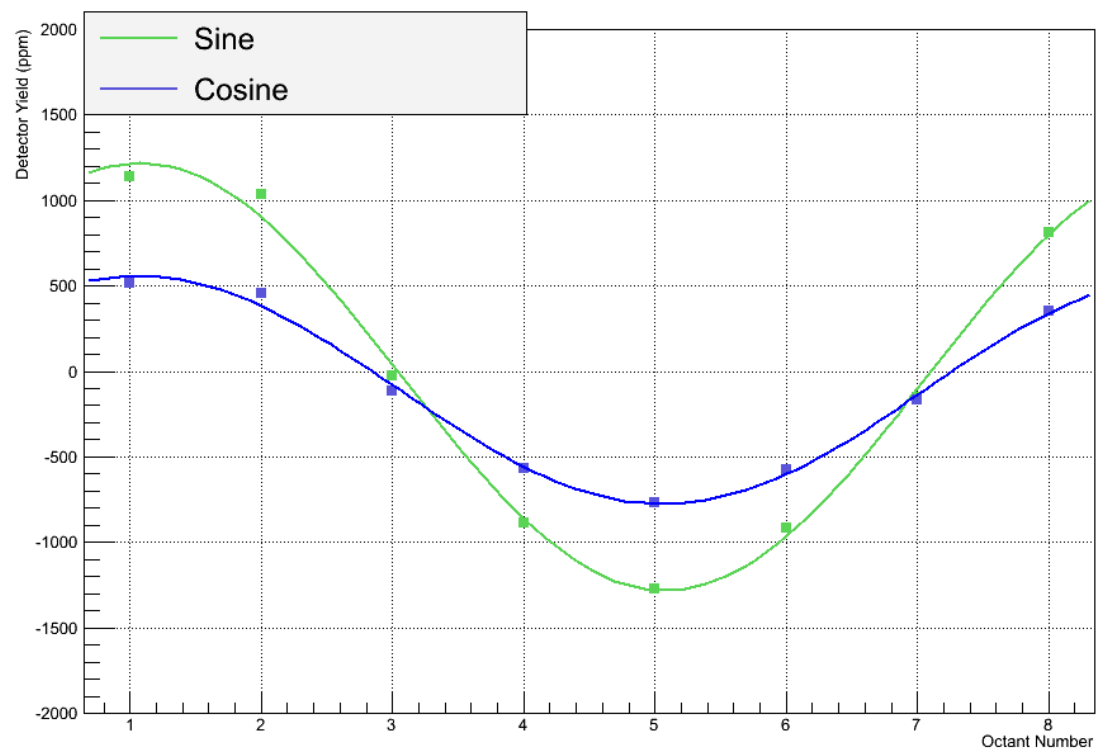

FIG. 4.33: Uncorrected yield response dipole to X modulation.

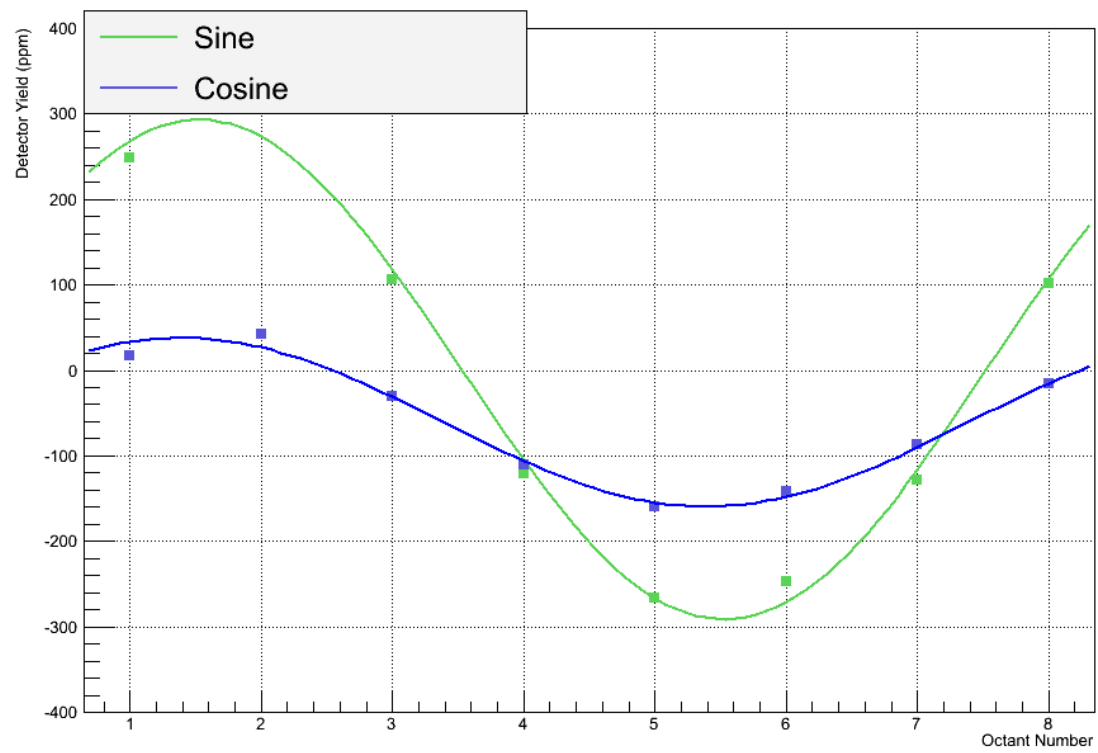

FIG. 4.34: Uncorrected yield response dipole to $\mathrm{X}^{\prime}$ modulation. 


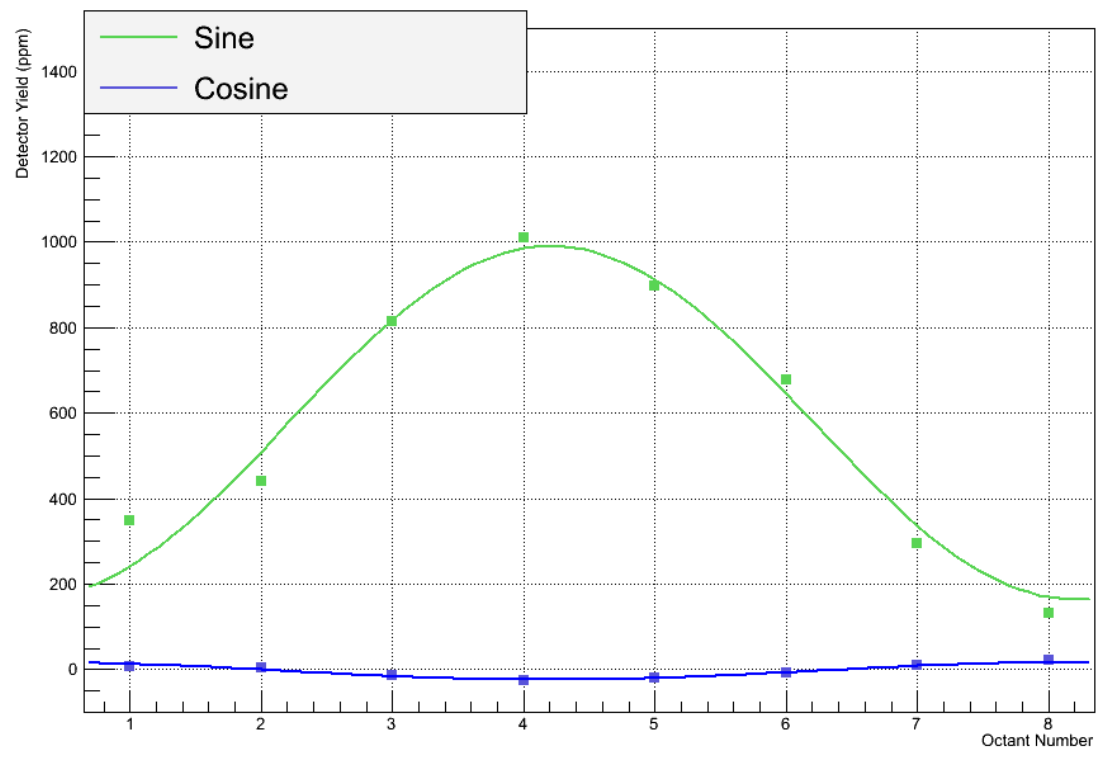

FIG. 4.35: Uncorrected yield response dipole to E modulation.

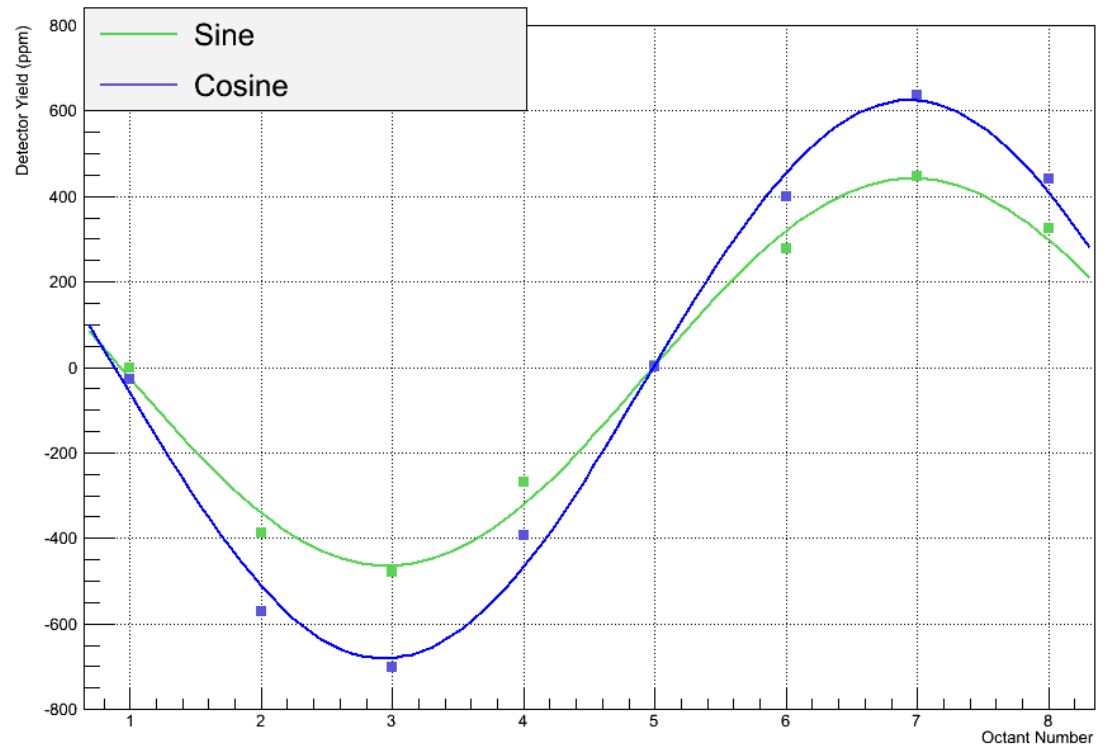

FIG. 4.36: Uncorrected yield response dipole to Y modulation. 


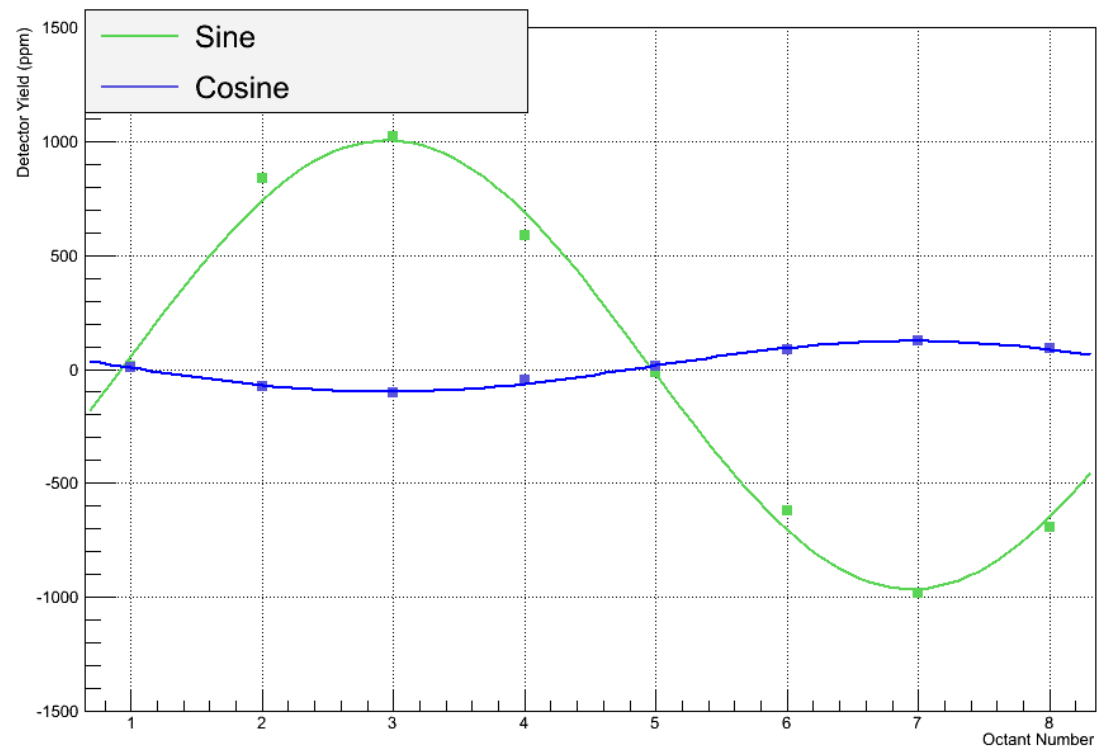

FIG. 4.37: Uncorrected yield response dipole to YP modulation.

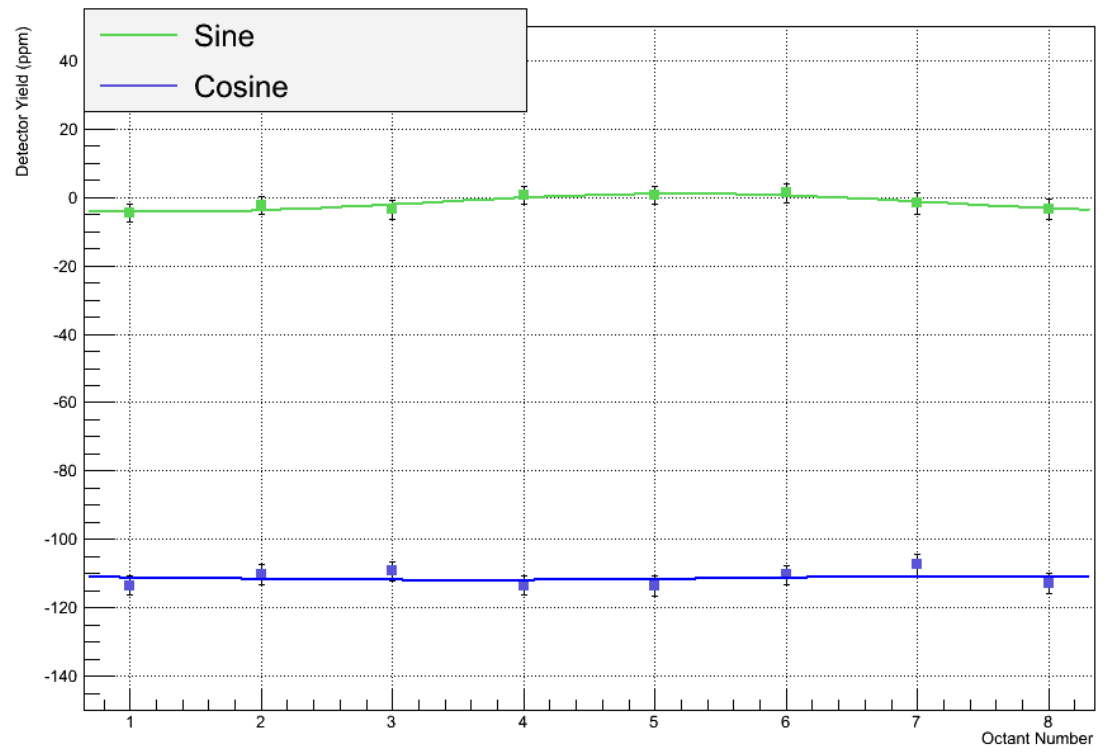

FIG. 4.38: Corrected yield response dipole to X modulation. 


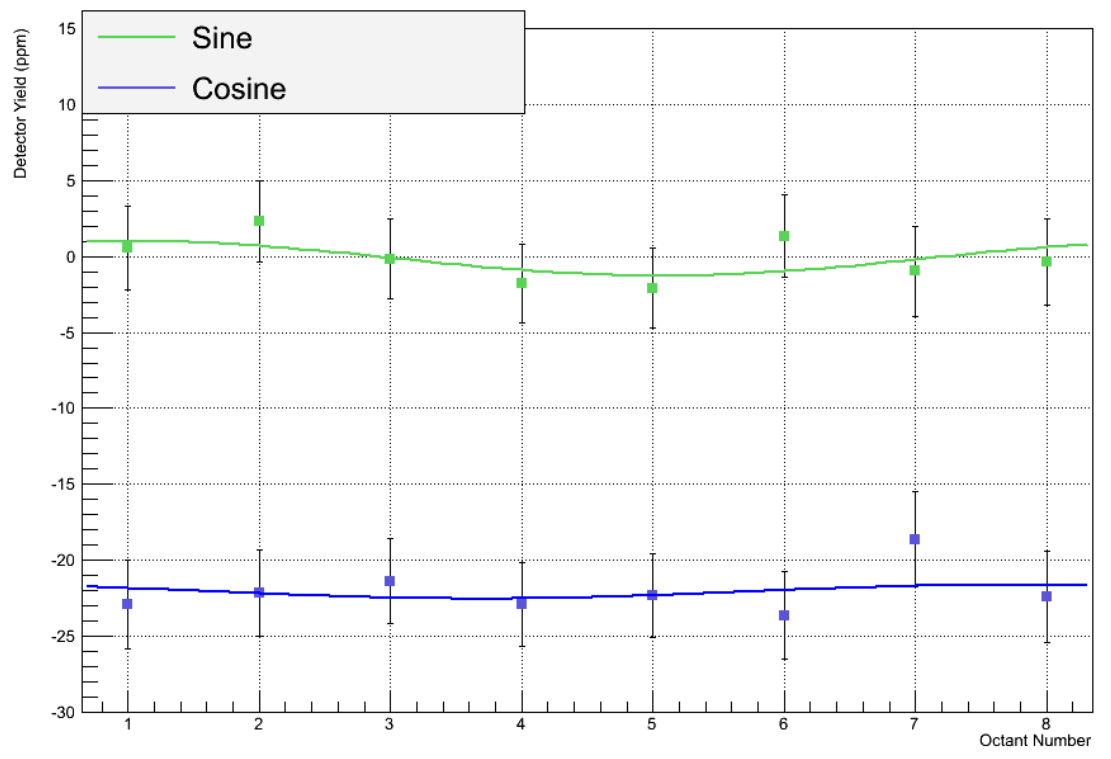

FIG. 4.39: Corrected yield response dipole to $\mathrm{X}^{\prime}$ modulation.

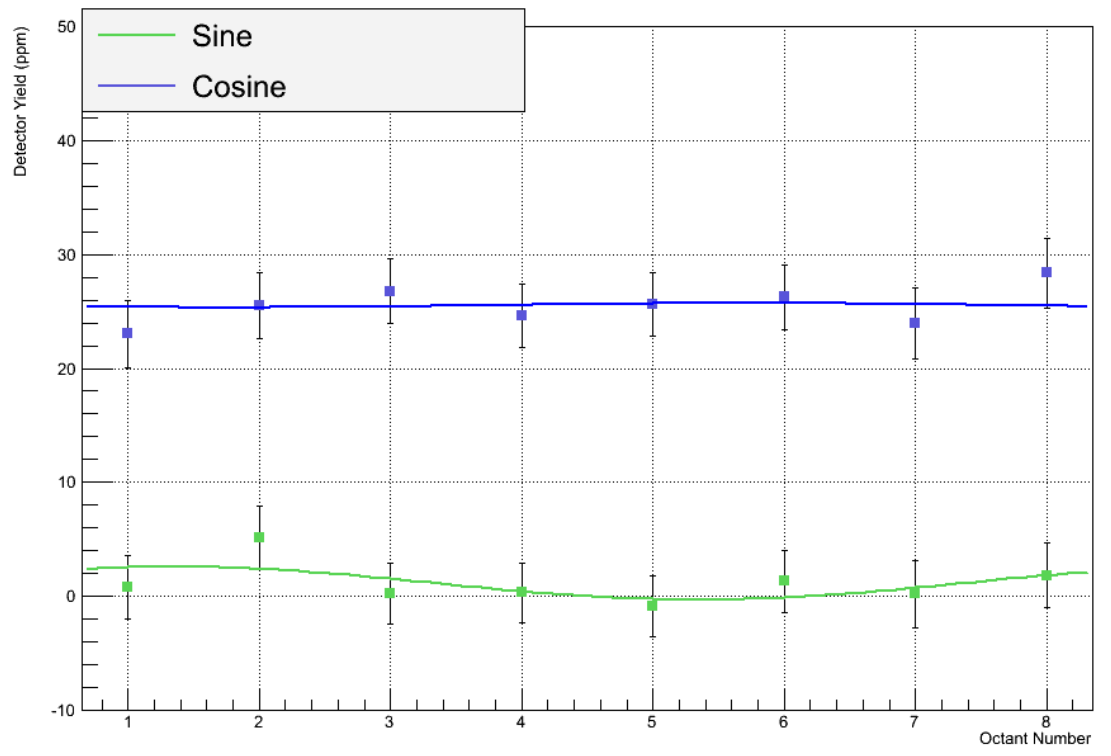

FIG. 4.40: Corrected yield response dipole to E modulation. 


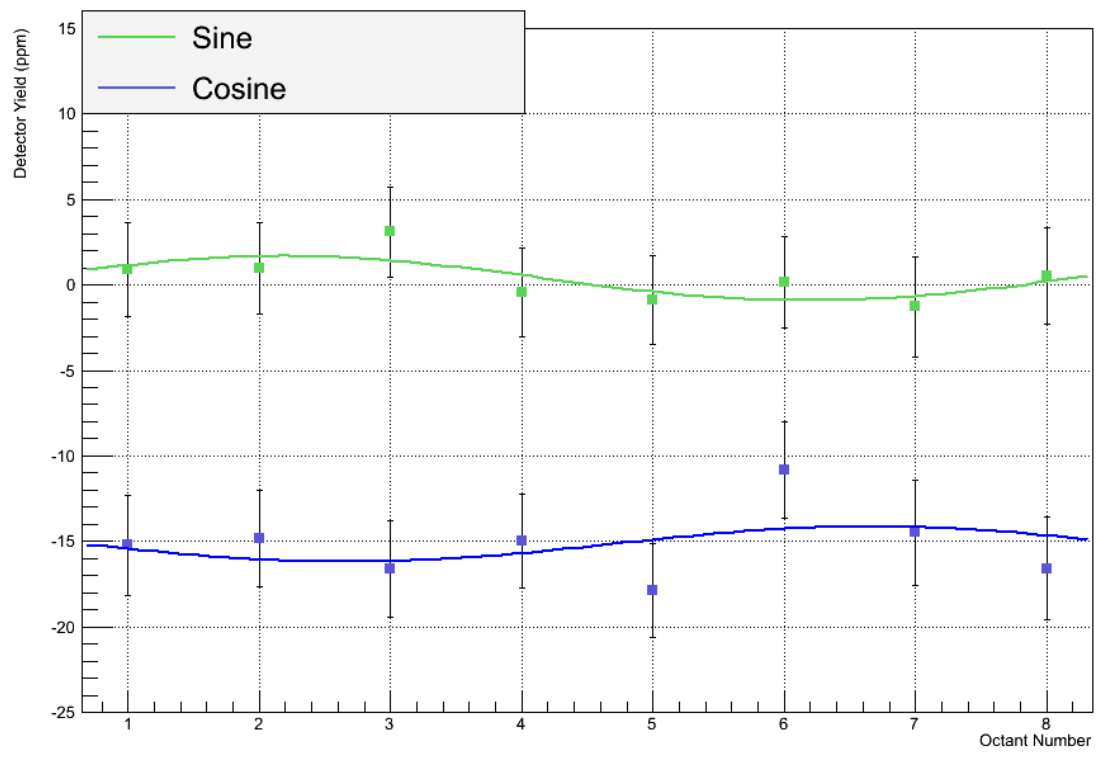

FIG. 4.41: Corrected yield response dipole to Y modulation.

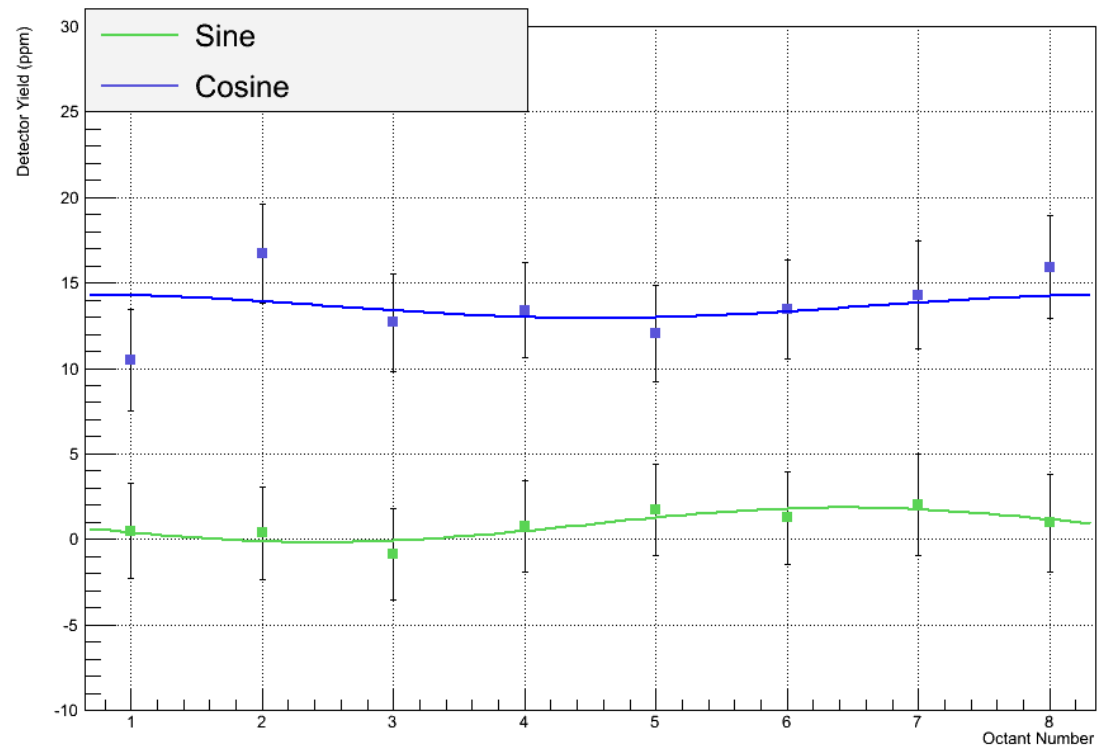

FIG. 4.42: Corrected yield response dipole to YP modulation.

All results are normalized by the average detector signal during a given run to 
make the results more interpretable. In some cases - particularly Y modulation the cosine component is shown to be comparable in size to the sine component. One obvious exception is during energy modulation when in fact there should be no cosine component. Recall that the energy feedback was disabled during energy modulation, therefore according to our model for the FFB interacting with the modulation, there should be no cosine component. There is, in-fact, a small cosine component that shows up in the fit, however it is small compared to the sine component $(\mathcal{O}(x 20)$ smaller). The corrected yield response for each modulation type can be seen in Fig. $4.38-4.42$.

A summary table of the results for both the corrected and uncorrected fits of the monopole and the dipole can be found in Tables 4.5 and 4.6. The detector dipole is a product of the fact that certain detectors are more sensitive to different modulation due to their spatial orientation; detectors 1 and 5 for instance, because they lie directly in the $\mathrm{X}$ plane, are more sensitive to X-modulation. By plotting the individual detector response as a function of octant, we expect to see a sinusoidal response with peaks at the detectors lying in the plane of modulation. The dipole describes the average detector response. The dipole response of the detectors is removed to $1 \sigma$ in all but one case; the one exception is the sine component of the $\mathrm{X}$ dipole where the result is only non-zero at the $1.9 \sigma$ level, i.e. not clearly significant. The essential flattening of the yield response using the Wien-average sensitivities is a good indicator that, at the fundamental level, the extraction of the sensitivities is being done correctly; the sensitivities are suppressed by more than 2 orders of magnitude. The change in the detector response monopole is less straight forward. The monopole represents the octant-independent response of the detectors in the same direction during the $\Delta \mathrm{X}$ caused by the modulation. The correction should remove the change regardless of the direction of the change resulting in a monopole 
of zero. Referring to Table 4.5, you can see that the monopole of the sine component - which is exceptionally large in the case of $\mathrm{E}$ for instance - is removed within errors. The cosine monopole, however, follows no discernible trend. Before correction, the monopole for each modulation type varies from small to very large. Once corrected, they show little change and in fact get larger in some cases. The general model to explain the monopole is that it is caused by changes in the backgrounds seen in the detectors, however the fact that only the sine component is corrected and the cosine seems random is not understood. The current model for this production of the monopole involves interaction of the beam halo with parts of the beamline, for instance the W-plug. Consider the beam halo as it passes through the W-plug, when the beam is modulated the halo, which is at a greater radius than the beam diameter, interacts with edges of the plug creating a background "glow" in the hall. Because the amount of interaction is tied directly to the modulation, the detectors see a beam position correlated background which affects all detectors equally.

\begin{tabular}{|l|r|r|r|r|}
\hline Mod Type & Sine (Raw) & Sine (Corrected) & Cosine (Raw) & Cosine (Corrected) \\
\hline $\mathrm{X}$ & $-31.7 \pm 0.9$ & $-1.7 \pm 1.0$ & $-108.9 \pm 1.0$ & $-111.4 \pm 1.0$ \\
\hline $\mathrm{X}^{\prime}$ & $1.0 \pm 0.9$ & $-0.2 \pm 1.0$ & $-60.6 \pm 1.0$ & $-22.1 \pm 1.0$ \\
\hline $\mathrm{E}(3 \mathrm{c} 12 \mathrm{X})$ & $577.6 \pm 0.9$ & $1.1 \pm 0.9$ & $-3.4 \pm 1.0$ & $25.5 \pm 1.0$ \\
\hline $\mathrm{Y}$ & $-10.7 \pm 0.9$ & $0.4 \pm 0.9$ & $-27.7 \pm 1.0$ & $-15.2 \pm 1.0$ \\
\hline $\mathrm{Y}^{\prime}$ & $19.6 \pm 0.9$ & $0.8 \pm 1.0$ & $12.1 \pm 1.0$ & $13.6 \pm 1.0$ \\
\hline
\end{tabular}

TABLE 4.5: Detector yield monopole in ppm before and after correction using Wien average modulation sensitivities.

False asymmetry present in the raw data causes the width of the asymmetry to become wider than the statistical width; correction of the raw asymmetry using beam modulation or linear regression should remove noise induced by the position sensitivities and reduce the asymmetry width. In the case of the linear regression, because the corrections are made to the data from which the sensitivities are extracted, 


\begin{tabular}{|c|r|r|r|r|}
\hline Mod Type & Sine (Raw) & Sine (Corrected) & Cosine (Raw) & Cosine (Corrected) \\
\hline $\mathrm{X}$ & $1248.9 \pm 1.3$ & $-2.7 \pm 1.4$ & $665.2 \pm 1.4$ & $-0.5 \pm 1.5$ \\
\hline $\mathrm{X}^{\prime}$ & $292.0 \pm 1.3$ & $1.1 \pm 1.4$ & $98.5 \pm 1.4$ & $-0.5 \pm 1.5$ \\
\hline $\mathrm{E}(3 \mathrm{c} 12 \mathrm{X})$ & $-412.9 \pm 1.3$ & $1.5 \pm 1.4$ & $20.4 \pm 1.4$ & $-0.2 \pm 1.4$ \\
\hline $\mathrm{Y}$ & $-452.8 \pm 1.3$ & $1.3 \pm 1.4$ & $-652.6 \pm 1.4$ & $-1.0 \pm 1.4$ \\
\hline $\mathrm{Y}^{\prime}$ & $984.2 \pm 1.3$ & $-1.0 \pm 1.4$ & $-110.9 \pm 1.4$ & $0.7 \pm 1.5$ \\
\hline
\end{tabular}

TABLE 4.6: Detector yield dipole response in ppm before and after correction using Wien average modulation sensitivities.

\begin{tabular}{|l|c|c|c|c|c|}
\hline DOF/Wien & $\mathrm{X}$ & $\mathrm{X}^{\prime}$ & $3 \mathrm{c} 12 \mathrm{X}$ & $\mathrm{Y}$ & $\mathrm{Y}^{\prime}$ \\
\hline Wien 1 & $-1687.6 \pm 18.5$ & $81.4 \pm 0.8$ & $-1462.3 \pm 2.1$ & $173.1 \pm 16.7$ & $0.3 \pm 0.4$ \\
\hline Wien 2 & $-1719.4 \pm 20.0$ & $81.3 \pm 0.8$ & $-1480.7 \pm 3.7$ & $214.5 \pm 17.3$ & $-1.1 \pm 0.6$ \\
\hline Wien 3 & $-1670.5 \pm 18.5$ & $80.6 \pm 0.9$ & $-1471.9 \pm 3.4$ & $232.9 \pm 18.5$ & $-2.3 \pm 1.2$ \\
\hline Wien 4 & $-1630.0 \pm 15.6$ & $82.2 \pm 0.7$ & $-1485.1 \pm 4.8$ & $295.2 \pm 7.8$ & $-3.9 \pm 0.8$ \\
\hline Wien 5 & $-1681.6 \pm 15.2$ & $82.8 \pm 0.8$ & $-1542.8 \pm 3.7$ & $274.3 \pm 12.3$ & $-2.1 \pm 0.6$ \\
\hline \hline Run I Avg. & $-1673.8 \pm 7.7$ & $81.8 \pm 0.4$ & $-1480.6 \pm 1.4$ & $264.1 \pm 5.5$ & $-1.1 \pm 0.3$ \\
\hline
\end{tabular}

TABLE 4.7: Wien-level sensitivities for the main detector system. The Run I value represents the error weighted average of each Wien-level result. Positions are given in $\mathrm{ppm} / \mathrm{mm}$ and angles are given in $\mathrm{ppm} / \mu \mathrm{rad}$.

it is mathematically required that the widths are reduced. This is not the case for the modulation-corrected data. While the modulation corrections are expected to reduce the raw width, it is not a mathematical requirement since the modulation extracted sensitivities are determined from an independent set of data. Below in Fig. 4.43 the main detector average corrected widths, for both beam modulation and linear regression Set 11 corrected asymmetries, are shown. The Set 11 linear regression set uses the same independent variables as the beam modulation. The beam modulation corrections were done using Wien-averaged sensitivities and the LRB corrections were done using both quartet and the Wien-average sensitivities.

Both the linear regression and the modulation corrections are shown to reduce the asymmetry width. The greatest width reduction comes from the quartet-level linear regression corrections, but all methods had a positive effect on the asymmetry 


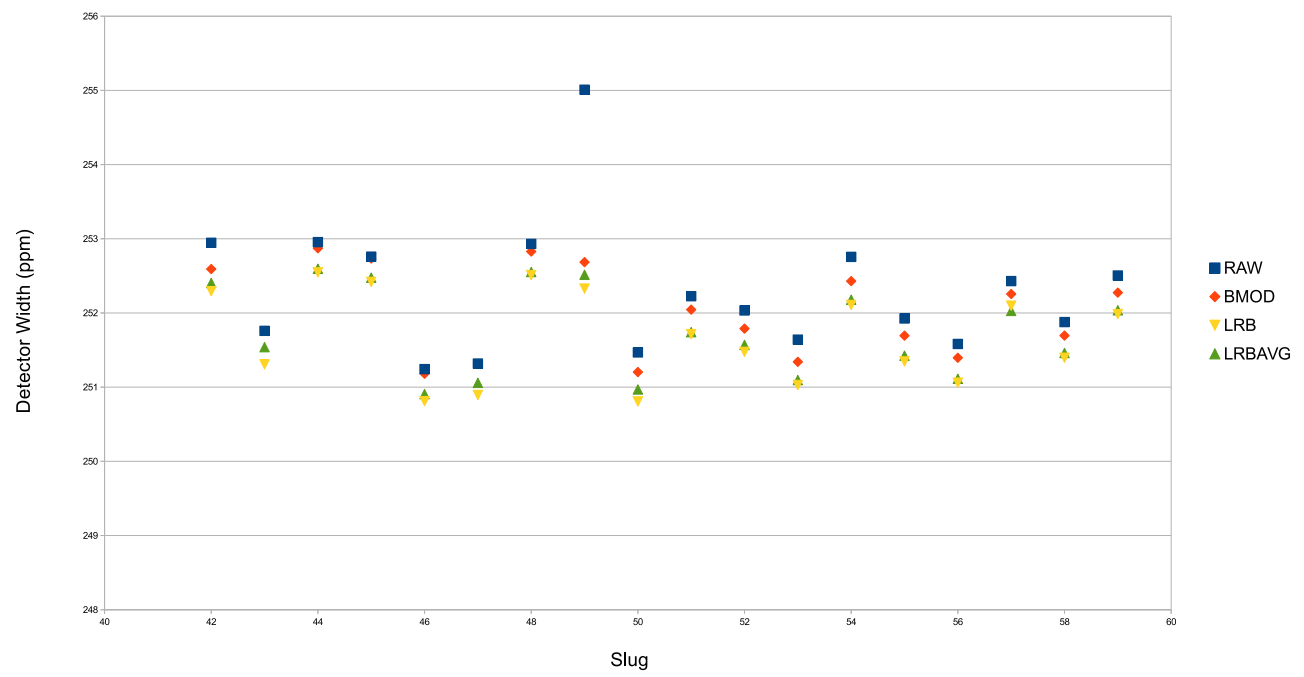

FIG. 4.43: The slug average detector widths are shown for raw, corrected via modulation, LRB, and wien average LRB for slugs 42- 59. The maximum reduction in width is achieved using the quartet level LRB sensitivities.

width. The fact that the width reduction due to the Wien-average LRB sensitivities is worse than the quartet level corrected widths is suggestive of a hidden variable. An argument could be made that the sensitivities are changing with time and therefore the Wien average corrections are wrong, however this seems unlikely given the stability of the beam-modulation extracted sensitivities. As a note, changing values of the slug average widths are expected due to changing beam properties during each slug; a run period such as slug 49 could simply be due to a section of time with unusually large position differences. The average beam current during Run 1 was $156 \mu \mathrm{A}$ and the main detector current weighted rate was $\sim 4.4 \mathrm{MHz} / \mu \mathrm{A}[58]$. This implies that the combined detector width due to counting statistics alone should be $\mathcal{O}(209)$ ppm per $4 \mathrm{~ms}$ quartet. The remaining noise is likely due to other sources of noise such as beamline backgrounds, detector energy resolution, BCM resolution, and target boiling, among others. 


\begin{tabular}{|l|c|c|c|c|c|}
\hline DOF/Wien & $\mathrm{X}$ & $\mathrm{X}^{\prime}\left(\mathrm{x} 10^{-2}\right)$ & $3 \mathrm{c} 12 \mathrm{X}$ & $\mathrm{Y}$ & $\mathrm{Y}^{\prime}\left(\mathrm{x} 10^{-2}\right)$ \\
\hline Wien 1 & $1.1 \pm 1.9$ & $-18.4 \pm 4.2$ & $1.4 \pm 1.2$ & $18.7 \pm 1.5$ & $81.3 \pm 5.1$ \\
\hline Wien 2 & $11.1 \pm 1.1$ & $-14.5 \pm 3.3$ & $-29.9 \pm 1.0$ & $-11.7 \pm 1.1$ & $41.7 \pm 3.7$ \\
\hline Wien 3 & $-20.6 \pm 1.5$ & $-70.3 \pm 4.1$ & $-6.8 \pm 0.9$ & $-7.3 \pm 1.5$ & $-37.2 \pm 4.5$ \\
\hline Wien 4 & $2.0 \pm 1.4$ & $7.8 \pm 4.1$ & $8.4 \pm 0.9$ & $-20.9 \pm 1.1$ & $-40.6 \pm 3.8$ \\
\hline Wien 5 & $3.5 \pm 1.5$ & $13.6 \pm 4.5$ & $14.2 \pm 0.9$ & $-6.4 \pm 1.2$ & $-36.4 \pm 4.2$ \\
\hline
\end{tabular}

TABLE 4.8: Wien-Average Position Difference for Run I after $6 \sigma$ cut. Positions are given in $\mathrm{nm}$ and angles are given in nrad.

\subsubsection{Residual Correlations}

As discussed above it is important to understand how well the corrections that are being applied to the data are removing the detector sensitivities. Study of the yield corrections shows that, while to first order the corrections are doing what they are intended, i.e. correcting the sinusoidal component of the detector response to beam motion, there are still unexplained effects such as monopoles left in the detector response. Effects such as charge non-linearities, beam halo, and beamline background effects can contribute to the detector signal as hidden variables. It is important to understand the degree to which these and other effects contribute to the corrected asymmetry in the form of residual correlations. This was studied by looking at the correlation of the corrected asymmetry to each independent variable on the Wien level over Run I. Studying this on the Wien level was done to take advantage of cancellations resulting from the IHWP flips and averaging out of statistical noise. Table 4.9 shows the results of the study for each Wien in terms of the residual correlation of corrected main detector asymmetry to each DOF. The results show that, at least on the Wien level, the residual correlations are $\lesssim 2 \sigma$. Due to this no additional correction was applied to the final asymmetry. 


\begin{tabular}{|l|r|r|r|r|r|}
\hline DOF & Wien 1 & Wien 2 & Wien 3 & Wien 4 & Wien 5 \\
\hline $\mathrm{X}$ & $0.7 \pm 0.6$ & $0.3 \pm 0.6$ & $0.3 \pm 0.6$ & $-0.3 \pm 0.6$ & $-0.4 \pm 0.7$ \\
\hline $\mathrm{X}^{\prime}$ & $23.2 \pm 26.2$ & $1.0 \pm 22.0$ & $7.0 \pm 21.1$ & $-3.6 \pm 19.6$ & $-8.8 \pm 25.0$ \\
\hline $\mathrm{E}$ & $-1.0 \pm 0.9$ & $-0.3 \pm 0.7$ & $-0.1 \pm 0.9$ & $0.4 \pm 0.6$ & $-0.2 \pm 1.1$ \\
\hline $\mathrm{Y}$ & $0.2 \pm 0.8$ & $1.0 \pm 0.7$ & $-0.2 \pm 0.6$ & $-0.1 \pm 0.8$ & $0.2 \pm 0.9$ \\
\hline $\mathrm{Y}^{\prime}$ & $20.0 \pm 22.4$ & $33.6 \pm 19.9$ & $-5.6 \pm 19.3$ & $-12.9 \pm 22.5$ & $14.9 \pm 24.8$ \\
\hline
\end{tabular}

TABLE 4.9: The residual correlations of the main detector to each degree of freedom in the beam after beam modulation corrections. Each corrections was determined and is listed as the Wien average value. Position correlations are given in $\mathrm{ppb} / \mathrm{nm}$ and angular correlations are given in $\mathrm{ppb} / \mathrm{nrad}$.

\subsubsection{Average Position Differences}

The Run I Wien-Average helicity-correlated position differences for each of the beam parameters are given in Table 4.8. Each beam parameter is weighted by the main detector error using the prescription found in Appendix A.1. Calculation of the Wien-average position differences was subject to a data quality cut given by

$$
\left|x_{i}-\langle x\rangle\right|<n_{\sigma} \sqrt{\sigma_{x_{i}}^{2}+\sigma_{\langle x\rangle}^{2}}
$$

where $x_{i}$ is the position difference for each runlet, $\langle x\rangle$ is the detector-weighted Wienaverage position difference before cuts, $\sigma_{x_{i}}^{2}$ is the runlet error, and $\sigma_{\langle x\rangle}^{2}$ is the error on the Wien average position difference. The size of the cut was defined by $n_{\sigma}$, which was the number of sigma from the mean that was acceptable. For the purpose of this analysis, $n_{\sigma}$ was set to be six; a six sigma cut removed the major outliers in the data while leaving most of the data intact. The position differences are also subject to a number of beam stability cuts inside the standard analyser as well as cuts on whether there were failures in any of the linear regression schemes. Shown in Table 4.10 are the number of events lost for different cuts. The number of events lost due to more stringent cuts is relatively small up to $6 \sigma$. 


\begin{tabular}{|r|c|r|c|}
\hline Runlet Cut $(\sigma)$ & Runlet Count & Runlets Lost & \% Lost \\
\hline 100 & 5674 & 0 & 0.0 \\
\hline 10 & 5650 & 24 & 0.4 \\
\hline 9 & 5633 & 41 & 0.7 \\
\hline 8 & 5574 & 100 & 1.7 \\
\hline 7 & 5478 & 196 & 3.4 \\
\hline 6 & 5291 & 383 & 6.7 \\
\hline
\end{tabular}

TABLE 4.10: The number of runlets lost due to $\mathrm{n} \sigma$ cuts applied to the position difference data.

\subsubsection{Asymmetry Correction}

Using the extracted Slug-average sensitivities found in Table 4.7 and the detectorweighted position differences found in Table 4.8, the correction to the measured asymmetry due to each beam parameter was computed and is displayed in Table 4.11 .

\begin{tabular}{|l|c|c|c|c|c|}
\hline DOF/Wien & $\mathrm{X}(\mathrm{ppb})$ & $\mathrm{X}^{\prime}(\mathrm{ppb})$ & 3c12X $(\mathrm{ppb})$ & $\mathrm{Y}(\mathrm{ppb})$ & $\mathrm{Y}^{\prime}(\mathrm{ppb})$ \\
\hline Wien 1 & $-1.9 \pm 3.2$ & $-15.0 \pm 3.4$ & $-2.0 \pm 1.8$ & $3.2 \pm 0.4$ & $0.2 \pm 0.4$ \\
\hline Wien 2 & $-19.0 \pm 1.9$ & $-11.8 \pm 2.7$ & $44.4 \pm 1.5$ & $-2.5 \pm 0.3$ & $-0.5 \pm 0.2$ \\
\hline Wien 3 & $34.4 \pm 2.5$ & $-56.6 \pm 3.3$ & $10.1 \pm 1.3$ & $-1.7 \pm 0.4$ & $0.8 \pm 0.5$ \\
\hline Wien 4 & $-3.2 \pm 2.3$ & $6.4 \pm 3.3$ & $-12.5 \pm 1.4$ & $-6.2 \pm 0.4$ & $1.6 \pm 0.4$ \\
\hline Wien 5 & $-5.8 \pm 2.6$ & $11.3 \pm 3.7$ & $-21.9 \pm 1.4$ & $-1.8 \pm 0.3$ & $0.8 \pm 0.3$ \\
\hline \hline Total (ppb) & Wien 1 & Wien 2 & Wien 3 & Wien 4 & Wien 5 \\
\hline Correction & $-15.5 \pm 5.0$ & $10.6 \pm 3.6$ & $-13.0 \pm 4.4$ & $-13.9 \pm 4.3$ & $-17.5 \pm 4.8$ \\
\hline
\end{tabular}

TABLE 4.11: Run I modulation corrections to the measured asymmetries by Wien.

The raw asymmetry - this asymmetry was subject to the data quality cut mentioned above in 4.1.6 - was corrected using the Slug-average beam modulation sensitivities. A plot of the corrected asymmetry for both IN and OUT IHWP states is shown in Fig. 4.44. Each point shown is the slug average, sign corrected, modulation corrected asymmetry. An important factor in determining the quality of the modulations corrections is whether the null asymmetry is consistent with zero or not. Changes in the Wien and IHWP states merely flip the sign of the asymmetry 
in the data, because different states should measure the same physics, looking at the difference between the IN and OUT state data is a useful way to gain insight into the quality of the correction being applied and find hints of potential hidden variables. After modulation correction the null asymmetry was found to be -25.51 $\pm 13.54 \mathrm{ppb}$ which is not significantly non-zero. The slug-average asymmetry values are consistent with-in errors and give an error-weighted average asymmetry for Run I of $-232.8 \pm 13.54 \mathrm{ppb}$.

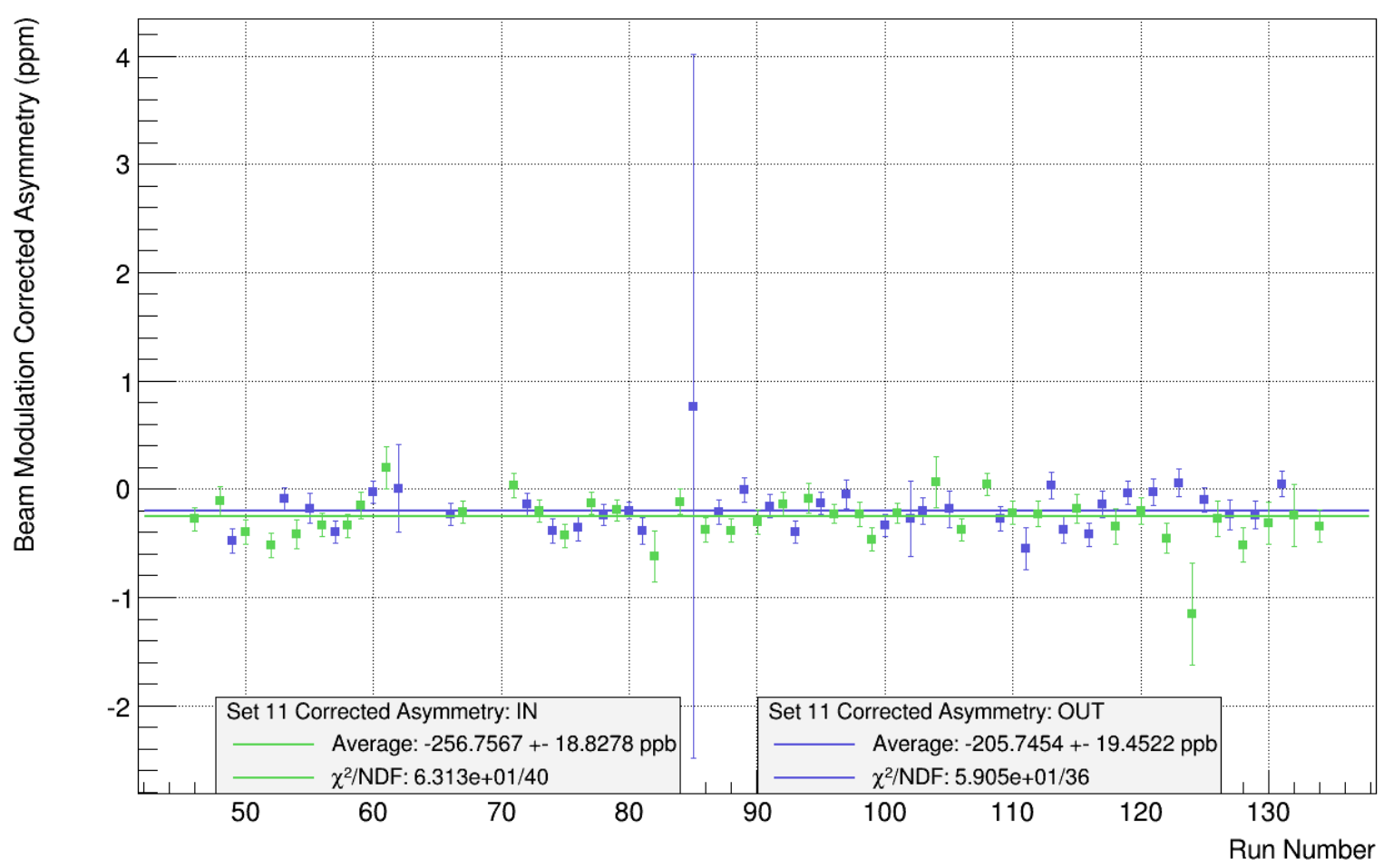

FIG. 4.44: The beam-modulation corrected asymmetry for both IN and OUT IHWP settings. 


\section{CHAPTER 5}

\section{Systematic Uncertainties}

\section{1 $\mathrm{Q}^{2}$ Systematics}

\subsubsection{Scattering Kinematics}

Precise determination of the proton's weak-charge, $\mathrm{Q}_{w}^{p}$, to $4 \%$ requires knowledge of the four-momentum transfer, $\mathrm{Q}^{2}$, to an uncertainty of $0.5 \%$. For the purpose of this analysis this level of precision is not required and the $\mathrm{Q}^{2}$ determination will be based solely on the data set defined as Wien 0 .

The elastic asymmetry for $\mathrm{Q}_{w}^{p}$ kinematics is a function of $\mathrm{Q}^{2}$, thus making precise knowledge of $\mathrm{Q}^{2}$ a prerequisite to a precise measure of $\mathrm{Q}_{w}^{p}$; recall that

$$
A=\frac{G_{F}}{4 \pi \alpha \sqrt{2}}\left[Q_{w}^{p} Q^{2}+B\left(Q^{2}\right) Q^{4}\right],
$$

where $\mathrm{G}_{F}$ is the Fermi constant, $\alpha$ is the fine-structure constant, and $\mathrm{B}\left(\mathrm{Q}^{2}\right)$ contains contributions from the hadronic form factors. In the case of relativistic electrons scattering elastically from the protons in the liquid Hydrogen target, the momentum 
transfer can be written as,

$$
Q^{2}=4 E E^{\prime} \sin ^{2} \frac{\theta}{2}
$$

Here, $\mathrm{E}$ is the incident energy of the incoming electron, $\mathrm{E}^{\prime}$ is the energy of the scattered electron, and $\theta$ is the scattering angle of the outgoing electron. Because the $\mathrm{Q}_{\text {weak }}$ kinematics are two-body elastic scattering, only two of the three variables are required to determine $\mathrm{Q}^{2}$. With this in mind, some thought must go into choosing the set of variables that will give us the most precise measurement. The incident electron energy is determined by measuring the beam energy, corrected for initialstate losses in the target, as discussed in 5.1.2, and is very well determined. The scattering angle, $\theta$, is determined using Region II data in conjunction with survey data defining the geometry of the experiment. The post-scattering energy, $\mathrm{E}^{\prime}$, is the least well-known of the kinematic variables. Determination of $\mathrm{E}^{\prime}$ requires position and angle information from both Region II and Region III, as well as precise mapping of the spectrometer B-field. Given that the incident energy and scattering angle are directly measured, they present the best choice; $\mathrm{E}^{\prime}$ will be used as a verification of our measurement as well as a check that the events we see are truly elastic events. Using only the kinematic variables of interest, $\mathrm{Q}^{2}$ can be rewritten independent of $\mathrm{E}^{\prime}$ as

$$
Q^{2}=2 E^{2} \frac{(1-\cos \theta)}{1+\frac{E}{M_{p}}(1-\cos \theta)} .
$$

One complication is that while we can measure both the incident beam energy and the scattering angle, we are interested specifically in the values at the scattering vertex. Determination of $\mathrm{E}$ and $\theta$ at the scattering vertex is complicated due to external radiative effects, internal bremsstrahlung, multiple scattering, and energy loss $(d E / d x)$ in the target. The solution is to use simulation to handle the internal and external processes listed above, and experimental results for the measured inci- 
dent beam energy entering the target and scattered angle exiting the target, as well as other relevant quantities such as calculation of the $\mathrm{Z}_{\text {vertex }}$ and $\mathrm{E}^{\prime}$, to benchmark the simulation. Tuning the simulation until the simulated values match the experimentally measured observables allows for the extraction of $\mathrm{E}$ and $\theta$ at the scattering vertex.

\subsubsection{Beam Energy}

The precision measurement of the beam energy in Hall C uses a set of superharps at the entrance and exit of the Hall $\mathrm{C}$ arc. The superharps provide absolute beam position determination using a $10 \mu \mathrm{m}$ wire which is passed through the beam using a shaft position encoder [71]. When the superharp wire passes through the beam a signal is produced in the read-out electronics. The mean of the peak of the output voltage distribution, when referenced to the position read-out of the encoder, provides the position and width of the beam. The resolution of the beam position determination is limited by the width of the superharp wire.

The absolute beam energy measurement is completed by powering only the dipole magnets in the arc; all quadrupoles, sextupoles, and correctors are turned off. The current in the dipole magnets is calibrated to center the electron beam through the dipoles. Using the position measurements at the entrance and exit of the arc, along with the results from accurate mapping of the dipole fields, the momentum of the electron beam is computed using

$$
E=\frac{e}{\Theta} \int B d l
$$

Here $\int B d l$ is the magnetic field integral along the beam path through the arc and $\Theta$ is the bending angle. Using this method, absolute beam energy measure- 
ments $\mathcal{O}\left(10^{-3}\right)$ are possible [72]. Once the absolute energy scale has been set, the quadrupoles and sextupoles are energized and correctors are used to re-center the beam. Using a harp in the center of the arc to determine the variation in beam position allows the relative beam energy to be determined to $\mathcal{O}\left(10^{-4}\right)$.

\subsubsection{Scattering Angle}

The visible scattering angle, which describes the angular deviation from the Z-axis along the beam-line, is measured primarily using the HDCs as described in 3.6.1. The HDCs, which are placed in pairs diametrically opposed to each other in front of QTOR, have an angular scattering acceptance of $4^{\circ}-12^{\circ}$. The signal in the HDCs was dominated by Møller electrons and operated at a beam current of $100 \mathrm{pA}$; the VDC signal at this current was completely dominated by cosmic rays. In order to select good tracks both the HDC and VDCs were needed; good tracks were identified as tracks with an $\mathrm{E}^{\prime}$ that is consistent with an elastic electron, ie. a track can be formed between Region II and Region III through QTOR which hits the trigger scintillator.

\section{$5.1 .4 \mathrm{Q}^{2}$}

As discussed in Sec. 5.1.1 the determination of the acceptance-averaged effective momentum transfer, $\left\langle Q^{2}\right\rangle_{\text {tree }}$, is computed according to the method found in [73], and depends on the incident beam energy at the scattering vertex and the effective scattering angle (see Eq. 5.3). The final $\left\langle Q^{2}\right\rangle_{\text {tree }}$ is computed using GEANT4 and is benchmarked using the tracking data. This is an important step because the experiment cannot directly measure the scattering energy and angle at the vertex. The incident beam energy is well known, however what is needed for determination 
of $\left\langle Q^{2}\right\rangle_{\text {tree }}$ is the incident energy at the scattering vertex. This is computed using GEANT4 to account for energy losses in the target. The energy at the vertex is then computed using

$$
E_{\text {vertex }}=\left\langle E_{\text {beam }}-\frac{d E_{\text {beam }}}{d Z} \Delta Z\right\rangle
$$

Here, $E_{\text {beam }}$ is the incident beam energy on the target and $\Delta Z$ is the distance travelled through the target before scattering. The Run I beam energy was computed to be

$$
E_{\text {vertex }}=1155 \pm 3 \mathrm{MeV} \text {. }
$$

At the time of writing the analysis of the Run I acceptance averaged momentum transfer was still in-progress. For this reason, the Wien 0 value was used. The Wien 0 tree level momentum transfer was found to be [36]

$$
\left\langle Q^{2}\right\rangle_{\text {tree }}^{\text {sim }}=0.0250 \pm 0.0006(\mathrm{GeV} / \mathrm{c})^{2} .
$$

The effective scattering angle can be computed using the vertex values for the momentum transfer and incident beam energy, calculated above, along with Eq. 5.3.

$$
\left\langle\theta_{\text {eff }}\right\rangle=7.90 \pm 0.30^{\circ}
$$

\section{$5.2 \quad$ Backgrounds}

Precise extraction of the physics asymmetry requires understanding of the backgrounds which dilute the detector signal and give false asymmetries. The signal

dilution due to backgrounds is the fractional contribution of the total signal that 
comes from background processes and is defined by

$$
f_{\mathrm{bg}}=\frac{Y_{\mathrm{bg}}}{Y_{\mathrm{bg}}+Y_{\text {signal }}}
$$

The total contribution to the measured asymmetry can be written as

$$
A_{\text {measured }}=P\left[\left(1-\Sigma_{i} f_{\mathrm{bg}}^{i}\right) A_{\mathrm{physics}}+\Sigma_{i} f_{\mathrm{bg}}^{i} A_{\mathrm{bg}}^{i}\right]
$$

In order to remove the background contributions, both the signal dilution and the background asymmetry must be measured or estimated. Background contributions from the Aluminium target windows, soft backgrounds, inelastic background contributions, and neutral backgrounds are discussed in more detail below.

\subsubsection{Aluminium Target Window Background}

Electrons scattering from the primary target interact, not only with the $\mathrm{H}_{2}$ in the target, but also with the upstream (US) and downstream (DS) target windows. The effects on the measured asymmetry due to scattering from the target windows comes in two parts: the asymmetry of electron-aluminium scattering and a dilution of the measured signal in the detectors. Both of these effects were extracted using stand-alone measurements which are explained in more detail below.

\subsubsection{Aluminium Target Asymmetry}

In the same way as electron scattering from hydrogen, scattering from the target windows produces a measurable parity-violating asymmetry in the detectors. The scattering from the target windows leads to $\mathrm{a} \simeq 30 \%$ correction to the physics asymmetry during extraction from the measured value and is the largest correction 
made to the weak-charge. The electron-aluminium asymmetry is approximated in Eq. 5.11 in terms of the weak-charge of the proton and neutron.

$$
A_{P V}=\frac{-G_{F} Q^{2}}{4 \pi \alpha \sqrt{2}}\left[Q_{W}^{p}+Q_{W}^{n}\left(\frac{N}{Z}\right)\right]
$$

The size of the asymmetry is driven by the fact that the weak-charge of the neutron is an order of magnitude larger than that of the proton. In order to measure the aluminium asymmetry, the target apparatus, which could be moved using linear stepper motors, included an array of aluminium targets of varying radiation lengths machined from the same material as the target windows; in the case of the upstream (US) targets 1\%, 2\%, $4 \%$ radiation lengths and the downstream (DS) targets $2 \%, 4 \%$, and $8 \%$ radiation lengths. The primary measurement of the aluminium asymmetry was done using the $4 \%$ DS target. One subtlety to consider is that on the $\mathrm{lH}_{2}$ target the US and DS windows are at different locations in $\mathrm{Z}$ along the beamline. Because of this, the scattering profile on the detectors from each window is different, in-fact part of the scattering profile of the US window falls off the detector bars. This leads to a different asymmetry from the US and DS windows; the asymmetry is different because of the difference in the kinematic acceptance between the US and DS windows. To account for this difference, a small, simulation extracted, correction is applied to the asymmetry measured on the $4 \%$ DS target. The regressed aluminium asymmetry was computed in [74] and is shown for the standard linear regression set in Fig.5.1. The aluminium asymmetry must also be corrected for the polarization and background contributions[75]. With the addition of radiative corrections [76] the final Wien 0 aluminium asymmetry is found to be

$$
A_{\mathrm{b} 1}=1.76 \pm 0.18 \text { (stat.) } \pm 0.18 \text { (syst.) } \pm 0.04 \text { (model) ppm. }
$$


The Wien 0 result is used in this analysis due to the unavailability of the Run 1 results.

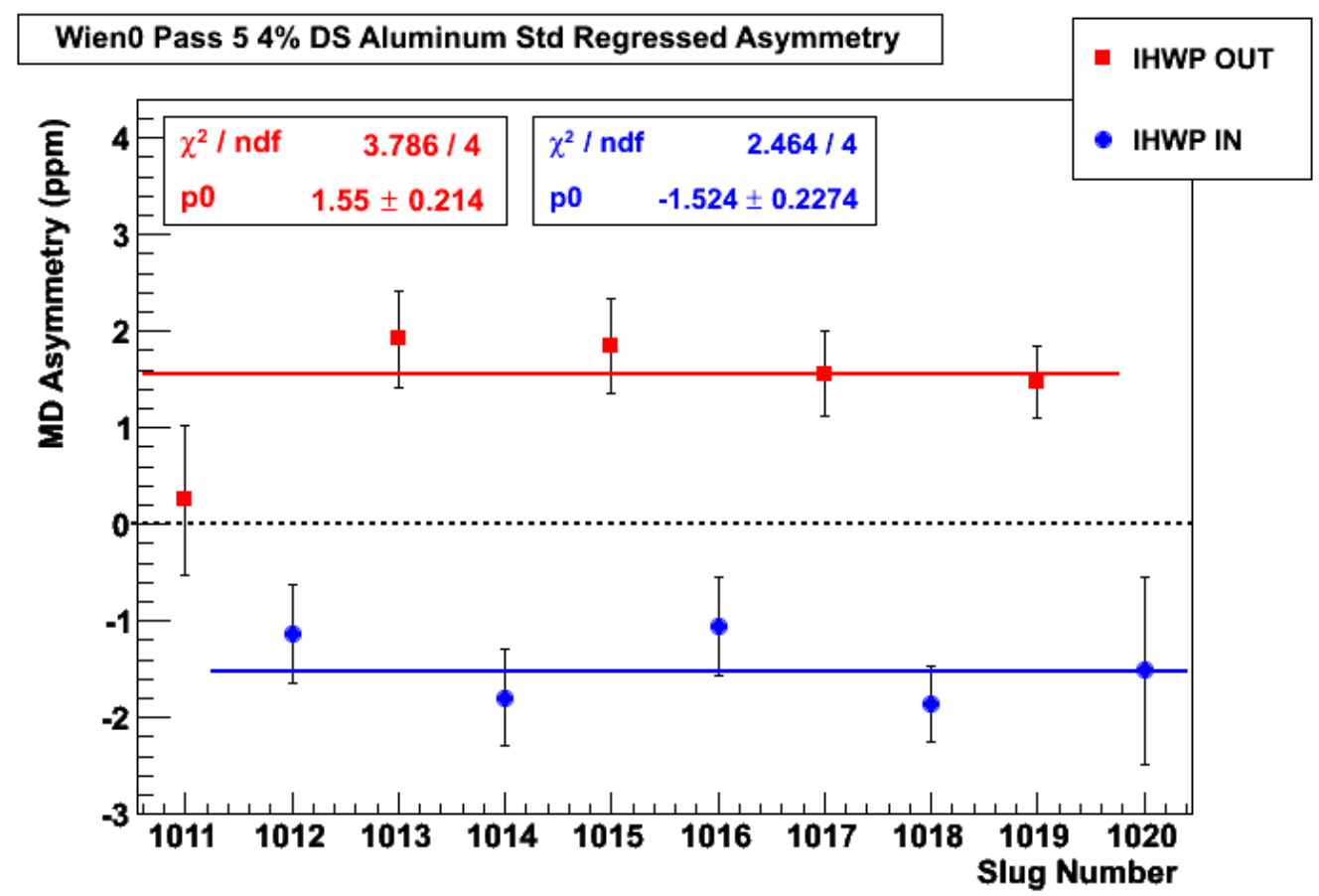

FIG. 5.1: Regressed aluminium asymmetry plotted versus slug for both IN and OUT half-wave plate settings. This does not include corrections due to polarization or backgrounds.

\subsubsection{Aluminium Dilution}

The dilution of the measured yield due to electron scattering from the aluminium windows was measured using two techniques: evacuated cell method and gas extraction method. The easiest of the two methods is the former in which the target cell is evacuated and the scattering signal is recorded. The cell is then refilled and another measurement is taken. In the full condition, the liquid hydrogen works to cool the target windows. Because the liquid hydrogen is needed for cooling, one concern with the evacuated method was potential thermal and structural damage 
to the windows. Because of this the measurements were done at much lower currents: $0.2-1.0 \mu \mathrm{A}$. One thing to note is that there were several hours between the evacuated and full measurements due, in part, to the time required to refill and cool the target. In order to eliminate potential errors in the measurement due to time dependent changes, and BCM calibration uncertainties, a reference target, the yield from which should not change over time, was used to normalize both measurements:

$$
R_{\text {empty }}^{\text {norm }}=\frac{R_{\text {empty }}}{R_{\text {reference }}}, \quad R_{\text {full }}^{\text {norm }}=\frac{R_{\text {full }}}{R_{\text {reference }}}
$$

A $0.5 \%$ radiation length carbon target, located on the DS target ladder, was used as a reference. The dilution factor is the ratio of $R_{\text {empty }}^{\text {norm }} / R_{\text {full }}^{\text {norm }}$. As before, contributions from the US and DS windows must be treated separately. The process of applying the radiative corrections is guided by studies of aluminium targets of different thickness and simulations. The Run I dilution factor can be seen in Fig.5.2 for opposing octants. A discrepancy of $\sim 2.4 \%$ can be seen between the normalized and unnormalized measurements which is attributed to the difference in reference target yields [77]. The final extracted dilution, including corrections [78], is given by

$$
f_{\mathrm{b} 1}=0.03226 \pm 0.0001 \text { (stat.) } \pm 0.0004 \text { (syst.) } \pm 0.0021 \text { (model) }
$$

The gas extraction method uses gaseous hydrogen in the target at different pressures to determine the signal in the detectors versus pressure. By extrapolating to zero pressure, the empty target signal can be determined, allowing for the extraction of the dilution factor. While this method of measurement serves as a check on the evacuated method, it is not the cleanest method of extraction. Uncertainties such 


\section{Raw Aluminum Dilution Factor (run 1)}

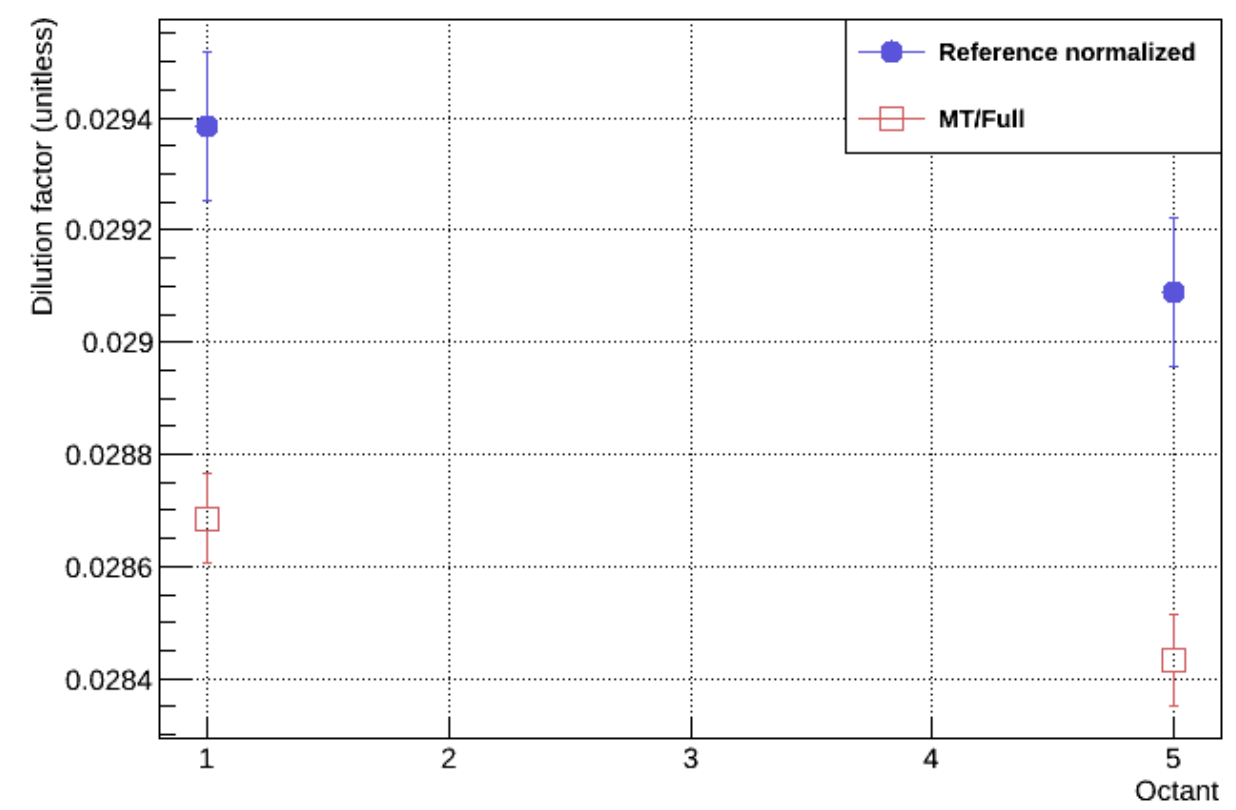

FIG. 5.2: The Run I Dilution for opposing octants is shown for both normalized and unnormalized cases. The discrepancy between with and without normalisation is attributed to a difference in the reference target yields.

as local density changes due to heating, electronic deadtime, radiative corrections due to the addition of hydrogen to the target cell, and BCM linearity between the gas and full states make this not the optimal approach.

\subsubsection{Beamline Background}

The beamline background encompasses low energy events hitting the main detectors not originating from elastic scattering in the target. Possible sources include: showers from the collimator and shield wall, octant-to-octant cross-talk, and beamline scattering. These background sources, while somewhat smaller than the Aluminium target window background, are an important contribution to the measured asymmetry. In general, much of this is suppressed via shielding and the lead pre-radiators installed directly in front of the main detectors and the tungsten plug 
which was installed in the downstream side of the first collimator. The pre-radiators are effective at suppressing low energy events entering the detectors while also increasing the size of the elastic events from the target.

\subsubsection{Beamline Background Dilution}

Measurement of the beamline background dilution was done using a blocked octant technique. In order to eliminate line-of-site from the target events, 3" of lead was installed directly downstream of the defining collimator in octant 7. Blocking the octant allowed for a measurement of background dilution as the ratio of the blocked signal to the unblocked total signal. Blocked octant measurements were taken in May 2011 at two QTOR currents, 8921 A and 6700 A (inelastic), and on both the $l \mathrm{H}_{2}$ and $4 \%$ DS aluminium targets. A more detailed discussion of the blocked octant study can be found in K. Meyers thesis [8] and Wade Duvall's analysis summary[79]. The results of the blocked octant study give an upper bound on the beamline dilution of

$$
f_{\mathrm{b} 3}=0.00218 \pm 0.00064 \text {. }
$$

The average of the blocked octant data, including good and bad halo periods, was used in previous analyses. Due to the increased correlation between the main detectors and the background monitors described below it was decided that a conservative estimate on the Run I dilution was more appropriate. The value used is based on values extracted from bad halo data. This is expected to be an upper bound due to the fact that significant increases would mean significantly more beam being dumped into the tungsten plug, accompanied by increased temperatures in the plug readings, and frequent beam trips[80]. This is not something that was observed. 


\section{Beamline Background Asymmetry}

The asymmetry contribution due to beamline background sources was determined using results from the blocked octant study [79], however it was not done directly. The blocked octant study was completed near the end of Run II data taking and there was no analogue in Run I. Because of this and the fact that the MD9 background detector was moved during Run II the USLUMIs are used as a common element between the runs to indirectly measure the beamline background outside of the blocked octant study. The details of how this is done can be found in Mark Pitt's log entry [81]. According to the blocked octant study analysis the following relationships can be derived.

$$
\begin{aligned}
& A_{\text {unblocked }}^{\mathrm{MD}}=0.0018 \times A_{\text {blocked }}^{\mathrm{MD}} \\
& A_{\text {unblocked }}^{\mathrm{MD} 9}=0.094 \times A_{\text {blocked }}^{\mathrm{MD} 9} \\
& A_{\text {unblocked }}^{\mathrm{MD}}=\left(\frac{f_{\mathrm{MD}}}{f_{\mathrm{MD} 9}}\right)\left(\frac{A_{\text {blocked }}^{\mathrm{MD}}}{A_{\text {blocked }}^{\mathrm{MD}}}\right) A_{\text {unblocked }}^{\mathrm{MD} 9} \\
& A_{\mathrm{unblocked}}^{\mathrm{MD}}=0.044 \times A_{\text {unblocked }}^{\mathrm{MD} 9}
\end{aligned}
$$

Here the coefficient in Eq.5.16b and 5.16c gives the measured dilution factor and Eq.5.16d gives the relation between the unblocked detector signals in terms of the dilution factors and the measured blocked octant values. Physically, Eq.5.16d gives the ratio of the background asymmetry contributions in terms of MD and MD9. This is tied to the USLUMI by looking at the ratio of the asymmetries between MD9 and the USLUMI during Run I.

$$
A_{\text {unblocked }}^{\mathrm{MD} 9}=0.626 \times A_{\text {unblocked }}^{\text {USLUMI }}
$$


Putting Eq. 5.16d and 5.17 together we get

$$
A_{\text {unblocked }}^{\mathrm{MD}}=(0.0276 \pm 0.0276) \times A_{\text {unblocked }}^{\text {USLUMI }} .
$$

An error bar of $100 \%$ has been assigned due to uncertainties in the fraction of the signal seen in MD9 that is from beamline background. Using this relationship the average beamline asymmetry was determined to be

$$
A_{\mathrm{b} 3}=10.94 \pm 10.95 \mathrm{ppb}
$$

\subsubsection{Inelastic Background}

The inelastic background is from inelastic scattering events, dominantly from the nuclear $N \rightarrow \Delta$ transition, which fall into the experimental acceptance of the main detectors. The inelastic asymmetry was expected to be $\mathcal{O}(10)$ larger that than the elastic asymmetry, however the fraction of inelastic events in the detectors to that of the elastic events makes the total contribution to the total asymmetry

relatively small. Measurement of the inelastic asymmetry was done by changing the QTOR current to a value that maximized the inelastic signal in the detectors; according to simulation (Fig. 5.3) this was 6700 A. By changing the QTOR current from 8921 A to 6700 A the fractional contribution of inelastic to elastic events was increased $\mathcal{O}(100)$ [13]. The inelastic asymmetry was found to be [13]

$$
A_{\mathrm{b} 4}=3.02 \pm 0.97 \mathrm{ppm}
$$




\section{F inel vs QTOR current}

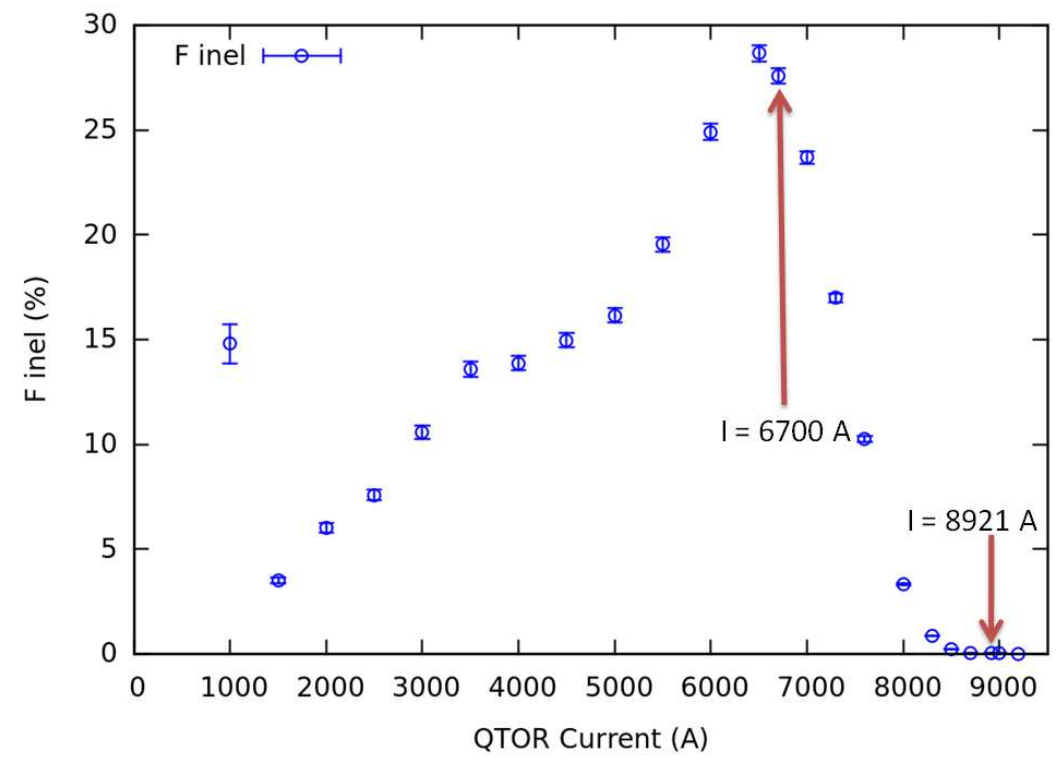

FIG. 5.3: Inelastic dilution from simulation as a function of QTOR setting[13].

The dilution due to inelastic events was determined using simulation[82] to be

$$
f_{\mathrm{b} 4}=0.0002 \pm 0.0002 .
$$

Results from this study found the simulated rates to be $10 \%$ below those determined at the inelastic peak from data. Due to this, a preliminary $100 \%$ error bar was assigned pending further analysis.

\subsubsection{Total Neutral Background}

The neutral background originates from secondary photons and scattering of primary electrons from the collimators and shield walls; a small percentage of the background is made up of pions and neutrons. Estimation of the neutral background was done using the RIII chambers in counting mode; counting mode allows for 
the detection of single events. Neutral events detected in the RIII chambers pass through both the trigger scintillator and the main detectors. Both of these are relatively insensitive to neutral events, however interactions such as neutron capture and Compton scattering, while being below the threshold of detection in the trigger scintillator, may shower in the pre-radiator or interact in the fused silica and cause a signal in the MDs. These events can be classified by the amount of light they produce. In this way, the neutral background fraction is defined by

$$
n_{f}=\frac{M D_{\text {neutral }} \times\left\langle Y_{\text {neutral }}\right\rangle}{M D_{\text {total }} \times\left\langle Y_{\text {total }}\right\rangle} .
$$

Here $M D_{\text {neutral }}$ is the number of detected neutral tracks and $Y_{\text {neutral }}$ is the light yield from those tracks. The neutral fraction is formed as the ratio of this product for neutral tracks versus all tracks. This analysis was done for each detector octant at nominal QTOR current. An in-depth look at this measurement can be found in [5]. The total neutral dilution was found to be

$$
f_{\text {TotalNeutral }}=0.0038 \pm 0.0014(\text { stat. }) \pm 0.0011 \text { (syst.). }
$$

This dilution due to the neutral background is calculated by removing the beamline background dilution from the total neutral background,

$$
\begin{aligned}
& f_{\mathrm{b} 2}=f_{\text {Total Neutral }}-f_{\mathrm{b} 3} \\
& f_{\mathrm{b} 2}=0.00162 \pm 0.00191
\end{aligned}
$$

The neutral background asymmetry is approximated based on the assumption that the source of the neutral background is primarily Møller scattering from scraping on the collimator edges and shield wall apertures. With this in mind, it is 
reasonable to approximate the asymmetry as being 0.00 with an uncertainty on the order of our asymmetry measurement. Therefore a conservative neutral background asymmetry was adopted to be

$$
A_{\mathrm{b} 2}=0.00 \pm 0.20 \mathrm{ppm}
$$

\subsubsection{Experimental Bias Corrections}

Experimental bias encompass corrections due to electromagnetic radiative corrections, detector bias, acceptance, and bias in defining the central $\mathrm{Q}^{2}$. Each of these corrections is applied to the measured asymmetry independently, along with the other corrections listed in this chapter, to determine the physics asymmetry. The first of these, radiative corrections $\left(\mathrm{R}_{R C}\right)$, is due to radiative effects associated with the incident electrons which modify the asymmetry that expected at tree-level. Corrections are made for leading order electromagnetic effects such as Bremsstrahlung and virtual photons both internal and externally to the field of the scattering nucleus. GEANT3 was used to simulate the scattering process both with and without radiative corrections. The experimental bias correction is then defined to be[36]

$$
R_{R C}=\frac{R_{\text {Tree }}}{R_{\mathrm{RC}-\mathrm{Sim}}}=1.0096 \pm 0.0065
$$

The detector bias $\left(R_{\text {det }}\right)$ deals with the light weighting of the main detectors.

Scattered electrons distributed over the $\hat{C}$ erenkov bar produce light, the yield of which is measured and used to compute the measured asymmetry. The light yield however varies spatially over the bar and requires a light weighting correction to accurately compute the asymmetry. Using simulation, the detector bias correction 
is computed as[36]

$$
R_{\text {Det }}=\frac{A_{\text {NoBias }}^{\text {Sim }}}{A_{\text {Bias }}^{\text {Sim }}}=0.987 \pm 0.007
$$

The acceptance bias $\left(R_{\text {Acc }}\right)$ is related to the way in which the asymmetry is extracted with respect to the momentum transfer. The experimental asymmetry measured by experiment is given as $\left\langle A\left(Q^{2}\right)\right\rangle$, however what is needed to compare to theoretical predictions is $A\left(\left\langle Q^{2}\right\rangle\right)$. In order to correct for this, simulation is used to determine the correction given by[36]

$$
R_{\text {Acc }}=\frac{A\left(\left\langle Q^{2}\right\rangle\right)}{\left\langle A\left(Q^{2}\right)\right\rangle}=0.98 \pm 0.01
$$

The final correction $\left(\mathrm{R}_{Q^{2}}\right)$ relates to the uncertainty to which the central $\mathrm{Q}^{2}$ is known[36].

$$
R_{Q^{2}}=1.00 \pm 0.03
$$

Considering all the bias corrections the final correction is applied as

$$
R_{\text {Total }}=R_{\mathrm{RC}} R_{\text {Det }} R_{\text {Acc }} R_{Q^{2}}=0.9765 \pm 0.0323
$$

\subsection{Beam Polarization}

\subsubsection{Møller Polarimetry}

Run I polarization results were computed using the Hall C Møller polarimeter. Discussion of the hardware and operational aspects of the Møller polarimeter can be found in Section 3.3. Møller measurements, which were invasive to regular experimental operation, were carried out at low currents $(1 \mu \mathrm{A})$ on average about three times weekly. Each measurement consisted of three measurements in each half-wave 
plate setting.

One of the more problematic issues of the Run I analysis was due to failures in the $\mathrm{Q}_{3}$ quad. During Run I it was found that this quad exhibited a time-dependent field deterioration which required perpetual retune of the Møller and re-steering of the beam to avoid scraping. The source of the problem was found to be a short in the $\mathrm{Q}_{3}$ quad which reduced the effective magnet current to $\sim 2 / 3$ the nominal value, which in turn affected the analysing power. Currently, it is estimated that the effect on the analysing power could be up to $1.4 \%$. At the moment, work is in progress to determine the total systematic effect which, due to its time dependent nature, must be handled on a case-by-case basis. A list of the current best estimates of systematic errors is shown in Table. 5.1.

The slug average polarization values over Run I can be seen in Fig. 5.4. Given that the polarization over Run I was not stable, the decision was to apply the polarization correction on the slug level; different methods of averaging and applying the polarization were investigated [83] to determine the best approach. The slugaverage polarization values are listed below in Table 5.2. 


\begin{tabular}{|l|c|c|c|}
\hline Source & Error & Scale & pt-to-pt \\
\hline Beam Position X & 0.17 & - & 0.17 \\
Beam Position Y & 0.28 & - & 0.28 \\
Beam Direction X & 0.10 & - & 0.10 \\
Beam Direction Y & 0.10 & - & 0.10 \\
Q1 Current & 0.07 & - & 0.07 \\
Q3 Current & 0.05 & - & 0.05 \\
Q3 Position & 0.10 & 0.10 & - \\
Multiple Scattering & 0.01 & 0.01 & - \\
Levchuk Effect & 0.33 & - & 0.33 \\
Collimator Positions & 0.03 & 0.03 & - \\
Target Temperature & 0.14 & 0.14 & - \\
B-field Direction & 0.14 & 0.14 & - \\
B-field Strength & 0.03 & 0.03 & - \\
Polarization in Fe & 0.25 & 0.25 & - \\
Electronic D.T. & 0.05 & 0.05 & - \\
Solenoid Focusing & 0.21 & 0.21 & - \\
Solenoid Position & 0.23 & 0.16 & 0.16 \\
High Current Extrap. & 0.50 & 0.35 & 0.35 \\
Polfac Nominal Value & 0.65 & 0.65 & - \\
Polfac Variation & 0.23 & - & 0.23 \\
Monte Carlo Stats. & 0.15 & 0.15 & - \\
\hline Total & 1.09 & 0.93 & 0.57 \\
\hline
\end{tabular}

TABLE 5.1: Systematic errors associated with the Møller measurement. 


\begin{tabular}{|c|c|c|}
\hline Slug & Polarization & Error \\
\hline 45 & 0.8826 & 0.0107 \\
\hline 50 & 0.8889 & 0.0107 \\
\hline 53 & 0.8866 & 0.0108 \\
\hline 55 & 0.8815 & 0.0108 \\
\hline 77 & 0.8896 & 0.0106 \\
\hline 90 & 0.8835 & 0.0106 \\
\hline 94 & 0.8799 & 0.0106 \\
\hline 96 & 0.8873 & 0.0108 \\
\hline 98 & 0.8402 & 0.0107 \\
\hline 101 & 0.8597 & 0.0108 \\
\hline 103 & 0.8625 & 0.0106 \\
\hline 105 & 0.8656 & 0.0107 \\
\hline 112 & 0.8563 & 0.0107 \\
\hline 115 & 0.8453 & 0.0107 \\
\hline 117 & 0.8570 & 0.0107 \\
\hline 119 & 0.8541 & 0.0107 \\
\hline 122 & 0.8537 & 0.0108 \\
\hline 124 & 0.8672 & 0.0106 \\
\hline 126 & 0.8677 & 0.0108 \\
\hline 129 & 0.8639 & 0.0108 \\
\hline 132 & 0.8577 & 0.0108 \\
\hline
\end{tabular}

TABLE 5.2: Individual beam polarization measurements during Run I. 


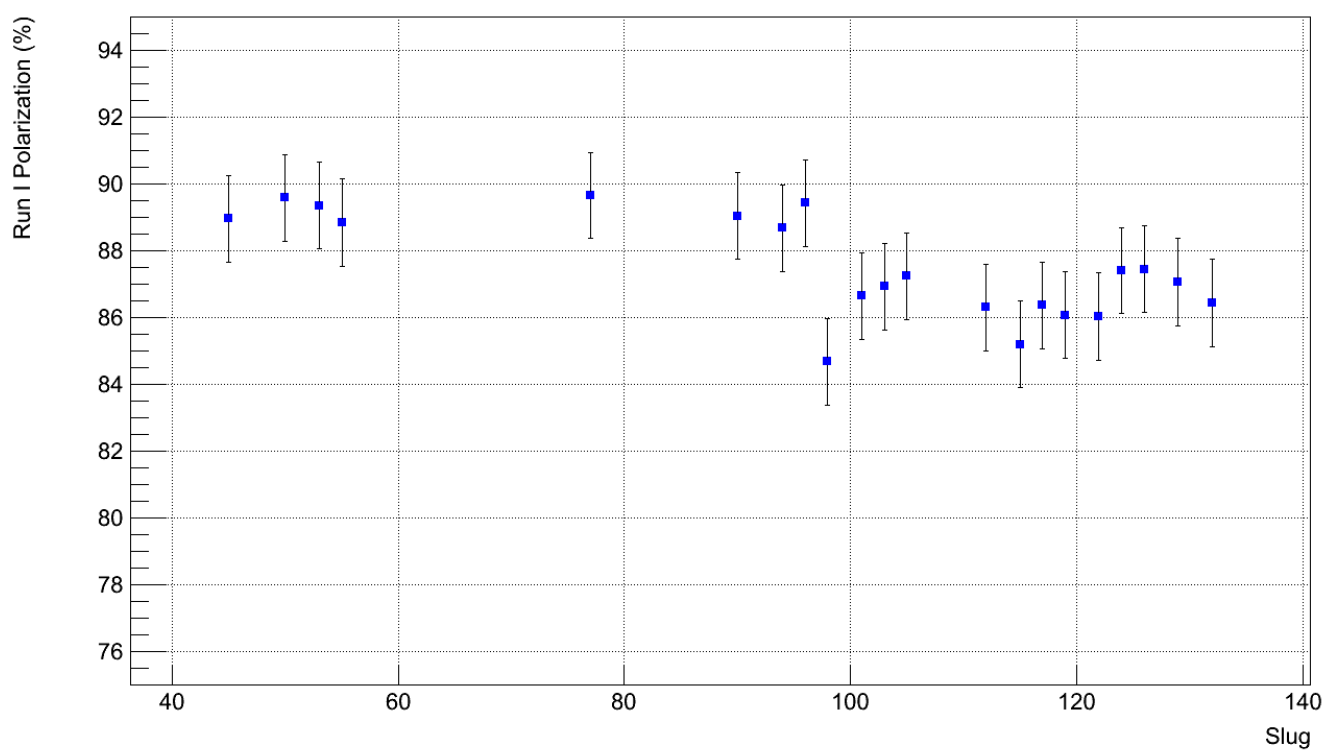

FIG. 5.4: Measured average polarization by slug for Run I data. 


\section{CHAPTER 6}

\section{Results and Discussion}

\section{1 $e+p$ Asymmetry Results}

The following section provides an overview of the calculation of the final asymmetry and extraction of the proton's weak-charge. The raw asymmetry following data quality cuts is given by $\left\langle\mathrm{A}_{\text {raw }}\right\rangle=-243.22 \pm 13.32$ (stat.) ppb. After correction for false asymmetries using the beam modulation sensitivities, the corrected asymmetry is $\left\langle\mathrm{A}_{\mathrm{Reg}}\right\rangle=-232.08 \pm 13.53$ (stat.) ppb. The asymmetry also includes a blinding factor of $\pm 60 \mathrm{ppb}$ which was added to eliminate personal bias from the experimental analysis. The blinding factor was applied in the analyser to each quartet of data. A different blinding factor was used for each run period of the experiment and will only be removed after the physics analysis is finished. This blinding factor was not folded into the error breakdown presented below, but was added in the final determination at the end. As discussed in detail in Sec. 5.2, the asymmetry must be corrected for backgrounds and experimental bias. A summary of the background corrections can be seen in Tab. 6.1 and Fig. 6.1 shows the change in the asymmetry 


\begin{tabular}{|l|c|r|}
\hline Background & Dilution $\left(f_{i}\right)$ & Asymmetry $\left(A_{i}\right)$ ppm \\
\hline Al Window $\left(b_{1}\right)$ & $0.0282 \pm 0.0021$ & $1.1472 \pm 0.0654$ \\
\hline QTOR Transport $\left(b_{2}\right)$ & $0.0016 \pm 0.0019$ & $0.0 \pm 0.2$ \\
\hline Beamline Background $\left(b_{3}\right)$ & $0.0022 \pm 0.0006$ & $-0.0109 \pm 0.0109$ \\
\hline $\mathrm{N} \rightarrow \Delta\left(b_{4}\right)$ & $0.0002 \pm 0.0002$ & $-3.02 \pm 0.97$ \\
\hline
\end{tabular}

TABLE 6.1: The asymmetry and associated dilution for each background source.

after correction using beam modulation and polarization, the size of the background corrections, and the final corrected asymmetry. The corrections were applied to the measured asymmetry after modulation correction according to

$$
A_{\text {physics }}=R_{\text {total }} \frac{\frac{A_{m s r}}{P}-\sum_{i=1}^{n} f_{i} A_{i}}{\left(1-\sum_{i=1}^{n} f_{i}\right)}
$$

where $f_{i}$ and $A_{i}$ are the dilution and asymmetries for each background respectively and $R_{\text {total }}$ is the total experimental bias correction. The asymmetry, $\mathrm{A}_{m s r}$, represents the beam modulation corrected asymmetry and the beam polarization is given by P.

The systematic error contribution from each background is calculated using standard error propagation methods and is shown in detail in Appendix A.3. A breakdown of the error contribution due to each correction, in terms of systematic and statistical uncertainty, is shown in Table 6.2. The corrections are dominated by Aluminium target windows, followed by polarization, and modulation corrections. Corrections due to other background sources are relatively small.

The blinding factor addition to the error of the final asymmetry was computed according to Appendix A.3 to be $68.52 \mathrm{ppb}$. The blinding factor, in this form, represents a uniform distribution of error, however in order to fold it into the total error on the asymmetry it needs to be rewritten in terms of a normal distribution. 


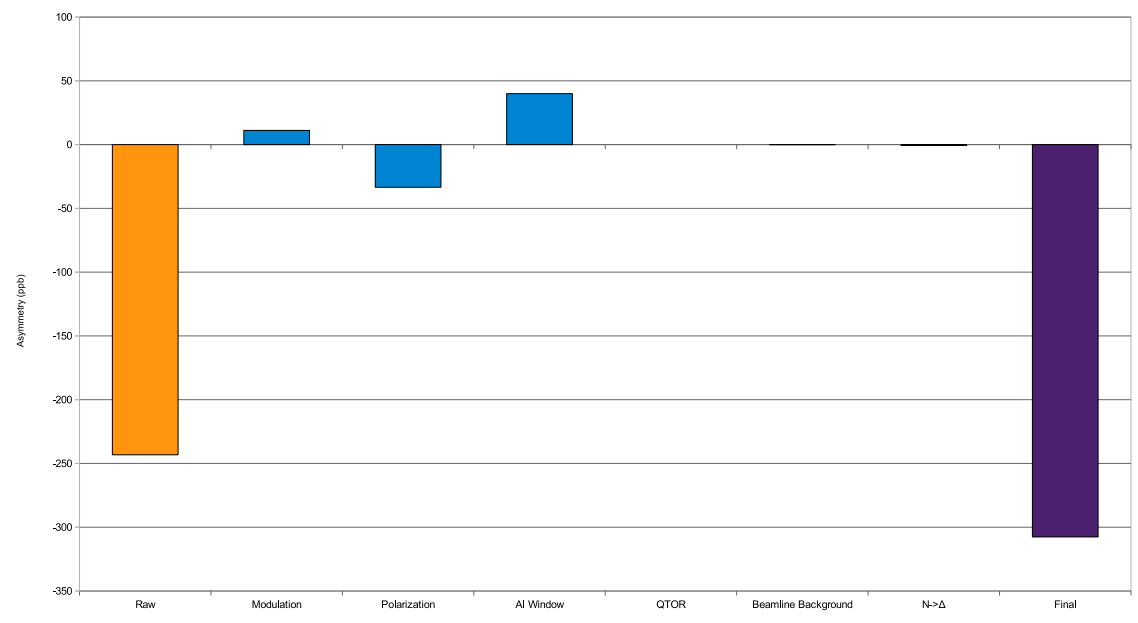

FIG. 6.1: The contribution from various corrections to the measured asymmetry. The raw asymmetry is given on the left in orange. The corrections from beam modulation and background corrections are shown in blue, and the final corrected asymmetry is shown in purple on the far right.

Computing the second moment of the blinding factor gives a value of $39.56 \mathrm{ppb}$, which can be added in quadrature with the systematic and statistical errors. Using $\mathrm{A}_{m s r}$ above as an input to Eq. 6.1 the final physics asymmetry including blinding factor is computed to be

$$
A_{P V}^{e p}\left(\left\langle Q^{2}\right\rangle\right)=-307 \pm 15 \text { (stat.) } \pm 19 \text { (syst.) } \pm 39 \text { (blinding) ppb. }
$$

Using the final asymmetry, the weak charge of the proton as well as the weak vector quark couplings can be extracted. Recalling Eq. 2.22, the physics asymmetry can be written in terms of the electromagnetic and electroweak form factors. The extraction of the weak charge involves fitting this equation as a function of $\mathrm{Q}^{2}$, using global PVES data from [84, 85, 86, 87, 88, 89, 90, 91, 92] on Hydrogen, Helium, and Deuterium targets, using the method outlined in [36]. It is important to note that the 


\begin{tabular}{l|c}
\hline \multicolumn{2}{c}{ Contributed Error } \\
\hline Measured Asymmetry(Syst.) & 15.35 \\
Polarization & 5.32 \\
Aluminium Asymmetry & 5.54 \\
Neutral Background Asymmetry & 0.33 \\
Beamline Background Asymmetry & 0.02 \\
Inelastic Asymmetry & 0.19 \\
Total Experimental Bias (R) & 9.45 \\
Aluminium Dilution & 3.65 \\
Neutral Background Dilution & 0.19 \\
Beamline Background Dilution & 0.19 \\
Inelastic Dilution & 0.53 \\
\hline dA(Syst.) & 19.69 \\
\hline dA(Stat. + Syst.) & 23.89 \\
\hline
\end{tabular}

TABLE 6.2: Breakdown of the systematic errors going into the final uncertainty.

electromagnetic form factors are well determined in terms of global fits [93], which is not the case for the electroweak form factors. Instead, as is shown in Sec.2.2.3, the electroweak form factors can be written in terms of the quark electromagnetic form factors,

$$
G_{E}^{p, Z}=2\left(2 C_{u 1}+C_{d 1}\right) G_{E}^{p, \gamma}+2\left(C_{u 1}+2 C_{d 1}\right) G_{E}^{n, \gamma}+\varepsilon_{V}^{(0)} G_{E}^{s}
$$

where $\varepsilon_{V}^{(0)}$ encompasses contributions from charge symmetry violation [94]. Rewriting the asymmetry in this way, the global fit becomes a function of five independent parameters: the weak vector couplings $\left(C_{1 u}\right.$ and $\left.C_{1 d}\right)$, the strange charge radius $\rho_{s}$, the strange magnetic moment $\mu_{s}$, and the axial form factors $G_{A}^{Z(T=1)}$ and $G_{A}^{Z(T=0)}$. The strange quark form factors are given by [95].

$$
G_{E}^{s}=\rho_{s} Q^{2} G_{D}, \quad G_{M}^{s}=\mu_{s} G_{D}
$$




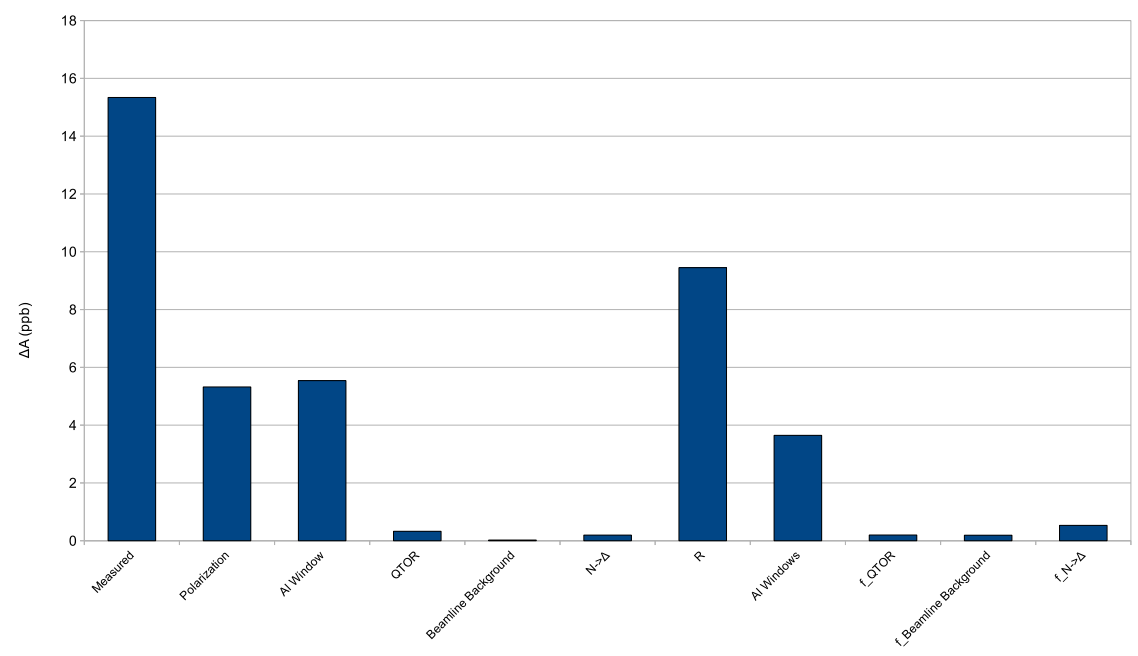

FIG. 6.2: The contributions to the final error bar.

where

$$
G_{D}=\frac{1}{\left(1+\frac{Q^{2}}{\Lambda^{2}}\right)} .
$$

This parametrization was chosen to match the $\mathrm{Q}^{2}$ dependence experimental data and the mass scale $\left(\Lambda^{2}\right)$ was set to $1(\mathrm{GeV} / \mathrm{c})^{2}$ to match PVES data up to $\mathrm{Q}^{2}=$ $0.63(\mathrm{GeV} / \mathrm{c})^{2}[36]$. The axial form factors, separated into isovector $\left(G_{A}^{Z(T=1)}\right)$ and isoscalar $\left(G_{A}^{Z(T=0)}\right)$ terms are constrained by conservative theoretical calculation [95]. All data used in the global fit was corrected for the energy dependence to the $\gamma-Z$ box diagram according to the prescription in Ref.[96]. The associated uncertainties were added in to the systematic error on each data point. The effects of including points at higher $\mathrm{Q}^{2}$ and $\theta$, as well as varying the mass scale $(\Lambda)$ of the dipole parametrization, was studied and the effect were found to be small [36]. Considering the preceding parameters, a standard $\chi^{2}$ minimization is performed which determines the best fit values for each parameter. The resulting equation is used to extrapolate to $\mathrm{Q}^{2}=0$ allowing for extraction of $Q_{w}^{p}(0)$.

While the global fit includes data as a function of $\left(\mathrm{Q}^{2}, \theta\right)$ it does not lend itself 
to interpretability when plotting. For this reason the 2-D fit can be rotated into the forward limit $(\theta=0)$ removing the angular dependence. The rotation was done using the theoretical calculation of the shift in the asymmetry due to the forward limit rotation

$$
\Delta=A_{\text {calc }}\left(\theta, Q^{2}\right)-A_{\text {calc }}\left(0, Q^{2}\right)
$$

This is then used to shift the measured asymmetry $\mathrm{A}_{e p}\left(\theta, Q^{2}\right)$ into the forward limit. The reduced asymmetry can then be defined in the following way

$$
A_{\text {reduce }}=\frac{A_{\mathrm{PV}}}{A_{\circ}}=Q_{W}^{p}+B\left(\theta=0, Q^{2}\right) Q^{2}, A_{\circ}=\left[\frac{\left(-G_{F} Q^{2}\right)}{4 \pi \alpha \sqrt{2})}\right]
$$

This reduced form was used to plot the data points in the global fit as a function of $\mathrm{Q}^{2}$; the extracted value for $\mathrm{Q}_{W}^{p}$ is defined as the intercept of the global fit $\left(\mathrm{Q}^{2} \rightarrow 0\right)$. The extracted values for $\mathrm{Q}_{W}^{p}$ are shown below. Here the results are presented with the blinding factor (blinded) and assuming the blinding factor is approximately zero (Blinding excluded). In the later case the extraction was done without including the error due to blinding.

\begin{tabular}{ll|}
$Q_{W}^{p}($ Blindingexcluded $)$ & $=0.0845 \pm 0.0093$ \\
$Q_{W}^{p}($ Blinded $)$ & $=0.0673 \pm 0.0119$ \\
$Q_{W}^{p}(\mathrm{SM})$ & $=0.0705 \pm 0.0008$.
\end{tabular}

The measured result without the blinding factor is $\approx 1.5 \sigma$ from the SM expectation. The forward angle global fit of the reduced asymmetry is show in Fig. 6.3 assuming the blinding factor is very small or zero.

Using the values of the vector couplings in Table. 2.1, the weak-charge can be written in terms of the quark neutral weak vector couplings as $Q_{W}^{p}=-2\left(2 C_{u 1}+\right.$ 


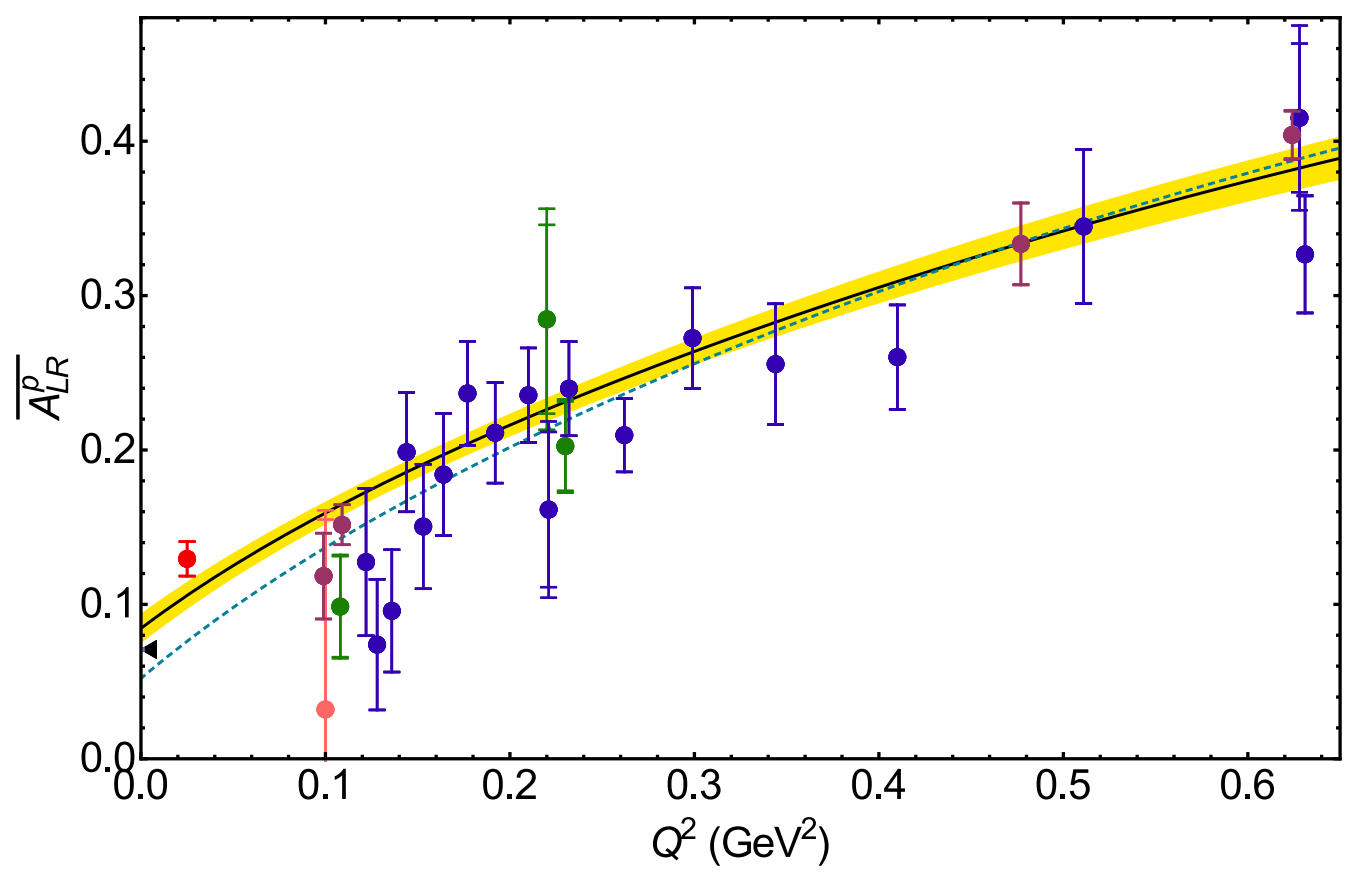

FIG. 6.3: The global fit of the reduced asymmetry in the forward limit to $\mathrm{Q}^{2}=0.63$ $(\mathrm{GeV} / \mathrm{c})^{2}$ is shown as the black line. The red point is the present $Q_{W}^{p}$ measurement. The yellow band represents the uncertainty of the fit. The dashed blue line shows the global fit without the measurement of $\mathrm{Q}_{W}^{p}$ presented here. The SM prediction is shown by the arrow. This fit assumes the blinding factor is close to zero. 
$\left.C_{d 1}\right)$. Both $C_{u 1}$ and $C_{d 1}$ are fit parameters in the global fit used to extract $\mathrm{Q}_{W}^{p}$, however further constraints can be placed on the neutral weak vector couplings by considering results from atomic parity-violation experiments. Recent results [97] from experiments such as [37] on ${ }^{133} \mathrm{Cs}$ provide different linear combinations of the neutral weak vector couplings that can be used to better separate and determine $C_{u 1}$ and $C_{d 1}$. Combining these results with the PVES results gives values of the neutral weak vector couplings of

$C_{u 1}=-0.19286 \pm 0.0042$ (blindingexcluded)
$C_{d 1}=0.34373 \pm 0.0041$ (blindingexcluded)
$C_{u 1}=-0.1851 \pm 0.0054$ (blindingincluded))
$C_{d 1}=0.3368 \pm 0.0050$ (blindingincluded)

The global fit of the quark weak vector couplings can be seen in Fig. 6.4. With the revised number for couplings, the weak-charge was recalculated according to $Q_{W}^{p}=-2\left(2 C_{u 1}+C_{d 1}\right)$. The results of this were found to be

$$
\begin{aligned}
& Q_{W}^{p}=-2\left(2 C_{u 1}+C_{d 1}\right)=0.0839 \pm 0.0092 \text { (blindingexcluded) } \\
& Q_{W}^{p}=-2\left(2 C_{u 1}+C_{d 1}\right)=0.0667 \pm 0.0118 \text { (blindingincluded). }
\end{aligned}
$$

Both the blinded and unblinded values for the calculated $Q_{W}^{p}$ match the corresponding values extracted using the reduced asymmetry above. As with the results from the reduced asymmetry extraction, there is an $\sim 1.5 \sigma$ discrepancy between the measured and SM prediction for the physics asymmetry. These results must be taken in context though. While the results presented above do correct for the blinding factor, it is still included in the data and must be considered. Presenting the values without the blinding factor was done to show what the results would be if 


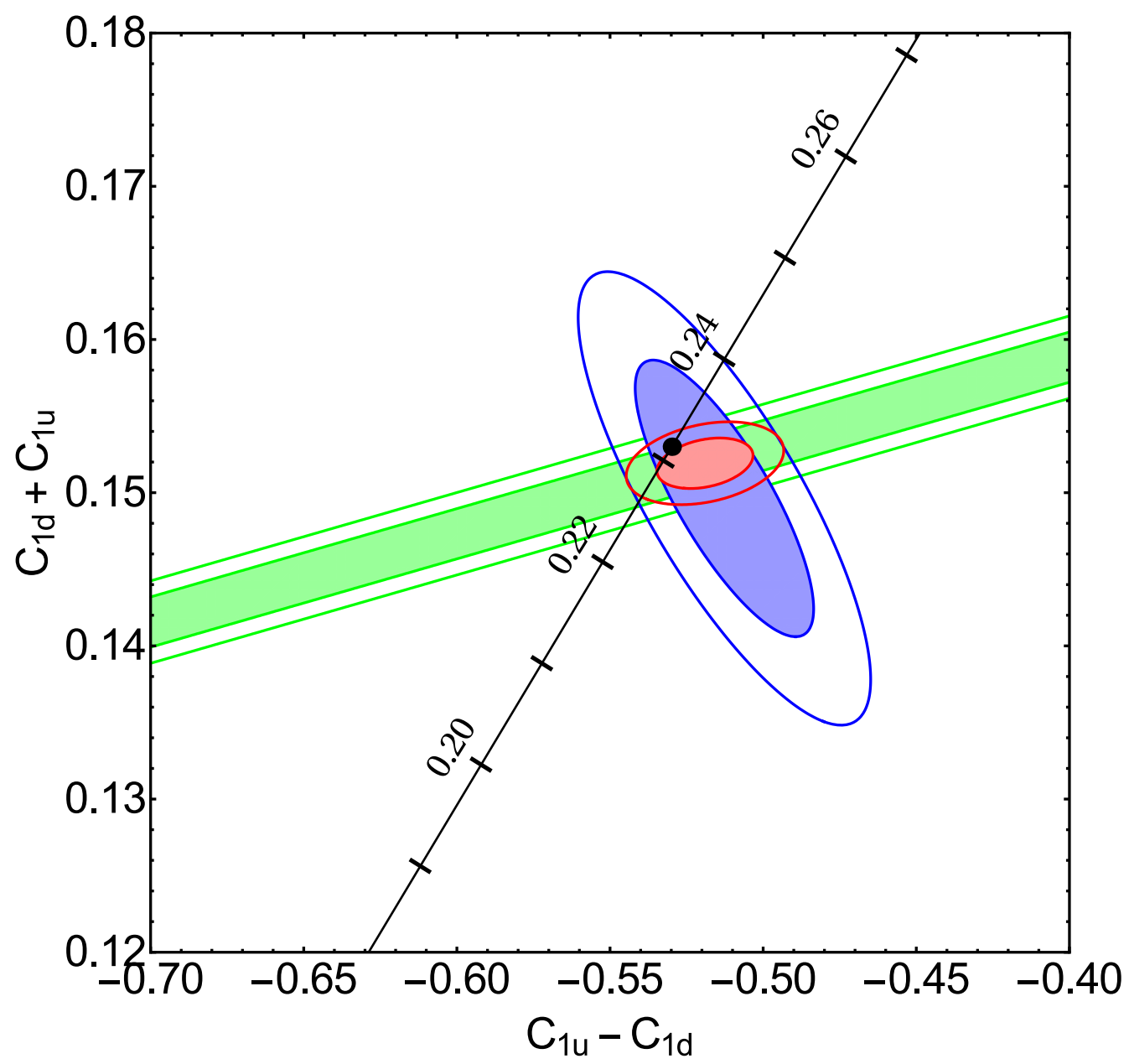

FIG. 6.4: Constraints on the neutral weak vector constants: isoscalar $\left(C_{u 1}+C_{d 1}\right)$ and isovector $\left(C_{u 1}-C_{d 1}\right)$. The APV measurement shown in green mainly constrains the isoscalar combination. The blue ellipse includes the current PVES data up to $\mathrm{Q}^{2}<0.63(\mathrm{GeV} / \mathrm{c})^{2}$ as well as the value reported here. The red ellipse is the combination of PVES and APV measurements; inner ellipses are $68 \%$ confidence level and the outer are $95 \%$ confidence level. The black line represents the $\overline{M S}$ scheme SM prediction of $\sin ^{2} \theta_{W}$ [14], with the black dot indicating the SM best fit value, $\sin ^{2} \theta_{W}=0.23116$. 
the blinding factor ends up being small. Because of this, drawing scientific conclusions from these results is difficult. The complete analysis of the Run I dataset is on-going. Following its completion, and subsequent blinding factor removal, more concrete conclusions will be possible.

\subsection{Conclusion \& Future Work}

While the results presented here represent a factor of two improvement in precision over the original Wien 0 result, there is still a lot of work to be done. The Run I data set makes up approximately $33 \%$ of the total $Q_{w e a k}$ data set (in comparison to the $4 \%$ from Wien 0 ), but completion of the Run II data analysis, which makes up $63 \%$ of the total data set, should see yet more improvement of the statistical error. The bulk of the systematic error comes from uncertainties on the Aluminium measurements, $\mathrm{Q}^{2}$, and polarization. Work is currently in progress to improve the uncertainties of the Aluminium measurement by considering higher order diagrams, radiative losses, and better understanding of the beamline backgrounds which contribute heavily to the uncertainty of the Aluminium asymmetry. Work on the $\mathrm{Q}^{2}$ measurement is on-going but has been primarily focused on the Run II analysis thus far. Another source, which has recently been revisited, is the polarization determination. The issues with the failed coil during Run I have spawned a substantial amount of simulation and analysis effort by the polarization group. Given that this is a subtle issue, the effect of which was changing daily during Run I, and man-power is low, the progress on this has been slow, however, with the shift in focus of the analysis changing to Run I, more results are expected in the near future.

In terms of work on improving the determination of the beam modulation extracted detector sensitivities, a substantial amount of work has been done. Many 
of the issues presented here in the Run I analysis have been rectified. The gaps in the ramp function have been fixed, the number of independent variables has been doubled - both the sine and cosine contributions are now considered - and the BPMs been redefined as "effective" BPMs which have provided much better separation among the BPM responses. In general the current analysis efforts have been focused heavily on the Run II data set and only recently has the focus started to shift towards Run I; it is expected that this will greatly reduce the systematic errors as a whole as the analysis ramps up. I have included a more detail write-up with proposed changes to a future beam modulation system in App. A.4. 


\section{BIBLIOGRAPHY}

[1] Wikimedia Commons. Standard model of elementary particle physics. https://commons.wikimedia.org/wiki/Standard_Model_of_Elementary_ Particles.svg, 2006.

[2] Jens Erler and Michael J. Ramsey-Musolf. Weak mixing angle at low energies. Phys. Rev. D, 72:073003, 2005.

[3] Daniel T. Pierce and F. Meier. Negative electron affinity gaas: A new source of spin-polarized electrons. Applied Physics Letters, Vol.26(12):670-672, 1975.

[4] K. D. Paschke. Controlling helicity-correlated beam asymmetries in a polarized electron source. Eur. Phys. J. A., 32:549-553, 2006.

[5] Rakitha Beminiwattha. A Measurement of the Weak Charge of the Electron through Parity Violating Electron Scattering. PhD. Thesis, Ohio University, 2013.

[6] Waidyawansa Buddhini. Beam Position and Angle Determination in Qweak. Qweak Document Database(Qweak-Doc-1772-v2), 2012.

[7] Richard Jones. Polarimetry for Qweak. Qweak Document: Qweak-doc-510-v1, 2005. 
[8] K.E. Myers. The First Determination of the Proton's Weak Charge Through Parity-Violating Asymmetry Measurements in Elastic e+p and e+Al Scattering. PhD Thesis, George Washington University, 2012.

[9] K.E. Myers. GEANT3 Simulation of Detector Shielding Wall Design Technical Report. Qweak Document Qweak-doc-852-v1, 2008.

[10] John P. Leckey IV. The First Direct Measurement of the Weak Charge of the Proton. PhD Thesis, The College of William and Mary, 2012.

[11] Nuruzzaman. Beam Modulation Cable Map for Q-weak experiment in Hall-C at Jefferson Lab. Qweak Document: Qweak-doc-1279-v2, 2010.

[12] Nuruzzaman. $1^{\text {st }}$ Order Beam Modulation in the 3C Line for Qweak experiment. Qweak Document: Qweak-doc-910-v3, 2009.

[13] J. Leacock. Measuring the Weak-Charge of the Proton and the Hadronic ParityViolation of the $N \rightarrow \Delta$ Transition. PhD. Thesis, Virginia Polytechnic Institute and State University, 2012.

[14] J. Beringer et al. Review of particle physics*. Phys. Rev. D, 86:010001, 2012.

[15] E. Noether. Invariante Variationsprobleme. Nachr. D. Knig. Gesellsch. D. Wiss. Zu Gttingen, Math-phys., Klasse:235-257, 1918.

[16] E. D. Bloom, D. H. Coward, H. Destaebler, J. Drees, G. Miller, L. W. Mo, R. E. Taylor, M. Breidenbach, J. I. Friedman, G. C. Hartmann, and H. W. Kendall. High-Energy Inelastic e-p Scattering at $6^{\circ}$ and $10^{\circ}$. Physical Review Letters, 23:930-934, 1969. 
[17] M. Breidenbach, J. I. Friedman, H. W. Kendall, E. D. Bloom, D. H. Coward, H. Destaebler, J. Drees, L. W. Mo, and R. E. Taylor. Observed Behavior of Highly Inelastic Electron-Proton Scattering. Physical Review Letters, 23:935939, 1969.

[18] S. L. Glashow. Partial-symmetries of weak interactions. , 22:579-588, 1961.

[19] P. W. Higgs. Broken Symmetries and the Masses of Gauge Bosons. Physical Review Letters, 13:508-509, 1964.

[20] S. Weinberg. A Model of Leptons. Physical Review Letters, 19:1264-1266, 1967.

[21] A. Salam. Elementary Particle Physics: Relativistic Groups and Analyticity. Eighth Nobel Symposium. Stockholm: Almquvist and Wiksell, 1968.

[22] M. Gell-Mann. A schematic model of baryons and mesons. Physics Letters, $8: 214-215,1964$.

[23] G. Zweig. An SU(3) Model for Strong Interaction Symmetry and its Breaking. CERN Report No.8182/TH.401, 1964.

[24] Hasert F. J. et al. Observation of neutrino-like interactions without muon or electron in the gargamelle neutrino experiment. Physics Letters B, 46:138-140, 1973.

[25] F. J. Hasert et al. Search for elastic muon-neutrino electron scattering. Physics Letters B, 46:121-124, 1973.

[26] C.Y. Prescott et al. Parity Nonconservation in Inelastic Electron Scattering. Physics Letters, pages 347-352, 1978. 
[27] G. Arnison et al. Experimental Observation of Isolated Large Transverse Energy Electrons with Associated Missing Energy at $\sqrt{s}=540 \mathrm{GeV}$. Physics Letters, pages 103-116, 1983.

[28] M. Banner et al. Observation of Single Isolated Electrons of High Transverse Momentum in Events with Missing Transverse Energy at the CERN $\bar{p} p$ Collider. Physics Letters, pages 476-485, 1983.

[29] G. Arnison et al. Experimental Observation of Lepton Pairs of Invariant Mass Around $95(\mathrm{GeV} / \mathrm{c} 2)$ at the CERN SPS Collider. Physics Letters, pages 398-410, 1983.

[30] P. Bagnaia et al. Evidence of the $Z^{\circ} \rightarrow e^{+} e^{-}$at CERN $\bar{p} p$ Collider. Physics Letters, pages 130-140, 1983.

[31] S. Chatrchyan et al. Observation of a new boson at a mass of $125 \mathrm{GeV}$ with the CMS experiment at the LHC. Physics Letters, pages 30-61, 2012.

[32] G. Aad et al. Observation of a new particle in the search for the Standard Model Higgs boson with the ATLAS detector at the LHC. Physics Letters, pages 1-29, 2012.

[33] T. D. Lee and C. N. Yang. Question of Parity Conservation in Weak Interactions. Physical Review, 104:254-258, 1956.

[34] C. S. Wu, E. Ambler, R. W. Hayward, D. D. Hoppes, and R. P. Hudson. Experimental Test of Parity Conservation in Beta Decay. Physical Review, 105:1413-1415, 1957.

[35] J. Erler and M.J. Ramsey-Musolf. Low Energy Tests of the Standard Model. Prog. Part. Nucl. Phys., 54:351, 2005. 
[36] D. Androic et al. First detrmination of the weak charge of the proton. Phys. Rev. Lett., 111:141803, 2013.

[37] C.S. Wood et al. Measurement of Parity Nonconservation and an Anapole Moment in Cesium. Science, 275:1759-1763, 1997.

[38] A. Sirlin A. Ferroglia, G. Ossola. The electroweak form factor $\hat{\kappa}\left(q^{2}\right)$ and the running of $\sin ^{2} \theta_{w}$. The European Physical Journal C-Particles and Fields., 34:165-171, 2004.

[39] B. Rislow. Low Energy Tests of the Standard Model. PhD. Thesis, College of William and Mary, 2013.

[40] Benjamin C. Rislow and Carl E. Carlson. Contributions from $\gamma Z$ box diagrams to parity violating elastic ep scattering. Phys.Rev., D83:113007, 2011.

[41] A. Sibirtsev, P. G. Blunden, W. Melnitchouk, and A. W. Thomas. $\gamma z$ corrections to forward-angle parity-violating ep scattering. Phys. Rev. D, 82:013011, 2010.

[42] M. Gorchtein, C.J. Horowitz, and M.J. Ramsey-Musolf. Model-dependence of the gamma-Z dispersion correction to the parity-violating elastic ep asymmetry. Phys. Rev. C, 84:015502, 2011.

[43] N.L. Hall, P.G. Blunden, W. Melnitchouk, A.W. Thomas and R.D. Young. Constrained Z interference corrections to parity-violating electron scattering. Phys. Rev. D, page 013011, 2013.

[44] D. Wang et al. Measurements of Parity-Violating Asymmetries in ElectronDeuteron Scattering in the Nucleon Resonance Region. Phys. Rev. Lett., 111:082501, 2013. 
[45] D.S. Armstrong et al. Strange Quark contributions to Parity Violating Asymmetries in Forward G0 Electron-Proton Scattering Experiment. Physical Review Letters, 95:092001, 2005.

[46] K.A. Anilol et al. Parity Violating Electron Scattering from He-4 and Strange Electric Form-Factor of the Nucleon. Physical Review Letters, 96:022003, 2006.

[47] H.A. Grunder, J.J. Bisognano, W.I. Diamond, B.K. Hartline, C.W. Leemann, et al. The Continuous Electron Beam Accelerator Facility. Nucl.Phys., A478:831C-846C, 1988.

[48] Y. C. Chao and others. CEBAF Accelerator Achivements. J. Phys. Conf. Ser., 299:1-18, 2011.

[49] G. D. Cates. Overview of laser systematics. Eur. Phys. J. A., 24:109-114, 2005.

[50] P.A. Adderley and others. Two Wien Filter Spin Flipper. 2011 Particle Accelerator Conference Proceedings, C110328:862-864, 2011.

[51] J.M. Grames et al. Unique electron polarimeter analyzing power comparison and precision spin-based energy measurement. Phys.Rev.ST Accel.Beams, 7:042802, 2004 .

[52] A. S. Hofler et al. Performance of the CEBAF arc beam position monitors. 1993 IEEE Particle Accelerator Conference(PAC 93), May, 17-20 1993.

[53] J. Bergoz and others. Current monitors for particle beams. Nuclear Physics A, 525:595-600, 1991.

[54] M. Hauger et al. A High-Precision Polarimeter. Nucl. Instrum. Meth., A462:382-392, 2001. 
[55] R.A. Reck and D.L. Fry. Orbital and Spin Magnetization in Fe-Co, Fe-Ni, and Ni-Co. Physical Review, 184(2):492, 1969.

[56] G. Smith S. Covrig and J. Dunne. Qweak Liquid Hydrogen Target: Preliminary Design and Safety Document. Qweak Document: Qweak-doc-1041-v2, 2009.

[57] W. Faulk D. Jones L. Lee and P. Wang. QTOR Magnetic Mapping. Collaboration Meeting Talk. Qweak Document: Qweak-doc-1240, 2010.

[58] P. Wang. A Measurement of the Proton's Weak Charge using an Integration Čerenkov Detector System. PhD Thesis, The University of Manitoba, 2011.

[59] D. Mack. Status of the Qweak Main Detector Technical Report. Qweak Document Qweak-doc-649-v1, 2007.

[60] Rob Veenhof CERN. Garfield simulation of gaseous detectors. http:// garfield.web.cern.ch/garfield/, 2010. [Online; accessed 28-February2014].

[61] David Armstrong. Summary of Region 3 Surveys. http://dilbert.physics. wm. edu/Commissioning/63, 2015.

[62] Nuruzzaman. A Robust Beam Modulation Strategy for the Hall-C Beamline. Qweak Document: Qweak-doc-1038-v1, 2009.

[63] Valeri Lebedev. Computer code for linear and non-linear optics calculations. http://www-ap.fnal.gov/ ostiguy/OptiM/, 2014.

[64] D. Jones. Update on Run 2 Beam Modulation. Qweak Document: Qweak-doc1856-v1, 2013. 
[65] Brian J. Moffit. Elastic Scattering of Longitudinally Polarized Electrons from ${ }^{4}$ He. PhD. Thesis, College of William and Mary, 2007.

[66] K. Saenboonruang. Measurement of the Neutron Radius of ${ }^{208} \mathrm{~Pb}$ Through Parity Violating Electron Scattering. PhD. Thesis, University of Virginia, 2008.

[67] J. Hoskins. Study of the Effect of FFB on BPM Response During Modulation. Qweak Document: Qweak-doc-2008-v1, 2014.

[68] K. Paschke. Notes on BPM Phase Shift Due to FFB in the Modulation System. Qweak Document: Qweak-doc-2007-v1, 2012.

[69] D. Jones. Update on Run II Beam Modulation. Qweak Document: Qweakdoc-1856-v1, 2013.

[70] M. Dalton. How neutral is your axis? https://qweak.jlab.org/elog/ Analysis+\%26+Simulation/431, 2011.

[71] C. Yan, P. Adderley, D. Barker, J. Beaufait, K. Capek, R. Carlini, J. Dahlberg, E. Feldl, K. Jordan, B. Kross, W. Oren, R. Wojcik, and J. VanDyke. Superharp a wire scanner with absolute position readout for beam energy measurement at CEBAF. Nuclear Instruments and Methods in Physics Research Section A: Accelerators, Spectrometers, Detectors and Associated Equipment, 365(23):261$267,1995$.

[72] C. Yan, R. D. Carlini, and D. Neuffer. Beam Energy Measurement using Hall C Beamline. Proc. Particle Accelerator Conference, page 2136.

[73] K. A. Aniol et al. Parity-violating electroweak asymmetry in $\overrightarrow{e p}$ scattering. Phys. Rev. C, 69:065501, 2004. 
[74] K. Myers. Pass5 Round 1 Asymmetry for Wien 0 Aluminum Including Systematics. https://qweak.jlab.org/elog/Analysis+\%26+Simulation/743, 2012.

[75] D. Mack. Input file v3(frozen!) for Wien0 4\% DS Al corrected asymmetry. https://qweak.jlab.org/elog/Analysis+\%26+Simulation/753, 2012.

[76] K. Myers. Radiatively Corrected Asymmetry for Wien 0 Pass5 Aluminum. https://qweak.jlab.org/elog/Analysis+\%26+Simulation/748, 2012.

[77] J. Magee. The Preliminary Pluminium Window Dilution for Run I. https: //qweak.jlab.org/doc-private/ShowDocument?docid=1781, 2013.

[78] J. Magee. Corrected 25\% Aluminum Dilution. https://qweak.jlab.org/ elog/Analysis+\%26+Simulation/744, 2012.

[79] Wade Duvall. Fractional yields in blocked MD's in good halo conditions. https : //qweak.jlab.org/elog/Analysis+\%26+Simulation/779, 2012.

[80] Dave Mack. Wien 0 Beamline Background Dilution Central Value and Uncertainty. https://qweak.jlab.org/elog/Analysis+\%26+Simulation/784, 2012.

[81] Wade Duvall and Mark Pitt. Beamline background asymmetry for Wien 0 analysis. https://qweak.jlab.org/elog/Analysis+\%26+Simulation/780, 2012.

[82] A. Subebi. QWGEANT 3 simulations of rates inelastic dilution factor. https: //qweak.jlab.org/elog/Analysis+\%26+Simulation/451, 2011.

[83] J. Hoskins. Beam Polarization Averaging Study. https://qweak.jlab.org/ elog/Analysis+\%26+Simulation/1400, 2015. 
[84] K. A. Aniol et al. Measurement of the neutral weak form factors of the proton. Phys. Rev. Lett., 82:1096-1100, 1999.

[85] K. A. Aniol et al. Parity-violating electron scattering from ${ }^{4} \mathrm{He}$ and the strange electric form factor of the nucleon. Phys. Rev. Lett., 96:022003, 2006.

[86] K.A. Aniol et al. Constraints on the nucleon strange form-factors at $\mathrm{Q}^{* * 2}$ 0.1GeV**2. Phys.Lett., B635:275-279, 2006.

[87] A. Acha et al. Precision measurements of the nucleon strange form factors at $Q^{2} \sim 0.1$ gev $^{2}$. Phys. Rev. Lett., 98:032301, 2007.

[88] Z. Ahmed et al. New precision limit on the strange vector form factors of the proton. Phys. Rev. Lett., 108:102001, 2012.

[89] D. S. Armstrong et al. Strange-quark contributions to parity-violating asymmetries in the forward g0 electron-proton scattering experiment. Phys. Rev. Lett., 95:092001, 2005.

[90] D. Androic et al. Strange quark contributions to parity-violating asymmetries in the backward angle g0 electron scattering experiment. Phys. Rev. Lett., 104:012001, 2010.

[91] F. E. Maas et al. Measurement of strange-quark contributions to the nucleon's form factors at $Q^{2}=0.230(\mathrm{GeV} / c)^{2}$. Phys. Rev. Lett., 93:022002, 2004.

[92] F. E. Maas et al. Evidence for strange-quark contributions to the nucleon's form factors at $Q^{2}=0.108(\mathrm{GeV} / c)^{2}$. Phys. Rev. Lett., 94:152001, 2005.

[93] J. J. Kelly. Simple parametrization of nucleon form factors. Phys. Rev. C, 70:068202, 2004. 
[94] S. MacEwan. The Weak Charge of the Proton: A Search For Physics Beyond the Standard Model. PhD. Thesis, University of Manitoba, 2015.

[95] Shi-Lin Zhu, S. J. Puglia, B. R. Holstein, and M. J. Ramsey-Musolf. Nucleon anapole moment and parity-violating ep scattering. Phys. Rev. D, 62:033008, 2000 .

[96] Mikhail Gorchtein, C.J. Horowitz, and Michael J. Ramsey-Musolf. Modeldependence of the $\gamma Z$ dispersion correction to the parity-violating asymmetry in elastic ep scattering. Phys.Rev., C84:015502, 2011.

[97] V. A. Dzuba, J. C. Berengut, V. V. Flambaum, and B. Roberts. Revisiting parity nonconservation in cesium. Phys. Rev. Lett., 109:203003, 2012.

[98] D. Keith Robinson P. R. Bevington. Data Reduction and Error Analysis. McGraw-Hill, 3rd edition, 2003. 


\section{APPENDIX A}

\section{Appendix}

\section{A.1 Detector Error Weighting}

The weighted average of a variable $x$ is given by,

$$
\langle x\rangle=\frac{\sum_{i}^{N} x_{i} w_{i}}{\sum_{i}^{N} w_{i}},
$$

where $w_{i}$ is a general weight. The variance of $\langle x\rangle$ is given by the error propagation formula [98],

$$
\sigma_{\langle x\rangle}^{2}=\sum_{i}^{N}\left(\frac{\partial\langle x\rangle}{\partial x_{i}}\right)^{2} \sigma_{i}^{2}
$$


Applying Eq. A.2 to Eq. A.1,

$$
\begin{aligned}
& \sigma_{\langle x\rangle}^{2}=\sum_{i}^{N}\left(\frac{w_{i} \sigma_{i}}{\sum_{j}^{N} w_{j}}\right)^{2} \\
& \sigma_{\langle x\rangle}=\sqrt{\sum_{i}^{N}\left(\frac{w_{i} \sigma_{i}}{\sum_{j}^{N} w_{j}}\right)^{2}}
\end{aligned}
$$

we obtain the general form of the variance on the weighted average of a variable $x$ with weight $w_{i}$. Defining the weighting factor to be the main detector error, $w_{i}=1 / \sigma_{M D}^{2}$ we obtain the error on the detector weighted average of $x$.

$$
\sigma_{\langle x\rangle}=\sqrt{\sum_{i}^{N}\left(\frac{\frac{\sigma_{i}}{\sigma_{i, M D}^{2}}}{\sum_{j}^{N} \frac{1}{\sigma_{j, M D}^{2}}}\right)^{2}}
$$




\section{A.2 Rotator Study}

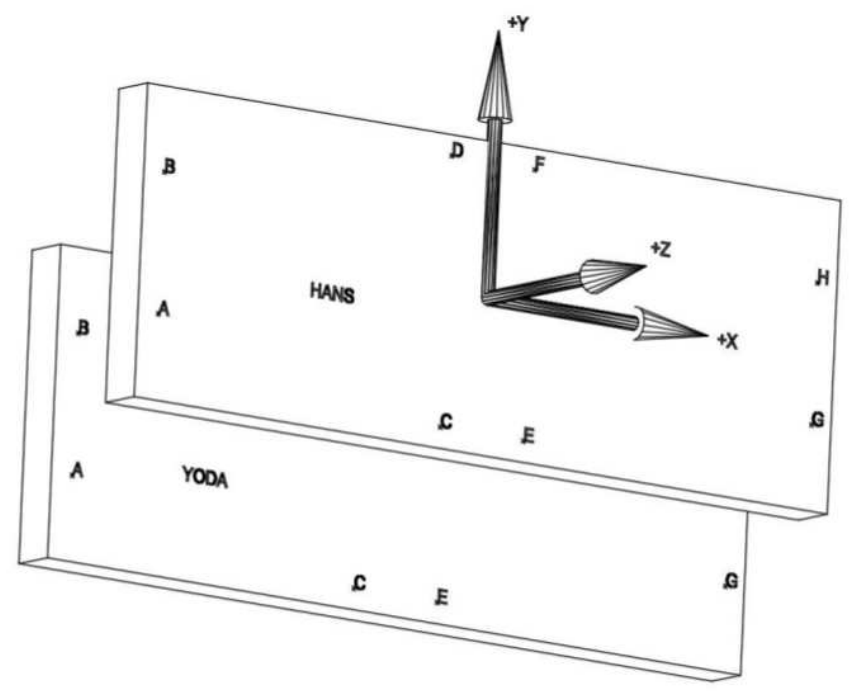

FIG. A.1: The survey team used tooling balls that are attached in static locations on the VDCs to determine position in the frame of the lab. Above shows the positions and designation of each tooling ball.

During and before the running of $\mathrm{Q}_{w e a k}$ a number of surveys were performed to analyze the quality of the motion and repeatability of the Region III rotator. The results of this study were derived from a survey done in September of 2010. The two main items of interest from the study were the rotational and angular repeatability. The survey was done using tooling balls which were attached statically to the face of the VDCs and were used to determine the position with respect to the beamline in the reference frame of the hall. Placement locations of the tooling balls can be seen in Fig. A.1.

The numbers shown in Tab. A.1- A.2 are for moving the rotator from home (defined to be horizontal or at $0^{\circ}$ ) to approximately $45^{\circ}$ and back to home. The listed value is the angle between the $\vec{R}(x, y, z)$ and $\vec{R}^{\prime}(x, y, z)$ vectors which describe the radial position before and after rotation. The angle was computed using the 


\begin{tabular}{|l|l|l|l|l|}
\hline \multicolumn{5}{|c|}{ Vader $^{\circ}$} \\
\hline Point & Angle Repeat 1 & Radial Repeat & Angle Repeat 2 & Radial Repeat 2 \\
\hline VAH & 1.002 & 0.160 & 2.755 & 0.291 \\
\hline VBH & 1.197 & 0.134 & 2.534 & 0.269 \\
\hline VCH & 2.690 & 0.294 & 2.105 & 0.235 \\
\hline VDH & 1.685 & 0.307 & 2.495 & 0.265 \\
\hline VEH & 2.063 & 0.352 & 2.454 & 0.262 \\
\hline VFH & 2.990 & 0.337 & 2.115 & 0.233 \\
\hline VGH & 3.735 & 0.488 & 2.477 & 0.258 \\
\hline VHH & 3.278 & 0.492 & 2.443 & 0.260 \\
\hline
\end{tabular}

TABLE A.1: Angular and radial repeatability values measured after moving from HOME $\rightarrow 45^{\circ} \rightarrow$ HOME. Radial values are shown in millimeters and angular values are in milli-degrees.

\begin{tabular}{|c|l|l|}
\hline \multicolumn{3}{|c|}{ Yoda $^{\circ}$} \\
\hline Point & Angle Repeat & Radial Repeat \\
\hline YAH & 2.705 & 0.313 \\
\hline YBH & 2.629 & 0.330 \\
\hline YCH & 3.631 & 0.340 \\
\hline YDH & 3.252 & 0.365 \\
\hline YEH & 3.863 & 0.354 \\
\hline YFH & 3.440 & 0.375 \\
\hline YGH & 4.452 & 0.413 \\
\hline YHH & 4.162 & 0.418 \\
\hline
\end{tabular}

TABLE A.2: Angular and radial repeatability values measured after moving from HOME $\rightarrow 45^{\circ} \rightarrow$ HOME. Radial values are shown in millimeters and angular values are in milli-degrees. 


\begin{tabular}{|l|l|l|l|l|l|}
\hline \multicolumn{7}{|c|}{ Vader Radial Repeatability } \\
\hline Point & $\delta \mathrm{Z}$ & $\delta \mathrm{X}$ & $\delta \mathrm{Y}$ & $\delta \mathrm{R}(\mathrm{x}, \mathrm{y}, \mathrm{z})$ & $\delta \mathrm{R}(\mathrm{x}, \mathrm{y})$ \\
\hline VAH & 0.09 & -3.53 & 0.29 & 3.543 & 3.387 \\
\hline VBH & 0.04 & -3.55 & 0.67 & 3.613 & 3.507 \\
\hline VCH & 0.02 & -2.54 & 0.85 & 2.685 & 2.588 \\
\hline VDH & 0.11 & -2.49 & 0.11 & 2.495 & 2.492 \\
\hline VEH & 0.10 & -2.2 & 0.11 & 2.205 & 2.192 \\
\hline VFH & 0.02 & -2.24 & 0.85 & 2.396 & 2.183 \\
\hline VGH & 0.03 & -1.21 & 0.67 & 1.383 & 0.820 \\
\hline VHH & 0.08 & -1.19 & 0.29 & 1.227 & 0.997 \\
\hline
\end{tabular}

TABLE A.3: Radial repeatability after moving the chambers from the extended (in beam) location to the retracted (out of beam) and back. All values are given in millimeters.

\begin{tabular}{|l|l|l|l|l|l|}
\hline \multicolumn{7}{|c|}{ Yoda Radial Repeatability } \\
\hline Point & $\delta \mathrm{Z}$ & $\delta \mathrm{X}$ & $\delta \mathrm{Y}$ & $\delta \mathrm{R}(\mathrm{x}, \mathrm{y}, \mathrm{z})$ & $\delta \mathrm{R}(\mathrm{x}, \mathrm{y})$ \\
\hline VAH & 0.04 & 0.06 & -0.55 & 0.555 & 0.553 \\
\hline VBH & -0.01 & 0.08 & -0.63 & 0.635 & 0.635 \\
\hline VCH & 0.01 & -0.14 & -0.51 & 0.529 & 0.529 \\
\hline VDH & -0.08 & -0.10 & -0.67 & 0.682 & 0.677 \\
\hline VEH & 0.00 & -0.20 & -0.51 & 0.548 & 0.548 \\
\hline VFH & -0.10 & -0.15 & -0.67 & 0.694 & 0.687 \\
\hline VGH & -0.07 & -0.38 & -0.55 & 0.672 & 0.669 \\
\hline VHH & -0.12 & -0.36 & -0.63 & 0.735 & 0.726 \\
\hline
\end{tabular}

TABLE A.4: Radial repeatability after moving the chambers from the extended (in beam) location to the retracted (out of beam) and back. All values are given in millimeters.

Law of Cosines for $\vec{R}$ and $\vec{R}^{\prime}$. The Radial repeat is defined as $\delta r=\vec{R}-\vec{R}^{\prime}$ or the difference in the radial vectors before and after rotation. This details how static the radial position of the VDC was after a set of rotations. Some values which are missing were not measured for both VDC packages due to time constraints.

Tables A.3 adn A.4 are the difference in the measured radius of each tooling ball after the rotator has been retracted and re-extended. You will notice a significant difference in the size of the $\delta \mathrm{x}$ value for the measurement of Vader. We have 


\begin{tabular}{|l|l|}
\hline \multicolumn{2}{|c|}{ Vader - $45^{\circ}$} \\
\hline Point & Measured Angle \\
\hline VAH & 45.143 \\
\hline VBH & 45.146 \\
\hline VCH & 45.116 \\
\hline VDH & 45.120 \\
\hline VEH & 45.113 \\
\hline VFH & 45.106 \\
\hline VGH & 45.089 \\
\hline VHH & 45.094 \\
\hline
\end{tabular}

TABLE A.5: Shown is the extracted angle with respect to the home position.

\begin{tabular}{|l|l|}
\hline \multicolumn{2}{|c|}{ Yoda $-45^{\circ}$} \\
\hline Point & Measured Angle \\
\hline VAH & N/A \\
\hline VBH & N/A \\
\hline VCH & N/A \\
\hline VDH & N/A \\
\hline VEH & N/A \\
\hline VFH & N/A \\
\hline VGH & N/A \\
\hline VHH & N/A \\
\hline
\end{tabular}

TABLE A.6: Unfortunately we did not get survey locations for all points on each chamber. This table is listed for completeness.

attributed to incorrect the seating of the linear pin which defines the linear position of the chambers and holds them in place. For this reason, the radial repeatability was based off of the Yoda package. The repeatability is listed both with and without the contribution from the $\mathrm{Z}$ degree of freedom.

Tables A.7 to A.14 present the measured angle between the home position and the position the chambers were rotated into. This was extracted according to

$$
\theta=\cos ^{-1}\left(\frac{\hat{R} * \hat{R}^{\prime}}{\|\vec{R}\|\left\|\vec{R}^{\prime}\right\|}\right)
$$

This defines the actual angle as defined by the home position after rotation into each 


\begin{tabular}{|l|l|}
\hline \multicolumn{2}{|c|}{ Vader $-90^{\circ}$} \\
\hline Point & Measured Angle \\
\hline VAH & -90.047 \\
\hline VBH & -90.042 \\
\hline VCH & -90.023 \\
\hline VDH & -90.035 \\
\hline VEH & -90.033 \\
\hline VFH & -90.020 \\
\hline VGH & -90.024 \\
\hline VHH & -90.029 \\
\hline
\end{tabular}

TABLE A.7: Shown is the extracted angle with respect to the home position.

\begin{tabular}{|l|l|}
\hline \multicolumn{2}{|c|}{ Yoda $-90^{\circ}$} \\
\hline Point & Measured Angle \\
\hline YAH & -90.248 \\
\hline YBH & -90.245 \\
\hline YCH & -90.238 \\
\hline YDH & -90.232 \\
\hline YEH & -90.229 \\
\hline YFH & -90.226 \\
\hline YGH & -90.192 \\
\hline YHH & -90.196 \\
\hline
\end{tabular}

TABLE A.8: Shown is the extracted angle with respect to the home position.

octant. Tables A.14 and A.13 define the repeatability after rotating the chambers to a given angle and then returning to home.

\section{Summary of Measurements}

Tables A.15 and A.16 give the standard deviation of the radial measurements for each tooling ball point, including all octants for each VDC package. For each package the $\mathrm{z}$ degree of freedom can be seen to be an important component of the repeatability and confirms the "tilt" of the rotator. Essentially the rotator itself, mostly likely the hub, was not situated perfectly along each axis and therefore rotation and radial motion do not move parallel along the axes. Instead there is a slight tilt so that there is motion in $\mathrm{Z}$ as well. 


\begin{tabular}{|l|l|}
\hline \multicolumn{2}{|c|}{ Vader $45^{\circ}$} \\
\hline Point & Measured Angle \\
\hline VAH & 45.018 \\
\hline VBH & 45.024 \\
\hline VCH & 45.005 \\
\hline VDH & 44.998 \\
\hline VEH & 44.992 \\
\hline VFH & 44.995 \\
\hline VGH & 44.966 \\
\hline VHH & 44.970 \\
\hline
\end{tabular}

TABLE A.9: Shown is the extracted angle with respect to the home position.

\begin{tabular}{|c|l|}
\hline \multicolumn{2}{|c|}{ Yoda $45^{\circ}$} \\
\hline Point & Measured Angle \\
\hline YAH & 44.970 \\
\hline YBH & 44.966 \\
\hline YCH & 44.945 \\
\hline YDH & 44.946 \\
\hline YEH & 44.938 \\
\hline YFH & 44.941 \\
\hline YGH & 44.926 \\
\hline YHH & 44.929 \\
\hline
\end{tabular}

TABLE A.10: Shown is the extracted angle with respect to the home position.

\begin{tabular}{|c|l|}
\hline \multicolumn{2}{|c|}{ Vader $90^{\circ}$} \\
\hline Point & Measured Angle \\
\hline VAH & 90.119 \\
\hline VBH & 90.134 \\
\hline VCH & 90.135 \\
\hline VDH & 90.109 \\
\hline VEH & 90.102 \\
\hline VFH & 90.123 \\
\hline VGH & 90.067 \\
\hline VHH & 90.070 \\
\hline
\end{tabular}

TABLE A.11: Shown is the extracted angle with respect to the home position. 


\begin{tabular}{|l|l|}
\hline \multicolumn{2}{|c|}{ Yoda $90^{\circ}$} \\
\hline Point & Measured Angle \\
\hline YAH & 89.964 \\
\hline YBH & 89.961 \\
\hline YCH & 89.931 \\
\hline YDH & 89.937 \\
\hline YEH & 89.924 \\
\hline YFH & 89.933 \\
\hline YGH & 89.928 \\
\hline YHH & 89.930 \\
\hline
\end{tabular}

TABLE A.12: Shown is the extracted angle with respect to the home position.

\begin{tabular}{|l|l|l|}
\hline \multicolumn{3}{|c|}{ Vader radial repeatability after rotation to the $-90^{\circ}$ position } \\
\hline Point & Angle Repeat & Radial Repeat \\
\hline VAH & 8.216 & 0.799 \\
\hline VBH & 8.021 & 0.736 \\
\hline VCH & 7.786 & 0.673 \\
\hline VDH & 2.375 & 1.131 \\
\hline VEH & 2.380 & 1.132 \\
\hline VFH & 7.792 & 0.672 \\
\hline VGH & 8.092 & 0.737 \\
\hline VHH & 8.328 & 0.806 \\
\hline
\end{tabular}

TABLE A.13: The repeatability of the measured angle and radius of the chambers after repeated rotation to the $-90^{\circ}$ position. Angles are shown in milli-degress and radial values are shown in millimeters.

\begin{tabular}{|l|l|l|}
\hline \multicolumn{3}{|c|}{ Yoda radial repeatability after rotation to the $-90^{\circ}$ position } \\
\hline Point & Angle Repeat & Radial Repeat \\
\hline YAH & 8.111 & 0.799 \\
\hline YBH & 6.898 & 0.736 \\
\hline YCH & 7.749 & 0.673 \\
\hline YDH & 11.358 & 1.131 \\
\hline YEH & 12.582 & 1.132 \\
\hline YFH & 6.890 & 0.672 \\
\hline YGH & 7.587 & 0.737 \\
\hline YHH & 7.761 & 0.807 \\
\hline
\end{tabular}

TABLE A.14: The repeatability of the measured angle and radius of the chambers after repeated rotation to the $-90^{\circ}$ position. Angles are shown in milli-degress and radial values are shown in millimeters. 


\begin{tabular}{|c|l|l|}
\hline \multicolumn{3}{|c|}{ Standard Deviation Radial Measurement in Theta: Vader } \\
\hline Point & Radial $\sigma(x, y, z)$ & Radial $\sigma(x, y)$ \\
\hline VAH & 1.577 & 3.473 \\
\hline VBH & 1.619 & 3.246 \\
\hline VCH & 1.705 & 3.482 \\
\hline VDH & 1.597 & 3.769 \\
\hline VEH & 1.596 & 3.789 \\
\hline VFH & 1.706 & 3.534 \\
\hline VGH & 1.643 & 3.540 \\
\hline VHH & 1.582 & 3.664 \\
\hline
\end{tabular}

TABLE A.15: The standard deviation of the radial measurement for each point in each position in theta. Extracted values are shown for the radius in both the (x,y) plane and the $(\mathrm{x}, \mathrm{y}, \mathrm{z})$ plane. Values in $\mathrm{mm}$.

\begin{tabular}{|l|l|l|}
\hline \multicolumn{3}{|c|}{ Standard Deviation Radial Measurement in Theta: Yoda } \\
\hline Point & Radial $\sigma(x, y, z)$ & Radial $\sigma(x, y)$ \\
\hline VAH & 1.678 & 2.468 \\
\hline VBH & 1.820 & 2.734 \\
\hline VCH & 1.559 & 2.475 \\
\hline VDH & 1.883 & 2.967 \\
\hline VEH & 1.579 & 2.479 \\
\hline VFH & 1.902 & 2.979 \\
\hline VGH & 1.841 & 2.506 \\
\hline VHH & 1.983 & 2.795 \\
\hline
\end{tabular}

TABLE A.16: The standard deviation of the radial measurement for each point in each position in theta. Extracted values are shown for the radius in both the (x,y) plane and the $(\mathrm{x}, \mathrm{y}, \mathrm{z})$ plane. Values in $\mathrm{mm}$ 


\begin{tabular}{|l|c|}
\hline \multicolumn{2}{|c|}{ Standard Deviation of Angular Measurements: Vader } \\
\hline Angle & $\sigma(\theta)$ \\
\hline-45 & 0.021 \\
\hline-90 & 0.009 \\
\hline 45 & 0.020 \\
\hline 90 & 0.027 \\
\hline
\end{tabular}

TABLE A.17: The standard deviation of the angles measured with respect to home of each measurement shown in Tables A.5-A.11.

\begin{tabular}{|l|c|}
\hline \multicolumn{2}{|c|}{ Standard Deviation of Angular Measurements: Yoda } \\
\hline Angle & $\sigma_{\theta}$ \\
\hline-45 & $\mathrm{~N} / \mathrm{A}$ \\
\hline-90 & 0.021 \\
\hline 45 & 0.016 \\
\hline 90 & 0.015 \\
\hline
\end{tabular}

TABLE A.18: The standard deviation of the angles measured with respect to home of each measurement shown in Tables A.6-A.12.

The standard deviation of the measured angle between each tooling point between home and each rotated position $\left(-90^{\circ},-45^{\circ}, 0^{\circ}, 45^{\circ}, 90^{\circ}\right)$ is shown in Tables A.17 and A.18. All units are in degrees and show that the angle between octants is within milli-degrees of the ideal separation.

The point of the measurements found in Tables A.19 and A.20 was to check for significant chamber sagging/flexing and bound the resolution of four measurements. For each rotational position the distance between pairs of tooling points is computed. As the chambers are static objects these points should not change within the resolution of the measurement. This gives an idea how well the position of each point was determined or the effective resolution considering any flexing or sagging effect that might be present. The distance between each point pair is given as well as the standard deviation between rotational positions. All units are in mm. From these tables we can determine that the point-to-point resolution of the survey measurements is on the few micron level. 


\begin{tabular}{|l|l|l|l|l|}
\hline \multicolumn{5}{|c|}{ Distance between static points: Vader } \\
\hline Point Pairs & BA & DF & CE & HG \\
\hline 0 & 412.490 & 839.544 & 839.825 & 412.379 \\
\hline-45 & 412.475 & 839.534 & 839.825 & 412.382 \\
\hline-90 & 412.488 & 839.540 & 839.818 & 412.383 \\
\hline 45 & 412.482 & 839.536 & 839.823 & 412.386 \\
\hline 90 & 412.477 & 839.545 & 839.819 & 412.387 \\
\hline \hline$\sigma$ & 0.007 & 0.005 & 0.003 & 0.003 \\
\hline
\end{tabular}

TABLE A.19: For each rotational position the distance between pairs of tooling ball locations (see Fig. A.1) is calculated. As these are static points the distance between them should not change. This gives a good estimate of the resolution with which the points were measured.

\begin{tabular}{|l|l|l|l|l|}
\hline \multicolumn{5}{|c|}{ Distance between static points: Yoda } \\
\hline Point Pairs & BA & DF & CE & HG \\
\hline 0 & 412.499 & 292.063 & 291.913 & 412.414 \\
\hline-45 & N/A & N/A & N/A & N/A \\
\hline-90 & 412.510 & 292.060 & 291.900 & 412.405 \\
\hline 45 & 412.501 & 292.065 & 291.917 & 412.4143 \\
\hline 90 & 412.497 & 292.063 & 291.903 & 412.415 \\
\hline \hline$\sigma$ & 0.006 & 0.002 & 0.008 & 0.005 \\
\hline
\end{tabular}

TABLE A.20: For each rotational position the distance between pairs of tooling ball locations (see Fig. A.1) is calculated. As these are static points the distance between them should not change. This gives a good estimate of the resolution with which the points were measured. 


\section{A.3 Background Correction Error Propagation}

The calculation of the individual systematic error contributions from the background correction to the physics asymmetry is done according to

$$
\sigma_{A}^{2}=\left|\frac{\partial A}{\partial b}\right|^{2} \sigma_{b}^{2}
$$

Applying this to Eq. 6.1 we get the following relations:

$$
\begin{aligned}
\left.\partial A\right|_{A_{m s r}} & =\frac{R \times d A_{\mathrm{msr}}}{P\left(1-f_{\text {total }}\right)} \\
\left.\partial A\right|_{A_{b l i n d}} & =\frac{R \times d A_{\mathrm{msr}}^{\text {blinding }}}{P\left(1-f_{\text {total }}\right)} \\
\left.\partial A\right|_{P} & =\frac{-R \times A_{\mathrm{msr}}}{\left(1-f_{\text {total }}\right)} \frac{d P}{P^{2}} \\
\left.\partial A\right|_{A_{i}} & =\frac{-R \times f_{i}}{\left(1-f_{\text {total }}\right)} d A_{i} \\
\left.\partial A\right|_{R} & =d R \times \frac{\left(\frac{A_{\mathrm{msr}}}{P}-\Sigma_{i} f_{i} A_{i}\right)}{\left(1-f_{\text {total }}\right)} \\
\left.\partial A\right|_{f_{i}} & =\epsilon_{i j k l}\left(\frac{A_{\mathrm{msr}}}{P}+\left(f_{j}+f_{k}+f_{l}\right) A_{i}-f_{j} A_{j}-f_{k} A_{k}-f_{l} A_{l}\right) \frac{R \times d f_{i}}{\left(1-f_{\text {total }}\right)}
\end{aligned}
$$

In the final equation the Levi-Civita operator is invoked to denote the permutation of the indices $1 \rightarrow 2 \rightarrow 3 \rightarrow 4$. With these calculations, the total systematic error contribution from backgrounds and experimental bias is given by

$$
\sigma_{A}=\sqrt{\left(\left.\partial A\right|_{A_{m s r}}\right)^{2}+\left(\left.\partial A\right|_{P}\right)^{2}+\left(\left.\partial A\right|_{A_{i}}\right)^{2}+\left(\left.\partial A\right|_{R}\right)^{2}+\left(\left.\partial A\right|_{f_{i}}\right)^{2}} .
$$




\section{A.4 Beam Modulation Improvements}

As was laid out in the Beam Modulation chapter, the system as a whole was a success in measurement of the helicity-correlated beam sensitivities. With every success however there is room for improvement, and there were a number of problems that were discovered in the system, that in hindsight, could have been fixed. In the interest of future modulation systems that might be built, I will address some of those issues and ways that they could have been avoided.

Fast-feedback This was a big issue in the analysis and running of the beam modu-

lation system. By leaving the fast-feedback system on during modulation another level of complexity was added into the analysis; because the fast-feedback system worked to counteract modulations in the beam position and angle the total response became a composite function with a phase out of response to the driving signal. This issue was missed in early testing of the system but in future iterations fast-feedback should be paused for the duration of the beam modulation period. This will lead to cleaner, more interpretable results.

Diagnostics and Tuning One of the pressing issues during the design, construction, and testing of the modulation system was a lack of manpower to provide the tools and do the diagnostic testing that was required to make the system the best it could be. A robust system would include online monitoring and diagnostic tools to give run-by-run results for things such as: beam monitor responses, detector responses, extracted sensitivities, and beam trajectory plots. The later of these would be enormously helpful as it would enable shift crew to monitor how separated the modulations were in phase space and call in experts to tune the modulation coils, given changes in the beam properties. This would ensure that the modulations - for example X-position and X-angle - were as pure posi- 
tion and angle as possible at all times leading to better extracted results. Too many times during the experimental running we didn't find problems until days after they appeared. A robust, real-time diagnostic system would ensure better response time to problems, and better results with each measurement. This of course requires an adequate amount of manpower to design and implement the tools. 